\title{
REAL-TIME MAGNETIC RESONANCE IMAGING
}

\author{
Dissertation \\ for the award of the degree \\ "Doctor of Philosophy" (Ph.D.) \\ Division of Mathematics and Natural Sciences \\ of the Georg-August-Universität Göttingen
}

submitted by

Shuo Zhang

from Shanghai, China

Göttingen 2009 
This work has been done at:

Biomedizinische NMR Forschungs GmbH

am Max-Planck-Institute für biophysikalische Chemie

Members of the Thesis Committee:

Prof. Dr. Jens Frahm (Reviewer) Biomedizinische NMR Forschungs GmbH am Max-Planck-Institut für biophysikalische Chemie

Prof. Dr. Ulrich Parlitz (Reviewer) III Physikalisches Institut Georg-August-Universität Göttingen

Prof. Dr. Annette Zippelius Institut für Theoretische Physik Georg-August-Universität Göttingen

Date of the oral examination: 
I hereby declare that this dissertation "Real-time Magnetic Resonance Imaging" has been written independently and with no other sources and aids than quoted.

Göttingen, September $18^{\text {th }}, 2009$

Shuo Zhang 


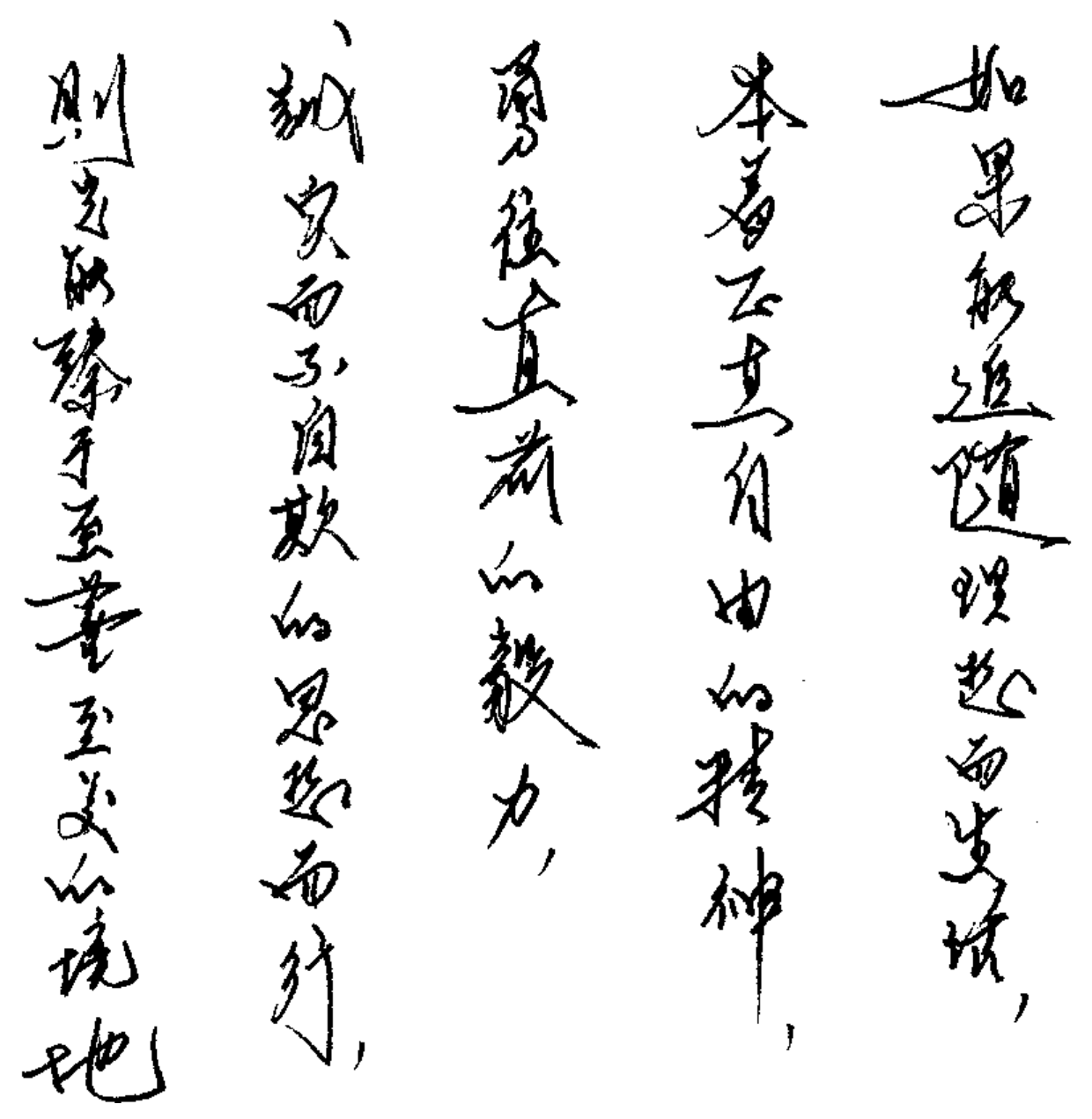


The Chinese text on the other side of this page is said to be a quote from Madame Marie Curie (18671934). Unfortunately, its original context has been lost despite my best efforts in trying to find it. Nevertheless, the Chinese version is famous for its very appropriate translation and at the same time the utmost beauty of utilizing the language - it not only describes a golden rule in science but also delivers a philosophical theme for the life of humans. Under such circumstances, I could only try to give a very rough translation in the hope of not losing the accuracy and flavor of the Chinese version:

If one could live up to the ideals,

in the light of free spirit and conscientiousness,

full of perseverance and courage,

with honesty and sincerity, then

we shall consummate the perfection in every respect.

The calligraphy of this Chinese text is written by my father Professor Kangle Zhang. It is read from upper right to lower left. 


\section{CONTENTS}

CHAPTER 2. Fundamental MR Physics and Techniques

$\begin{array}{lll}2.1 & \text { NMR Phenomenon and Signal Generation } & 7\end{array}$

2.2 Signal Localization and Image Production 11

2.2.1 Spatial Encoding and Gradient Control 11

2.2.2 k-Space Formalism and Fourier Imaging 14

$\begin{array}{ll}\text { 2.2.3 Sampling Requirements } & 16\end{array}$

$\begin{array}{lll}2.3 & \text { Sequence Type and Contrast Mechanism } & 17\end{array}$

2.4 MRI System 22

2.5 Summary 24

CHAPTER 3. Development of Real-time MRI: Physical Basis 25

3.1 Signal Acquisition with the FLASH Technique 26

3.1.1 Spoiled Steady-State Free Precession (SSFP) 26

$\begin{array}{lll}3.1 .2 & \text { Refocused SSFP } & 27\end{array}$

$\begin{array}{lll}\text { 3.1.3 Balanced SSFP } & 28\end{array}$

3.2 Comparison of Available Acquisition Techniques 29

3.2.1 Brief Review 29

3.2.2 Experimental Comparison 33

3.3 Further Improvements for Ultra-fast MRI 39

3.3.1 RF Pulse Duration, Slice Profile and Partial Volume Effect 39

3.3.2 Receiver Bandwidth, SNR and Chemical Shift Artifact 40

3.3.3 Echo Time and Susceptibility Artifacts 41

3.3.4 Repetition Time, Flip Angle and Contrast 42

3.3.5 Spike Noise Reduction 43

$\begin{array}{lll}3.4 & \text { Summary } & 46\end{array}$ 
CHAPTER 4. Development of Real-time MRI: $\boldsymbol{k}$-Space Encoding

4.1 Spatial Encoding with Radial Trajectories 48

4.1.1 Undersampling beyond the Nyquist Limit 49

4.1.2 Free Zooming without Aliasing 50

4.1.3 Intrinsic Robustness against Motion $\quad 50$

4.2 Comparison of Available Encoding Schemes $\quad 51$

4.2.1 Brief Review $\quad 51$

4.2.2 Experimental Comparison

4.3 Specific Optimization for Time-resolved MRI 52

4.3.1 View Number, Rotational Angle and Gradient Polarity 62

4.3.2 Trajectory Correction $\quad 65$

$\begin{array}{lll}\text { 4.3.3 View Reordering } & 68\end{array}$

$\begin{array}{lll}4.4 & \text { Summary } & 71\end{array}$

CHAPTER 5. Development of Real-time MRI: Image Reconstruction 73

$\begin{array}{lll}5.1 & \text { Reconstruction with Gridding } & 73\end{array}$

5.2 Update with Sliding Window and View Sharing 77

5.2.1 Sliding Window and MR Fluoroscopy 77

5.2.2 Cartesian Keyhole Imaging $\quad 79$

5.2.3 Interleaved Radial Sliding Window $\quad 80$

5.3 Comparison of Available Reconstruction Strategies 81

5.3.1 Brief Review

5.3.2 Experimental Comparison $\quad 84$

$\begin{array}{lll}5.4 & \text { Summary } & 93\end{array}$

CHAPTER 6. Realization of Real-time MRI on 3T Human MRI System 95

6.1 Radial FLASH - Pulse Sequence Design 95

6.1.1 Pulse Sequence Design 95

$\begin{array}{lll}6.1 .2 & \text { Image Contrasts } & 97\end{array}$

6.2 Radial FLASH - Image Quality 102

$\begin{array}{lll}\text { 6.2.1 Spatial Resolution } & 103\end{array}$

6.2.2 Temporal Resolution 105

$\begin{array}{llr}6.3 & \text { Receiver Channel Compression } & 108\end{array}$ 
7.1 Ocular Movement

7.2 Temporomandibular Joint

7.3 Musculoskeletal System

7.4 Speech Production

7.5 Swallowing Process

7.6 Cardiovascular Motion

\section{Appendix}

A - Workflow Chart and Hardware Topology of Real-time MRI

B - Quantum Mechanical Description of the NMR Phenomenon

Bibliography 


\section{Chapter 1}

\section{Introduction}

MAGNETIC RESONANCE IMAGING (MRI) is one of the most important medical imaging modalities. It is based upon the phenomenon of nuclear magnetic resonance (NMR), and uses strong magnetic fields and radiofrequency (RF) radiation to translate the distribution of hydrogen nuclei (protons) in body tissue into tomographic images of anatomical structures. The principle of MRI was first introduced in the 1970s. Today there are almost 100 million MRI examinations being performed worldwide each year, in both clinical routine and biomedical research.

A general advantage of MRI over radiographic imaging techniques such as computed tomography is its non-invasiveness, which is due to the absence of ionizing radiation. It provides images from cross-sections or volumes along arbitrary orientations without problems related to finite penetration depth and internal reflection, as for example known from ultrasound techniques. In addition, the images have excellent soft tissue contrast, superior to that of any other imaging techniques, which facilitates diagnosis and monitoring of disease progression and treatment in various organs including brain, heart, joints, and breast. With access to a large variety of contrast mechanisms based on magnetic relaxation times, susceptibility differences, motions, chemical shift, and magnetization transfer properties, MRI is not restricted to morphological descriptions of the anatomy, but also serves as a powerful tool in interventional, functional, metabolic and quantitative imaging studies of diverse tissue properties.

On the other hand, and despite considerable progress in the technical development, there is still a lack of established MRI methods for continuous imaging of moving objects in real time. In fact, experimental trials in research-oriented environments and eventual applications in a clinical setting are of a very preliminary character. In this thesis, a generic solution for real-time MRI (RT-MRI) with excellent image quality, spatial accuracy and temporal fidelity is presented [1]. 


\section{History}

The history of MRI can be traced back to about half of a century ago when the NMR phenomenon was discovered independently by Edward Purcell and Felix Bloch in 1946 [2, 3], for which both were awarded the Nobel Prize in Physics six years later. In 1950, Erwin Hahn demonstrated that the NMR effect can also be observed after the application of brief RF pulses [4]. The concept of this pulsed NMR experiment was later exploited for all MRI techniques. In the next twenty years, NMR was exclusively used for analytical spectroscopic purposes, mainly to study molecular structures and functions of chemical systems. In 1971 Raymond Damadian observed that the nuclear magnetic relaxation times of water protons in healthy and cancerous tissue differ, motivating scientists to consider NMR for disease detection [5].

In 1973 Paul C. Lauterbur for the first time described a way to spatially resolve NMR signals and to reconstruct a two-dimensional image from multiple encoding steps [6]. By superimposing magnetic gradient fields to the main field during signal detection, the resonance frequency was made position-dependent, permitting reconstruction of the spatial distribution of resonant nuclei by spectral analysis of the detected signal. For this breakthrough Lauterbur was awarded the Nobel Prize in Physiology or Medicine in 2003. Based on the gradient encoding approach, in 1975 Richard R. Ernst, who was awarded the Nobel Prize in Chemistry in 1991, proposed a modified version termed Fourier imaging [7], For spatial encoding, the use of a rotating frequency-encoding gradient was replaced by the combination of a variable phase-encoding gradient and a fixed frequency-encoding gradient. Accordingly, the image reconstruction by projection reconstruction from the Lauterbur experiment was replaced by a two-dimensional Fourier transformation. This concept has since then formed the basis of the vast majority of MRI techniques.

In Fourier imaging the signal acquisition is conveniently described using the so-called $k$ space formalism [8, 9]. In this fashion, gradient switching corresponds to playing out a trajectory in the spatial frequency domain, or $k$-space, with the net gradient direction and magnitude corresponding to $k$-space speed. Signal registration along the $k$-space trajectory is equivalent to sampling the Fourier transform of the spatial signal density. Accordingly, the desired image is reconstructed by an inverse Fourier transformation of the acquired data. In 1976, the first human MR image of a finger was acquired [10], rapidly followed by reports of other body regions [11-14]. Subsequent technical advances in magnet and gradient hardware made it possible to design MRI scanners for clinical trials during the early years of the 1980s. 


\section{Technical Considerations}

As MRI became one of the common methods for diagnostic imaging, its drawbacks were also recognized. Apart from the fact that patients with a cardiac pacemaker or other metallic implants have to be excluded, this mainly applies to the relatively long measuring times. For conventional MRI, the acquisition of a spatially encoded NMR signal has to be repeated many times to cover the $k$-space with sufficient density and extent (typically 128 to 256 times), in order to yield a high-resolution image with diagnostic value. This is commonly accomplished in a rectilinear or Cartesian sampling grid. Most importantly, however, a certain time has to be awaited between each acquisition (typically 500 to $2000 \mathrm{~ms}$ ) to ensure sufficient signal recovery. This limit becomes a substantial problem for MRI, because long imaging times cause motion problems for abdominal or cardiac examinations as well as for less cooperative patients, and generally do not allow for dynamic studies with a temporal resolution of seconds that are typical for many physiological processes.

Over recent decades, a great number of new techniques and strategies have emerged that attempt to overcome this problem. The first leap was the invention of fast imaging pulse sequences, which are able to generate and acquire multiple echo signals continuously or compactly after excitation. These techniques include echo-planar imaging (EPI) [15], fast low-angle shot (FLASH) imaging [16], and rapid acquisition with relaxation enhancement (RARE) [17] techniques. In all these cases, the measuring time could be reduced considerably albeit with different image contrasts and sensitivities to other undesired conditions such as susceptibility artifacts (as in EPI) or high RF power (as in RARE). Though generally included and widely used in MRI, these imaging methods alone do not offer a general solution to achieve RT-MRI.

A variety of other strategies were therefore suggested to further speed up MRI with Cartesian encoding of the $k$-space. These include reconstructions from acquisitions with more efficient data sampling schemes that cover only a part of $k$-space [18]. Examples are the "sliding window" technique [19], which updates the $k$-space in sequential segments, and the "keyhole" technique [20,21], which updates only the low spatial frequency components. More recently, the different spatial sensitivities from multiple receiver coils were exploited for parallel imaging (or parallel acquisition techniques, PAT). This complementary information allows for image reconstruction from a sparsely sampled (undersampled) $k$-space [22-25]. In this way, considerable scan time reduction was achieved by reducing the number of excitation steps without a loss of spatial resolution or the introduction of aliasing artifacts. Nevertheless, 
these imaging methods cannot overcome inconsistent phase errors due to the object motion and do not provide sequential data sets that allow for a smooth update when imaging a dynamic process.

\section{Purpose}

Therefore, the primary aims of this thesis are:

- a comprehensive analysis of the physical requirements for RT-MRI,

- the development of a generic solution for RT-MRI offering high flexibility to a broad range of potential applications,

- the implementation of the proposed RT-MRI method on a 3 Tesla human whole-body MRI system, and

- an experimental validation using proof-of-principle studies of healthy human subjects.

The developed method is expected to monitor dynamic processes in real time and the potential applications should allow for the monitoring of active body movements such as cardiovascular motions as well as of external objects such as the delivery of a contrast agent or the administration of a biopsy needle or catheter during surgery. In this context, RT-MRI refers to the acquisition, reconstruction, and display of magnetic resonance images without any noticeable delay. This definition deliberately ignores any retrospective or offline reconstruction strategies with more advanced but slower mathematical algorithms or with specifically dedicated hardware that is normally unavailable.

In order to accomplish these goals, my thesis comprises both theoretical analyses and practical implementations. As the central result, a RT-MRI technique is proposed that combines the FLASH acquisition principle with a radial encoding scheme, a sliding window reconstruction algorithm, and a $k$-space view sharing technique. The ideas are implemented on a commercial MRI system without the need for any hardware modification. Multiple applications indicate substantial scientific and clinical potential. 


\section{Outline}

A brief introduction of the fundamental MR physics and techniques is given in Chapter 2 comprising a description of the signals that arise from the NMR phenomenon, the $k$-space encoding and image formation using traditional Fourier imaging, the image contrast based on basic pulse sequences, and the MRI hardware equipment used for the experiments.

As the first step toward RT-MRI, Chapter 3 compares various rapid MRI acquisition techniques with respect to their physical principles. The advantages of the FLASH technique are experimentally demonstrated. The results show a high flexibility between acquisition time and image resolution. Moreover, the absence of image artifacts and the low RF energy deposition make it the best choice for rapid and continuous imaging [26].

As the second module in the development of RT-MRI, Chapter 4 compares different $k$ space sampling techniques for spatially encoding the acquired MR signal. In particular, the radial encoding scheme, as originally proposed by Lauterbur, is favored against the conventional Cartesian scheme. It has long been experimentally hampered by technical inaccuracies of the early MRI systems, which inevitably led to the overwhelming predominance of Cartesian sampling schemes. However, radial encoding is recently regaining popularity, because, unlike Cartesian encoding, its lack of a phase-encoding gradient renders it tolerant to data undersampling, and in addition, its inherent oversampling in the $k$-space center, which contributes to gross image contrast, makes radial MRI insensitive to object motion during data acquisition. These two prominent features, namely the benign undersampling behavior [27, 28] and motion robustness [29], represent particular advantages for accelerated acquisitions and dynamic imaging.

As the third major component of RT-MRI, Chapter 5 covers the concepts for image reconstruction. It describes the gridding technique for radially encoded $k$-space data [30], which is followed by a conventional 2D inverse Fourier transform. In addition, view sharing of successive acquisitions combined with a sliding window algorithm is applied to decouple data acquisition and image reconstruction, and to realize a smooth update of the dynamic process together with an interleaved radial encoding scheme.

Chapter 6 focuses on the practical realization of the proposed RT-MRI method in a real environment based on the previous development. Implementation is achieved on a human whole-body MRI system with a magnetic field strength of 3 Tesla. The strategies for pulse sequence design are presented; the image contrast and quality, as well as achievable spatial and temporal resolution are determined. 
In Chapter 7 the proposed RT-MRI method is experimentally validated in human subjects covering a wide range of potential applications. Examples include RT-MRI of the movements of the eyes and joints, the speech production, the swallowing precess, and the cardiovascular motion without electrocardiography (ECG) synchronization or breath hold [1].

Finally, Chapter 8 summarizes the main achievements of this thesis and presents an outlook of the prospective work in the future. 


\section{Chapter 2}

\section{Fundamental MR Physics and Techniques}

This chapter gives a brief outline of the basic MRI principles, including nuclear physics, signal processing and tomographic imaging, as well as hardware equipment, as far as necessary for this thesis. For more comprehensive details see [31-33].

\subsection{NMR Phenomenon and Signal Generation}

The physical principle of nuclear magnetic resonance (NMR) can be summarized as the observation of high frequency signals emitted by atomic nuclei with a magnetic dipolar moment when placed in a strong magnetic field and excited by a short radiofrequency (RF) pulse with proper energy. It can be categorized into three sequential steps: (1) polarization, during which the macroscopic magnetization is formed by the equilibrium of nuclear spins aligned in the applied static magnetic field; (2) resonance, during which the perturbation of this alignment of the nuclear spins occurs when absorbing energy from an electromagnetic pulse at a specific frequency; (3) relaxation, during which the nuclear spins in a nonequilibrium state return to the equilibrium by releasing the absorbed energy. Before proceeding to the details, the fundamental property of spins that enables NMR and MRI is introduced, and then the Bloch equations are used to describe the dynamics of macroscopic nuclear magnetization in the above steps as a straightforward model, while a quantum mechanical description on a microscopic scale, is not given here but instead included in Appendix B.

\section{Spin}

Spin is the quantum mechanical description of the intrinsic quantized angular momentum $(L)$ observed in subatomic particles such as protons, electrons, neutrons or even photons, and also the entire nucleus. It is distinct from the orbital angular momentum derived from modeling 
the dynamics in the classical physics description. Because all elements consist of nuclei, which comprise protons and neutrons, and each of these individual unpaired elementary particles have a spin of $1 / 2$, all atomic nuclei with an odd number of protons or neutrons possess a net spin as well. Such nuclei are relevant for NMR. In particular, hydrogen nuclei (commonly referred to as protons) are most suitable for MRI because their natural ubiquity in water and fat, of which the human body consists, allow medically meaningful images to be obtained within reasonable measuring times.

As a net magnetic moment $(\mu)$ is produced parallel to the rotation axis by the net ionic charge distribution due to the unpaired nucleon, such a nucleus behaves like a magnetic dipole, which can be considered as a miniature bar magnet. As the orientation of such dipoles is random without external magnetic field, the magnetization of the nuclei cancels out on the average and the total magnetic moment is zero, in which case there is no net magnetization.

\section{Polarization and Magnetization}

When placed in a magnetic field $B_{0}$, these magnetic moments will polarize and, for hydrogen, these protons will line up either parallel (spin-up) or anti-parallel (spin-down) relative to the applied magnetic field. This alignment is not exact because as protons spin, they wobble, much as a spinning top. Such wobbling of the axis of rotation about the magnetic field is called precession and the resulting magnetization vector, representing the magnetic dipole moments of the aligned protons, lies along the axis of precession.

Because anti-parallel protons require a higher energy state, more protons align in the parallel direction. Thus, a macroscopic magnetization arises from the population difference in the direction of the external magnetic field (z-axis) within a volume of tissue when looking at an ensemble of spins. This magnetization $(M)$, i.e. the sum of the individual magnetic moments $\sum \mu$, is proportional to the angular momentum $(L)$ through the gyromagnetic ratio $(\gamma)$, which is constant for a given type of atom (for hydrogen, $\gamma=42.58 \mathrm{MHz} / \mathrm{T}$ ):

$$
M=\gamma L
$$

At the same time it also experiences a torque, or twisting force, in the magnetic field according to the classical electromagnetism laws, given by the vector cross-product $M \times B_{0}$. And because the torque of a system is equal to the time rate of change of its angular momentum $d L / d t$, as a result, the motion of the magnetization over time $(d t)$ in the constant magnetic field $B_{0}$ could be described by the vector equation:

$$
\frac{d M}{d t}=\gamma M \times B_{0}
$$


The precession frequency, called the Larmor frequency $\omega_{0}$, is proportional to the magnetic field strength:

$$
\omega_{0}=\gamma B_{0}
$$

This equation indicates that higher field strength results in higher frequency and that each type of nucleus precesses at a unique frequency in a given magnetic field. For example, when exposed to a magnetic field strength of $B_{0}=2.89$ Tesla $(\mathrm{T})$, which is used in this thesis, it corresponds to an angular frequency of $\omega_{0}=7.74 \cdot 10^{8} \mathrm{rad} / \mathrm{s}$, or respectively, $f_{0}=\omega_{0} / 2 \pi=$ 123.2 $\mathrm{MHz}$.

\section{Excitation and Resonance}

Because the net magnetization vector is in the same direction as the applied external magnetic field, a displacement is necessary in order to detect signals from it. Therefore, an additional transient oscillating magnetic field with the same Larmor frequency is applied in a plane perpendicular to the net magnetization. Since this frequency belongs to the spectrum used for radio transmission, this electromagnetic field is called a $R F$ pulse.

Absorption of RF energy by the hydrogen protons of the same frequency as that of their precession (known as the phenomenon of resonance) causes the magnetization $M$ to rotate away from its thermal equilibrium position and results in transitions in spin states from lower to higher energy (excitation). The angle of rotation $(\alpha)$, called RF flip angle $(F A)$, is controlled by the magnitude of the applied oscillating field $\left(B_{1}\right)$ and its length of time $\left(\tau_{p}\right)$ :

$$
\alpha=\gamma \int_{t=0}^{\tau_{p}} B_{1} d t
$$

In a frame that is rotating about the direction of the main static magnetic field $B_{0}$ at $\omega_{0}$, the magnetization appears to precess only about $B_{1}$ and the above effect is more easily seen as the magnetization $M$ is tipped away from the longitudinal direction, i.e. z-axis, into the transverse plane, i.e. the x-y plane, with a flip angle $\alpha$.

At the same time, another important effect of the RF pulse is that it causes the precessions of the protons to be synchronized so that they rotate in phase. Right after switching off the RF pulse, the spin system will return to its equilibrium state (relaxation) and the transverse magnetization $M_{x y}$ will decay exponentially. 


\section{Relaxation and Free Induction Decay}

The whole relaxation phenomenon occurs through a twofold process and is measured as two time constants, T1 and T2. T1 recovery, the spin-lattice or longitudinal relaxation time, describes the return of the longitudinal component of the magnetization $\left(M_{z}\right)$ to the thermal equilibrium, i.e. its original alignment with the static magnetic field, and represents the time it takes for $63 \%$ of the protons to return to the lower energy state. Energy loss results from magnetic interactions between protons that transfer energy to surrounding molecules. The mathematical description of this process is given by:

$$
\frac{d M_{z}}{d t}=-\left(M_{z}-M_{0}\right) / T 1
$$

with the solution of its behavior as a function of the time $t$ :

$$
M_{z}=M_{0}\left(1-e^{-\frac{t}{T 1}}\right)
$$

T2 decay, the spin-spin or transverse relaxation time, represents the time required for the transverse component of the magnetization $\left(M_{x y}\right)$ to decrease by $63 \%$ as a result of precessional dephasing, i.e. loss of phase coherence. Energy loss results from the interaction between protons with neighboring protons and magnetic field disturbances. The value of T2 for a given material is typically much less than $\mathrm{T} 1$, and the analytical description of this process is given by:

$$
\frac{d M_{x y}}{d t}=-M_{x y} / T 2
$$

with the solution of its behavior over time $t$ :

$$
M_{x y}=M_{0} e^{-\frac{t}{T 2}}
$$

Both $\mathrm{T} 1$ and $\mathrm{T} 2$ are specific to the type of tissue, and therefore are used to characterize the type and physiologic state of the tissue as the basis for image contrast in MRI (see Chapter 2.3). The value of $\mathrm{T} 1$ for hydrogen protons in living tissue ranges from 400 to 1500 milliseconds (ms), and the value of T2 ranges from 50 to $150 \mathrm{~ms}$.

Noticeably, in real systems, minor differences in chemical environment which lead to a distribution of resonance frequencies may also lead to dephasing and signal loss. Although such de-coherence due to magnetic field heterogeneity (e.g. main magnetic field inhomogeneities, magnetic susceptibility transitions, contrast agent, etc.) is not a true "relaxation" process, for many NMR experiments this "relaxation" dominates. Compared to the ideal T2 relaxation, which refers to the non-reversible dephasing caused by spin-spin interaction, such signal deviation can be recovered, and it is consistent over time for molecules that are not moving. The corresponding transverse relaxation time constant is termed $\mathrm{T} 2 *$, which is 
usually much smaller than T2. The relation between them is:

$$
\frac{1}{T 2^{*}}=\frac{1}{T 2}+\frac{1}{T_{\text {inhom }}}=\frac{1}{T 2}+\gamma \Delta B_{0}
$$

where $\Delta B_{0}$ is the difference in strength of the locally varying field.

In summary, by taking account of all magnetic fields and relaxation effects (and neglecting field inhomogeneity, flow, and diffusion processes), the torque motion of the magnetization vector in the laboratory (stationary) frame of reference is described by the Bloch equations, which were introduced by Felix Bloch in 1946 as phenomenological equations for $M=$

$$
\begin{aligned}
\left(M_{x}, M_{y}, M_{z}\right) & \\
\frac{d M_{x}}{d t} & =\gamma M \times B_{x}-\frac{M_{x}}{T 2} \\
\frac{d M_{y}}{d t} & =\gamma M \times B_{y}-\frac{M_{y}}{T 2} \\
\frac{d M_{z}}{d t} & =\gamma M \times B_{z}-\frac{M_{z}-M_{0}}{T 1}
\end{aligned}
$$

The magnetic field $B$ comprises both static and oscillating components.

As the spin system relaxes and returns to its equilibrium state, the precessing magnetization oscillates at the Larmor frequency. Since only the transverse component of the magnetization can induce current in a coil, which is placed perpendicular to the $B_{1}$ direction, the received signal will decay rapidly. This is known as the phenomenon of free induction decay (FID).

\subsection{Signal Localization and Image Production}

In NMR, all protons in the examined tissue are excited at once by the RF pulse. Therefore, the overall signal, i.e. the sum of signals from all protons in that volume of the tissue, is measured. Through a Fourier transformation (FT) of the FID signal, the multiple resonance frequencies due to different chemical species can be represented in the frequency domain with distinguishable peaks in a spectrum. This can be used to study the chemical structure of the tissue and is referred to as NMR spectroscopy. To be able to identify the source of the resonance signal and to produce images of the tissue, specific manipulation of the magnetization is exploited in MRI. The physical principle of this procedure can be summarized as the visualization of the NMR image by (1) controlling the imaging pulse 
sequence for spatial encoding of the signal in the $k$-space via modifying the resonance condition with variable magnetic fields (gradients) and (2) using a Fourier transformation for image reconstruction.

\subsubsection{Spatial Encoding and Gradient Control}

The received MR signal $(S)$ represents the transversal component of the assembly of all spin signals from the excited volume $(V)$ :

$$
S(t)=\int_{V} M_{x y}(t) \cdot d V
$$

where the precession of the magnetization vector in transverse plane $M_{x y}$ at time $t$ after excitation, based on the Bloch equations but neglecting all relaxation effects for simplicity till further notice, is:

$$
M_{x y}(t)=M_{x y}(0) e^{-i \gamma B_{0} t}
$$

in which $M_{x y}$ refers to a complex quantity $M_{x y}=M_{x}+i M_{y}$.

In order to spatially encode the signal, small linear magnetic field gradients $(G)$ are applied that lead to spatially diverse precession frequencies in a volume. By superimposing such a linear gradient to the static magnetic field $B_{0}$, the following spatially-dependent magnetic field is obtained:

$$
B(r)=B_{0}+r G_{r}
$$

where $G_{r}$ refers to the gradient strength in the r-direction. Because the Larmor frequency $\omega_{0}$ is proportional to the field strength, it also becomes position dependent:

$$
\omega(r)=\gamma\left(B_{0}+G_{r} r\right)=\omega_{0}+\gamma G_{r} r
$$

In other words, the gradient fields allow for linking the Larmor frequency to the spatial location inside the magnet. Thus, spins at different locations can be differentiated by examining their resonance frequencies. The basic concepts of using gradients for spatial encoding in this manner, i.e. gradient encoding, are described below.

\section{Slice Selection Gradient}

Just considering the above condition, by imposing a magnetic field gradient $G_{z}$ in the z-axis direction on the main field $B_{0}$, the resonance frequency of the tissue becomes linearly dependent on the position along the z-axis. Due to the quantization of the energy levels, 
protons can only be excited if the frequency of the RF pulse coincides with the resonance frequency, $\omega(z)$ in this case. Therefore, the position of the protons to be imaged can be selected by adjusting the gradient field and the RF pulse:

$$
z=\left(\frac{\omega}{\gamma}-B_{0}\right) / G_{z}
$$

In practice, an RF pulse with a narrow bandwidth of $\Delta \omega=\gamma G_{z} \Delta z$ selectively excites a thin slice of thickness $\Delta z$ (typically several millimeters) and reduces the localization problem from $3 \mathrm{D}$ to $2 \mathrm{D}$. This concept is called slice excitation or slice selection. The selected slice is further encoded by field gradients in the $\mathrm{x}$ and $\mathrm{y}$ directions. To serve as a base for encoding techniques, only the traditional approach, i.e. encoding in a Cartesian grid, is introduced in the following. A detailed description and comparison with other advanced approaches, e.g. encoding in a radial manner, is given in Chapter 4.

\section{Frequency Encoding Gradient}

A frequency-encoding gradient (also called read or readout gradient, $G_{x}$ ), is applied along xaxis direction after slice-selection excitation during the acquisition or readout time in one measurement. This results in spatially dependent resonance frequencies along the $\mathrm{X}$-axis:

$$
\omega(x)=\gamma\left(B_{0}+G_{x} x\right)
$$

which yields spatially dependent magnetization:

$$
M_{x y}(t)=M_{x y}(0) e^{-i \gamma \int\left[B_{0}+G_{x}(t) x\right] d t}=M_{x y}(0) e^{-i \gamma \int\left[B_{x}(t)\right] d t}
$$

The received signal is a sum of harmonic functions with different frequencies and amplitudes. By performing a FT, the distribution of frequencies describes the one-dimensional spatial distribution of the spins in the sample, and the amplitude of each peak describes the amount of the magnetization (proton density or another property of the tissue) at each location (voxel).

\section{Phase Encoding Gradient}

For a one-dimensional experiment, the encoding with a frequency-encoding gradient alone would be sufficient. However, to create two- or three-dimensional images, another gradient is needed to encode the information in the second or third dimension - the phase-encoding gradient. It is applied after the excitation pulse but before the acquisition in a direction perpendicular to the read direction, i.e. along the y-axis direction. The effect of the phase gradient is the same as that of the read gradient, however, it is applied only for a short time, during which the precessing frequencies are affected and spatially altered. After the gradient 
is turned off, the spins return to the original frequency but with different phases $\varphi_{y}$, depending on their location along the y-axis, which have been acquired when the phase gradient is on:

$$
\varphi_{y}=\gamma G_{y} y t
$$

where $t$ is the total amount of time the phase gradient has been applied. The magnetization along the $y$-axis is then given by:

$$
M_{x y}(t)=M_{x y}(0) e^{-i \gamma \int\left[B_{0}+G_{y}(t)\right] d t}=M_{x y}(0) e^{-i \gamma \int\left[B_{y}(t)\right] d t}=M_{x y}(0) e^{-i \varphi_{y}(t) d t}
$$

This phase modulation is not affected by gradients applied in perpendicular directions during the rest of the measurement, including the read gradient. Furthermore, one data acquisition period does not supply enough information to create a 2D image because only one phase shift has been induced. Thus, the measurement must be repeated $N_{y}$ times, where $N_{y}$ is the desired number of points in the y direction. With each repetition, either the phase gradient strength $\left(G_{y}\right)$ or its duration $(t)$ is changed, giving the spins a different phase shift for each acquisition. Once the data is complete, a 2D inverse Fourier transformation (iFT) is performed to yield a two-dimensional image of the spin density.

\subsection{2 $k$-Space Formalism and Fourier Imaging}

After introducing the three magnetic field gradients, it is necessary to reconsider the received MR signal. For simplicity, a volume in two dimensions, i.e. a slice after RF selection, is described. First of all, this complex signal $S_{r}$, arising from the induced voltage in the receive coils by the transverse magnetization precessing at a time $t$ from an arbitrary source location $r$ is given by:

$$
S_{r}(t)=e^{-i \int \omega_{r}(t) d t}
$$

By considering the application of a generalized gradient, the resonance frequency is a function of not only time but also position:

$$
S_{r}(t)=e^{-i \gamma \int G_{r}(t) r d t}
$$

In general, the magnetization is described as the summation, i.e. integration of the continuous distribution of the spin density over space $\rho(r) d r$, which yields:

$$
S_{r}(t)=\int \rho(r) e^{-i \gamma \int G_{r}(t) r d t} d r
$$

or the same by considering the local precessing phase (Eq 2.7) that accumulated while the gradients are switched on: 


$$
S_{r}(t)=\int \rho(r) e^{-i \gamma \int \varphi_{r}(t) d t} d r
$$

Because the gradient $G(t)$ is a known function, by defining a reciprocal spatial term $k$ as:

$$
k(t)=\frac{\gamma}{2 \pi} \int G(t) d t
$$

where, for the usual case of a linear (i.e. time-independent) gradient:

$$
k(t)=\frac{\gamma}{2 \pi} G t
$$

the signal can be re-written as:

$$
S(k)=\int \rho(r) e^{-i 2 \pi k r} d r
$$

which is recognized as Fourier transform of the proton density $\rho(r)$ at the position $k(t)$ in the Fourier domain. Inversely:

$$
\rho(r)=\int S(k) e^{i 2 \pi k r} d k
$$

which reveals that the spatial information can be obtained from the Fourier domain data by iFT. This Fourier domain is commonly referred to as $k$-space in MRI, which offers a unique means to illustrate how the spatial frequencies are encoded. Eq 2.12 also shows that the received signal is complex. Thus, the image to be reconstructed has complex entries as well, but usually it is the magnitude values that are presented for viewing.

It should be noted that the relaxation is so far ignored for simplicity. Thus, an accurate description of the received signal needs an extension of Eq 2.12 by including respective relaxation terms into the integral. And the received signal corresponds actually to the Fourier transform of modulated spin density. By controlling the timing of the signal acquisition via imaging pulse sequences, different parameters can be emphasized to provide parameter weighted images with different contrast appearances (see later).

With a proper gradient control, a particular trajectory (path) is steered to cover the $k$ space. Firstly, the $k$-space location (Eq 2.11) in one measurement can be understood as the momentum of a gradient - for a linear gradient - the gradient strength multiplied by its duration. Such momentum is important when examining refocusing and defocusing of magnetization using gradients. Together with several important advanced imaging pulse sequences for accelerated MR imaging, they are presented and compared in Chapter 3. Secondly, as can be seen from Fig 2.1 where the intensity in the $k$-space is displayed in logarithmic scale, rather than a monotonously homogeneous domain, the centeral $k$-space area with high intensity values corresponds to the low frequency component of the signal that contributes to the image gross contrast, while the peripheral area with low intensity values is filled up with high frequency components that contributes to the image details. Therefore, it is necessary to switch the gradient in such a way that the spatial frequency signal is collected not 
only sufficiently along the trajectory to cover the $k$-space, but also efficiently for image reconstruction to represent the sampled object. Such encoding schemes are described in Chapter 4.

Furthermore, through any linear combination of gradients, an imaging slice can be selected in an arbitrary orientation. As both two- and three-dimensional imaging methods are common in MRI, the $k$-space has also the same dimensional order as the image to be acquired. This dissertation entirely focuses on 2D imaging for real-time application.

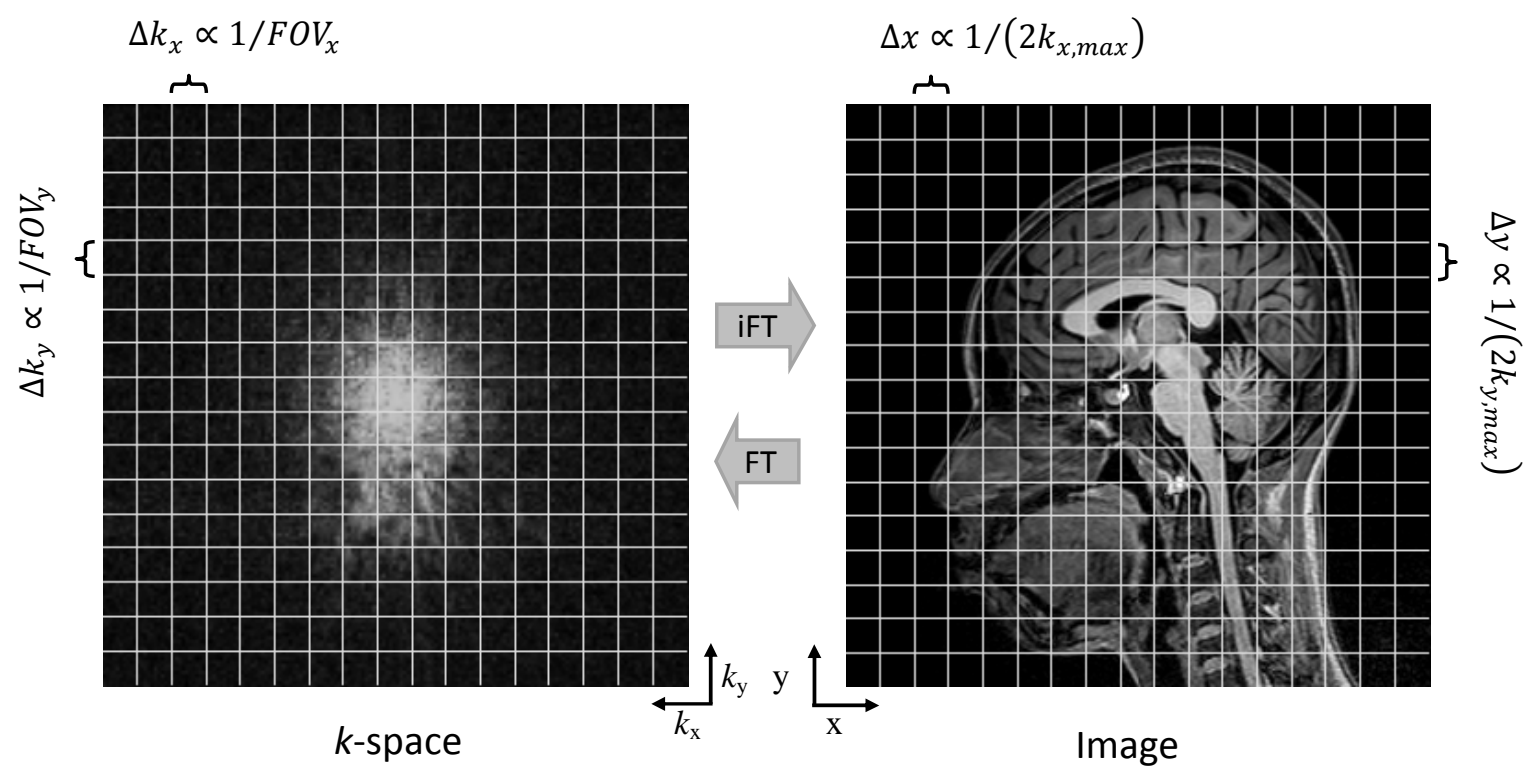

Figure 2.1 Relationship between the $k$-space and the image domain.

\subsubsection{Sampling Requirements}

During the presence of the magnetic gradient field, the sampled signal is encoded along the respective $k$-space positions. By iFT of the $k$-space data points, an image is reconstructed. Therefore, MRI is essentially Fourier imaging and most MR image characteristics can be deduced from the mathematical properties of the Fourier transform. This implies that certain requirements should be fulfilled during the acquisition according to these properties in order to reconstruct the image properly without artifacts. Such basic requirements are introduced here while a mathematical description of the Fourier transform and Nyquist criterion of signal sampling for MRI is given in Appendix C.

In an actual measurement only a finite number of $k$-space points can be acquired because the object is sampled discretely at a certain sampling rate. Thus, a $2 \mathrm{D} k$-space is made up of a grid of points. In addition, because discrete sampling leads to occurrence of periodic object 
copies and the distance between the copies is reciprocal to the sample distance, by applying the Nyquist criterion, the distance between each point in the $k$-space, i.e. the sample distance $\Delta k$, has to fulfill the relation to the chosen field-of-view (FOV) in one measurement:

$$
\Delta k \leq 1 / F O V
$$

In general, if the sample distance is too large or if there is any spin density existing outside the FOV, then the neighboring copies overlap with each other in the image domain. This so-called aliasing will cause fold-over or wrap-around artifacts in the image.

Besides, the number of samples $N_{s}$ is referred to as base resolution or image matrix size because it is directly related to the number of image pixels. Therefore, the spatial resolution $(\Delta d)$ of the reconstructed image is given by the following relation:

$$
\Delta d=F O V / N_{s}
$$

The relationship between image dimension and $k$-space parameter is shown in Fig 2.1.

\subsection{Sequence Type and Contrast Mechanism}

In contrast to NMR spectroscopy, MRI does not use the FID signal directly for imaging, but instead uses an echo, which is generated by the rephasing process of the transverse magnetization during the recovery of the signal intensity. This is done by controlling the timing of RF pulses and gradient fields through imaging pulse sequences that contain hardware instructions. The typical timing parameters are $T R$ (repetition time) and $T E$ (echo time). $T R$ refers to the time between the RF excitation pulses and $T E$ is the time between the RF excitation pulse and the detected signal (echo) produced by the sample.

Intrinsically, MR signal intensity and image contrast, i.e. the image pixel value, is governed mainly by three parameters: the tissue spin density, T1 and T2 relaxation times [34]. However, by experimentally varying the pulse sequence timing, i.e. extrinsic factors such as $T R$ and $T E$, differences in the magnetic relaxation properties of tissues can be emphasized, and spin density, T1 and T2 information can be individually accentuated in the resultant image as the predominant source of contrast [35]. This advantage of MRI inevitably makes it superior to, for example, X-ray or CT in which the source of contrast arises solely from the physical density. In the rest of this section, the basic pulse sequences are introduced. 


\section{Spin Echo Technique}

The spin echo (SE) technique was introduced as early as 1949 with the discovery of the spin echo by Erwin Hahn [36]. Usually, it has two RF pulses - a $90^{\circ}$ excitation pulse and a $180^{\circ}$ refocusing pulse. For MRI, the former is applied to a selected slice of tissue that rotates the magnetization vector into the transverse plane. The induced FID signal decays with a time constant $\mathrm{T}_{2} *$, which reflects both spin-spin relaxation (T2) and relaxation caused by nonuniformities in the field. After some time (TE/2), a $180^{\circ}$ pulse is applied which reverses the signal decay (dephasing) caused by field inhomogeneities and restores the magnetization vector in the transverse plane. An echo signal, i.e. spin echo, is produced when the spins are refocused (at time $T E$ ) and is measured in the presence of a readout gradient $\left(G_{R}\right)$ during which data samples $\left(N_{s}\right)$ are digitized for storage in the acquisition computer by the analog-todigital converter (ADC).

A very long $T R$ is necessary since time must be allowed for nuclei of tissues to undergo complete longitudinal relaxation to yield high signals on subsequent excitations. The signal intensity is then independent of $\mathrm{T} 1$ because the spins used for imaging are fully relaxed before the next excitation pulse is applied. Therefore, SE technique gives T2 weighted contrast. If the $T R$ is shortened, the tissue will have incomplete longitudinal relaxation, the signal intensity will vary with $\mathrm{T} 1$, and the image will contain some $\mathrm{T} 1$ weighting.

The sequence diagram of a basic SE technique is shown in Fig 2.2. Several advanced imaging pulse sequences such as rapid acquisition with relaxation enhancement (RARE) belong to this technique.

\section{Stimulated Echo Technique}

The stimulated echo (STE) pulse sequences $[37,38]$ are based on the properties of stimulated echo first identified by Hahn [36] and further quantified by Woessner [39]. They consist of at least three RF pulses with typical $F A$ s of $90^{\circ}$, between which strong dephasing gradients are applied. Considering the typical case where three unequally spaced $90^{\circ} \mathrm{RF}$ pulses are applied, besides three conventional SEs resulting from each possible pair of RF pulses and one secondary SE resulting as a reflection of the first SE by the third RF pulse, one stimulated echo is produced by the combined effect of all three RF pulses (Fig 2.2). After the first two $90^{\circ}$ RF pulses, only half of the spins are subsequently refocused into the spin echo in the transverse plane, while the other half contributes to T1 recovery in the longitudinal direction. By the third RF pulse this "stored" magnetization is then flipped into the transverse plane 
which forms the STE. It appears at a time delay after the third RF pulse equal to the interval between the first two RF pulses. Although classically produced with $90^{\circ}$ pulses, any RF pulses other than an ideal $180^{\circ}$ can produce a stimulated echo.

Since all echoes are RF refocused, like SE sequences, the STE technique is robust to susceptibility artifacts. Besides, lower RF power is deposited when compared to SE sequences, because of the absence of the $180^{\circ} \mathrm{RF}$ pulse. However, because the maximum STE amplitude is only $50 \%$ of a conventional SE, STE images have noticeably lower SNR.

The STE technique, under the acronym STEAM (stimulated echo acquisition mode), is also widely used in diffusion imaging [40-43] arising from the early measurement [44], and routinely employed in MR spectroscopy as a localization method to select a voxel from three orthogonal imaging slices [45-47].

Spin Echo

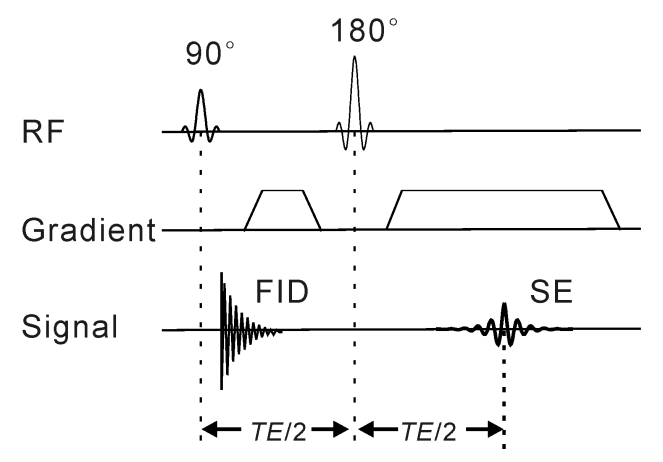

Stimulated Echo

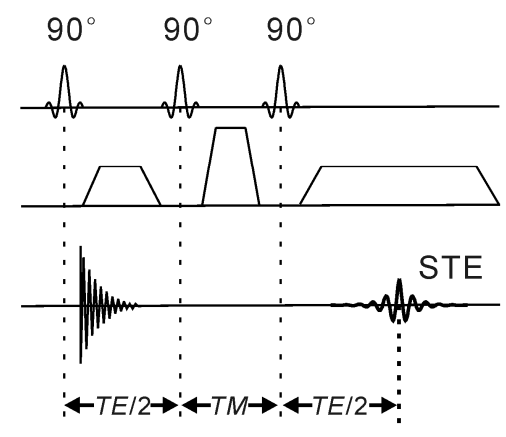

Gradient Echo

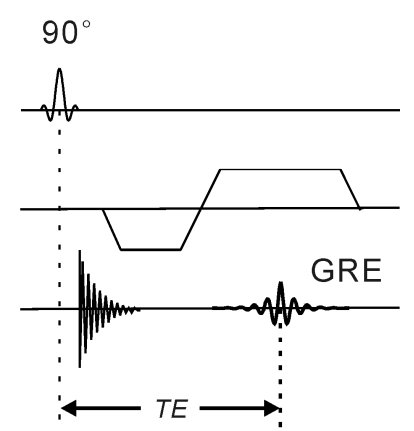

Figure 2.2 Pulse sequence timing diagram of the basic spin echo (SE) technique (left), simulated echo (STE) technique (middle), and gradient echo (GRE) technique (right). $T E$ is the time between the middle of the excitation pulse and the maximum of the detected echo; $T R$ is the time between successive excitations; $T M$ is the time between the second and the third RF pulse; FID is the free induction decay. The inversion of the rotating direction by a $90^{\circ}$ or $180^{\circ}$ refocusing pulse corresponds to a reflection of the $k$-space position, i.e. a change in the coordinate sign, so the readout gradient should be switched in the same direction as the prephasing gradient, which is different from the switching of the dephasing gradient in gradient echo techniques.

\section{Gradient Echo Technique}

The gradient echo (GRE) technique, being described in the early 60s [48, 49], was the archetypal Fourier imaging technique as spin warp imaging [50]. It consists of one single RF pulse (often called the $\alpha$ pulse) for excitation and does not use $180^{\circ}$ pulse to refocus the spins. Instead, a gradient is applied after the initial $\alpha$ pulse that induces spin dephasing, immediately after which a second gradient pulse of the same duration and magnitude but opposite polarity is applied to reverse this dephasing and produce the echo signal, i.e. gradient echo. As in the spin echo technique, the GRE sequence is repeated after one $T R$ for successive acquisition to 
cover the $k$-space for image reconstruction.

The absence of the $180^{\circ} \mathrm{RF}$ pulse in GRE sequences has several important consequences. First, a shorter $T R$ may be applied than an analogous spin echo sequence, which leads to shortened overall imaging time. Second, less RF power is applied so that the total RF energy deposition to the patient is lower. Additionally, T2* weighting is allowed because the static sources for spin dephasing - unavoidable $B_{0}$ inhomogeneity and magnetic susceptibility differences - also contribute to the signal decay as they are not rephased. But for this reason, the overall signal level in GRE images is less than for SE images with comparable acquisition parameters, and the GRE image is also more sensitive to susceptibility differences caused by metal implants or air-tissue interfaces.

The basic sequence diagram of a GRE technique is shown in Fig 2.2. Numerous advanced imaging pulse sequences such as fast low angle shot (FLASH) belong to this technique.

\section{Proton Density Contrast}

Because the magnitude of the received signal is proportional to the magnetization, which is the summation of the proton density (spin density) in one voxel, tissue with a high proton density (PD) reaches a high level of magnetization and thus induces a strong signal while tissues with lower PD induce a low signal. Therefore, MR image is subject to a basic and direct weighting with the proton density. For instance, in proton density weighted (PDW) images of the brain, as illustrated in Fig 2.3, gray matter and cerebrospinal fluid (CSF) are brighter than white matter. To obtain a pure PDW images, the signal has to be acquired in a way that any additional signal modulation from relaxation or saturation is avoided. This is achieved by minimizing the duration between the RF excitation and the data readout, i.e. $T E$, and keeping a rather long repetition delay, i.e. $T R$, to allow for a homogeneous recovery of the longitudinal magnetization. In practice, however, it is often infeasible either to wait for a complete recovery of the longitudinal magnetization because of the unbearable measurement time, or to have a short enough $T E$ because the RF excitation pulse and data readout themselves take some time. Therefore, the proton density can only be approximately determined in this case. Nevertheless, it is sufficient for many diagnoses. 


\section{T2 Contrast}

A contrast weighting with the $\mathrm{T} 2$ relaxation time can be achieved by increasing $T E$, so that an intended delay of the signal is introduced between the excitation and the signal detection. In such a case, the signal strength depends on the T2 relaxation. For instance, tissues with a small T2 value have faster signal decline and therefore appear dark in the image, e.g. white matter, while more signal remains in tissues with a large T2 value which appear bright, e.g. CSF and grey matter (Fig 2.3). The SE technique creates T2 weighted (T2W) images which depend only on the random dephasing process due to spin-spin interactions, because the systematic dephasing due to field inhomogeneity is rephased. Therefore, images are not sensitive to off-resonance effects caused by local inhomogeneity of the magnetic field or susceptibility differences arising at air-tissue interfaces. For clinical diagnosis, T2 contrast is often used because it is very sensitive to malignant changes of the tissue and is helpful for, e.g. cancer detection.
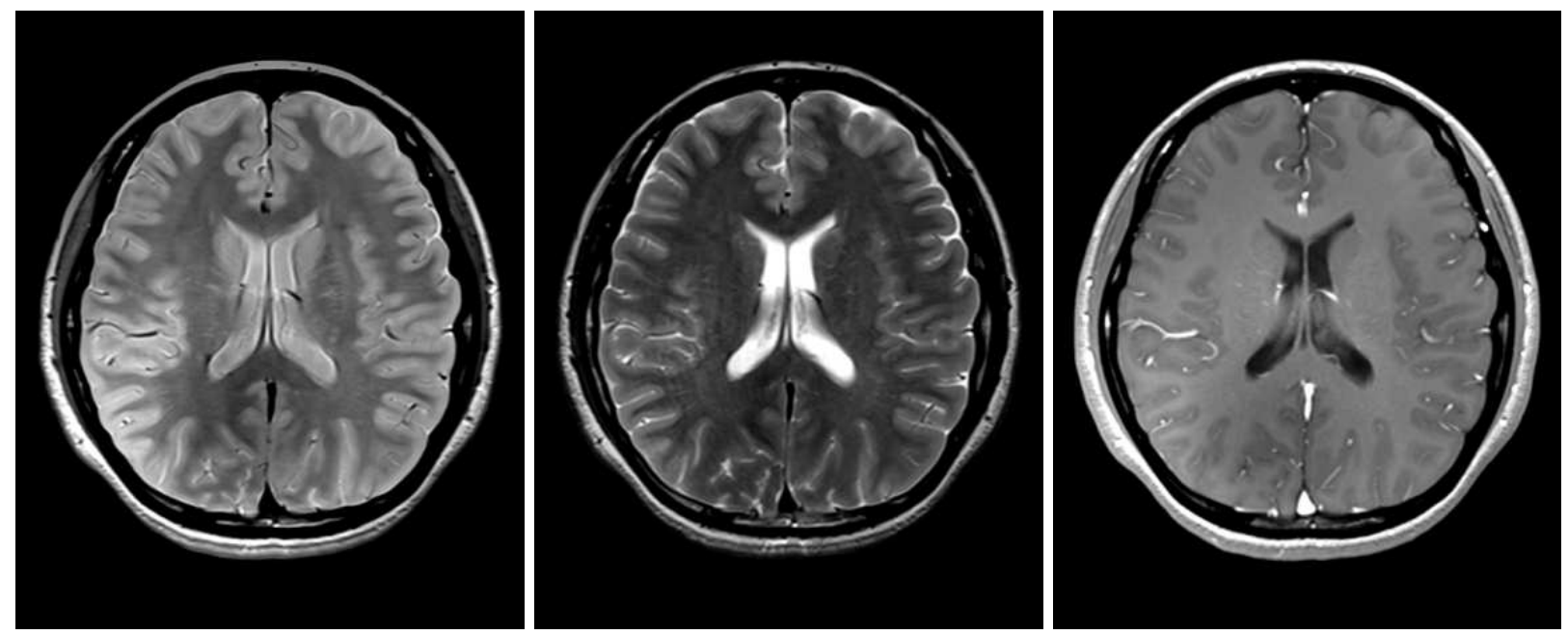

Figure 2.3 Basic MRI contrast due to proton density (left), T2 (center), and T1 (right). The three images were acquired from a human brain in a transversal plane. Contrast is shown for the white and grey matter, the lateral ventricles with cerebrospinal fluids, and the sinus. For details of the anatomical structures and the interpretation of MRI brain images refer to [51].

If, however, both mechanisms of the loss of the phase coherence are taken into account, as it may be achieved in GRE techniques, signal amplitude is then dependent on the effective T2 relaxation time and image contrast is thus $\mathrm{T} 2 *$ weighted. In practice, for example, the functional MRI (fMRI) techniques utilize the spin dephasing caused by the local change of the hemoglobin concentration to study functional brain activations in response to the stimulus. 


\section{T1 Contrast}

A contrast weighting with the T1 relaxation time can be obtained by performing fast repetitive excitations with incomplete recovery of the longitudinal magnetization at short $T E$. In such a way, the signal amplitude becomes dependent on the T1 relaxation. If tissues have long T1, then only a small amount of the longitudinal magnetization is recovered at the end of the sequence and available for a next excitation. As a consequence, such tissues create a weak signal in the following measurements of the sequence, which is referred to as spin saturation effect. On the contrary, tissues with small T1 induce a strong signal, because a high amount of longitudinal magnetization is used in the subsequent excitations. Therefore, tissues with short T1 values that produce higher magnetization appear bright in the T1-weighted (T1W) images, which have an inverse character in comparison to the T2W images. For instance, the CSF, which has a long $\mathrm{T} 1$ and a long $\mathrm{T} 2$ relaxation time, appears dark in the $\mathrm{T} 1 \mathrm{~W}$ contrast but bright in the T2W contrast (Fig 2.3). For morphological imaging of anatomical structures, T1W images are often used to provide a good contrast.

The GRE technique creates typical T1W images. In practice, however, because full longitudinal magnetization is always available during the first measurement by running the sequence, the initial signal is still independent from the T1 value and only PD weighted. Therefore, it is necessary to drive the magnetization into a steady-state before data recording. This can be done with several preparation excitations.

\subsection{MRI System}

A conventional MRI system consists of a magnet and a gradient system, a shimming system, an RF transmitter/receiver system with an excitation and receiver coil, a patient table, a reconstruction computer, and an operator console with display monitors. All experiments for this thesis have been conducted with a commercial whole-body MRI system (MAGNETOM Trio, A Tim System; Siemens AG, Erlangen, Germany), and all in vitro studied having been carried out with different phantoms, as shown in Fig 2.4.

The magnet, as the core component of the system, consists of solenoidal coils. The coils are contained inside a tank (cryostat) of liquid helium (cryogen). At the same temperature as liquid helium $\left(-269^{\circ} \mathrm{C}\right)$, coils made of a niobium-titanium alloy become superconductive, i.e. flow without resistance, and thus without heat production. The strength of the main field $B_{0}$ is $2.89 \mathrm{~T}$ (the Earth's magnetic field is approximately 3 to $6 \cdot 10^{-5} \mathrm{~T}$ ) and the bore (with casing) 
has a length of $142 \mathrm{~cm}$ with a diameter of $60 \mathrm{~cm}$, allowing for FOV up to $50 \mathrm{~cm}$ in each direction. To achieve homogeneity of the static magnetic field, the shimming system (active or passive shimming or both) is employed. A detailed description is beyond the scope of this work, but the crucial importance of such homogeneity, i.e. the level of the uniformity of the field, should be known because the more uniform the static field is, the less the resonant frequency varies with position. Normally such field inhomogeneities can cause many difficulties in imaging, from image distortion to blurring and replication artifacts.
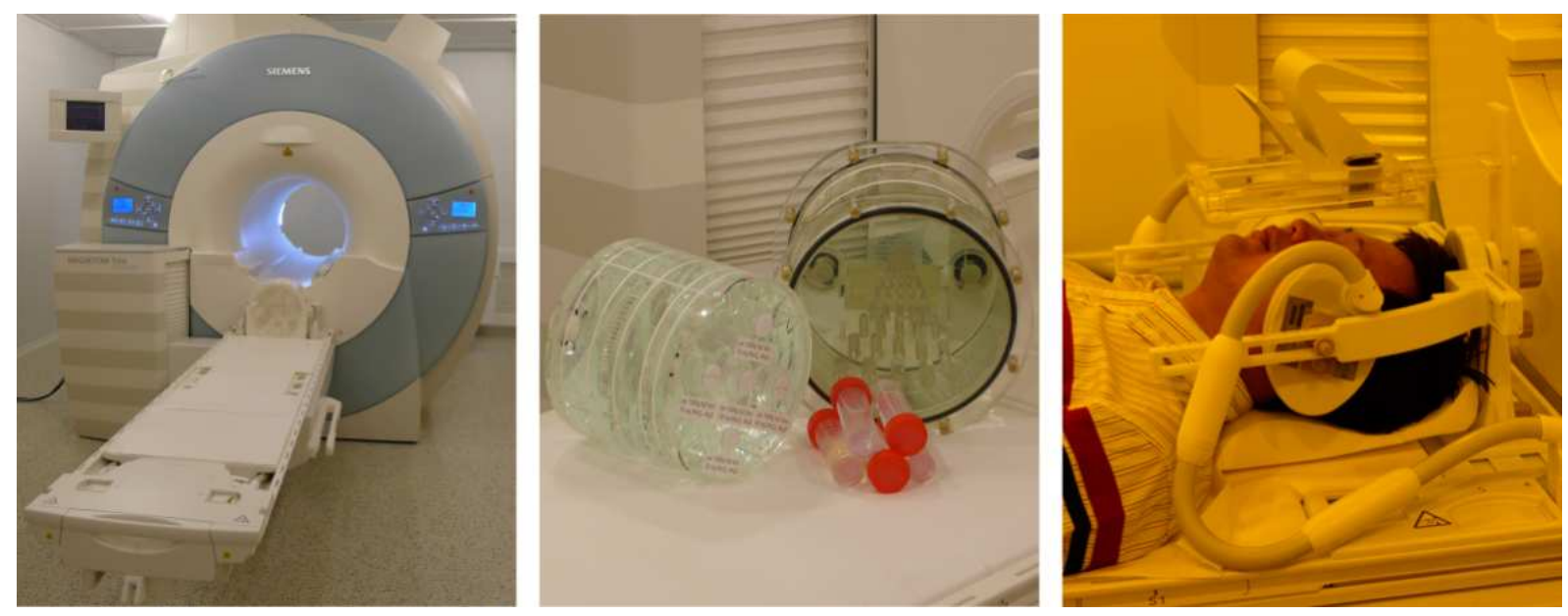

Figure 2.4 MRI system used in this thesis (left), different types of phantom for in vitro studies (middle), and an in vivo study of volunteer (right).

The gradient system for spatial encoding provides a maximum gradient field strength of 38 $\mathrm{mT} / \mathrm{m}$ per axis, switchable on a $10 \mu$ s raster with a maximum slew rate of $170 \mathrm{mT} /(\mathrm{m} \cdot \mathrm{ms})$.

The RF system uses separate coils to transmit RF pulses for excitation and to receive RF signals for data acquisition. The excitation is done with a body coil, which is mounted to the casing of the bore, and different coil types depending on the application are used to receive the resonance signal. 


\subsection{Summary}

The principle of MRI is based on nuclear spins. While the quantum nature of a spin in a strong static magnetic field determines the fundamental properties, an ensemble of spins can be modeled using classical mechanics. This latter approach serves to describe the application of RF pulses and magnetic field gradients as well as nuclear relaxation effects. The signal observed from the spin system during the relaxation may be detected with the use of a receiver coil and, for MRI, sampled through a $k$-space description. Depending on the spatial encoding of the $k$-space and its sampling during data acquisition, an image may be reconstructed through various reconstruction algorithms, which in most cases involve an inverse Fourier transformation.

As a medical tomography with numerous physical, mathematical and engineering concepts, MRI is much more sophisticated than CT or ultrasound. Any flaw during the imaging procedure, for example, undesired subject interference (e.g. motion), improper data sampling, imperfect instrument control during encoding, or mishandling of the acquired data during decoding, may result in images that are unable to properly represent the original subject (due to presence of induced artifacts or distortion), and may cause misinterpretation in clinical diagnosis. This is even more critical for RT-MRI, which requires the MRI procedure to be accomplished within a certain time without any unnecessary or even detectable delay, but still has to achieve high fidelity to the original subject or the dynamic phenomenon. 


\section{Chapter 3}

\section{Development of Real-time MRI: Physical Basis}

MRI is intrinsically a slow imaging method because of the following reasons: 1) signal acquisition has to be done by exciting the spins many times to maintain signal strength, when the transverse magnetization decay according to $e^{-t / T_{2}^{*}}$ is accounted for ( $\mathrm{T}_{2}^{*} \approx 20 \mathrm{~ms}$ ); 2 ) a certain time depending upon the desired contrast has to be spent to restore the thermal equilibrium before each excitation; 3) such process has to be repeated successively to cover the $k$-space with sufficient density and extent to properly reconstruct images of an object of a certain size with a given resolution. The total imaging procedure of conventional Fourier MRI - through such sequential and substantive repetitions of excitation, encoding, and waiting - is inherently time consuming, and therefore, specific ideas and proper physical strategies are needed for the development of RT-MRI [52].

Based on the basic echo generation methods described in the last chapter, many advanced techniques have been invented for acceleration of the imaging process. These fast imaging pulse sequences attempt to manipulate magnetic field gradients and RF pulses as rapidly as possible, and to cover the $k$-space in a very short period of time. For example, more than one $k$-space phase encoding line can be acquired by recording multiple echoes that are produced after one RF excitation. The signal acquisition time of such methods is many times shorter when compared to single-echo methods.

In this chapter, different principles of reducing scan time by fast pulse sequences are explained and comparatively evaluated, including the fast gradient echo approach, i.e. the FLASH technique. Respective variants are proposed and further improved as the first step to develop RT-MRI. 


\subsection{Signal Acquisition with the FLASH Technique}

A typical fast gradient echo technique, under the acronym FLASH (fast low-angle shot), uses slice-selective low flip angle RF pulses ( $\alpha$ pulses) with short repetition time $[16,53]$. In this manner, magnetization recovers rapidly due to low flip angle and no additional time needs to be spent before the next excitation. At the same time, an equilibrium, i.e. steady state, is established after a few initial $\alpha$ pulses between the magnetization reduction (due to excitation) and recovery (due to relaxation) because of the short $T R$ time constant.

Compared to the spin echo technique, the FLASH sequences are more sensitive to field inhomogeneities and susceptibility differences. However, they show several advantages: First, the shorter $T R(\leq 20 \mathrm{~ms})$ reduces the image acquisition time $(T A)$ to the order of seconds:

$$
T A=N_{A V E} \cdot T R \cdot N_{P E}
$$

where $N_{P E}$ is the number of total phase encodings steps and $N_{A V E}$ is the number of signal averaging, which for RT-MRI should be one. Second, considerably less overall RF power, i.e. much lower specific absorption rate (SAR), is deposited in the patient by eliminating $180^{\circ}$ refocusing pulses. Third, extra contrast due to in-phase and opposed-phase [54] conditions for fat and water signal is enabled without additional magnetization preparation pulses, which will be presented in Section 5.1, while in FSE or SE imaging, echo refocusing by $180^{\circ}$ pulse does not allow this phenomenon to be demonstrated. In addition, the FLASH technique shares the typical inflow effect from the basic GRE sequence - produces images characterized by bright signal intensity from moving fluids, e.g. rapidly flowing blood, compared to dark intensity of stationary fluids.

Although numerous fast GRE signal acquisition sequences have been developed based on the initial FLASH technique, the resulting images give rise to completely different contrasts. They are classified mainly according to the way the gradients are switched between consecutive excitation pulses and the resulting situation of the steady state, so-called steady state free precession (SSFP) [55]. The three classifications are outlined below while a detailed description may be found in $[56,57]$.

\subsubsection{Spoiled Steady-State Free Precession (SSFP)}

Because of the short $T R \mathrm{~s}$ that are used, the dephasing of the transverse magnetization is incomplete. In the conventional spoiled FLASH technique, the steady state is maintained but 
the residual net transverse magnetization is purposely destroyed by applying either spoiler gradients with different strength for each phase-encoding step before the next $\alpha$ pulse (known as gradient spoiling) [45] or by changing the phase of the RF pulse for each transmit-receive cycle (known as RF spoiling) [58]. In general, due to completely destroyed residual transverse magnetization, only the regular FID signal is acquired by the spoiled FLASH sequence, which leads to T1W images.

\subsubsection{Refocused SSFP}

A typical refocused FLASH technique, known as gradient-recalled echo in the steady state (GRASS) sequence, is very similar to the spoiled FLASH sequence except for the "rewinding" of the phase-encoding gradients $[45,59]$. In such a way, the overall phase of the transverse magnetization is maintained constant from one repetition to the next [60-62] and a steady state of both transverse and longitudinal magnetization is established. Since the residual transverse magnetization rephased by subsequent RF pulses produces stimulated and spin echoes, which are included with gradient echoes, a SSFP-FID signal, differing from regular FID, is acquired by refocused FLASH.

For small flip angles, image contrast of refocused FLASH is very similar to that of spoiled FLASH. At relatively larger flip angles and short $T R$ s (i.e. $T R \ll \mathrm{T} 2$ ), the signal contrast is related to T2, causing structures with long T2, such as CSF, to become bright. For larger flip angles and long TRs, the contrast becomes T1-weighted, similar to spoiled FLASH images again, because the long $T R$ naturally spoils the transverse magnetization.

\subsubsection{Balanced SSFP}

A balanced SSFP technique, originally termed fast imaging with steady-state precession (FISP) [63], also called TrueFISP, is a modification of the refocused SSFP sequence in which all three gradient axes (frequency, phase, and slice) are refocused or balanced [64-67]. Since both of the stimulated and spin echoes made up of the residual transverse magnetization are produced by equidistant RF pulses, in addition to the just created FIDs, the overlapping gradient echoes of the SSFP-FID and SSFP-echo signal is acquired. Under such fully balanced conditions, blood and even CSF with slow flow have high signal intensities, similar to heavy $\mathrm{T} 2$ weighting. 
Like in the refocused SSFP sequence, the steady state in the balanced case is maintained by using relatively higher flip angles to produce excellent SNR. It gives good blood and muscle (myocardium) contrast, and is therefore widely used in cardiac imaging [68-70]. However, its high sensitivity to off-resonance effects limits the use in many situations, particularly at high magnetic field strengths of 3 Tesla. Preliminary results of the balanced SSFP are included in Fig 3.1 of the next section, whereas more detailed experimental studies of all three variants are described and discussed in Chapter 6.

\subsection{Comparison of Available Acquisition Techniques}

In addition to the FLASH techniques, the most obvious way of rapid signal acquisition is to generate multiple encodable signals (e.g. involving SEs, STEs or GREs) per repetition interval. This principle is used in many other fast pulse sequences. As they diverse in detail, these important techniques are carefully compared in theory and experimentally, in order to find an optimal solution for RT-MRI.

\subsubsection{Brief Review}

\section{Fast Spin Echo Sequence}

The fast spin echo (FSE) sequence, originally termed rapid acquisition with relaxation enhancement (RARE) technique [17], relies on the Carr-Purcell-Meiboom-Gill (CPMG) condition $[71,72]$. It uses a long train of $180^{\circ}$ refocusing pulses after an initial $90^{\circ}$ excitation pulse to generate a string of multiple spin echoes. It is also called turbo spin echo (TSE) sequence, and the number of echoes received in the same repetition is called turbo factor or echo train length $(E T L)$. Each echo in the train is phase encoded to generate a different line in the $k$-space so that a rapid acquisition time is achieved by filling multiple lines in one repetition $(T R)$ :

$$
T A=T R \cdot \frac{N_{P E}}{E T L}
$$

The contrast of FSE images is primarily T2 weighting. As echoes are received at different echo times, the echoes corresponding to the central $k$-space lines are the ones that determine image contrast and the time point at which they are acquired is called effective TE (TE eff), 
which can be selected for stronger T2-weighting (T2W) or proton density-weighting (PDW). Certain magnetization preparation pulses can be combined with FSE sequences [50, 73]. An inversion time $(T I)$ between the previous $180^{\circ}$ inversion pulse and the following $90^{\circ}$ pulse can selectively suppress fat signals (as the STIR technique [74] and the FLAIR technique [75]) due to their different $\mathrm{T} 1$ relaxation times.

The advantage of FSE techniques is that it efficiently reduces the acquisition time of the standard SE method to acceptable limits for clinical practice (on the order of minutes), particularly in obtaining strongly T2W or PDW images. It is also immune to off-resonance distortions from field inhomogeneity and tissue-induced susceptibility variations, which are cancelled by the $180^{\circ}$ refocusing pulses. However, the use of early echoes with strong intensity to fill the high spatial frequencies in the $k$-space periphery is likely to produce truncation or Gibbs artifacts, while the use of late echoes with weak intensity may result in a blurred image with poor spatial resolution. Additionally, since the long $T R$ is still kept, the emphasis of FSE technique is on overall efficiency and diagnostic accuracy in clinical applications, e.g. by increasing the in-plane resolution while maintaining conventional T2 contrast, rather than on rapid signal acquisition.

An extreme case of FSE, so-called half-Fourier acquisition single-shot turbo spin-echo (HASTE), which uses only one initial preparation pulse followed by a very long echo train to record signals (i.e. $T A=T R$ ), is a true ultra-fast pulse sequence. It is combined with partial Fourier phase encoding (PF), which utilizes the complex conjugate symmetry property of $k$ space and omits the acquisition of certain phase-encoding steps or even half of the $k$-space except for a few central lines for phase correction [18, 76, 77]. Such a single-shot approach enables the acquisition of good motionless images within a single breath hold, e.g. in abdominal or lung imaging. However, the signal acquisition with very long echo train results in SNR decay and restricted spatial resolution, and also blurring in the phase encoding direction, as shown in Fig 3.1. Furthermore, the large quantity of RF energy deposited by $180^{\circ}$ pulses, which manifests as heating, fundamentally limits the application of such sequence type, although a certain reduction could be achieved by lower flip angles for refocusing [78, 79], particularly in real-time imaging when longer spin echo train length is desired for shorter acquisition time.

\section{Fast Stimulated Echo Sequence}

The fast stimulated echo technique, so-called turbo-STEAM, replace the third $90^{\circ} \mathrm{RF}$ pulse in 
the basic stimulated echo sequence (STEAM) [37] by a series of readout RF pulses with flip angles (FAs) lower than $90^{\circ}$ [80]. In such a way, after the magnetization is prepared by the first two RF pulses, the rapidly repeated readout RF pulses continuously tip a certain amount of magnetization into the transverse plane, and the corresponding stimulated echo train is then differently phase encoded similar to the FSE technique but only on the order of a second. For example, the TA for a single-shot turbo-STEAM is given by:

$$
T A=T E / 2+T M+T R \cdot N_{P E}
$$

where $T M$ is middle time between the second and the third RF pulses. However, unlike SE signals in the FSE technique that are attenuated by T2 relaxation, the individual STE signals experience T1 weighting.

The advantage of this fast STE technique is that, because all acquired echoes are RF refocused, the resulting images are insensitive to resonance offset effects from chemical shifts or magnetic susceptibility differences. Moreover, it has a much lower RF power deposition in comparison to FSE sequences.

However, the obtained image with low level of signals remains a persistent problem although a few approaches may be applied to enhance the available SNR, for example, by combining partial Fourier phase encoding with single-shot STEAM [81-83] to reduce the number of necessary excitations [84], or by employing variable flip angles and using multishot STEAM with segmented $k$-space acquisition [85], as the results shown in Fig 3.1. In particular, for real-time imaging when the $T A$ of one slice is already shortened to achieve high speed by compromising certain SNR, additional signals degradation would be a fatal problem.

\section{Echo Planar Imaging Sequence}

The general concepts of echo planar imaging (EPI), both gradient and spin echo variants, were described originally by Mansfield [15, 86], and improved later as a standard sequence mainly for fMRI [87-89]. Typically, a single-shot EPI acquires all image data during a single $T R$ period. Immediately following a slice selective $90^{\circ} \mathrm{RF}$ pulse, the phase-encoding gradient is blipped on and off during continuous reversal of the frequency-encoding gradient. Each gradient echo is separately phase encoded and the $T E_{\text {eff }}$ is determined by the time to the central gradient echo. It is considered as an extreme case of single-shot fast GRE sequence.

A distinct advantage of EPI is that an entire image can be obtained in just $100 \mathrm{~ms}$ because only one (i.e. $T A=T R$ ) or two RF excitation pulses are required. However, sampling all of $k$ space after a single excitation requires not only extremely fast sampling with wide receiver 
bandwidth, but also high amplitude and rapid slew rate of gradients. Although the current available gradient systems in commercial MRI scanners are already strong and fast enough, the rapid time-varying magnetic fields demanded by EPI as well as by balanced SSFP GRE (TrueFISP) may produce peripheral nerve stimulation (PNS) and muscle spasms in extreme cases through Faraday's law of induction, and thus possess potential hazards to the patient. In addition, the limitation on the number of signals to be acquired before the magnetization dephases due to $\mathrm{T} 2$ relaxation also limits the spatial resolution.

Moreover, due to relatively long $T E$, dephasing induced by susceptibility differences may cause geometric image distortions and local signal void at interfaces of bone, air, and brain [90]. Although it is possible to reduce the strength of the distortions to some degree by applying a $180^{\circ}$ refocusing pulse, so that the sampling of the $k$-space center corresponds to a spin echo (SE-EPI), it remains a problem [91]. EPI is also very sensitive to ghosting artifacts. Because the signal is sampled with alternating gradients that are every other line reversed, any imperfection during the entire signal acquisition can modulate the signal at half the Nyquist frequency, which causes an "N/2" ghost in the phase-encoding direction.

\section{Hybrid Echo Sequence}

The gradient- and spin-echo (GRASE) technique [92, 93], also called turbo gradient spin echo (TGSE) sequence, is a hybrid sequence, which combines $180^{\circ} \mathrm{RF}$ refocusing pulses interleaved with refocusing gradients for signal acquisition. After a $90^{\circ}$ excitation pulse, $180^{\circ}$ RF refocusing pulses are used to create an echo train similar to FSE techniques, and between each pulse three or more gradient echoes, indicated by EPI factor, are created by a combination of stepped phase-encoding gradients and switched frequency-encoding gradients. It is equivalent to multi-shot spin-echo EPI, with typically interleaved or segmented phase encoding lines in the $k$-space. The imaging time of one slice (TA) is given by:

$$
T A=T R \times \frac{N_{P E}}{E T L \times E P I f a}+T R
$$

where echo train length $(E T L)$ represents the number of repetitive $180^{\circ}$ pulses.

Because it contains both SEs and GREs, it is not difficult to deduce the characteristics of the GRASE technique: sensitivity to susceptibility differences is less than EPI but higher than FSE; RF power deposition is lower than FSE but higher than EPI; overall signal decay is determined by $\mathrm{T} 2$ rather than $\mathrm{T} 2 *$, which results in relatively higher achievable spatial resolution than EPI and comparable to FSE $[94,95]$. 
However, while the repetition of $180^{\circ}$ refocusing pulses reduces the phase errors due to field inhomogeniety and chemical shift in magnitude in general, they reproduce periodic phase modulations within an echo train. Because of the segmented $k$-space encoding, this may lead to ghosting artifacts. In addition, although GRASE efficiently combines advantages of EPI and FSE sequences, another disadvantage of it, shared by FSE sequences, is that magnetization remains saturated after a single shot, which requires a delay before the next acquisition [57, 96, 97]. Thus, the total image time of GRASE is not much reduced compared to the other fast acquisition techniques.

\section{Turbo-FLASH Sequence}

This FLASH-based ultra-fast GRE sequence uses an inversion pulse to "prepare" the magnetization to selectively emphasize desired contrast during the rapid imaging [98]. Normally an initial $180^{\circ}$ inversion pulse is executed [59], similar to inversion recovery (IR) technique, followed by a low $F A$ and short $T R$ gradient echo train to complete image acquisition in a very short time, i.e. less than $1 \mathrm{~s}$.

The advantage is its ability to provide very strong contrast between tissues having different $\mathrm{T} 1$ relaxation times or to apply specific suppression to tissues like grey matter as shown in Fig 3.1. Due to the very short acquisition time, both single-shot and multi-shot versions with segmented $k$-space encoding [99] over several cardiac cycles have been applied to breath-hold cardiac imaging [100, 101]. Besides, an extension of this technique results in magnetization-prepared rapid gradient echo (MP-RAGE) MRI for 3D imaging [59, 102-104].

However, the disadvantage is that the spatial resolution is limited for single-shot turboFLASH due to fast signal decay, and the additional inversion RF pulse makes this sequence less time efficient than the other pulse sequences, particularly for multi-shot versions when sequential images are to be acquired as in RT-MRI.

\section{BURST}

Burst excitation pulse imaging (BURST) technique [105, 106] utilizes a rapid equidistant sequence of nonselective RF excitations with very low $F A$ s (e.g. $\left.5^{\circ}\right)$ and a slice-selective $180^{\circ}$ refocusing pulse to generate a long train of multiple echoes accompanied by either constant or switched gradients [107]. An image is formed from a small number of repeats of this multiecho sequence segment with even sub-100 ms acquisition times possible. Related ultra-fast methods are DANTE [108] and DUFIS (or OUFIS) imaging [109, 110] which can similarly 
acquire images in about $10 \mathrm{~ms}$.

The advantage of this sequence type is that it is less demanding on gradient speed than other ultra-fast techniques (e.g. EPI) and it produces images substantially free of susceptibility artifacts. However, the major problem is its low SNR, which is caused by inefficient use of the available magnetization. Such a fatal disadvantage makes this technique less competitive to the other methods, and therefore has hardly been used as a fast scan technique. For the same reason, it is neglected here for further discussion.

\subsubsection{Experimental Comparison}

Experimental comparisons of these rapid signal acquisition techniques are presented in Fig 3.1. Results from in vitro phantom study were chosen from extensive systematic experiments and results from in vivo human brain study were chosen from the same volunteer (male, 28 years old) among five experiments with healthy volunteers ( 3 female, 2 male, age 29 \pm 1 ). All sequences were run with the fastest speed, i.e. possibly shortest $T R$ and $T E$, but with reasonable contrast to offer, so that the potential of each technique for RT-MRI can be explored.

Values of signal-to-noise ratio (SNR) and contrast-to-noise ratio (CNR) were measured from the acquired brain images with all regions of interest (ROIs) about $50 \mathrm{~mm}^{2}$. The SNR was defined as mean signal intensity in a uniform region (white matter) divided by standard deviation (SD) in a region of noise outside the object. For the CNR, the mean difference of the signal intensities between two areas (white matter and CSF) was calculated and divided by the SD of the noise measured outside the body tissue in a region free of artifacts at simple visual inspection.

In vitro measurements with phantom had an in-plane resolution of $1.0 \times 1.0 \mathrm{~mm}^{2}$, with a FOV of $256 \times 256 \mathrm{~mm}^{2}$ and an image matrix of $256 \times 256$, except for the images acquired by the signal-shot turbo-FLASH and EPI, which were limited to $1.0 \times 2.0 \mathrm{~mm}^{2}$ with half of the phase-encoding steps due to signal decay. To avoid such limitation and also the very low SNR, multi-shot segmented turbo-STEAM was used to achieve $1.0 \times 1.0 \mathrm{~mm}^{2}$ spatial resolution in the phantom measurement. However, the periodic phase errors across the $k$-space resulted in ghosting artifacts in the phase-encoding direction (black arrows). 

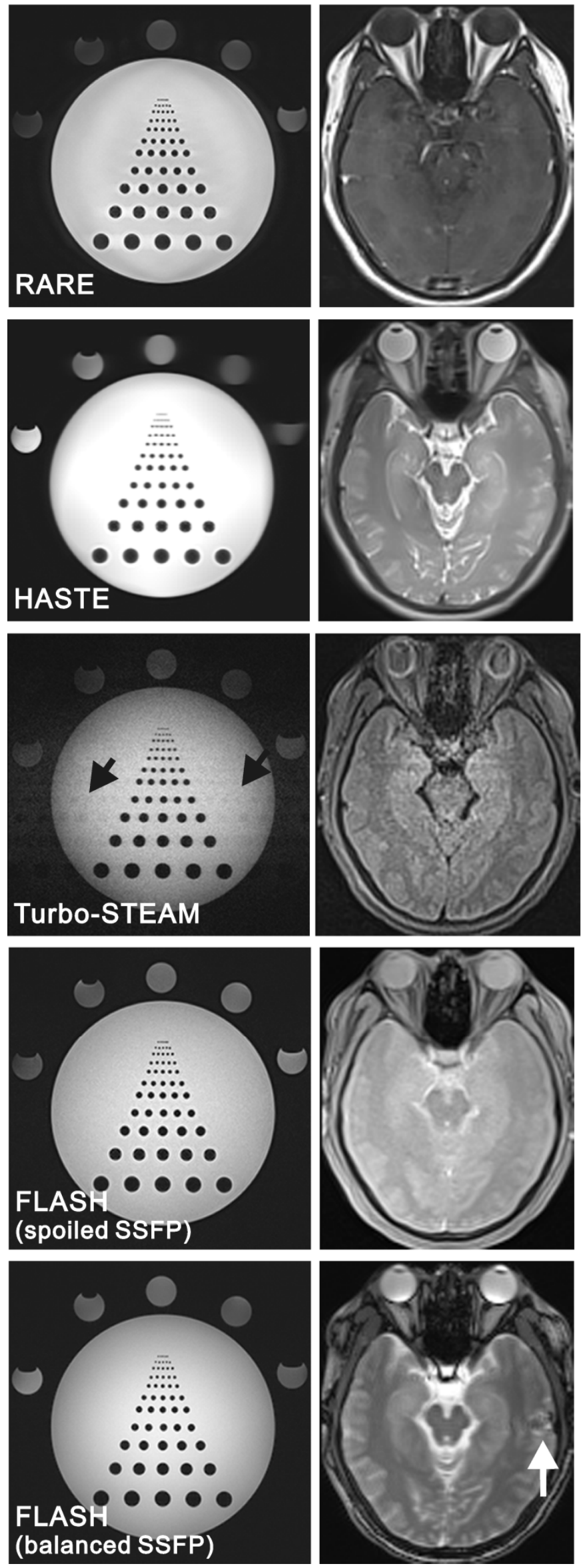

\section{TR/TE/TA \\ ( $\mathrm{ms} / \mathrm{ms} / \mathrm{s})$}

$240.0 / 6.2 / 4.0$

$97 / 8$

$500.0 / 24.0 / 0.5$

$183 / 23$

$10.0 / 10.0 / 4.0$

$78 / 14$

$12.0 / 1.8 / 1.0$

$394 / 105$

$3.6 / 1.8 / 0.3$

$578 / 218$ 

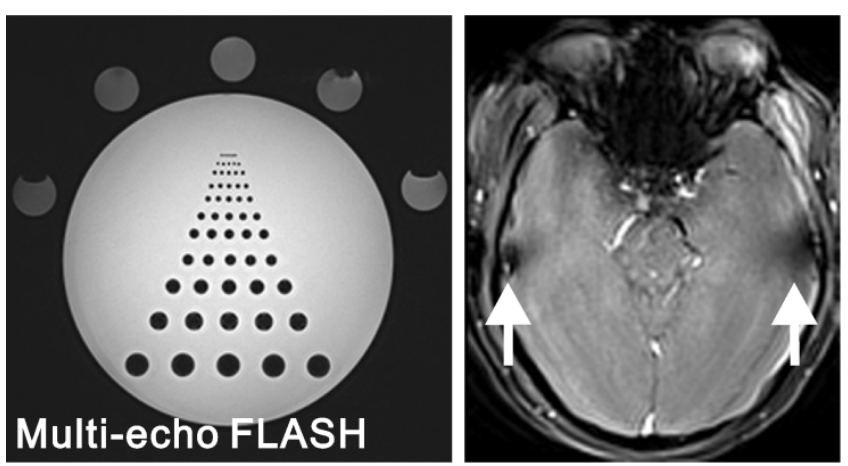

\author{
TR/TE/TA \\ (ms/ms/s)
}

SNR/CNR
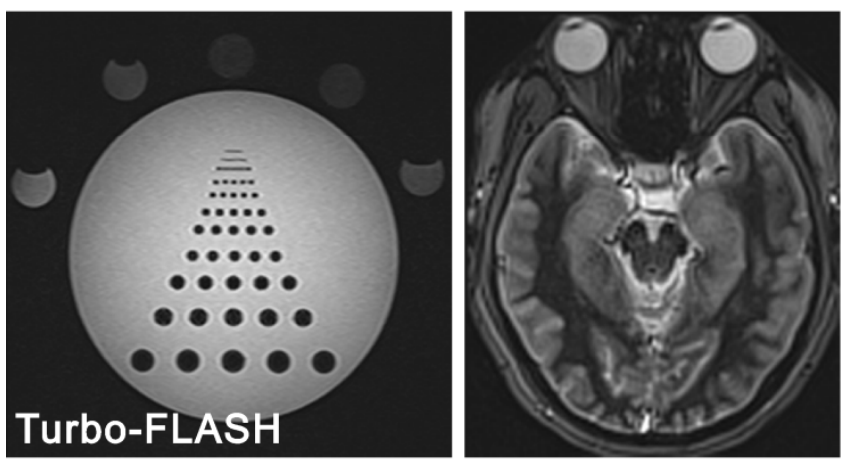

$500.0 / 24.0 / 0.3$

$271 / 55$

$800.0 / 2.8 / 0.8$

$119 / 85$
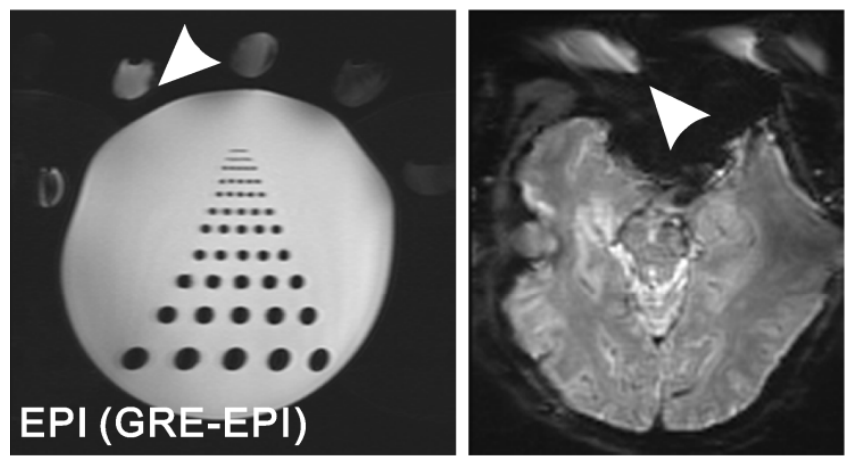

$200.0 / 37.0 / 0.2$

$138 / 42$
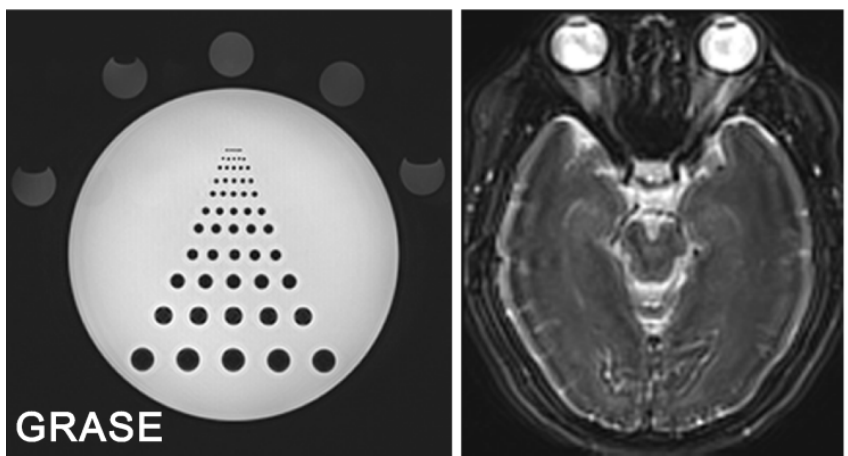

$550.0 / 55.0 / 5.0$

$137 / 49$

Figure 3.1 Experimental comparison of various rapid signal acquisition techniques. First column: phantom measurement; second column: human brain measurement. Black arrows in the turbo-STEAM phantom study indicate ghosting artifacts due to phase errors; white arrows in the balanced SSFP and multi-echo FLASH human brain studies indicate susceptibility artifacts due to off-resonance sensitivity; white arrow heads in the EPI studies indicate geometric distortion and signal loss due to off-resonance effects. Details are in the text. 
Echo

RARE

HASTE

's

Turbo-STEAM

STE

FLASH

(spoiled SSFP)

GRE

\section{FLASH}

(balanced SSFP)
$[a-G R E]_{\text {reg }}$

\section{Simplified Sequence}

$\left\{90^{\circ}-\left[180^{\circ}-S E\right]_{E T L}\right\}_{\text {rep. }}$

$90^{\circ}-\left[180^{\circ}-S E\right]_{E T L}$

MSh:

$\left\{90^{\circ}-90^{\circ}-[\alpha-S T E]_{r e p}\right\}_{\text {shot tsogm }}$

SSh:

$90^{\circ}-90^{\circ}-[\alpha-G R E]_{\text {rop }}$

$[\mathrm{a}-\mathrm{GRE}]_{\text {rep }}$

\section{General Characteristics}

(Speed/SAR/Continuity/Image Quality: Resolution, Artifacts, SNR)

- Not fast;

- Very high SAR;

- Delay for signal recovery;

- High resolution:

- High SNR

- Ultra-fast;

- Very high SAR;

- Blurring due to signal decay

- Fast;

- SSh: limited resolution with very low SNR;

- MSh: ghosting artifacts with low SNR

- Fast;

- No restriction between resolution and signal;

- Low SAR.

- Susceptibility artifacts only at long TE

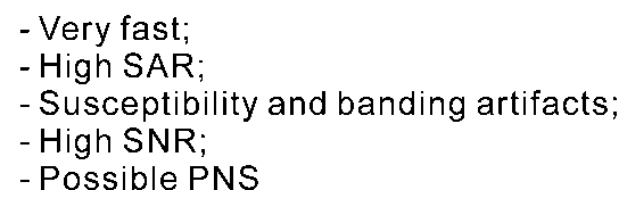

- Possible PNS 
$\begin{array}{lll}\text { Multi-echo FLASH } & \text { GRE } & \text { - Very fast; } \\ & \text { [a-GRE-GRE-... }]_{\text {segm. }} & \text { - Low SAR; } \\ & & \text { - Susceptibility \& ghosting artifacts }\end{array}$

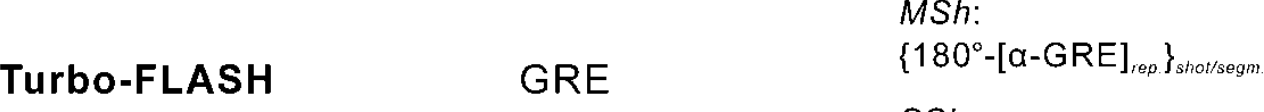

SSh:

$180^{\circ}-[\alpha-G R E]_{\text {rep }}$

MSh-GRE-EPI:

$\left\{90^{\circ}-\text { GRE-GRE-GRE-... }\right\}_{\text {sthotrep. }}$

$S S h$-GRE-EPI:

$90^{\circ}$-GRE-GRE-GRE-...

MSh:

GRASE

SE/GRE
- Very fast;

- SSh: Limited resolution \& contrast;

- MSh: ghosting artifacts \& time inefficiency due to interval time $(T I)$

- Very fast;

- SE-EPI: high SAR:

- SSh: limited resolution

- Susceptibility \& ghosting artifacts;

- Possible PNS

\section{- High SAR;}

- Delay for signal recovery

- MSh: not fast, with ghosting artifacts

SSh:

$90^{\circ}-\left[180^{\circ}-G R E-G R E / S E-G R E\right]_{E T / s e g m}$ 
In vivo measurements with human brain had a relatively lower in-plane resolution of $1.5 \times 1.5$ $\mathrm{mm}^{2}$, with a FOV of $192 \times 162 \mathrm{~mm}^{2}$ and an image matrix size of $128 \times 108$, to mimic the average spatial resolution desired in potential RT-MRI. The imaging slice was located in a transversal plane across the eyeballs. All images were acquired with a slice thickness (SLT) of $3 \mathrm{~mm}$ using a 32-channel head coil (Siemens AG, Erlangen, Germany).

The measured brain image as well as the calculated SNR and CNR from RARE sequence shows that the selected short $T R$ and $T E$ for rapid acquisition may lead to strong saturation of the spins and thus low SNR. Due to SAR limit, the FAs of RARE, HASTE and GRASE sequences had to be reduced from $180^{\circ}$ to $153^{\circ}, 135^{\circ}$ and $148^{\circ}$, respectively, during the experiments. Because of the very sensitivity to off-resonance effects, even small structures like the inner ear filled with air led to signal void (white arrow) in the balanced SSFP (TrueFISP) image, although it had highest SNR and CNR with a TA below one second. Similarly, multi-echo FLASH (like multi-shot GRE-EPI but with low FAs and segmented/interleaved $k$-space coverage) experienced the same susceptibility artifacts due to the accumulated phase incoherence during long TE. Single-shot EPI had the shortest acquisition time as expected, but continuously reversing the gradient polarity and a back-andforth path in the $k$-space coverage suffered from severe geometric distortion and also susceptibility artifacts (white arrow heads). Noticeably, the blurring in the HASTE phantom image due to fast signal decay and the "N/2" ghost artifacts in the gradient echo EPI phantom image are more obvious with original image size. Here for demonstration purpose, all images were scaled to provide a systematical overview. Only the generic FLASH technique (spoiled version as an example here) provided images free of artifacts or distortion in a short scan time with reduced TRs. Meanwhile, a good SNR and contrast (e.g. between white and grey matter) are still maintained by employing lower $F A$ s.

In addition to the experimental results, a summarized analytical comparison with simplified sequence diagrams is shown in Table 3.1. It clearly shows that almost all singleshot techniques suffer from spatial resolution degradation, either blurring or limited as in single-shot turbo-STEAM, HASTE, turbo-FLASH or EPI; all segmented multi-shot techniques frequently experience image distortion (e.g. ghosting) due to phase errors as in multi-shot turbo-STEAM, EPI and GRASE; all techniques involving a series of excitation pulses with high FAs, either for echo refocusing as in FSE and GRASE or for spin excitation as in balanced SSFP GRE, often encounter SAR problem; in addition, susceptibility artifacts appear dramatic in techniques, which either have long TE as in EPI and multi-echo FLASH, or require ideal homogeneity and echo alignment as in balanced SSFP GRE. 


\subsection{Further Improvements for Ultra-fast MRI}

Based on the physical principles and the experimental results, the solution offered by the FLASH technique best suits the prerequisites of signal acquisition for RT-MRI: fast, continuous, with good image quality and without safety problems to patients. Therefore, it is further developed here toward RT-MRI. The general strategies to follow include: 1) reduction of RF pulse duration with slightly increased slice thickness; 2) increase of receiver bandwidth $(B W)$ for elimination of chemical shift artifacts; 3) reduction of $T E$ for minimization of susceptibility artifacts; 4) reduction of $T R$ with lower $F A$ for compensation of signal saturation, and 5) implementation of adaptive $k$-space filter for spike noise reduction.

\subsubsection{RF Pulse Duration, Slice Profile and Partial Volume Effect}

Ideally, a very thin portion of spins with a specific resonance frequency is chosen by the application of an RF excitation pulse in conjunction with a slice-selection gradient. However, finite generation of a single frequency $\omega$ is in practice infeasible. It is necessary to multiply the desired carrier frequency $\omega$ with a compact window function to get a suitable waveform, which corresponds to excitation of a respective slice profile, centered at the position given by Eq 2.5. Because the sinc (i.e. $\sin x / x$ ) pulse has a rect-shaped frequency spectrum and thus produces a rectangular profile which is desired for imaging of an anatomic slice, common waveforms are based on a truncated sinc function with some filtering. Due to the timefrequency relationship, the width of the sinc function $(\Delta T)$ is inversely proportional to the bandwidth of the signal $(\triangle B W)$, i.e. the width of the slice profile $(S L T)$, under a given sliceselection gradient strength:

$\Delta T \propto 1 / \Delta B W$, or $\Delta T \propto 1 / S L T$

so that the duration of the RF excitation pulse $(\Delta T)$ in the time domain is reciprocal to the thickness of the selected slice (SLT) in the spatial domain.

Of course, a reduction of the RF pulse duration leads to a shorter TA. However, a thicker slice may cause unwanted partial volume effect when a large voxel contains a mixture of multiple tissue structures. For most of the dynamic studies employing RT-MRI such as in abdominal or interventional imaging, it is reasonable to have a relatively thicker slice, which also gains better SNR. Therefore, the RF pulse duration is shortened from $2.0 \mathrm{~ms}$ to $0.4 \mathrm{~ms}$, 
which yields a minimal slice thickness of $5.0 \mathrm{~mm}$. In such a way, almost half a second is saved for 256 excitations to acquire one image by the FLASH technique.

\subsubsection{Receiver Bandwidth, SNR and Chemical Shift Artifact}

Shortening of the acquisition time can also be done through increasing the temporal sampling rate of the receiver, which is achieved by increasing the receiver bandwidth $(B W)$ because of the following relation:

$$
\Delta t=1 / B W
$$

where $\Delta t$ is the sampling interval in time, i.e. dwell time, of the receiver. For convenience, the $B W$ is in practice given with a normalized value by the number of acquired samples $\left(N_{s}\right)$ which yields the bandwidth per pixel $(B W / P x)$ :

$$
B W / P x=B W / N_{s}=1 /\left(\Delta t \cdot N_{s}\right)
$$

Because noise is proportional to the square root of the bandwidth, a degradation of the signalto-noise ratio (SNR) is caused by higher bandwidth, i.e. higher sampling rate:

$$
S N R \propto c \cdot \eta \cdot\langle\text { voxel size }\rangle / \sqrt{B W}
$$

where $c$ is a constant factor dependent on the sequence type [111,112] and $\eta$ is a factor dependent on the $k$-space trajectory [112]. For any uniform-density trajectory $\eta$ is one.
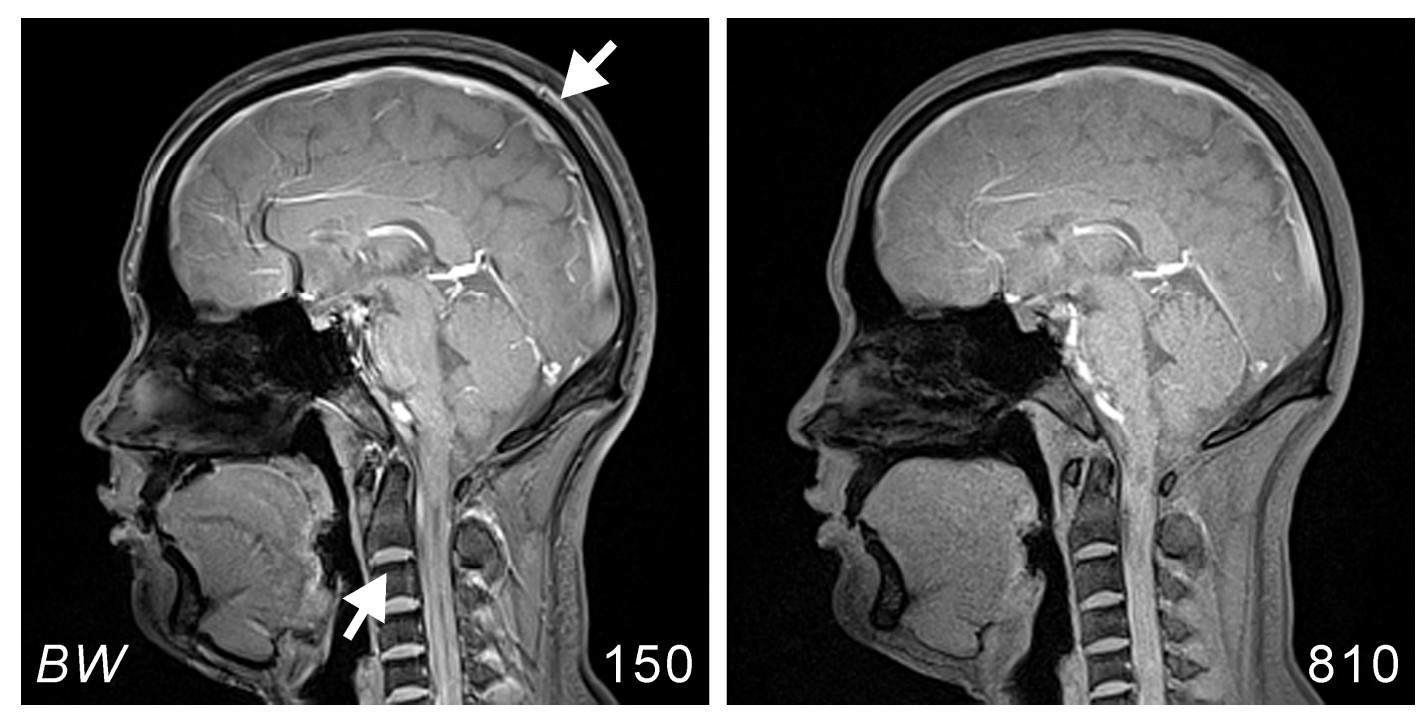

Figure 3.2 Reduction of chemical shift artifacts by increasing the bandwidth $(B W)$ from $150 \mathrm{~Hz} / \mathrm{Pixel}$ to $810 \mathrm{~Hz} /$ Pixel, for an in vivo human brain study (healthy volunteer, male, 28 years old) in a middle sagittal plane. A decrease of SNR is observable, but the appearance of artifacts (white arrow) is much improved. The other imaging parameters: FOV $256 \times 256 \mathrm{~mm}^{2}$, matrix size $256 \times 256$, in-plane resolution $1.0 \times 1.0 \mathrm{~mm}^{2}$, SLT $3 \mathrm{~mm}$, TR/TE 20/4.9 ms, FA $20^{\circ}$. 
However, the variation in precession frequencies of fat and water proton due to their different chemical environment or chemical shift $\delta$, proportional to the main magnetic field strength $B_{0}$ and expressed as parts per million (ppm), may lead to certain pixel shift in the image in frequency-encoding direction:

$$
\text { Pixel Shift }(\mathrm{mm})=\frac{\delta / B_{0}}{B W / P x} \cdot \frac{F O V}{N_{s}}=\frac{\delta \cdot F O V}{B_{0} \cdot B W}
$$

Unlike the dephasing that occurs in magnetic field gradients, the chemical shifts result in separation of the NMR signal into distinct frequency bands. As the receiver bandwidth determines the range of frequencies that must be mapped across a given $\mathrm{FOV}$, a higher $B W$ can effectively eliminate chemical shift artifacts at high field strength like $3 \mathrm{~T}$. This is demonstrated by the above equation as well as in the experiment shown in Fig 3.2, where the artifacts in the frequency-encoding direction (head-foot) was effectively removed by increasing the bandwidth from 150 to $810 \mathrm{~Hz} / \mathrm{Pixel}$.

\subsubsection{Echo Time and Susceptibility Artifacts}

As noted previously, several sources of local magnetic field distortion may result in increased nuclei dephasing, causing an accelerated decay of the transverse magnetization (T2*) and thus a signal loss in GRE images. These include field inhomogeneities, paramagnetic contrast agents, magnetic susceptibility differences such as metal implants or tissue boundaries (e.g. air-tissue interfaces like sinuses, mastoids, bowel) or pathological tissue alterations [113].
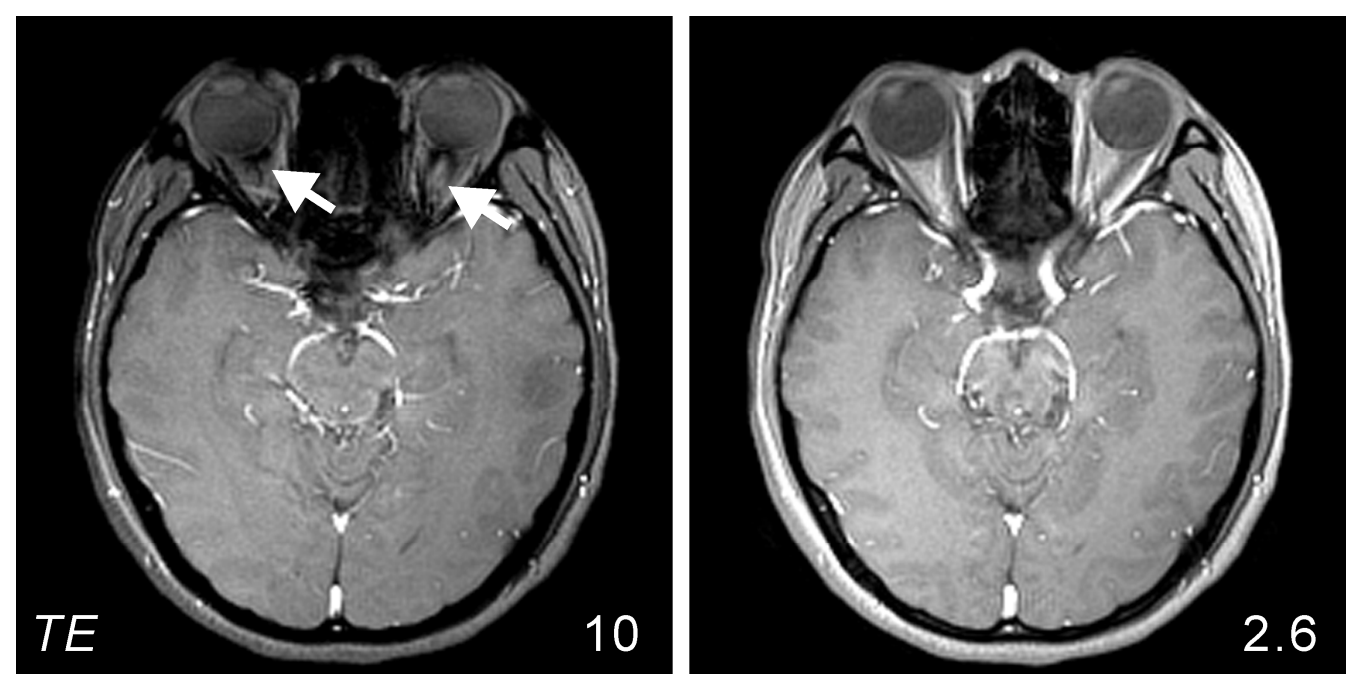

Figure 3.3 Reduction of susceptibility artifacts by decreasing $T E$ from $10.0 \mathrm{~ms}$ to $2.6 \mathrm{~ms}$, for an in vivo human brain study (healthy volunteer, male, 28 years old) in a transversal image plane. White arrows indicate the susceptibility artifacts due to long TEs. The other imaging parameters: FOV $256 \times 256 \mathrm{~mm}^{2}$, matrix size $256 \times 256$, in-plane resolution $1.0 \times 1.0 \mathrm{~mm}^{2}, S L T 3 \mathrm{~mm}, T R 100 \mathrm{~ms}, F A 50^{\circ}, B W 810 \mathrm{~Hz} / \mathrm{Pixel}$. 
As demonstrated in Fig 3.3, the signal loss mainly occurrs around the eyeballs and nasal cavity and builds up over with $T E$. The resulting faintness of the normal tissue structures such as the ocular muscles and the optic nerve (white arrow) impede and diagnosis and functional evaluation. Therefore, the shortest $T E$ is highly demanded to eliminate the susceptibility artifacts or signal void.

\subsubsection{Repetition Time, Flip Angle and General Contrast}

The total acquisition time can be directly shortened by simply reducing the $T R$ of the measurement. However, since such an experiment leads to heavy saturation as described before, the resulting image has very low SNR and contrast, as shown in Fig 3.4 a to d.

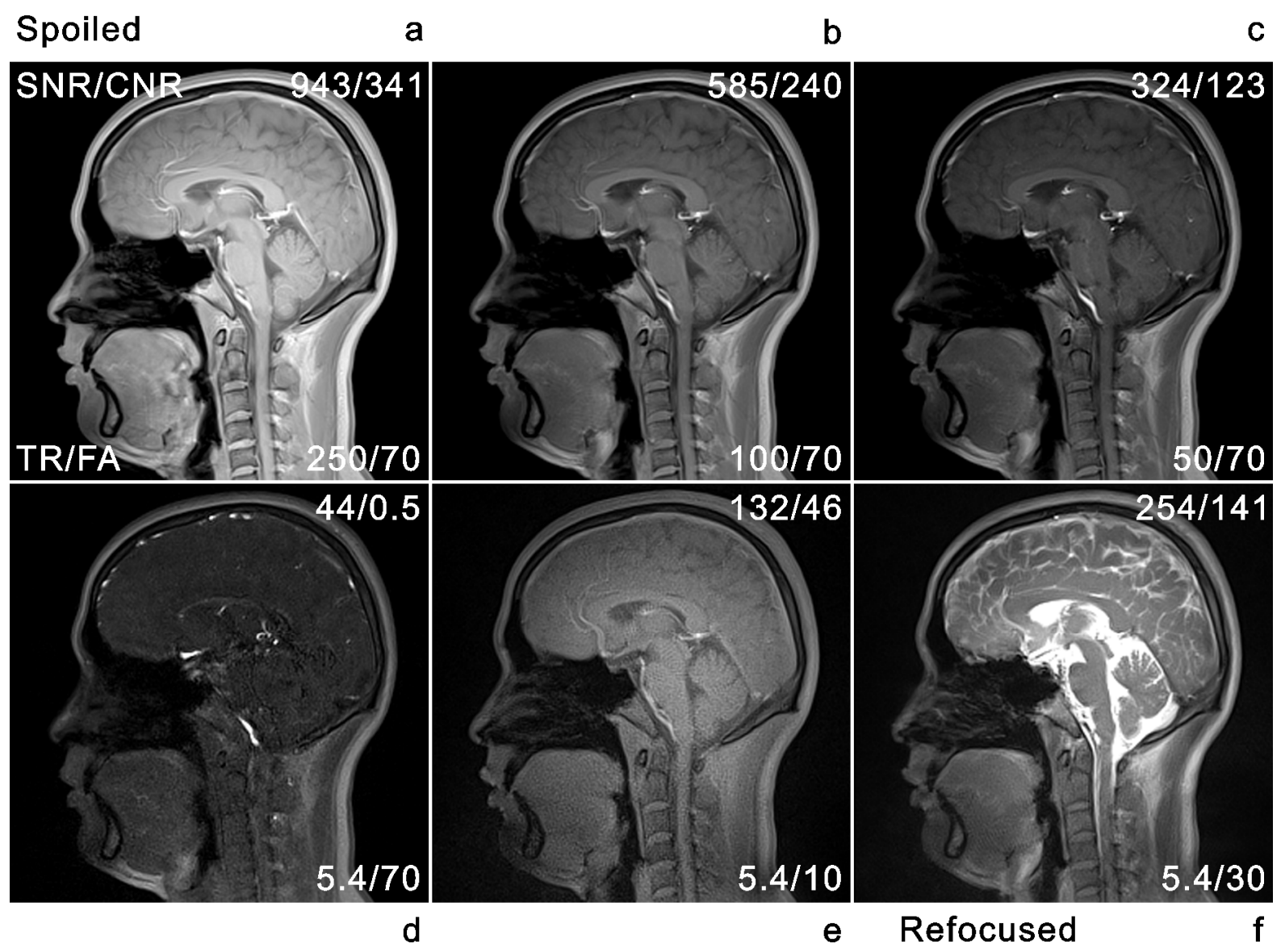

Figure 3.4 Maintaining contrast and signal by lowering $F A$ s at short TRs, for an in vivo human brain study (healthy volunteer, male, 28 years old) in a middle sagittal plane. (a) to (d) show the SNR and CNR as a function of $T R$; (e) shows the effectiveness to maintain certain signal strength and T1 contrast by lowering the $F A$ s from $70^{\circ}$ to $10^{\circ}$; (f) shows a higher achievable contrast related to $\mathrm{T} 1 / \mathrm{T} 2$ by including refocused SSFP signal. (e) and (f) were acquired under optimized conditions with $T R / T E 5.4 / 2.4 \mathrm{~ms}, B W 930$ Hz/Pixel. The other imaging parameters: FOV $256 \times 256 \mathrm{~mm}^{2}$, matrix size $256 \times 256$, in-plane resolution $1.0 \times 1.0 \mathrm{~mm}^{2}$, SLT $5 \mathrm{~mm}$, TA $1.4 \mathrm{~s}$. 
Better results can be achieved by employing smaller FAs to maintain certain SNR and T1weighting [114]. However, sometimes it is still rather difficult, as already stated in the theory [59] and observed from experiment in this thesis, to provide sufficient image contrast, e.g. T1 and T2, which determine the basis of all medical and biological applications of MRI. Therefore, the general contrast insensitivity for extremely short values of $T R$ and $T E$ (TR $\ll$ T1, $\mathrm{TE} \ll \mathrm{T} 2$ ) is compensated by including the transverse magnetization through refocusing gradients and thus by achieving some T2 weighting [115]. These are well demonstrated in Fig 3.4 .

Images in Fig 3.4 a to d show that the image quality is dramatically degraded as the TRs are shortened from $250 \mathrm{~ms}$ to $5.4 \mathrm{~ms}$. Fig $3.4 \mathrm{e}$ shows that certain signal strength and T1 contrast is effectively maintained by lowering the $F A$ s from $70^{\circ}$ to $10^{\circ}$. Fig $3.4 \mathrm{f}$ shows a higher achievable contrast related to T1/T2 by including refocused SSFP signal. The ROI for SNR calculation (about $100 \mathrm{~mm}^{2}$ ) was chosen from the splenium of corpus callosum, and together with the selected ROI from cerebrospinal fluids, the CNR was calculated. The images in e and $\mathrm{f}$ were acquired under the aforementioned optimized conditions: high $B W$, shortest $T R$ and $T E$, low $F A$. The total acquisition time in such a case to yield an artifact-free image with high resolution (e.g. $1.0 \times 1.0 \mathrm{~mm}^{2}$ ) was shortened to about $1 \mathrm{~s}$.

\subsubsection{Spike Noise Reduction}

With much shortened $T R$ to decrease image scan time, the rise time for the gradient amplifier to reach its maximum output value is dramatically decreased. This leads to greater mechanical stress on the gradient coil assembly and increased vibration, which may result in increased random spike noise [116-118]. Such spikes in the raw data (i.e. $k$-space data in the spatialfrequency domain) may generate striation artifacts in the final image [118-121]. Since experiments have shown that a proper hardening of the coil assembly or the securing of loose cables cannot correct such noise, a more effective method is required during the acquisition and before image reconstruction.

Although a number of techniques have already been proposed [116, 117, 122-127] for searching and correcting spikes, many of them are unsuitable due to either the requirement of specific hardware [123] or the infeasibility for online processing [124, 125, 127]. Therefore, a simple strategy based on thresholding is developed in this thesis. Compared to the previous techniques, the current one does not involve any averaging [117, 122], which avoids the 
artificial inclusion of corrupted neighboring data points, or masking [116] and modeling [126], which eliminates computation burden and prolonging of the processing time.

The implemented method depends mainly on two scale factors. The first parameter $r$ defines a radius from the $k$-space center and the second parameter $A$ is a fraction of the maximum amplitude $A_{0}$ along each radial line of $k$-space across the center.

The spikes have typically very high magnitude, sometimes even higher than that of the echo center region, as shown in Fig 3.5 (black arrow heads). The striation artifacts come mainly from those spikes located in the $k$-space periphery where the magnitude is relatively low. Therefore, as the first step, the thresholding strategy searches for spikes located outside the radius $r$ from the echo center $r_{0}$, while all data within this radius are ignored to avoid eliminating true data from the echo peak. This is similar to applying specific thresholding over a low-pass filter, and such filtering in a radial direction is especially advantageous when applying radial $k$-space encoding rather than rectilinear Cartesian encoding. The magnitude of a spike usually has also very sharp character besides its abnormal height compared to the surrounding high frequency signals with low strength. Therefore, any data point is regarded as a spike if its magnitude $A$ meets two conditions, which rely on the magnitude value of the complex $k$-space data: larger than $1 / a$ of the maximum magnitude value along that radial line (i.e. $A_{r}>A_{0} / a$ ), and at the same time also larger than $1 / a$ of either of the magnitude value of its two neighboring data points (i.e. $A_{r}>A_{r \pm 1} / a$ ) to make sure that it is truly a spike rather than large signal fluctuation. In the current implementation $r$ is chosen to be $1 / 4$ of sampling points $\left(r=N_{S} / 4\right)$ and $A$ is set to be 1/10 of the maximum magnitude value along the radial line $(a=10)$, both of which are determined empirically from several trial experiments so as to be satisfactory in minimizing false negative and false positive errors. Since the maximal magnitude is determined from the raw data, the threshold value that depends on it becomes adaptive to each coil element, or to each signal acquisition (readout) if in radial encoding scheme. To find this real maximum of the magnitude data in each radial line and avoid locating a local minimum, it is determined among the center 16 data points, i.e. $r_{0} \pm 8$, where $r_{0}$ is the echo center at $N_{s} / 2$.

As the second step, once a noise spike pixel is encountered, the real and imaginary parts of that data point are replaced by zero. After thresholding and replacement of spike noise data points, the resulting raw data set is processed further in the usual way to form the final image. For demonstration purposes, the current thresholding method is implemented in the MRISim software [128]. But it should be noticed that, it can be easily implemented in an online processing work frame. 

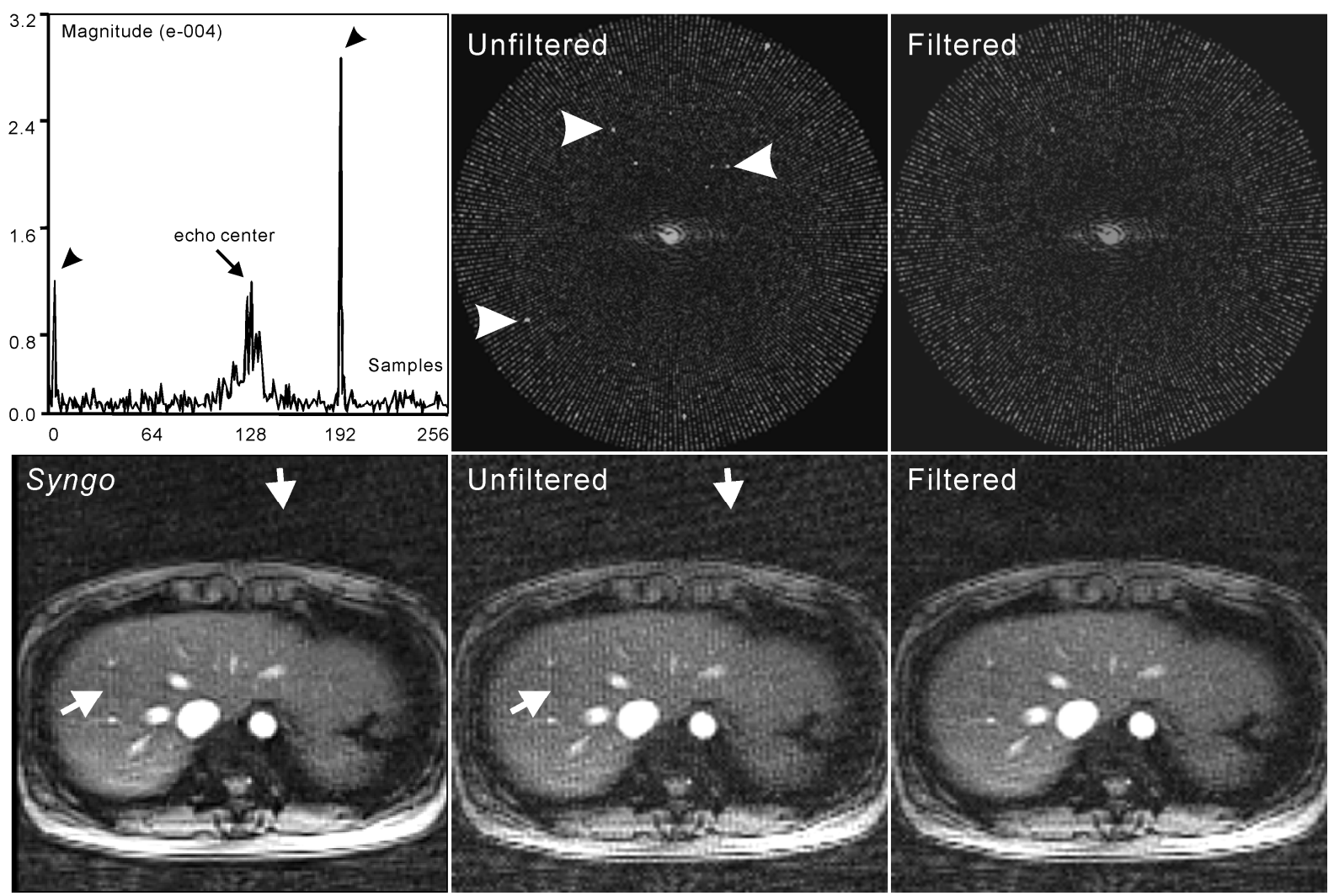

Figure 3.5 Spike noise reduction and removal of striation artifacts by an adaptive $k$-space filter based on a thresholding strategy. Data were acquired from a radial FLASH RT-MRI experiment for an in vivo human abdominal study (healthy volunteer, female, 27 years old) in a transversal plane across the liver, and were reconstructed in MRISim [128]. Upper left: one encoding line of the $k$-space with two spikes on both sides of the echo center; Upper middle and upper right: the unfiltered and filtered $k$-space data of one image, respectively; Lower middle and lower right: the corresponding images with and without filtering after reconstruction; Lower left: the image reconstructed directly from the manufacturer's software (Syngo) with an applied Gaussian low-pass filter. Arrow heads: spike noises in $k$-space; white arrows: striation artifacts in the reconstructed images. The imaging parameters: spoiled radial FLASH, 16-channel body matrix coil (Siemens AG, Erlangen, Germany), FOV $256 \times 256 \mathrm{~mm}^{2}$, matrix size $128 \times 128$, in-plane resolution $2.0 \times 2.0$ $\mathrm{mm}^{2}$, SLT $8 \mathrm{~mm}$, TR/TE 2.02/1.30 ms, FA $8^{\circ}, B W 1950 \mathrm{~Hz} / \mathrm{Pixel}$.

One example of applying the adaptive $k$-space filter on the actual measured data from an in vivo abdominal RT-MRI experiment is presented in Fig 3.5. A large number of such spikes were present in the whole $k$-space of one chosen time frame (white arrow heads), while the striation artifact patterns were obvious in the corresponding reconstructed image in both liver and background (white arrows). After applying the proposed filtering strategy, the striation artifacts at these two places were successfully removed. Only a few spikes remained in the filtered $k$-space which was missed by the thresholding strategy. Nevertheless, considerable improvements of the artifact reduction were visible in the final image. Noticeably, the pure Gaussian low-pass filter offered by the software from the manufacturer (Syngo) had the similar effect but not efficient enough as compared to the implemented strategy. 


\subsection{Summary}

The period from the early 1980s to the late 1990s has seen an explosive growth in the range and power of high speed MR imaging techniques with rapid pulse sequences [129-133]. The corresponding acquisition times for a complete MR image vary from sub-seconds (e.g. EPI) to several minutes (e.g. FSE). The enormous gain in shortening of imaging times not only improved patient comfort and clinical turnover, but also allowed for imaging in three dimensions or minimized patient motion artifacts in two dimensions. For example, "filming" of the heart with ECG synchronization and within a single breath-hold became feasible [62, $68,81,100,101]$.

Although diverse, all techniques are based on spin echoes, stimulated echoes, gradient echoes or a hybrid type. They are also related in that the shortened acquisition time is achieved by a compromise, i.e. a certain penalty, in image quality, either in spatial resolution, SNR, image contrast or artifacts.

Therefore, a proper choice of the signal acquisition technique and imaging pulse sequence is of crucial importance for the development of RT-MRI. As a physical principle, the basic prerequisite is speed, continuity, image quality, and safety to patients. Through a detailed theoretical and experimental comparison the FLASH technique, which generates fast gradient echo signals with steady state precession acquisition, is chosen and further exploited. Its advantage is the ability to shorten the $T R$ in concert with a low $F A$ to maintain adequate image SNR, while still achieving ultra-fast imaging. In addition, and even more important advantage is that, the measuring time is not limited by physical properties such as the duration of a relaxation time. Thus, an unrestricted and case-dependent choice of spatial and temporal resolution, i.e. a flexible trade-off between speed and image resolution, can be achieved. Under such conditions, the total acquisition time for an artifact-free image with high spatial resolution was shortened to about $1 \mathrm{~s}$, which is appropriate for real-time imaging. Further improvements in spatial encoding and corresponding reconstruction are described in the next chapters. 


\section{Chapter 4}

\section{Development of Real-time MRI: $k$-Space Encoding}

From a physics point of view, the available magnetization may be efficiently utilized in the FLASH technique with low $F A$ s and very short $T R$ s. The remaining question from an image formation point of view is how to efficiently deal with the acquired data, i.e. encode the signal samples in the $k$-space. This is because the resulting image quality depends on how the $k$ space is filled. For example, the spatial resolution in an MR image is limited by the range of $k$-values covered, while the sampling density within this range determines the image size, i.e. FOV, which should be depicted without aliasing.

One attempt is to travel through $k$-space as fast as possible for every repetition (or readout), which corresponds to switching the gradient with a slew rate and strength as high as possible. Unfortunately, this is restricted both in technique and in biology, because the available gradient performance is determined by real amplifiers and thus limited in amplitude and in how quickly it can change, while excessive gradient switching interferes with neural electrophysiology in biological systems and causes peripheral nerve stimulation (PNS). Therefore, ever-faster encoding cannot be pursued much further with such a concept. The other attempt is to determine how to travel through the $k$-space with a proper choice of specific trajectories, i.e. encoding patterns. According to Eq 2.11, with appropriate $G(t)$, a particular trajectory $k(t)$, along which the sampled signal is stored, can be selected to cover the $k$-space. After $S(k)$ is adequately sampled (Eq 2.12), it is transformed by applying desired reconstruction algorithm to obtain an image. Such determination of how the acquired signal is mapped into the $k$-space has thus a dramatic effect on the spatial and temporal resolution, as well as the contrast of the resulting images and the scan duration.

In this chapter, the advantages of a radial encoding scheme are demonstrated, whereas the problems of conventional Cartesian encoding are explained. In addition, specific approaches, namely sampling pattern optimization and trajectory correction, are implemented for the development of RT-MRI. 


\subsection{Spatial Encoding with Radial Trajectories}

In a radial encoding scheme, the $k$-space signal is sampled along spokes - rotated lines instead of parallel lines as in a Cartesian scheme. This can be done by applying gradients in the $\mathrm{x}$ - and $\mathrm{y}$-direction with various amplitudes:

$$
G_{x}=G \cdot \cos \emptyset, G_{y}=G \cdot \sin \emptyset
$$

where $\varnothing$ is the desired angle of the spoke and $G$ denotes the constant strength required for sampling the central $k$-space line, which can be calculated based on Eq 1.11:

$$
G=\frac{B W}{\gamma \cdot F O V}
$$
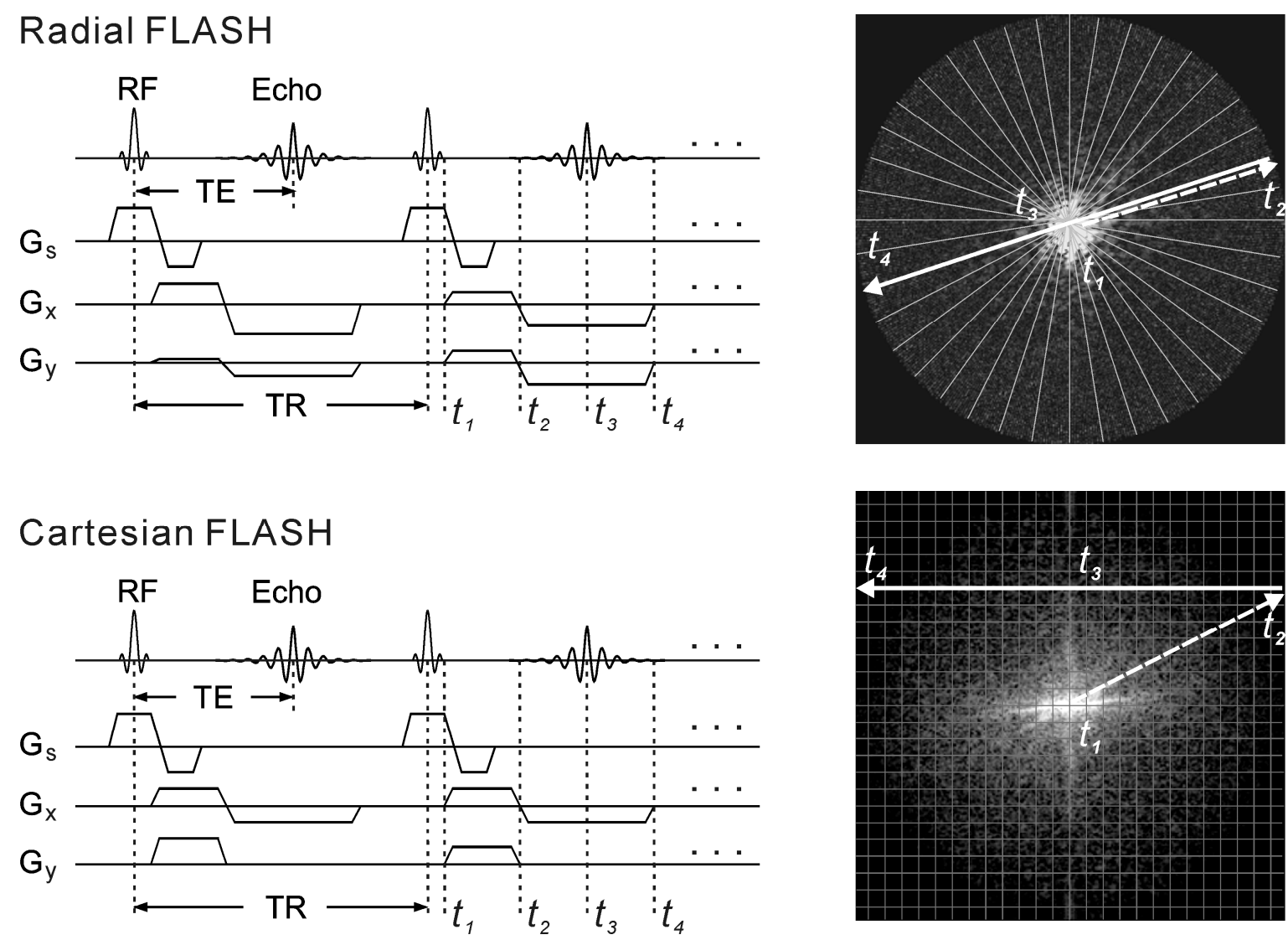

Figure 4.1 Sequence diagrams (left) and $k$-space trajectories (right) for radial and Cartesian FLASH.

Fig 4.1 illustrates the gradient waveforms of the spoiled FLASH technique using radial and Cartesian encoding schemes. The corresponding $k$-space trajectories with one signal readout (encoding line) are also shown on the right side. The solid lines and arrows indicate the $k$ space sampling trajectory of the sequence, while the dashed lines indicate the trajectory before the sampling. More details of each gradient are explained in the pulse sequence design part of Chapter 6. 
Radial encoding was already proposed in 1973 as projection reconstruction (PR) imaging when the first spatially resolved MR image was presented [6]. But it was soon replaced by Cartesian encoding because the latter partially compensates for the off-resonance effects and technical inadequacies in gradient performance in the early MRI systems. In recent years radial encoding is regaining growing interest [29], mainly because of several unique advantages relative to Cartesian encoding.

\subsubsection{Undersampling beyond the Nyquist Limit}

First of all, radial spokes and Cartesian lines are both literarily called "views", but the view number in radial scheme is independent from the data matrix size, i.e. image resolution. This offers flexibility in choosing the repetition steps for acquiring a whole image, and thus the possibility of undersampling.

Normally, in order to make sure that the maximum distance between two samples in the $k$-space $(\Delta k)$ does not exceed the Nyquist limit, Eq 2.14 has to be fulfilled for radial encoding in the readout direction $k$ :

$$
\Delta k=\frac{2 k_{\max }}{N_{S}} \leq \frac{1}{F O V}
$$

and in the azimuthal direction $\emptyset$, i.e. two farmost samples at the edge of the $k$-space:

$$
k_{\max } \cdot \Delta \emptyset=k_{\max } \cdot \frac{\pi}{N_{V}} \leq \frac{1}{F O V}
$$

By combining the above two equations, the number of views that have to be acquired in order to fulfill the Nyquist criterion for a radial image with the same resolution as that for a Cartesian image with square FOV, i.e. $N_{s}=N_{P E}$, yields:

$$
N_{V} \geq \frac{\pi}{2} \cdot N_{P E}
$$

Therefore, to obtain an image with resolution of $N_{P E} \times N_{P E}$, more repetitions (about $1.57 N_{P E}$ ) are required in radial scheme if the sampling rule is to be obeyed. However, different to omitting the number of parallel sampled lines in Cartesian scheme, which corresponds to periodic replication of the point spread function (PSF) and wrap-around object in the image as described before, reducing the view number in radial encoding scheme results in broadening of the PSF main lobe and increasing of the side lobe amplitudes, which corresponds to a decrease of the effective FOV, i.e. artifact-free area in the middle of the image around the PSF [28]. Undersampling artifacts appear as streaks in the image, but at the edge [27] while the main structure of the object is maintained. Thus, in practice, the number of repetitions can be 
greatly reduced and the scan duration can therefore be shortened. This is well demonstrated in the next section with experimental results.

Acquiring views below the Nyquist limit introduces "Nyquist holes", i.e. unmeasured regions in $k$-space, which affect the weighting function and result in deviation of the modulation transfer function in the gridding reconstruction (see section 5.1). The failure of the reconstruction algorithm then causes predominant streaking in the vicinity of the object in the image (Fig 4.3). To control the degree of artifact level and number of view $N_{V}$ [134, 135], a moderate undersampling with the number of views close to the image base resolution has always been chosen for real-time applications in this thesis.

\subsubsection{Free Zooming without Aliasing}

The ability to perform readout oversampling in all directions with rotated views, in contrast to only one direction in Cartesian scheme, allows for increasing the FOV in radial encoding, which shifts away the periodic replications of the object due to discrete sampling. In this case, the gradients remain unchanged, while the sampling rate of the receiver is doubled, so that the spatial resolution is preserved but the apparent FOV is actually doubled (according to Eq 3.1). This can further facilitate selecting a smaller FOV than the actual object size without any signal aliasing or additional acquisition steps - like zooming into a local area, which is potentially useful during interventional or interactive imaging.

\subsubsection{Intrinsic Robustness against Motion}

Another advantage of radial sampling is that, due to the absence of phase encoding, motioninduced phase errors are greatly reduced, which eliminates the troublesome ghosting artifacts as frequently shown in Cartesian imaging [29, 136, 137]. Moreover, the intrinsic oversampling of center $k$-space also contributes to reduce motion artifacts in a manner similar to signal averaging [138]. This is at the expense of mild smearing or blurring, and in worst cases as streaks due to motion inconsistencies. Noticeably, while in Cartesian encoding the ghosting artifacts appear over the entire image with maximum intensity occurring at the lowest order ghosts closest to the object, in radial encoding the streaking artifacts occur perpendicular to the motion direction with lower intensity closer to the object. Typical results are shown in the next section where the potential capability for studying dynamic process is 
well demonstrated.

Moreover, in contrast to Cartesian scheme, each sampled view in radial encoding captures an equal amount of low and high spatial frequency information in the $k$-space, offering more homogeneous image updates in dynamic MRI applications, which is to be discussed in details in the next chapter.

\subsection{Comparison of Available Encoding Schemes}

Since many different trajectories can be used to sample the $k$-space, a few other major schemes are discussed here. Moreover, a summarized comparison among radial, Cartesian, and spiral encodings is given based on simulated data, particularly in terms of their artifact properties due to undersampling, off-resonance and motion. At the same time, detailed experimental comparisons are also presented to demonstrate the advantages of the radial encoding scheme.

\subsubsection{Brief Review}

\section{Cartesian}

Cartesian is the most often used way of $k$-space sampling. It encodes the acquired data in the $k$-space in a line-by-line scheme [50]. For many years, Cartesian $k$-space encoding has been the most popular method because it allows for a direct use of the inverse FFT. As all sampled data lie directly on a rectilinear grid which forms the raw data matrix, by performing FFT for each line and each column subsequently, an image of the object with identical extent can be obtained. However, such a rectilinear incremental phase encoding scheme has many drawbacks, especially when aiming at imaging time acceleration or imaging of dynamic processes.

First of all, as described in Section 2.2.3, any reduction of the number of the encoding steps to shorten acquisition time may result in either decrease of image spatial resolution or aliasing of signal in the phase encoding direction, as both illustrated in Fig 4.3 by performing 2-fold undersampling from 256 to 128 with different strategies. Typically, these artifacts may also occur when the selected FOV is smaller than the actual object size. This is because the signal originating from the anatomy outside the FOV is mis-mapped into the FOV, i.e. 
assigned with a phase value that has already been given to signal originating within the FOV.

Since frequency encoding is normally carried out according to the Nyquist theorem with a double rate of digitizing, i.e. readout oversampling by a factor of two, such aliasing effect is only critical in the phase encoding direction. To avoid such an effect, oversampling in the phase-encoding direction is necessary and the resulting extended portion of the FOV is discarded during reconstruction, while only the selected one without artifacts is displayed. However, this is at the cost of the increased imaging time.

The second disadvantage is that any motion that occurs during scanning may produce replications of the moving object across the image, i.e. ghosting artifacts, in the phase encoding direction [139]. This is because the position change along this direction during the signal acquisition causes misplacement of different phase values in the $k$-space assigned by the phase-encoding gradient with different amplitude from one $T R$ to another, which in turn is converted by the Fourier transformation into the image. Although experiments in this thesis showed that with optimized pulse sequence parameters as described in Chapter 3 (i.e. shortest $T R$ and $T E$, low $F A$, with high $B W$ ), Cartesian FLASH could eliminate severe ghosting during, for example, periodic respiration in abdominal imaging or pulsatile motion of vessels in carotid imaging, due to substantially shortened acquisition window and decreased intra-line phase inconsistency, it still suffers from minor ghosting and blurring during imaging of irregular or abrupt motions, e.g. displacement of temporomandibular joint (TMJ) during jaw opening or swallowing, as illustrated in Fig 4.5.

Furthermore, individual lines are not equivalent as they encode either low or high spatial frequencies (in the phase encoding direction). This leads to unwanted consequences for the update of Cartesian images when studying moving objects, which will be described in details in the next chapter.

\section{Spiral}

Spiral $[8,140-143]$ is another commonly used non-Cartesian encoding scheme besides radial. The trajectory starts from the $k$-space center and ends in the edge area by using the pulse sequence with two simultaneously oscillating gradients $G_{x}$ and $G_{y}$. The advantage of spiral encoding is that the center of the $k$-space is sampled immediately after the RF excitation when very little dephasing has occurred, and the $k$-space trajectory is smoother than EPI and do not require ultrafast gradient switching. The disadvantage of spiral includes its high sensitivity to off-resonance effects, as significant blurring shown in Fig 4.2, and also the limitation of the 
resolution due to $\mathrm{T} 2 *$ relaxation when using a single-shot sequence or longer scan time and more artifacts when using a multi-shot interleaved variation [144, 145]. Although spiral encoding is proved to be less sensitive to flow artifacts due to intrinsic compensation of higher gradient moments [138, 143, 146], it remains sensitive to object motion, particularly when compared to radial encoding [147]. More importantly, undersampling in spiral scheme may lead to coherent artifacts throughout the image, unlike the benign undersampled artifacts in radial scheme. Similarly, more complicated fold-in patterns rather than simple discrete aliasing as in Cartesian case may be invoked in spiral sampling, when the FOV is selected smaller than the imaging object. All these characteristics were demonstrated in Fig 4.2.

\section{PROPELLER}

PROPELLER (periodically rotated overlapping parallel lines with enhanced reconstruction) [148-151] is a hybrid encoding scheme combining radial and Cartesian sampling. The trajectory is made up of a set of parallel Cartesian lines, termed as blades, which are rotated to cover the entire $k$-space. Various pulse sequences can be used to acquire these Cartesian lines, such as EPI [150, 152], FSE or FLASH. Because each blade runs through the central $k$-space and can be reconstructed separately to obtain a low-resolution image, the advantage of motion artifact correction by examining this information has been demonstrated in various studies [153-156]. Although this technique is already commercially available on most MRI systems, it is excluded in this work because of its remarkable longer imaging time compared to normal Cartesian and radial schemes, due to strong oversampling of the central $k$-space area, and because of its complicated reconstruction. More importantly, it has the difficulty to combine with sliding window technique to increase image reconstruction rate.

\section{EPI}

Echo-planar imaging (EPI) is normally also regarded as a special type of encoding scheme, because repetitive alternations of the readout gradient polarity is continuously applied during the whole imaging process. It is frequently used for fast acquisition and encoding in clinical routine examinations and research as well. Generally it covers the entire $k$-space in either a zigzag (with constant or non-blipped phase encoding gradient) or rectilinear (with intermittent or blipped phase encoding gradient) global trajectory after one single RF excitation. However, because of a number of related problems including limited spatial resolution due to $\mathrm{T} 2 *$ decay, predominant artifacts such as geometric distortion and signal loss caused by phase errors that 
Trajectory

Undersampling

Zooming

Motion
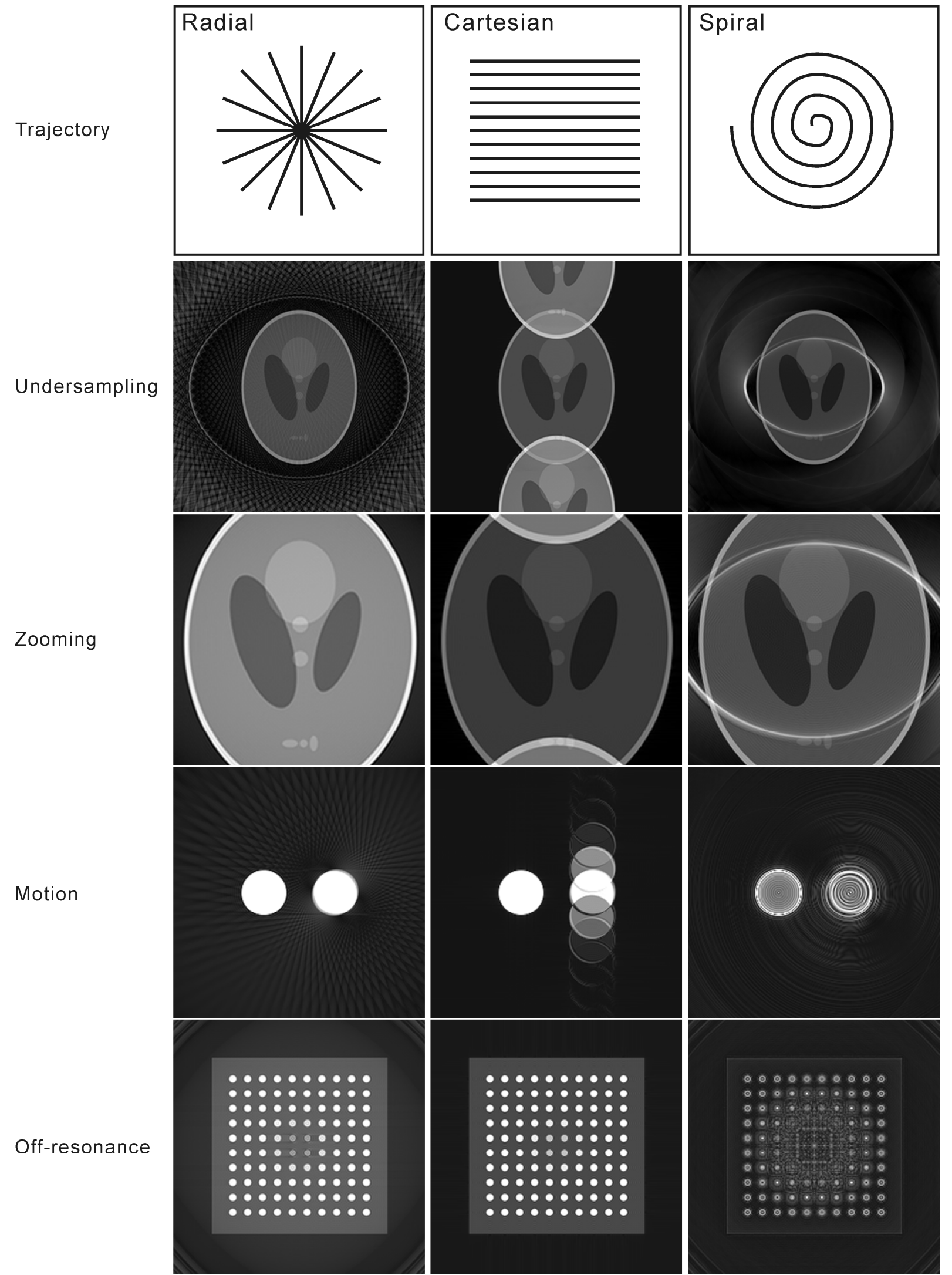

Figure 4.2 Comparison of radial, Cartesian and spiral encoding schemes. The $k$-space trajectories are given on top, and corresponding artifacts due to undersampling, zooming, motion and off-resonance are shown for simulated data using a numerical phantom (MRISim software [128]). 
are accumulated due to off-resonance effect $[157,158]$ or ghosting artifacts due to alternated gradient, as described and demonstrated in Section 3.2, it is also excluded here for further discussion.

In addition, many other 2D $k$-space trajectories also exist, such as Lissajou [159-161], circular [162, 163], linogram [164, 165], TWIRL (twisted radial lines) [166, 167], stochastic [168], rosette [169], WHIRL (winded hybrid interleaved radial lines) [170], and STAR [171]. Most of them are derivatives or combinations of the basic non-Cartesian trajectories, i.e. radial and spiral, and try to compensate for the drawbacks such as sensitivity to the field inhomogeneities and susceptibility differences. However, due to their complex sampling patterns and resulting difficulties in practical implementation as well as in image reconstruction, most of them are applied only in very limited cases. Therefore, this comparison focuses mainly on Cartesian, spiral and radial encoding schemes.

Simulated data with numerical phantoms to demonstrate different artifact produced by, undersampling, zooming, motion, and off-resonance, are presented in Fig 4.2. As discussed above, radial encoding has more incoherent undersampling artifacts as streaks with an artifact-free area in the middle of the image, compared to Cartesian and spiral encoding. And, it is obvious that the "zooming" ability without prolongation of the imaging time is a unique property of radial trajectories while the other two result in in-folding artifacts similar to the undersampling situation. For objects with motion during the signal acquisition, the induced streaks around the object with highest intensity at the image edge in radial encoding scheme are much more benign when compared to the overall aliased ghosts in Cartesian or complicated artifact pattern in spiral encoding. Although compared to smearing and signal loss in radial encoding, Cartesian scheme hardly shows visible artifacts due to off-resonance effects, by considering the general characteristics, it is still concluded that the advantages of employing radial encoding scheme outweigh the qualities obtained from either Cartesian or spiral trajectories, particularly to serve as an efficient mean for dynamic imaging.

\subsubsection{Experimental Comparison}

A thorough study of the undersampling artifacts from an in vivo measurement with human brain (healthy volunteer, female, 37 years old) is presented in Fig 4.3. The Cartesian encoded images are shown from a to $\mathrm{d}$ with the corresponding $k$-spaces below them, whereas the radially encoded images are shown from e to $\mathrm{h}$ with also corresponding $k$-spaces. For better 


\section{Cartesian}
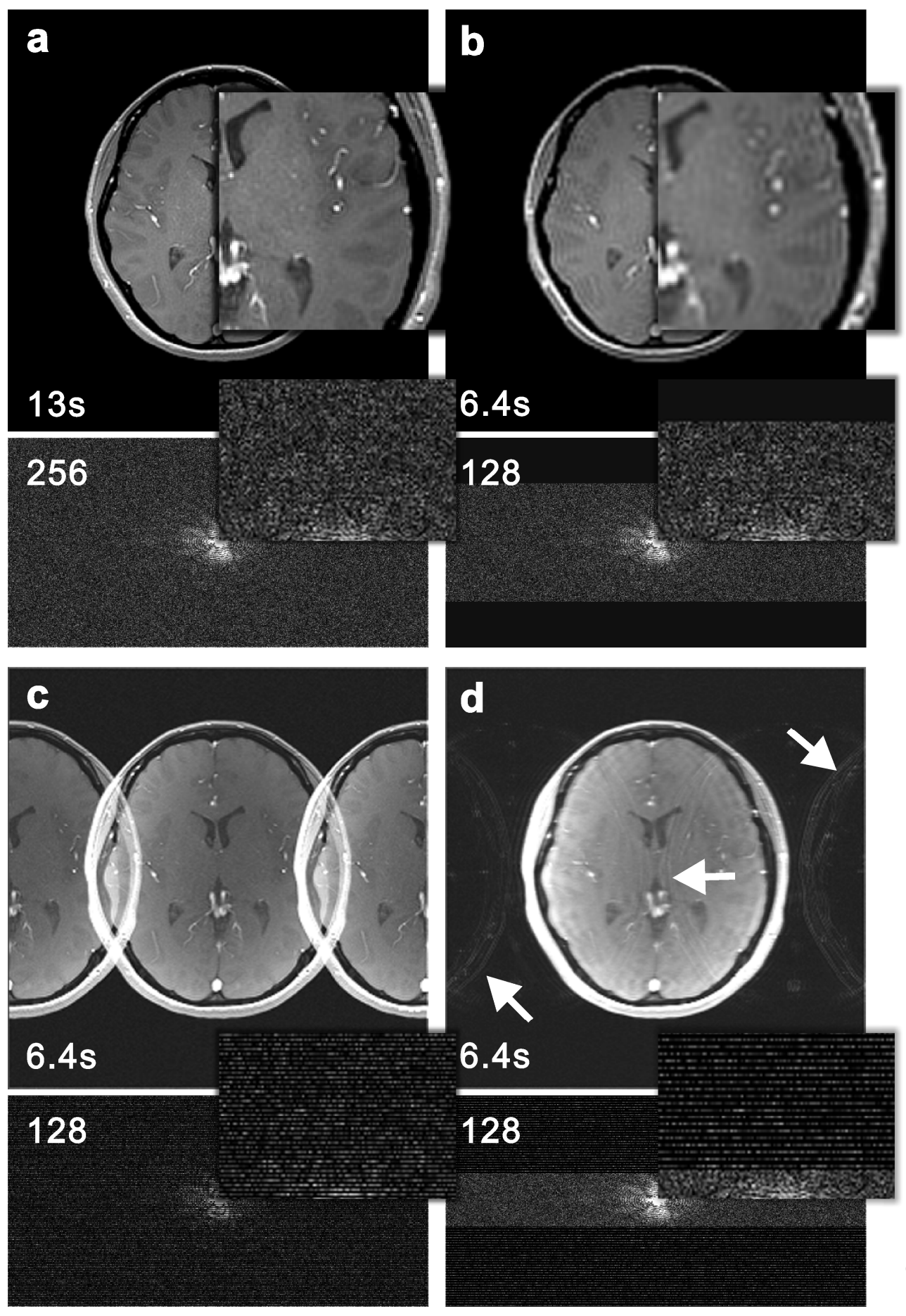

Continued

Figure 4.3 Undersampling effect of (a to d) Cartesian and (e to h) radial encoding, with magnified view of detailed part for an in vivo human brain study. Both images and the corresponding $k$-spaces are illustrated. The number of the phase-encoding lines (Cartesian) and views (radial), as well as the TA and SNR, are given in the figure. In Cartesian, oversampling in the frequency-encoding direction (left-right) resulted in a rectangular raw data matrix. Other parameters were identical for both encoding schemes: spoiled FLASH, FOV 256×256 mm², SLT $3 \mathrm{~mm}$, TR/TE 50/2.7 ms, FA 30 ${ }^{\circ}, B W 430 \mathrm{~Hz} / \mathrm{Pixel}$. 
Radial
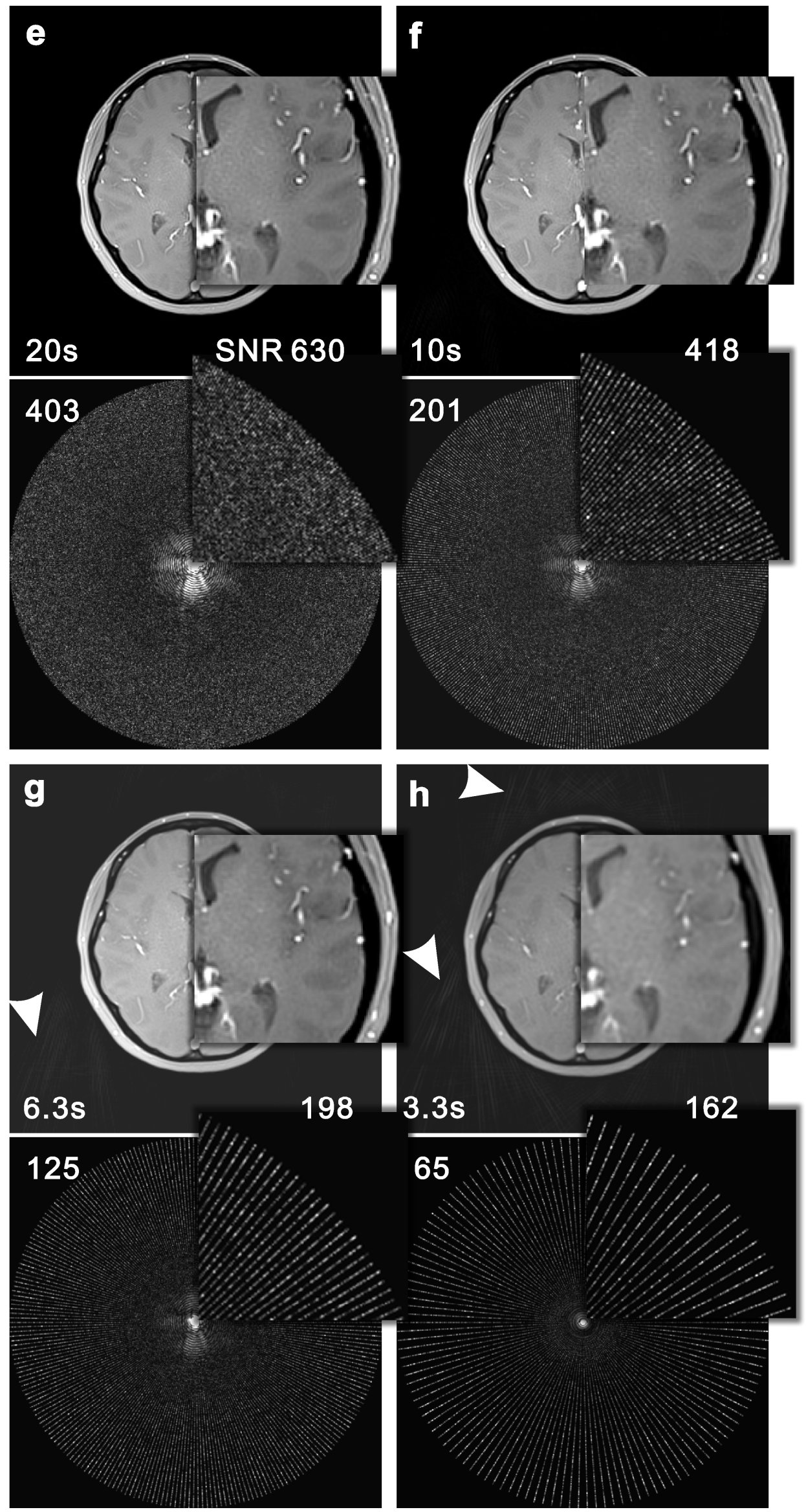
demonstration, the detailed parts are magnified. Fig $4.3 \mathrm{a}$ and d shown images with full sampling for a FOV of $256 \times 256 \mathrm{~mm}^{2}$ and a matrix size of $256 \times 256$ in both cases -256 phaseencoding lines in Cartesian and 403 views in radial encoding, which yield the same in-plane spatial resolution of $1.0 \times 1.0 \mathrm{~mm}^{2}$.

In Fig 4.3b, 2-fold undersampling with 128 phase-encoding steps in Cartesian by keeping the same line space resulted in half of the $k$-space size and thus lower spatial resolution $\left(2.0 \times 2.0 \mathrm{~mm}^{2}\right)$. Whereas in Fig 4.3c, same undersampling but keeping the $k$-space size resulted in double line space and thus aliasing artifacts and blurring in the image. Fig $4.3 \mathrm{~d}$ was also 2-fold undersampled, but in this case the central $k$-space are fully sampled with 64 lines (i.e. same line space as that in a and b) and the periphery was 1.5 -fold undersampled with the rest 64 lines (i.e. same line space as that in c). With such a variable density undersampling scheme, the resulting aliasing was much weaker (arrows) because the $k$-space center, which contains most of the signal and contributes more to image contrast was not undersampled. However, the artifacts are still difficult to remove. For radial encoding, reducing the views from full sampling with 403 views to 6-fold undersampling with only 65 views (Fig 4.3h), while keeping the image matrix size $(256 \times 256)$ constant resulted in blurring, decreased SNR and mild streaks (arrow heads) closer to the image object. However, the spatial resolution and the main structure were maintained without signal aliasing. The $T A$ is also given in the figures, which clearly shows that much shortened imaging time could be achieved in radial encoding by simply reducing the number of views due to its benign undersampling behavior.

Similarly, results from the zooming study in both encoding schemes are presented in Fig 4.4. Fig $4.4 \mathrm{a}$ shows the original image with a FOV of $256 \times 256 \mathrm{~mm}^{2}$ and a matrix size of $256 \times 256$, which yielded an in-plane resolution of $1.0 \times 1.0 \mathrm{~mm}^{2}$. Fig $4.4 \mathrm{~b}, \mathrm{c}$ and $\mathrm{d}$ show the "zoomed-in" images corresponding to the white square in a, with a smaller FOV of $64 \times 64$ $\mathrm{mm}^{2}$. To obtain the same spatial resolution, 64 phase-encoding steps were acquired in Fig $4.4 \mathrm{~b}$ using Cartesian encoding. The signal from outside of the FOV is folded into the chosen FOV in the phase-encoding direction, i.e. anterior (top in the image) to posterior (bottom in the image), and thus distort the image. As one of the remedy, Cartesian encoding with 2-fold oversampling in the phase-encoding direction with the same FOV and matrix size was done, resulting in an artifact-free image as shown in Fig $4.4 \mathrm{c}$ but at the cost of doubling the measurement time compared to Fig 4.4b. In contrast, by applying radial encoding with the same matrix size and relevant view number (i.e. 65), a clear image with the same measurement time as compared to Fig $4.4 \mathrm{~b}$ could be obtained due to oversampling in all 
radial directions. The image experienced only mild blurring and decreased SNR due to undersampling. It should be mentioned that, such aliasing artifacts can also be corrected during image reconstruction by exploiting different spatial sensitivity of multi-channel receiver coils, termed as parallel acquisition techniques (PAT). More details will be discussed in Section 5.3, but it should be noticed that, although PAT reduces the amount of data to be acquired and thus the scan time in conventional Fourier encoding, the effectiveness of the algorithms, regarding the removal of the artifacts and the decrease of the SNR, practically limit the reduction factor.
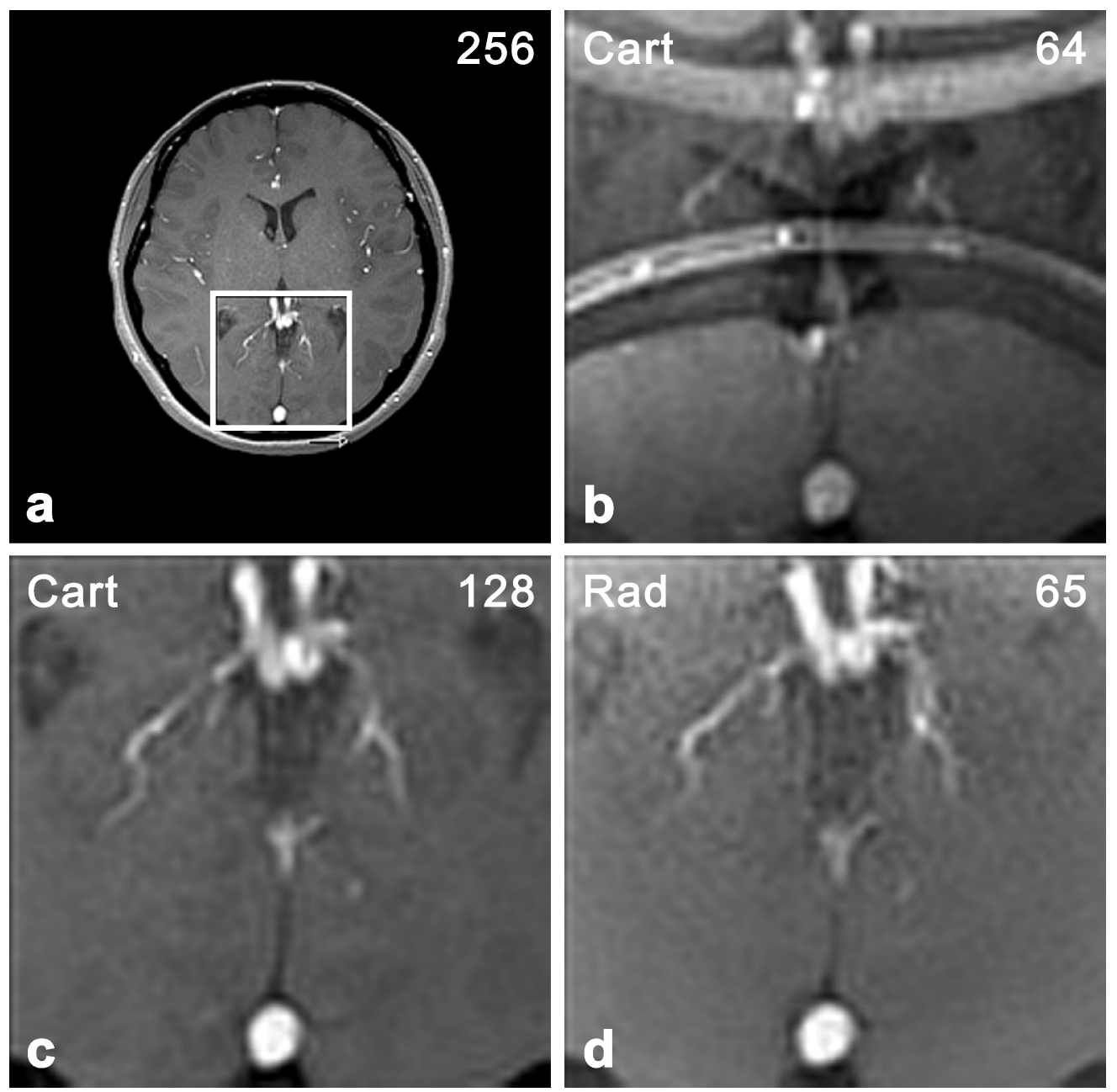

Figure 4.4 Zooming effect of Cartesian and radial encoding for an in vivo human brain study in a transversal plane. (a) FOV $256 \times 256 \mathrm{~mm}^{2}$, matrix size $256 \times 256$, and in-plane resolution $1.0 \times 1.0 \mathrm{~mm}^{2}$. The white square indicates the "zoomed-in" area in (b, c and d). (b) Cartesian encoding - smaller FOV 64×64 $\mathrm{mm}^{2}$ with matrix size of $64 \times 64$, same in-plane resolution as in (a) but with strong aliasing artifacts. (c) Cartesian encoding - same FOV and matrix size as in (b) but 2-fold oversampling in the phase-encoding direction. (d) Radial encoding - same FOV and matrix size as in $\mathbf{c}$ with view number of 65 .

In addition, motion robustness was investigated with two examples shown in Fig 4.5. The displacement of temporomandibular joint (TMJ) during mouth opening is an irregular movement because both in-plane inferior and anterior displacement as well as through-plane 
medial displacement of the joint condyle (indicated by $c$ in the figure) occur during this period. The motion of oropharyngeal organs during swallowing is an abrupt movement because several involuntary and synchronous actions happen less than $1 \mathrm{~s}$ just before transporting the saliva into larynx. In contrast to radial encoding, which gave relatively robust images, conventional Cartesian encoding with comparable imaging parameters suffered from ghosting and blurring which obscure normal structures (arrows). Such motion-freezing snapshots from radial scheme could serve as a localizer technique before the real examination for all MRI applications, as shown later in Fig 5.4.
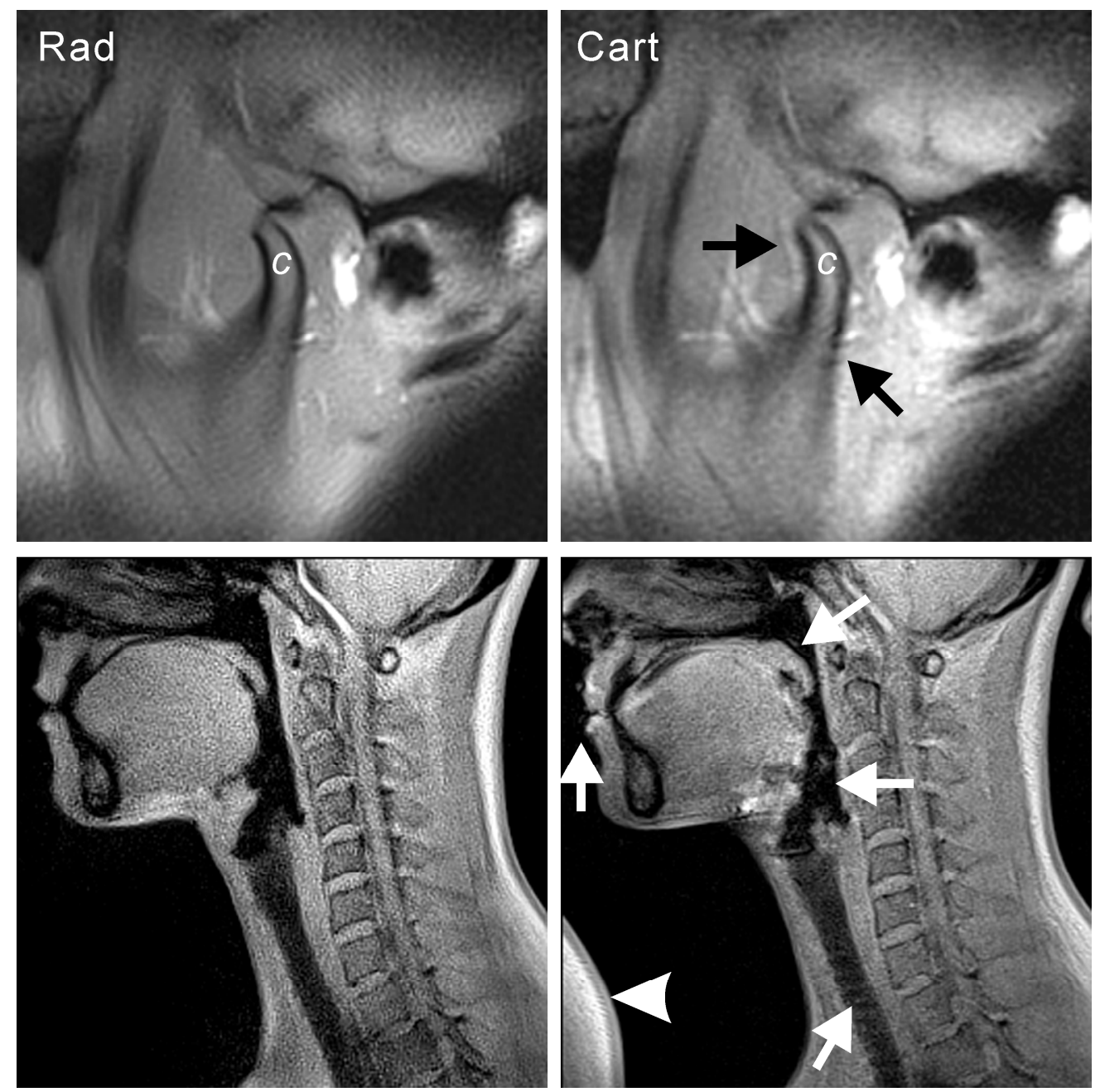

Figure 4.5 Motion robustness of radial encoding (left) in comparison to Cartesian encoding (right) during temporomandibular joint (TMJ) displacement (top) and swallowing of saliva (bottom). Note the motion ghosting artifacts (arrows) around the mandibular condyle (indicated by $c$ ) and in the oropharyngeal area, as well as the signal aliasing (arrow head) in Cartesian scheme. The imaging parameters: spoiled radial FLASH, FOV $192 \times 192 \mathrm{~mm}^{2}$, matrix size $256 \times 256$, in-plane resolution $0.75 \times 0.75 \mathrm{~mm}^{2}$, SLT $5 \mathrm{~mm}$ for TMJ in an oblique sagittal plane and $10 \mathrm{~mm}$ for swallowing in a middle sagittal plane, TR/TE 5.4/2.4 ms, FA 10 $B W 810 \mathrm{~Hz} /$ Pixel. For radial encoding, the view number was 265 with 5 interleaves.

As almost all difficulties and limitations vanish when replacing Cartesian with radial encoding, some disadvantages of a radial $k$-space scheme remain: First, it suffers from a 
pronounced sensitivity to the susceptibility induced off-resonance effects, which arises from its inherent trajectory formation with variable readout directions and $k$-space center intersections that result in demodulated phase errors and signal inaccuracies. Second, it is more sensitive to the deviations of the gradient time courses, which are caused by hardware imperfection such as improper timing alignment and eddy currents, and which result in additional phase errors. Such errors are also experienced in the conventional Cartesian scheme but can be safely neglected because all parallel encoded lines have the same error and will not affect the image quality, as illustrated in Fig 4.6a.
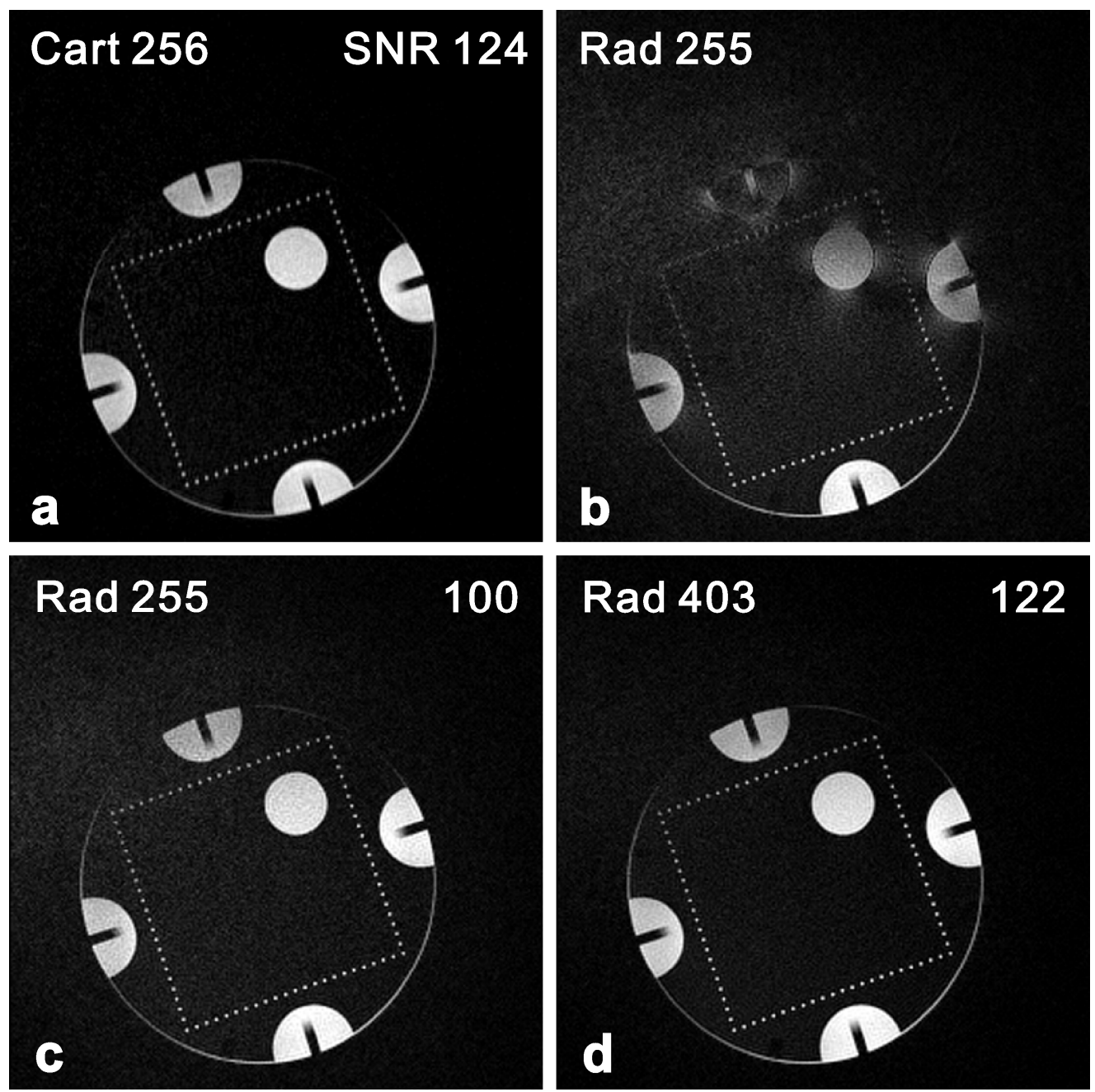

Figure 4.6 Comparison of SNR and off-resonance sensitivity between Cartesian and radial encoding for a phantom study. (a) Cartesian image without correction for off-resonance effects. (b) Radial image without correction for off-resonance effects. (c) Radial image with trajectory corrections for off-resonance effects. (d) Radial image with trajectory correction for off-resonance effects and with full sampling. Images were taken from off-center position with double oblique orientation. Concerning the SNR from (a) and (c), it shows that radial encoding yielded relatively lower SNR (radial/Cartesian: 100/124 $\approx 0.81$ ) with comparable encoding views (255 for radial / 256 for Cartesian). But when comparing (a) and (d), it shows that comparable SNR $(122 / 124 \approx 0.98)$ was obtained by radial encoding with full sampling (i.e. 403 views for 256 image matrix size). Off-resonance artifacts are hardly visible in the Cartesian image, but phase errors induced by the off-center position and eddy currents cause smearing as well as signal loss in the radial image without corrections, while they are effectively removed after corrections. 
It should be noticed that, although they cause smearing and signal loss in the radial image and thus may severely degrade the image quality, as shown in Fig 4.6b, both of these sources can be effectively compensated by applying proper corrections during the acquisition and encoding process, as demonstrated in Fig 4.6c. Details about the strategies and implementation of such corrections are given in Section 4.3.1 and 4.3.2. Third, as nonuniform grids, a certain decrease in SNR by a factor $\eta$ is applied based on the Eq 3.3. By counting in the sampled area, total number of samples and the weighting function (i.e. the inverse local density function, described in the next chapter) to measure the homogeneity of the sample distribution, this factor yields $\eta \approx 0.87$ [112]. Consequently, when using the same number of views or data points and the same pulse sequence (i.e. FLASH), the SNR of an image acquired with radial trajectories will be lower by about $13 \%$ compared to that with Cartesian where $\eta=1$, as shown in Fig 4.6. Further, radially sampled $k$-space needs a more complicated reconstruction algorithm, e.g. to perform data gridding before applying FFT. This will be introduced in the next Chapter.

\subsection{Specific Optimization for Time-resolved MRI}

Since the hardware (i.e. gradient system) and software (i.e. reconstruction algorithm) of most current clinical MRI systems are primarily optimized for Cartesian imaging, radial encoding may arouse image artifacts due to incomplete control of the measuring process. Therefore, in order to exert all efficiency of radial encoding with improved image quality for the development of RT-MRI, specific optimizations have to be implemented, as described in the following sections.

\subsubsection{View Number, Rotational Angle and Gradient Polarity}

First of all, the experimental accuracy is under the influence of how the entire views are organized in $k$-space, namely the number of views (either odd or even), angulation - maximal rotational angle that spread views in $k$-space (either $0^{\circ}$ to $180^{\circ}$ or $0^{\circ}$ to $360^{\circ}$ ), and the readout direction of the trajectories, which corresponds to the gradient polarity (either monopolar or bipoloar). Fig 4.7 presents a systematic comparison of various combinations of these three parameters. All images were taken at the iso-center position using a 32-channel head coil 
(Siemens AG, Erlangen, Germany) with applied trajectory corrections (see Section 4.3.2).

Firstly, even view number covering $360^{\circ}$ resulted in two-fold data redundancy and angular undersampling $\left(\Delta \emptyset=2 \pi / N_{V}\right)$ in the $k$-space distribution, which led to streaking artifacts in the image (black arrows) independent of the gradient polarity (Fig 4.7c and d).

Secondly, when comparing even or odd view number on a $180^{\circ}$ distribution with monopolar gradient to that with bipolar gradient (i.e. comparing a to b, e to $\mathrm{f}$ in Fig 4.7), $k$ space distribution of $180^{\circ}$ without gradient oscillation (i.e. mono-polarity, as in a and e) showed smeared images while the same distribution scheme with gradient oscillation (i.e. bipolarity, as in b and f) gave a clean result, independent of the view number. This can be explained by the theory of the off-resonance sensitivity from the radial encoding. In the conventional Cartesian case, any off-resonance phase error [172] is identical for all acquired lines because the signal is recorded during a constant velocity movement in the readout direction. Therefore, the resulting overall linear phase modulation, and consequently a translation (i.e. shift) of the affected areas in the image, will be present along this direction [173]. But since the readout time is short, the distortion of the object is tiny and usually hardly noticeable [128], as demonstrated in Fig 4.6. However, in the case of radial encoding, as the sampled views rotate, the experienced linear phase modulations cause a shift of the encoded spatial information with different orientations in the $k$-space and result in smearing or blurring in the image (white arrows). In this case, the corresponding point-spread-function (PSF) does not show itself as an ideal central peak any more but depends on the coverage scheme how the trajectories are organized. In the case of $\pi$ maximal rotational angle (i.e. $180^{\circ}$ ) without oscillating the readout direction, the shifts are one-sided which causes smearing of the reconstructed object (Fig 4.7 a and e); in the case with readout direction oscillation, the full $k$ space coverage yields a symmetrical distribution of the phase errors, so that the smearing is less visible in the image (Fig $4.7 \mathrm{~b}$ and f). Noticeably, this desired condition can also be achieved with a maximal rotational angle of $2 \pi$ (Fig $4.7 \mathrm{~g}$ and $\mathrm{h}$ ).

Thirdly, when comparing $180^{\circ}$ distribution with odd number views and with gradient oscillation (Fig 4.7f), 360 distribution with odd number views and without (Fig 4.7g) or with (Fig 4.7h) gradient oscillation, all three of them yield symmetric distribution of phase errors over the entire $k$-space. But they differ in the temporal ordering, i.e. incremental angle $\Delta \emptyset_{I N C}$, of the individually sampled views. For the purpose of a homogeneously incremented angle (to be explained later), the $180^{\circ}$ coverage with odd number views and with gradient oscillation (Fig 4.7f) was discarded. For the purpose of an efficient increment, the $360^{\circ}$ coverage with 


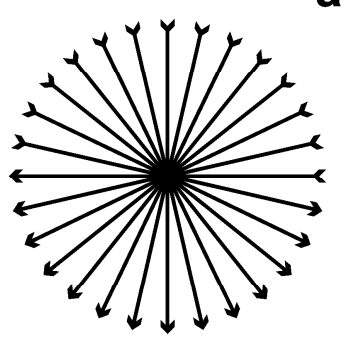

$\pi / N_{V}$

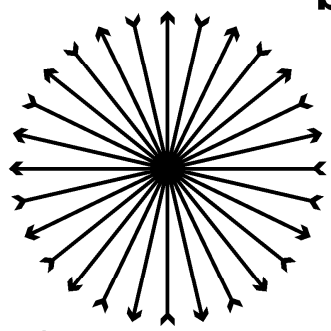

b
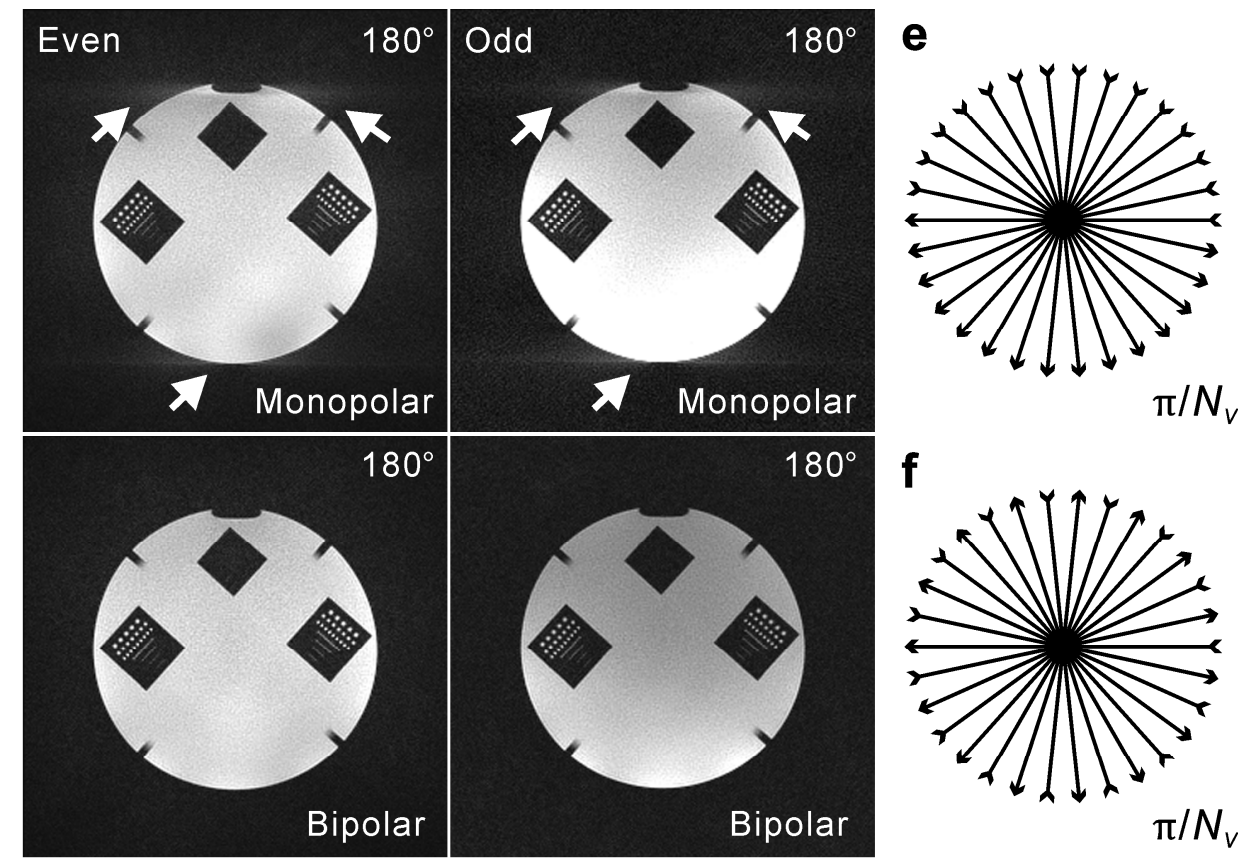

f

$\pi / N_{V}$

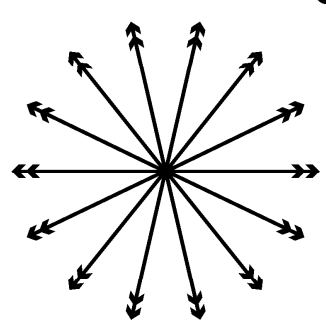

C

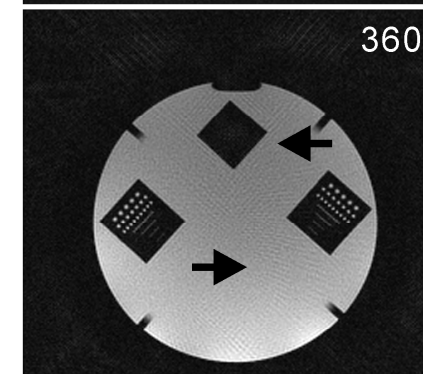

$360^{\circ}$

$2 \pi / N_{V}$

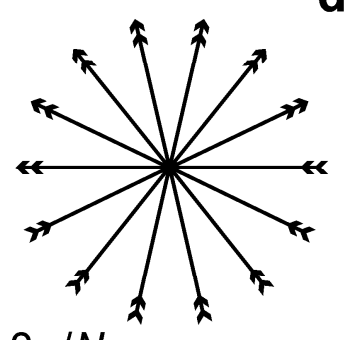

d
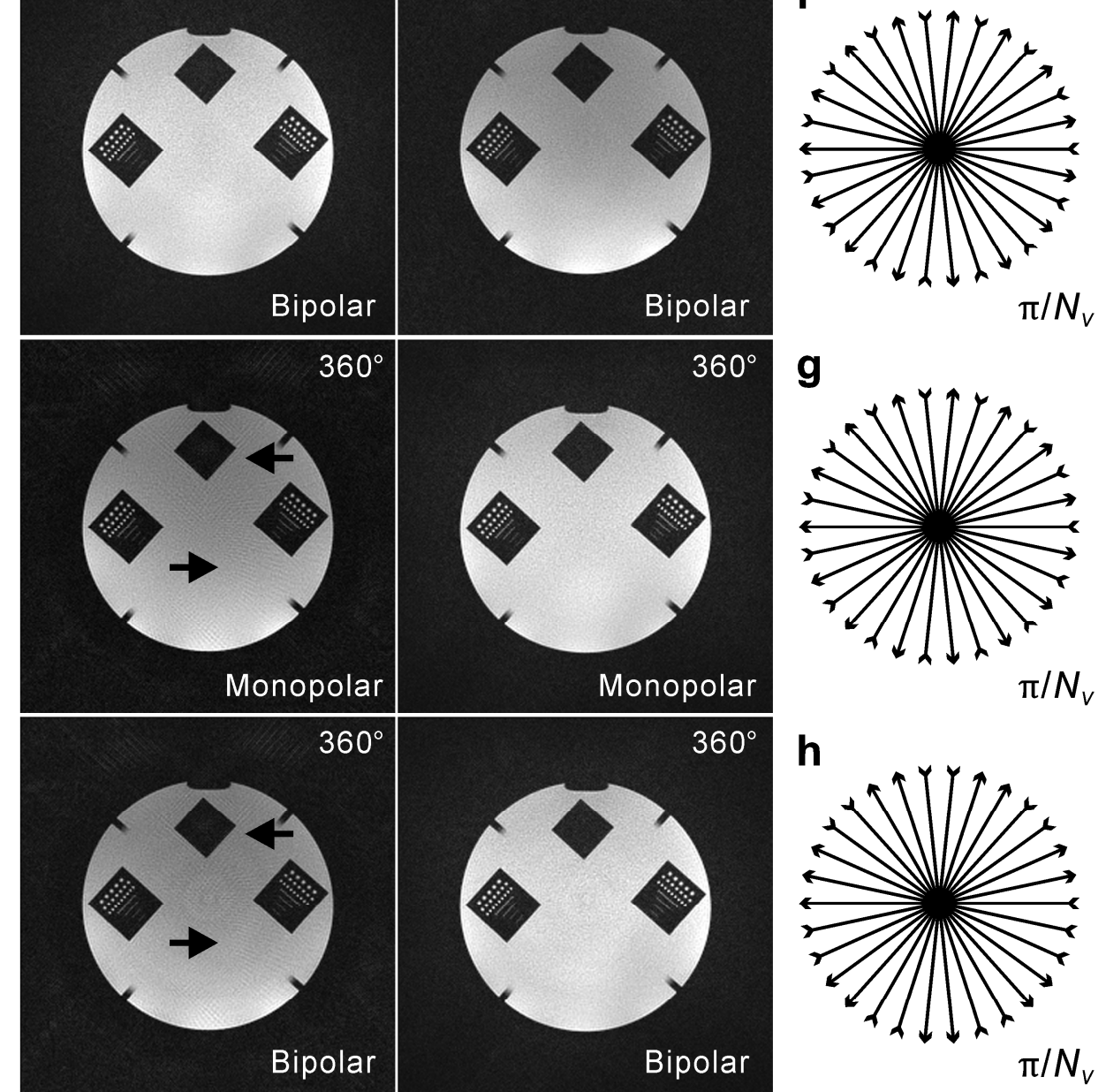

g

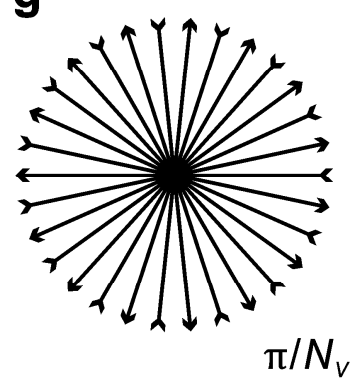

$360^{\circ}$

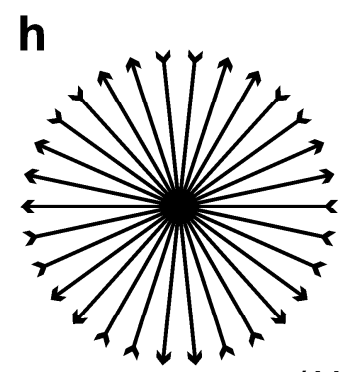

$\pi / N_{v}$

Figure 4.7 Influence of the number, rotational angle and readout direction of views in radial imaging for a phantom study. (a) to (d) were acquired with even view number $\left(N_{V}=204\right)$ for which 14 views were illustrated for simplicity in the corresponding $k$-space trajectories. (e) to (h) were acquired with odd view number $\left(N_{V}=205\right)$ for which 15 views were illustrated. For both cases, two schemes for $k$-space coverage (i.e. $180^{\circ}$ and $360^{\circ}$ ) and two readout directions (gradient polarities) were studied. Arrows in the trajectories indicate the readout direction and angle distance between each view $(\Delta \emptyset)$. White arrows indicate susceptibility artifacts induced by subject-related phase errors; black arrows indicate the streaking artifacts caused by angular undersampling.

odd number views and gradient oscillation where $\Delta \emptyset_{I N C}>180^{\circ}$ (Fig 4.7h) was also discarded. Therefore, an encoding scheme of either $180^{\circ}$ coverage with even number views combining gradient oscillation or $360^{\circ}$ coverage with odd number views without gradient oscillation is 
the optimal choice to achieve good image quality, at least in static imaging. As a matter of fact, with respect to the trajectory, an optimal combination would be that each view possesses an opposed encoding direction to its neighboring view.

\subsubsection{Trajectory Correction}

Apart from the object-related off-resonance sensitivity, additional phase modulations caused by hardware imperfections need to be separately corrected, because they do not depend on the gradient polarity and thus cannot be compensated by alternating the gradient readout direction. These system-induced phase errors mainly arise from two sources: gradient delays due to eddy currents, and an improper timing alignment between frequency demodulation reference signal and the real data acquisition. Such problems are also experienced in Cartesian imaging [174], but can be safely ignored as explained before (Fig 4.6). In a radial encoding scheme, they both cause trajectory deviations in $k$-space, and correspondingly, object blurring and signal cancelation in the reconstructed image.

First of all, gradient delays may introduce time discrepancies, for example, between playing out gradient moments and real signal acquisition. They will cause a deviated time course of the actually experienced field strength [128] and produce substantial phase errors in the acquired signal [175]. These lead to displacement of views parallel to their readout directions as well as in the perpendicular direction, so that they do not even pass the $k$-space center exactly [135].

Secondly, a reference signal $S_{\text {ref }}$ is required for imaging off-center slices when using timevarying gradient amplitudes, as in radial imaging. It is created by the system and used to multiply the spin signal to get the actual off-center image:

$$
S_{r e f}=e^{-i(\Delta f \cdot t+\varphi)}
$$

where $\Delta f$ is the modulated frequency, which changes according to the applied gradient waveform $G$ and the shift $d_{x}$ of the FOV from the gradient iso-center:

$$
\Delta f=\gamma \cdot G \cdot d_{x}
$$

assuming $d_{x}$ is the offset along $\mathrm{x}$-axis in the readout direction, and $\varphi$ is the initial phase which is calculated by the system such that the phase of the reference signal equals zero at time $t_{D C}$ when the acquired data point $D_{D C}$ corresponds, i.e. by FFT, to the $k$-space center [176]. Any inaccurate synchronization between acquisition and reference signal makes this phase dependent on $\Delta f$ and thus on the offset $d_{x}$. Therefore, for imaging off-center slices in radial 
encoding when $d_{x} \neq 0$, this results in multiplications of each view with a different reference signal, and such trajectory deviation and phase variation further results in artifacts in the image.

To correct for these errors, different approaches have been suggested, either by gridding reconstruction [177, 178] or by adjusting each view from additional measurements for characterization of echo shift $[179,180]$, based on the calibration of the trajectories to assess the gradient deviations [175]. Fortunately, although such delays are normally anisotropic and are dependent upon different gradient axes [175] as can be seen from the above equations, it has been shown that for specific MRI system, deviations mainly occur as isotropic delays that only depend upon $t_{o s}$, which stands for the receiver dwell time including oversampling in $\mu \mathrm{s}$ $[176,181]$. Thus, they can be well approximated by a linear equation. For the system described in Section 2.4, a time shift was added to adjust the readout prephase (or dephase) gradient, and also the rephase gradient for refocused radial FLASH sequence, to compensate trajectory errors in the first situation [181]:

$$
\Delta t_{1}=0.42 \cdot t_{o s}-3.90 \mu s
$$

while another time shift was incorporated into the phase of reference signal to compensate trajectory deviations in the second situation [176]:

$$
\Delta t_{2}=0.5 \cdot t_{o s}+2.0 \mu \mathrm{s}
$$

In Fig 4.8, the acquired phantom images without corrections clearly demonstrate how these small time delays degrade radial image quality with significant artifacts. For both iso-center and off-center positions, the above carefully selected radial encoding schemes were applied. However, images acquired at iso-center position with signal void and also streaking (black arrows) showed that such system-related timing errors could not be compensated by simply using opposed encoding directions of neighboring views. Images acquired at off-center position showed additional smearing (white arrows) due to improper timing alignment of reference signal and receive event. To the right side, the corresponding corrected images show that all artifacts were successfully removed for both radial encoding schemes. This could facilitate radial imaging in applications with variable slice positioning, e.g. in cardiac or interventional imaging when off-center double-oblique slice orientations are often encountered. Because this procedure is sufficient to obtain high-quality images with radial sampling, the concept was employed in all experiments of this thesis. 
w/o Traj. Corr.

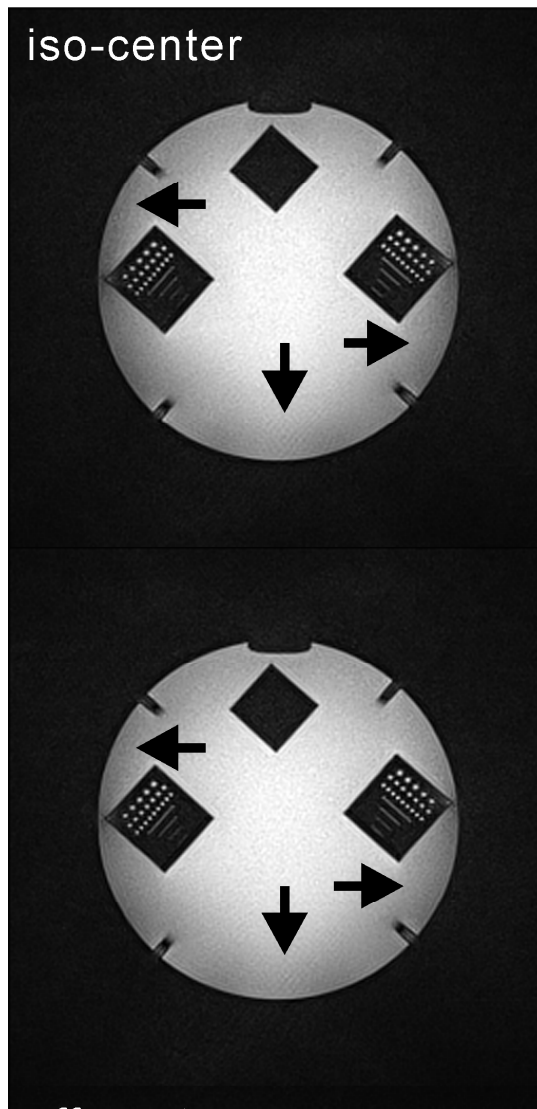

off-center

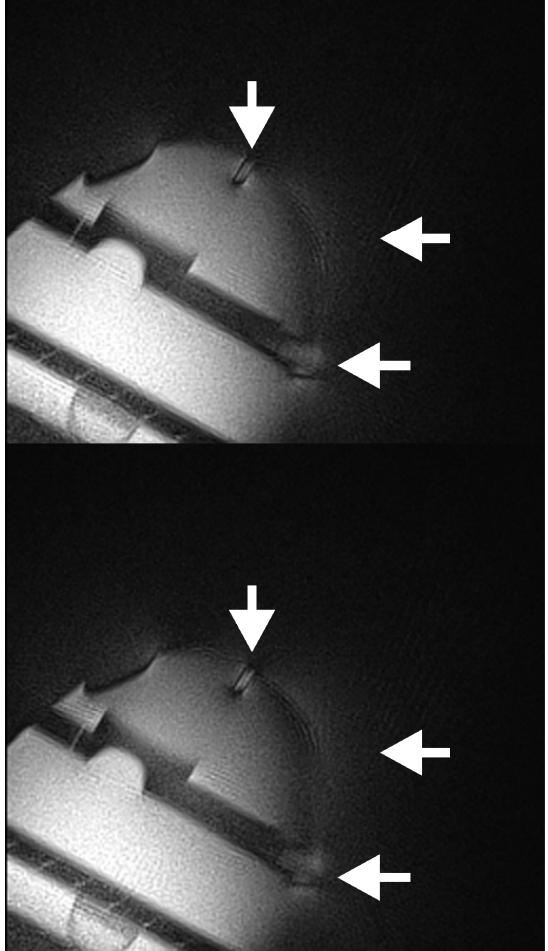

with Traj. Corr.
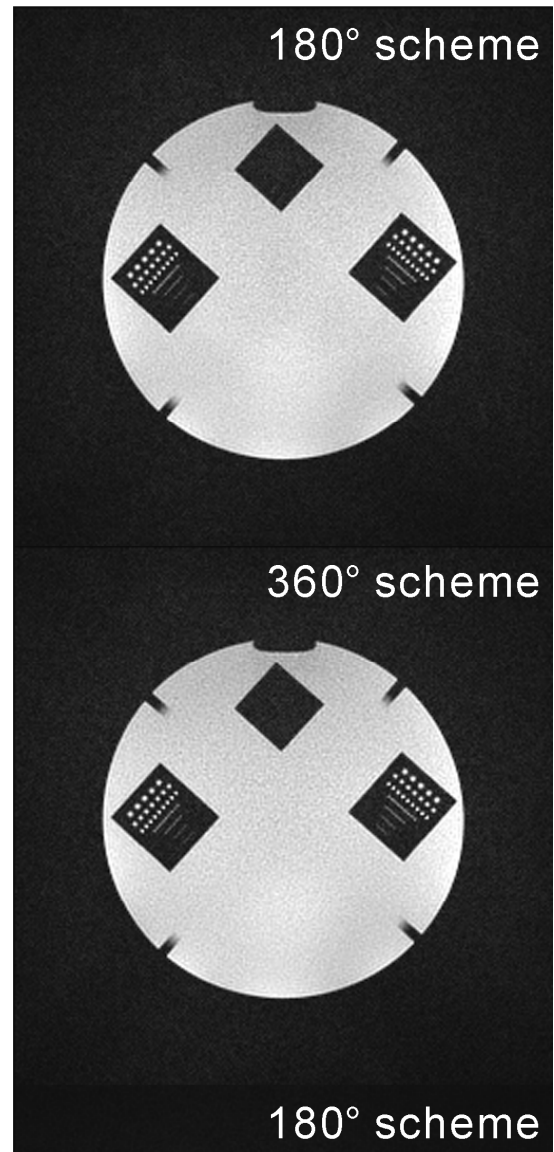

$180^{\circ}$ scheme
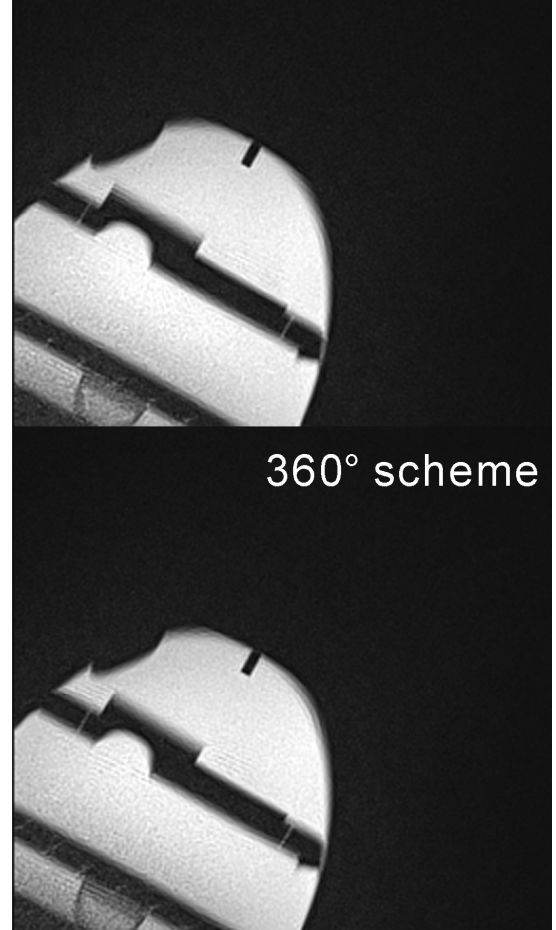

Figure 4.8 Trajectory corrections for radial encoding for a phantom study. Two conditions were investigated - iso-center position in a transversal plane and off-center position in a double oblique plane. For each condition, two optimal sampling patterns, i.e. $360^{\circ}$ coverage with odd number of views and $180^{\circ}$ coverage with alternated even number views, were applied. Black arrows and white arrows indicate signal void and additional smearing, respectively, both induced by system-related phase errors. 


\subsubsection{View Reordering}

The impact of the view order on achieving time-resolved imaging, in dynamic studies of timevarying processes, is discussed here. The view order indicates the order, in which sequentially acquired views are arranged in $k$-space. It is known that motion would be smooth if the induced signal inconsistencies could be kept as low as possible between adjacent acquired views. However, this is often not the case during dynamic studies with, for example, impulsive blood flow or rapid myocardial movements. Therefore, it is recommended to "geographically" divide, i.e. to interleave, the individually acquired views, with a uniform distribution that cover the entire $k$-space during the encoding of one image to minimize motion artifacts.

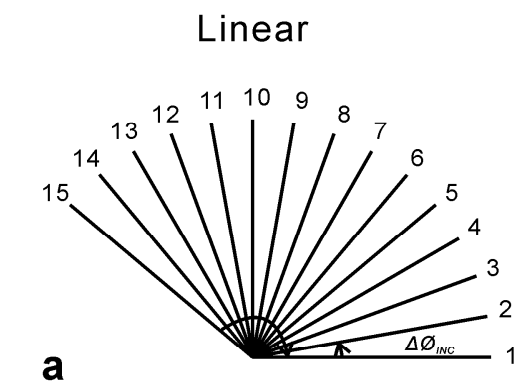

\section{Sequential Interleaving}

\section{Reordered Interleaving}
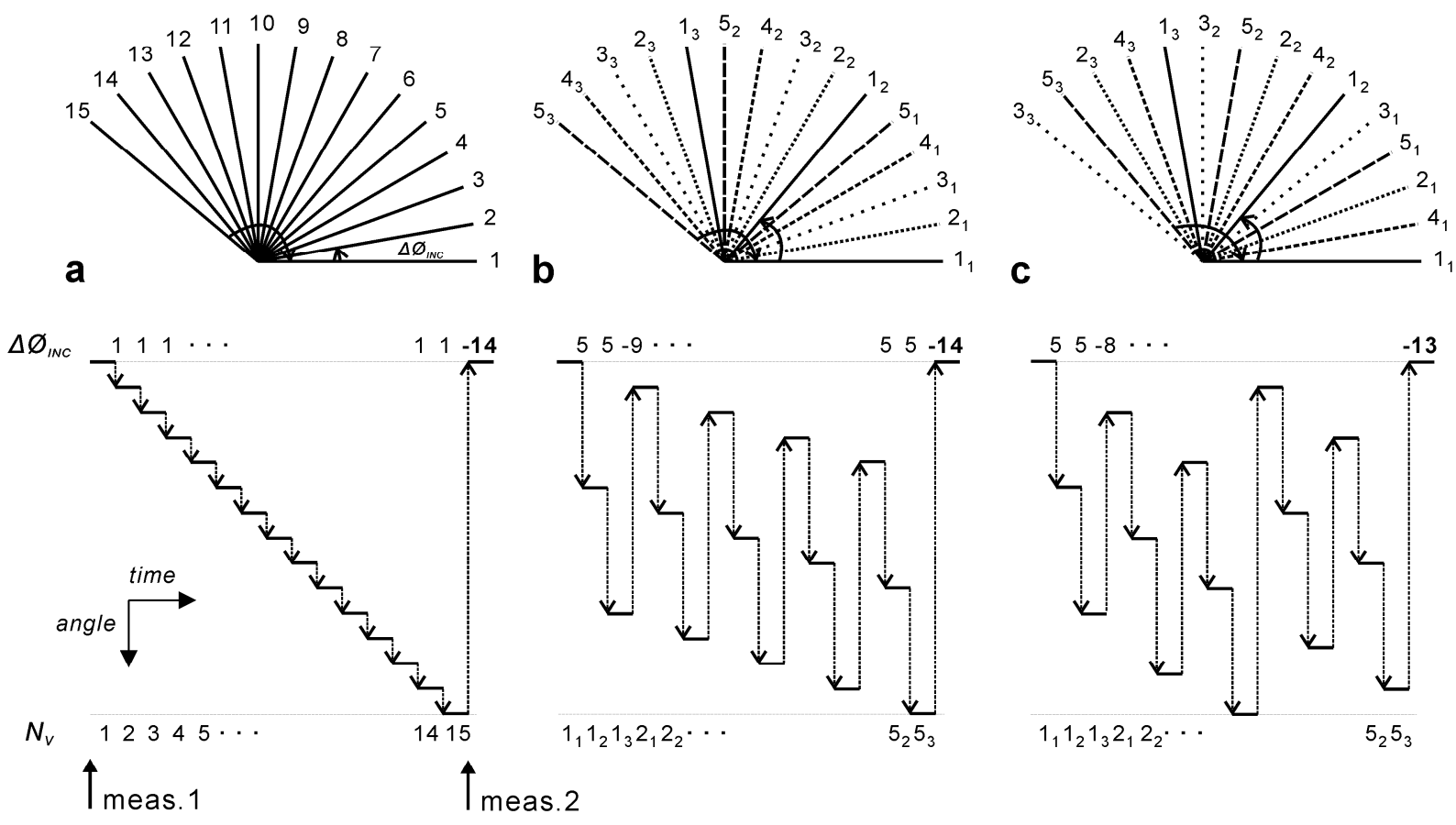

d

e

f

Figure 4.9 Schematic diagram of different view reordering in radial $k$-space encoding. For simplicity, 15 views for a $360^{\circ}$ scheme were illustrated with $\Delta \emptyset=10^{\circ}$ distribution. In a (a and d) normal linear order radial encoding, the angle increment $\Delta \emptyset_{I N C}$ equals the azimuthal view gap $\Delta \emptyset$, but has a large jump of $\left(N_{V}-1\right) \cdot \Delta \emptyset$ from one image frame to the next. In a (b and e) sequential interleaving (with five interleaves), $\Delta \emptyset_{I N C}$ within one image frame is more uniformly distributed over the $k$-space, but still has the same jump as in the linear case. In the (c and f) reordered interleaving (with five interleaves), each view is arranged with optimal distribution not only within the acquisition of one image frame, i.e. encoding of one corresponding $k$-space, but also over the entire repeated measurement.

Several proposals have already been made to optimize the view order, in order to improve temporal fidelity of radial encoding. The most common approach is to simply acquire views 


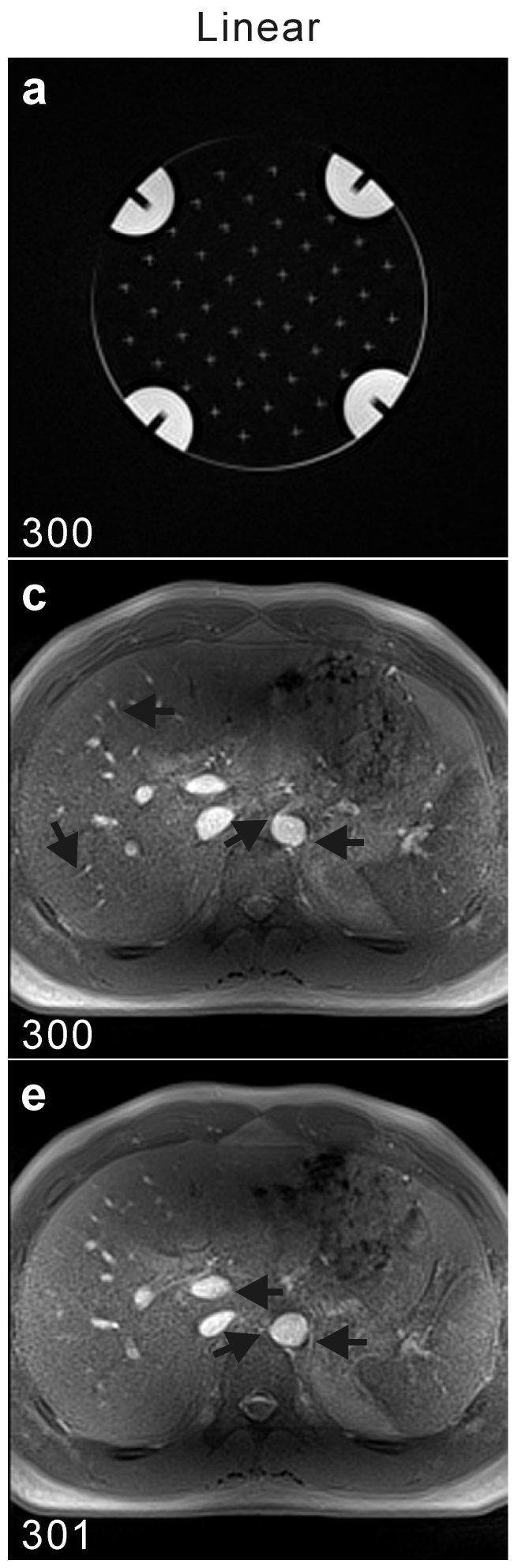

\section{Reordered Interleaving}
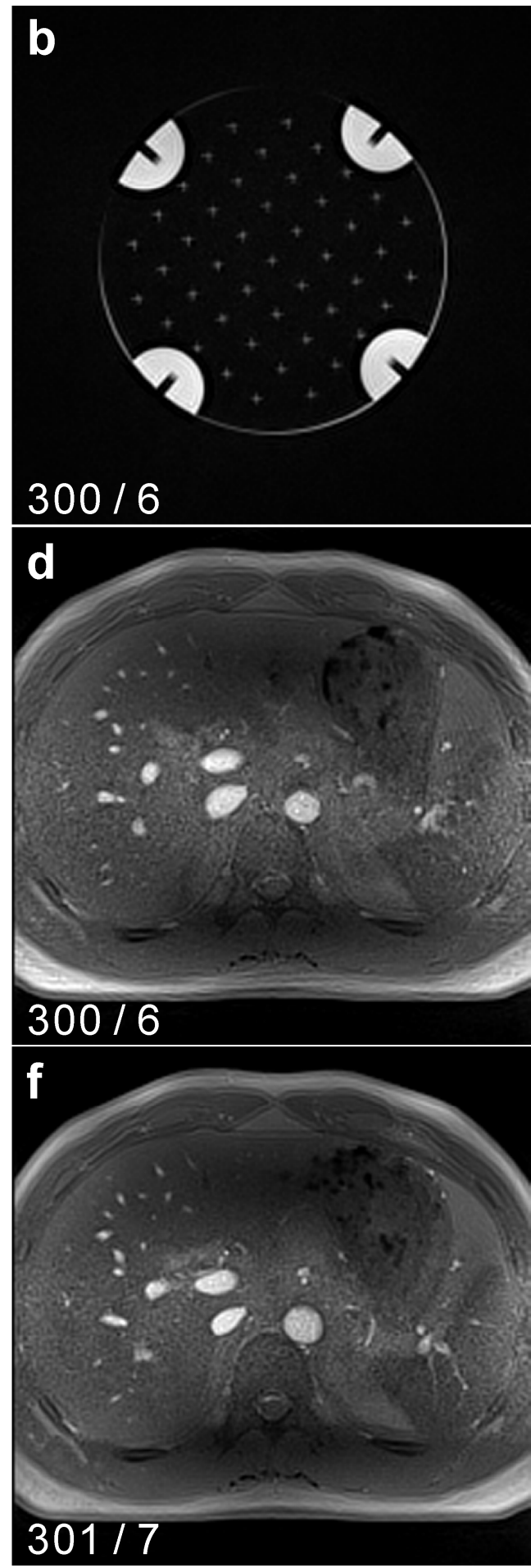

Figure 4.10 Experimental comparison between linear (left) and interleaved radial encoding (right, described as reordered interleaving in Fig 3.9), for a (a and b) phantom and a (c to f) human abdomen (healthy volunteer, male, 28 years old) in a transversal plane across the liver during free breathing. Images in (a, b, $\mathbf{c}$ and $\mathbf{d})$ were acquired by $180^{\circ}$ scheme; images in (e to f) were acquired by $360^{\circ}$ scheme. Black arrows indicate motion induced artifacts. Imaging parameters used here were identical for both approaches: refocused radial FLASH, 32-channel body array coil (Siemens AG, Erlangen, Germany), FOV 256×256 $\mathrm{mm}^{2}$, matrix size $192 \times 192$, in-plane resolution $1.3 \times 1.3 \mathrm{~mm}^{2}$, SLT $8, T R / T E 5.0 / 2.5 \mathrm{~ms}$, FA $12^{\circ}, B W 640$ Hz/Pixel. 
in a segmented scheme with certain interval in azimuthal direction (Fig 4.9b), here termed as sequential interleaving to distinguish it from other interleaving patterns [182-187].

Compared to the conventional linear order, either linear ascending $\left(360^{\circ}\right.$ sampling scheme with odd number views) or linear alternate scheme $\left(180^{\circ}\right.$ sampling scheme with even number views), the motion discontinuities are reduced between $0^{\circ}$ and $360^{\circ}$ within acquisition of one image. However, it still remains a large jump in incremental angle $\Delta \emptyset_{I N C}$ from acquisition of one image to the next. Indeed, since the acquisition order of the first view in each segment (e.g. from $1_{1}$ to $5_{1}$ in Fig 4.9b) is a linear order, this large jump is always experienced from the last view in the last segment of one image ( $k$-space) to the first view in the first segment of the next (Fig 4.9e). Because consecutively encoding the $k$-space with multiple azimuthal segments is equivalent to repetitively encoding with these multiple turns (i.e. segments) in series, all turns not only within the acquisition of one image but also between images are desired to be distributed uniformly. Therefore, an advanced interleaving scheme, called reordered interleaving here, was developed in this thesis. Note from Fig 4.9c and $\mathrm{f}$, the acquisition order of the first view in each segment is also interleaved. In such a way, sequentially acquired segments in time result in a more uniform view distribution over the entire dynamic imaging process, so that motion induced inter-view fluctuations are reduced to minimal. It is similar to previously reversed bit order [188] but is not restricted by the choice of the number of the segments, and applies for both $360^{\circ}$ and $180^{\circ}$ schemes.

Several experimental comparisons of linear and reordered interleaving approaches in radial encoding are presented in Fig 4.10 and Fig 4.11. The view number $N_{V}$ and the interleaves (i.e. segment) number $N_{\text {seg }}$ are given directly in the figures. First of all, images in Fig $4.10 \mathrm{a}$ and $\mathrm{b}$ show that, for static imaging, both approaches yield same result and quality. Second, images in $4.10 \mathrm{c}$ and $\mathrm{d}$ were acquired by the $180^{\circ}$ scheme, while images from e to $\mathrm{f}$ were by the $360^{\circ}$ scheme, according to the conclusion in Section 4.3.1. All were applied with trajectory corrections according to the descriptions in Section 4.3.2. They show that both $180^{\circ}$ and $360^{\circ}$ schemes benefit from the reordering strategy in resolving small changes such as the structures and edges of the veins (black arrows) in the transversal liver imaging during pulsile motion and free breathing. In addition, numerous dynamic imaging studies have shown that image quality from the $360^{\circ}$ scheme is slightly better than that from the $180^{\circ}$ scheme, although it is not well recognized here by comparing $4.10 \mathrm{~d}$ and $\mathrm{f}$. Third, images from $4.11 \mathrm{a}$ to $\mathrm{d}$ were acquired by the $360^{\circ}$ scheme. They show that, by employing view reordering optimization, the robustness of the moving structures with large contrast changes such as cardiac motions (white arrows), is also dramatically improved. 


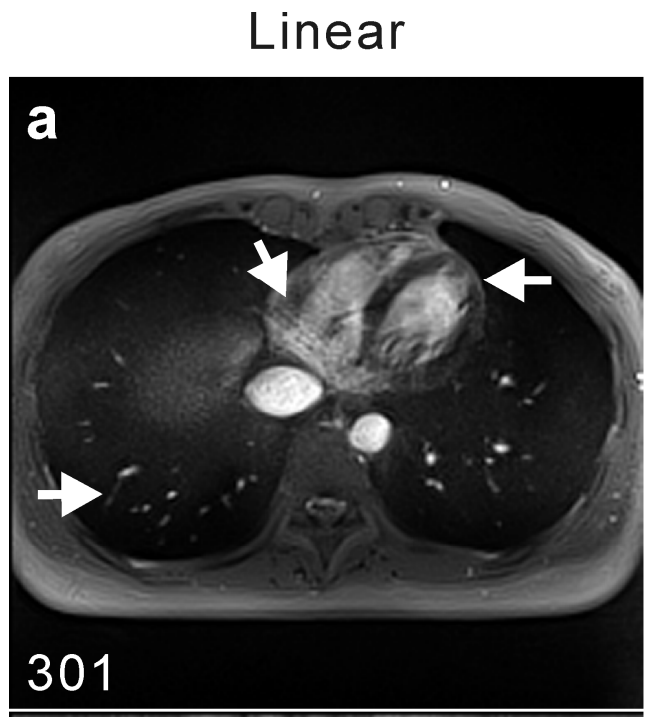

\section{Reordered Interleaving}
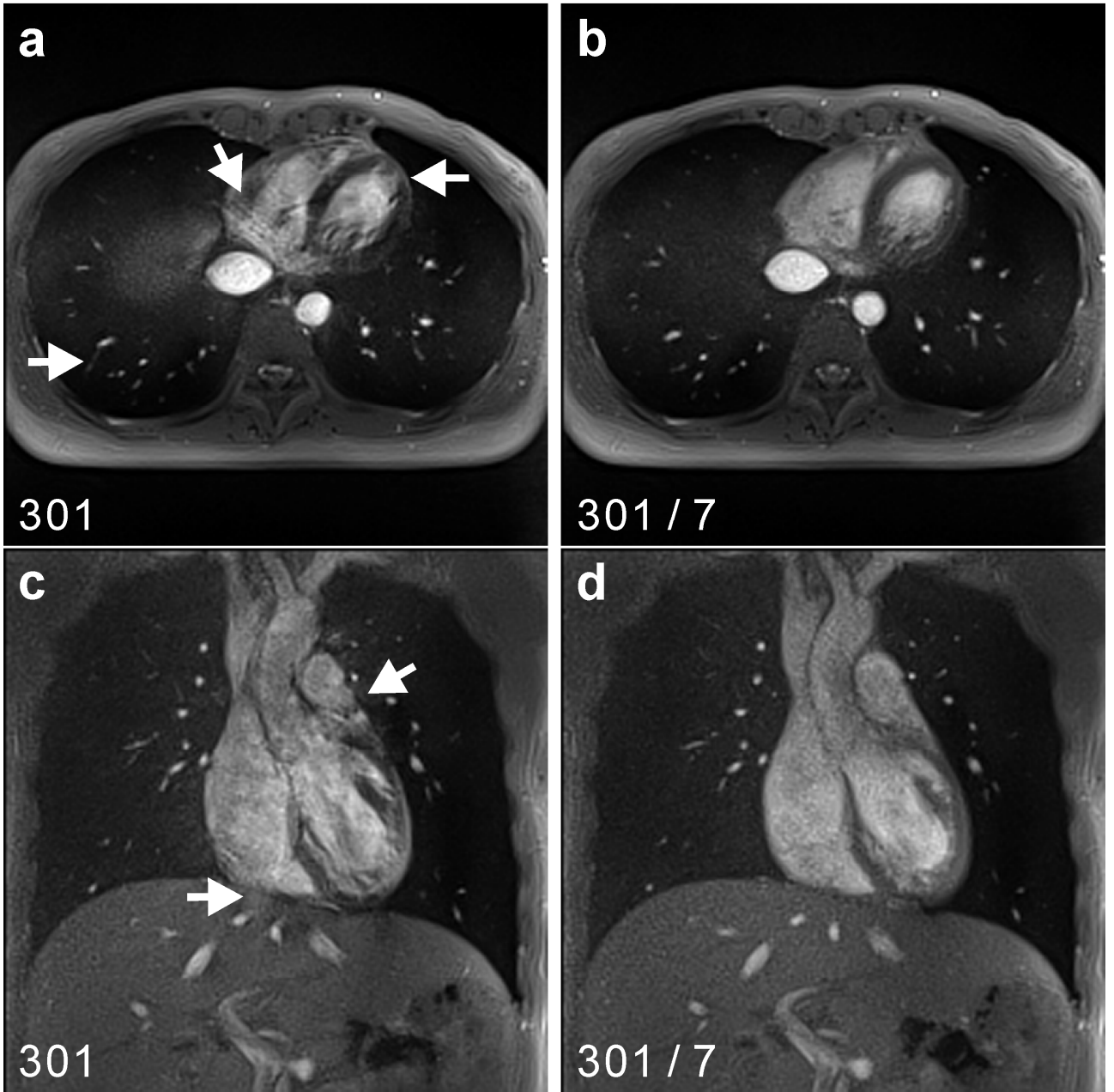

Figure 4.11 Experimental comparison between linear (left) and interleaved radial encoding (right, described as reordered interleaving in Fig 3.9) for a human cardiac imaging study (healthy volunteer, female, 28 years old) in a (a and b) transversal and (c and d) coronal plane during free breathing and without ECG synchronization. Images were acquired with a $360^{\circ}$ scheme. White arrows indicate motion induced artifacts. Imaging parameters were the same as that in Fig 4.10, except for the use of spoiled radial FLASH.

\subsection{Summary}

Based on the FLASH technique, different $k$-space encoding schemes can be used. The traditional Cartesian encoding with rectilinear scheme has the typical disadvantages of 1) sensitivity to undersampling with signal aliasing and blurring; 2) sensitivity to motion with ghosting and smearing; 3) unequal encoded lines with either low or high spatial frequencies. This becomes a substantial problem for MRI of dynamic processes and with presence of unavoidable motions from patients, which cause errors in the recorded signal and finally translate as artifacts in the images that may obscure the object. On the other hand, radial 
encodings benefit from unique properties: 1) undersampling insensitivity with mild blurring and streaks at worst case; 2) free zooming due to oversampling in all radial directions; 3) motion robustness due to absence of phase encoding and intrinsic oversampling in the $k$-space center; 4) each acquired view with equivalent information containing both low and high spatial frequencies which is best suited for sliding window and view sharing techniques. Therefore, it is concluded that the radial scheme is used for efficient spatial encoding and for imaging time-varying processes. It has been shown that the radial encoding is superior to other trajectories based on the same imaging condition, and this has led to applications for motion artifact reduction and fast data acquisition in contrast-enhanced imaging $[28,29,182$, 183, 189-192].

For the development of RT-MRI, several specific optimizations are done to compensate for the drawbacks of radial encoding, based on which the image characteristics were analyzed and appraised: 1) By applying specific sampling pattern of $360^{\circ} k$-space coverage with odd number views, as well as trajectory corrections, both subject-related (i.e. susceptibility induced off-resonance effects) and system-related (i.e. hardware imperfection) phase errors, which cause unneglectable trajectory deviations in radial encoding, were successfully compensated. 2) By applying reordered interleaving strategy to uniformly distribute the acquired views in the $k$-space to minimize motion inconsistencies and smear out induced artifacts, considerable improvements in the radial encoded images were visible and timeresolved MRI was achieved.

In the next chapter, the gridding algorithm, which effectively solves the drawback of the complicated reconstruction, is described. More importantly, a comprehensive discussion of the sliding window and view sharing techniques in combination with interleaved radial encoding is given, to further improve the temporal resolution for the development of RT- MRI. 


\section{Chapter 5}

\section{Development of Real-time MRI: Image Reconstruction}

MR image reconstruction is the mathematical process that transforms the acquired $k$-space data into the desired image. Proper algorithms have to be chosen according to the signal acquisition and encoding schemes, in order to obtain images that are accurate and fast. Therefore, at the beginning of this chapter, the theoretical concepts of the gridding approach, which was used in this thesis for radial encoding, is briefly described.

Normally, the MRI reconstruction process includes certain delays, which mainly refer to signal acquisition if data transfer in the local network is neglectable. To further accelerate this process, the image reconstruction needs to be decoupled from the acquisition. Thus, as the main focus of this chapter, the principle of sliding window with view sharing technique is explained. It allows for an increase of the image reconstruction rate and a smooth update of the images in real time. Practical demonstration from experimental implementations is presented and analytical comparison with other image reconstruction strategies is also given.

\subsection{Reconstruction with Gridding}

To reconstruct images from the $k$-space data that are radially encoded, two aspects should be considered and properly handled: 1) the non-gridded sampling positions and their 2) varying densities. Two different approaches that are most commonly used are filtered back-projection (FBP) algorithm and convolution gridding reconstruction. The former is based on the projection-slice or central-slice theorem [6, 32, 33]. A projection is the 1D inverse Fourier transform of a sampled $k$-space line (radial view) with certain gradient direction (view angle at $\varnothing)$ and represents the line integrals along the direction perpendicular to that gradient direction (projection direction at $\varnothing+\pi / 2$ ), which yield the projection profile of the object. By applying inverse Radon transform to the continuous set of projections at all directions after filtering the profiles with an M- or ramp filter $|k| / N_{s}$ to avoid the typical blurring of a back- 
projection, an image can be obtained.

The FBP method was the only applicable technique for reconstructing radial MRI data before the gridding (also called regridding) approach was introduced in medical imaging in 1985 [30]. Basically, the gridding method involves firstly a convolution of each non-Cartesian sampling point with a dedicated kernel and re-sampling or interpolation at appropriate Cartesian grid locations, and secondly a normal inverse 2D-FFT for the reconstruction.

As the effects of these two methods can be regarded equivalent [128], the following section will focus on the latter, which was used in this thesis.

\section{Density Compensation}

In the first step, the varying sampling density of radial $k$-space data is compensated by the density weighting function or density compensation function (DCF) before interpolation to Cartesian grids. The intrinsic inhomogeneous distribution of the radially acquired samples in the $k$-space yields a sample density function $D(k)$, which describes how densely or sparsely the samples are distributed. It can be expressed by the inverse area underlying each sample $[27,193]:$

$$
D(k)=\left\{\begin{array}{cc}
2 N_{V} / \pi, & k=0 \\
N_{V} / \pi|k|, & k \neq 0
\end{array}\right.
$$

Therefore, the density correction is achieved by weighting each of the acquired samples with a density compensation function (DCF), which is defined as the inverse local density function, i.e. the inverse density of the sampling positions in the $k$-space [194]. For radial or spiral schemes as closed-form trajectories, such a DCF $W(k)$ can be analytically derived, which yields a Ram-Lak filter for radial [195, 196]:

$$
W(k)=D_{R L}(k)= \begin{cases}\pi /\left(2 N_{V}\right), & k=0 \\ \pi|k| / N_{V}, & k \neq 0\end{cases}
$$

DCFs for more exotic trajectories such as rosette or stochastic encoding must be determined numerically [197], for example, estimated by computing a Voronoi diagram of the trajectory $[193,198]$. In this case, the choice of the DCF may greatly influence the final image, and the convolution gridding is also non-trivial to perform. This is another reason why the simple radial encoding is preferable compared to those complicated ones, besides the technical implementation problem that has been mentioned in the last chapter.

\section{Convolution Gridding}

In the second step, all density corrected or compensated data $S_{D C}(k)=S(k) \cdot W(k)$ are re-sampled 
to equidistant Cartesian grids. This is achieved by convolving each sample point with a small interpolation kernel $K$ and then assigning the interpolated data, which lie within the kernel, to the desired grid points:

$$
S_{G R D}(k)=\sum_{i} S_{D C}\left(k_{i}\right) * K\left(\left|k-k_{i}\right|\right)
$$

where $\left|k-k_{i}\right|$ denotes the distance between the $i$ th sample and the desired grid point, $K\left(\left|k-k_{i}\right|\right)$ is the window function of the interpolation kernel, and $S_{G R D}(k)$ is the resultant signal, which is a weighted sum over all sample points within this kernel window.

Because this interpolation achieved by convolution in the frequency domain may lead to certain modulation effect in the image domain, the interpolation kernel has to be chosen adequately to make this effect tolerable. It is known that the multiplication of a compact signal with a rect function does not distort the signal (provided that the support of both functions is equal), an optimal interpolation kernel is then described in the frequency domain by a sinc function [30]. However, this convolution with such an infinite sinc function with unlimited support is computational unfeasible in practice. Hence, a Kaiser-Bessel window function [199] is proposed as the interpolation kernel to approximate the properties of the sinc function [200]:

$$
K(k)= \begin{cases}\frac{1}{\beta} I_{0}\left(\pi \alpha \sqrt{1-\left(\frac{2 k}{\beta}\right)^{2}}\right), & |k| \leq \frac{\beta}{2} \\ 0, & |k|>\frac{\beta}{2}\end{cases}
$$

where $I_{0}$ is the zero-order modified Bessel function of the first kind, and $\alpha$ and $\beta$ are constant free design parameters. $\alpha$ is an arbitrary real number that determines the window shape, and the integer $\beta$ is the window width. The $\beta$ value is usually between 3 and 5 to achieve good compromise between interpolation accuracy and time availability [200]. The larger the value of $|\alpha|$ is, the narrower the window is, whereas the wider the main lobe and the lower the amplitude of the side lobes in the FT of $K(k)$ become. Therefore, it should be well determined to control the tradeoff between main lobe width and side lobe area, for example, according to a previous study [201]. In all reconstruction operations in this work, a Kaiser-Bessel kernel width $\beta=3$ (in units of $\mathrm{m}^{-1}$ for a normalized FOV of $1 \mathrm{~m}$ ) and a shape parameter $\alpha=4.2054$ were chosen as suggested in [200].

\section{Inverse 2D-FFT}

In this step, a normal two-dimensional inverse FFT of the data grid is performed as in the Cartesian case as described in Section 2.2.3, and the details are not repeated here. 


\section{Roll-off Correction}

As addressed above, due to the convolution with a finite interpolation kernel, the obtained image exhibits an undesired modulation with the Fourier transform of the kernel. This is called roll-off effect. Therefore, a corresponding compensation method, also called deapodization, which involves dividing the intermediate image by the Fourier transform of the convolution kernel function, must be performed to obtain the final image.

\section{Image cropping}

Because the gridding process is done discretely, replications of the object occur in the image domain. To avoid signal aliasing between neighboring object copies (similar to the effect when employing discrete FT reconstruction) which may be amplified by the roll-off correction [128], a two-fold oversampling of the gridding matrix is often used. Therefore, the extended FOV is cropped at the end of the reconstruction procedure, as shown in Fig 5.1.
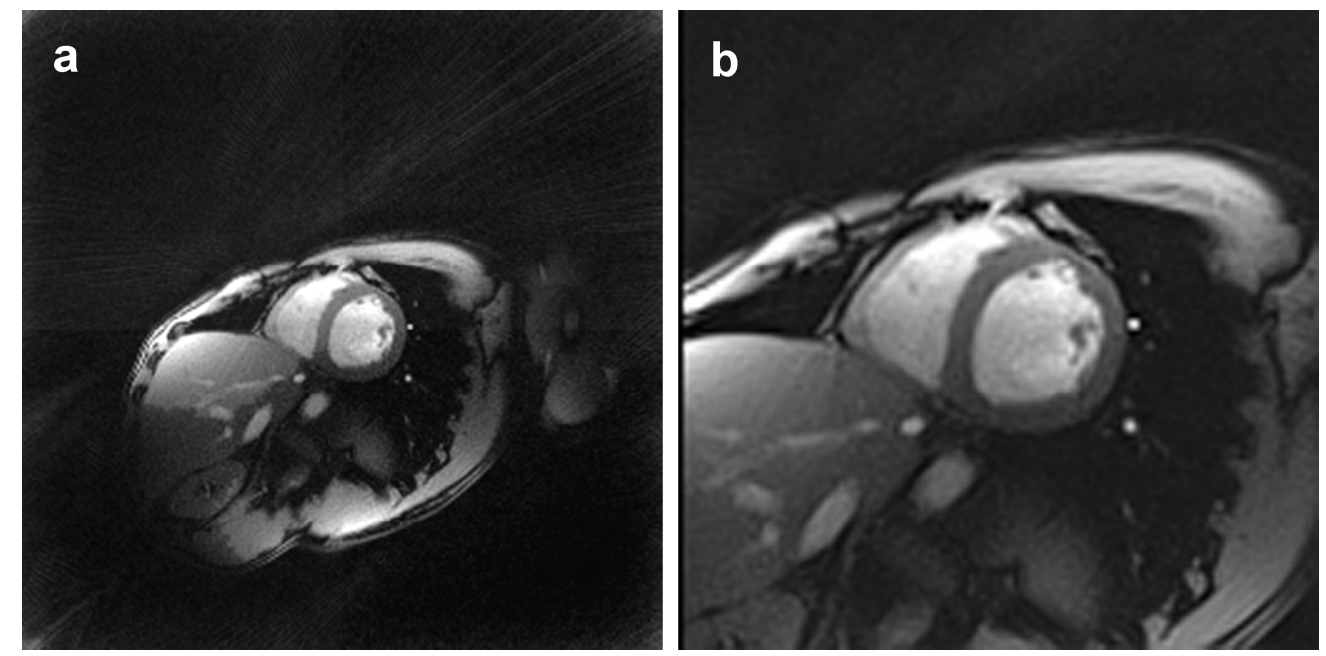

Figure 5.1 Radial gridding with 2-fold oversampling. The image was acquired during a cardiac RT-MRI experiment with an anatomically defined short-axis view. (a) Image with double FOV before cropping. (b) Image with selected FOV after cropping at the end of reconstruction.

In generally, although the gridding process is computationally intensive, with reasonable kernel window parameter values, as described above, the computation is fast enough to be combined with inverse FFT to reach real-time image reconstruction with good image quality, as demonstrated in Fig 4.3 and Fig 6.7, in static and dynamic imaging, respectively. 


\subsection{Update with Sliding Window and View Sharing}

The standard reconstruction approach performs the last step - transformation from raw data to image data - only when the whole $k$-space is refilled by newly acquired data. In other words, whenever the reconstruction buffer, corresponding to the chosen FOV or $k$-space, is filled by the acquired data, it is emptied by outputting the data to the image archive (e.g. for offline diagnostic usage) and is prepared for the next reconstruction cycle.

However, for real-time reconstructions in a fluoroscopic manner, which was employed in this thesis, it works in a different way. The reconstruction buffer contains sampled $k$-space data which, notably, is a mixture from the recent past (previous) acquisition and the latest (current) one. This is to say that whenever the reconstruction buffer is partially replaced (i.e. the oldest acquired data replaced by the newly acquired data), it outputs the data to the display screen (e.g. for online observation and monitoring) and prepares for the next reconstruction cycle (i.e. replacement of the newly acquired data with the old one), but is never emptied until the end of the entire measurement. Thus, the contents of the reconstruction buffer are updated, reconstructed, and displayed continuously at a higher rate, which is described as frame rate, as in video technique. It defines the number of images that are reconstructed and displayed per second and is regarded as the apparent or effective temporal resolution with a unit of frames per second (fps) or hertz $(\mathrm{Hz})$. It is equal to the reciprocal of the update time (TU) and should be distinguished from the true temporal resolution which is the reciprocal of the acquisition time $(T A)$ as is used before.

The most common methods are sliding window and keyhole techniques, both involving the view sharing strategy. The principles are first explained here, and the comparisons as well as the experimental results are presented in the next section.

\subsubsection{Sliding Window and MR Fluoroscopy}

The sliding window technique was originally proposed by Riederer in late 1980s to achieve a so-called MR fluoroscopy effect based on a Cartesian encoding scheme [19, 202, 203]. In this method, a "window" with a width equal to the desired number of phase encoding steps $N_{P E}$ is used to slide along the acquired data. Acquisitions within this window are used in the subsequent image reconstruction. The displacement between successive positions of the window is arbitrary, with a minimum of one $T R$ interval. Depending on the window shift (e.g. 
16 of total 256 phase-encoding steps), the image reconstruction rate is increased (e.g. 256/16= 16-fold), but the image acquisition is not affected. With such a repeated process, the contribution of any acquired phase encoding is observed much sooner in the reconstructed image after it is measured (e.g. 16.TR rather than 256.TR) and a continuous sequence of MR images can be observed when the MR data are being acquired.

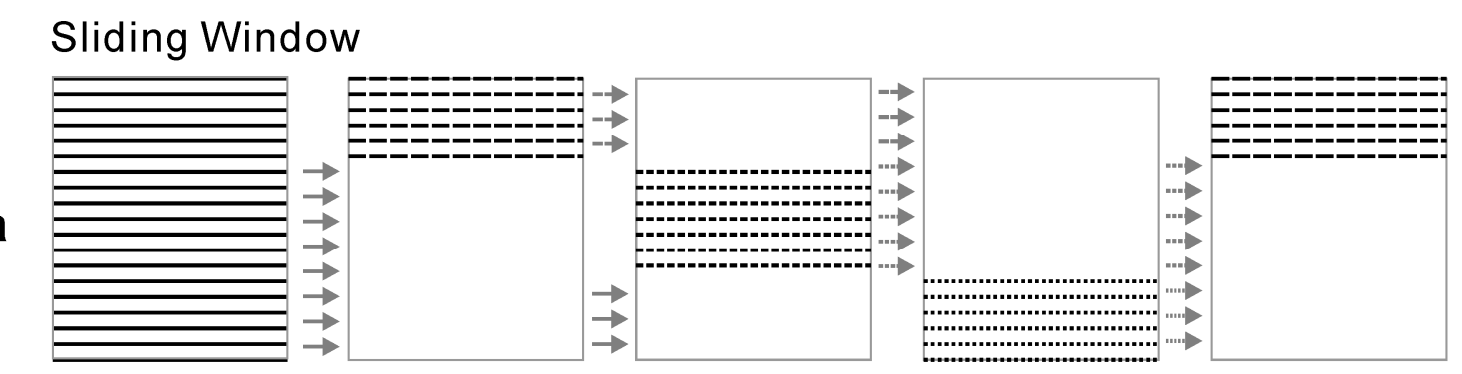

\section{Conventional Keyhole}

b

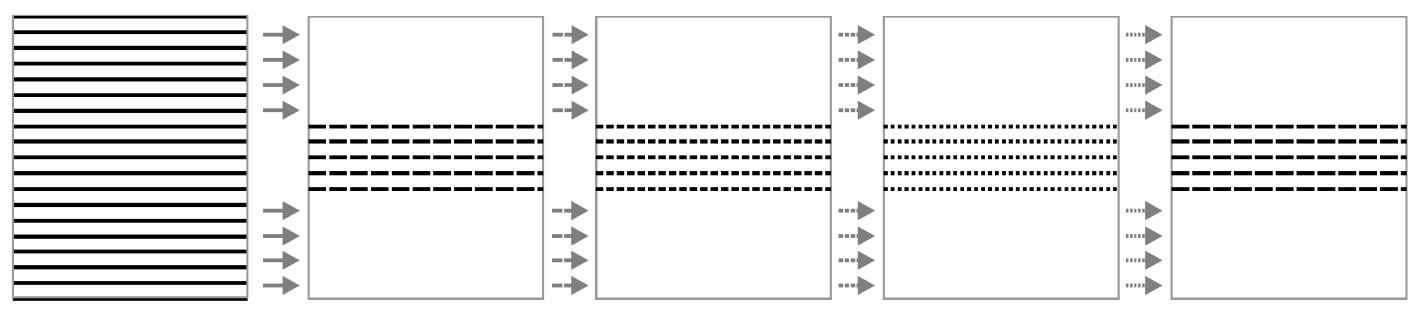

\section{Advanced Keyhole}

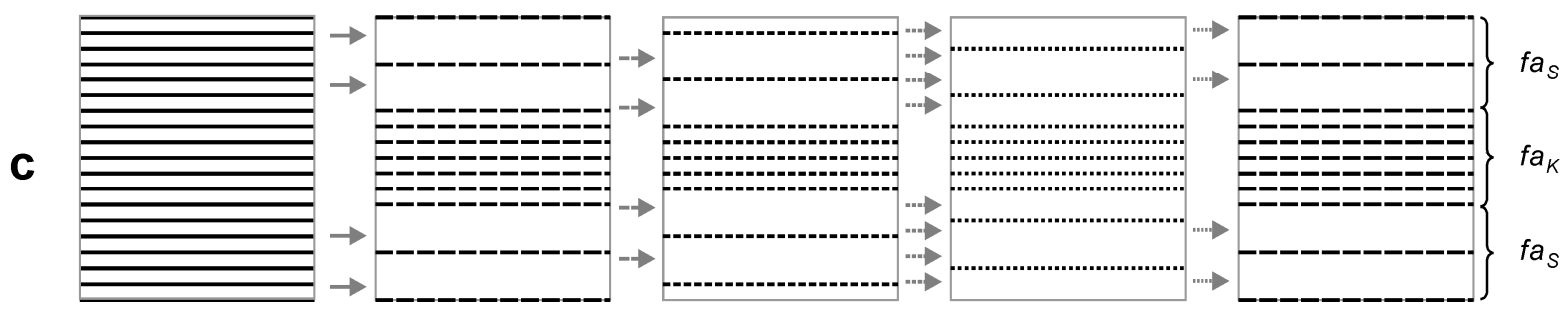

Figure 5.2 Schematic diagram of image reconstruction strategies for acceleration with (a) the sliding window, (b) conventional Cartesian keyhole, and (c) advanced Cartesian keyhole technique. The grey arrows indicate sharing of the data from previous measurements. $f a_{K}$ : keyhole factor; $f a_{S}$ : sparse factor.

More explicitly, whenever a new segment of the $k$-space is sampled, it replaces the corresponding old data which are acquired at the same positions. The new data is combined with the $k$-space data that are "shared" from the recent last acquisitions to form a complete data set, which is then transformed into an image, as illustrated in Fig 5.2a. Therefore, the sliding window technique is automatically integrated with view sharing strategy, where "view" stands for any frequency-encoding readout, either lines in Cartesian, interleaves in spiral, or spokes in radial encoding.

Ever since the concept of MR fluoroscopy came in existence, numerous developments and improvements have followed aiming at "real-time" MRI [204-209]. The advantage of this 
technique is a much higher frame rate without loss of spatial resolution, because the phaseencoding step, i.e. window width, is always the same for successive images. The disadvantage is that, because the spatial information is not equally covered by the rectilinear Cartesian lines, the low frequency components in the central $k$-space, which contribute more to the gross image contrast, are not always updated during the sequential acquisition of the segments. Thus, the motion of the object in the reconstructed images experiences jumps whenever the segment that corresponds to the $k$-space center is newly acquired and updated.

\subsubsection{Cartesian Keyhole Imaging}

In contrast to the sliding window method, the keyhole technique always measures the center of the $k$-space after the initial whole $k$-space measurement and during the following image acquisition, so that the changes of image contrast are better maintained. It was originally proposed in early 1990s for monitoring of the contrast agent administration [20, 21, 210]. As it is also based on Cartesian encoding scheme, the unmeasured $k$-space periphery, which contains high spatial frequency components and adds more to the image details such as object edges, can be copied from the initial measurement (Fig 5.2b), or can simply be zero-filled resulting in lower spatial resolution. The former approach is based on the assumption that the high spatial frequency content of the image is constant in time. Therefore, this technique is particularly suitable for dynamic contrast update without changes of the object outline and structure, for example, monitoring of exogenous contrast bolus uptake [20, 21], and also in 3D contrast-enhanced MR angiography by using the so-called TRICKS (time-resolved imaging of contrast kinetics) method as an extension of the 2D Cartesian Keyhole technique [211]. Such applications may also include monitoring of brain activities from diamagnetic deoxyhemoglobin as an endogenous contrast agent in fMRI [212, 213].

However, the disadvantage is also explicit: since the high frequencies are seldom or never acquired during the following measurements, the possible change of the object edge information is lost in successive image reconstructions [214]. For example, for dynamics, which involve structure changes or small contrast changes such as for interventional MRI during operation or biopsy, it is difficult to use this method to monitor the artificial needle insertion with good visualization of the needle tip position $[215,216]$. 


\subsubsection{Interleaved Radial Sliding Window}

The originally proposed sliding window and view sharing technique is combined with interleaved radial encoding scheme (i.e. reordered interleaving as described in Section 4.3.3) in this thesis for development of RT-MRI. The view sharing is performed after completion of each interleave of the radial views (i.e. each uniformly undersampled radial segment) in $k$ space. The image reconstruction and update rate is increased by a factor of $N_{\text {seg }}$, where $N_{\text {seg }}$ is the number of interleaves groups, as illustrated in Fig 5.3a.

\section{Interleaved Radial Sliding Window}

a

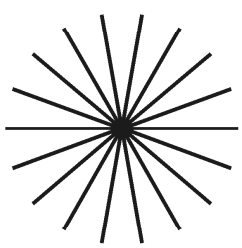

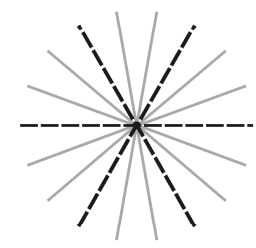
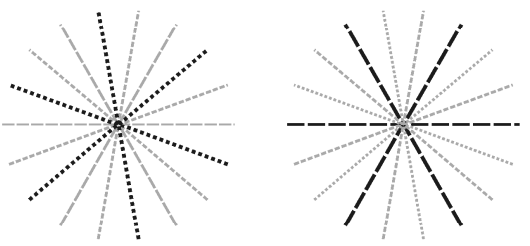

Linear Radial Sliding Window

b
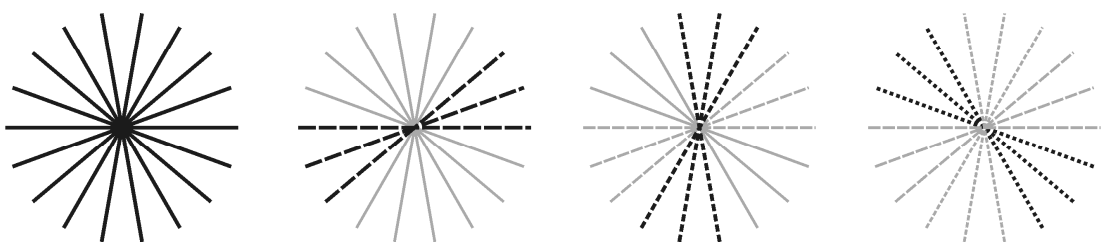

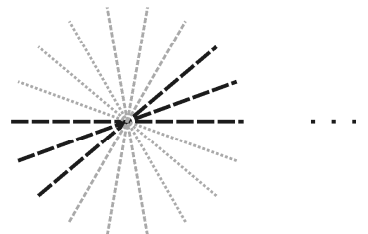

Figure 5.3 (a) Interleaved radial sliding window and (b) linear radial sliding window reconstruction. Dark views indicate data from the actual measurement and grey views indicate unmeasured data, which are shared from previous measurements. For simplicity, the example contains nine views $\left(N_{V}=9\right)$ with 3 interleaves $\left(N_{\text {seg }}=3\right)$ in a $180^{\circ}$ encoding scheme.

Because of the unique sampling patterns, each radial view covers equal spatial information containing both low and high frequency components. The unwanted jumps observed with the Cartesian sliding window disappear with continuous update during dynamic imaging. Nevertheless, an advanced Cartesian-based keyhole reconstruction strategy combined with sliding window and view sharing technique (termed advanced keyhole for simplicity) as illustrated in Fig 5.2c, a linear radial sliding window technique as illustrated in Fig 5.3b, and an advanced radial keyhole technique as illustrated in Fig 5.7, were developed and implemented, respectively, for a thorough experimental comparison with the proposed interleaved radial sliding window reconstruction. The strategies and the experimental results are described and discussed in Section 5.3.2, after a short review of available reconstruction methods. 


\subsection{Comparison of Available Reconstruction Strategies}

\subsubsection{Brief Review}

\section{Reconstruction with Non-Cartesian Encoding}

Other than FBP and gridding methods, a few more reconstruction strategies exist that transform non-Cartesian data to images, which include both FT-based [217-223] and non-FT approaches [224-226]. Discussions of each individual strategy are beyond the scope of this dissertation, but in short, all these methods face more or less similar problems, either difficulties in parametrization [219, 225, 226], intensive computational burdens [218, 220, 223], or complicated technical and mathematical implementations, for which even the acquisition and encoding scheme needs to be changed [224, 227]. Thus it is of no doubt that they pose serious limitations for RT-MRI uses, at least with current computer techniques. Therefore, the convolution gridding, which has been proved accurate enough [221], was employed in this thesis for the reconstruction of non-Cartesian data.

\section{Reconstruction with Undersampling for Acceleration}

In addition to sliding window and keyhole techniques, many other strategies have been proposed to further accelerate the image reconstruction rate by reducing the amount of the data that are normally required (undersampling) without significantly compromising the image quality. The shortening of the data acquisition and the undersampling pattern is controlled by exploiting the amount of redundancy within the data, while the proper reconstruction is realized by applying dedicated algorithms based on the assumption that the dynamics of natural objects exhibit a high degree of correlations in $k$-space (spatial-frequency domain) and time (temporal domain). As such strategies vary, the brief review and comparison here is not meant to list and to be comprehensive for all possible methods, but to address the reason why the straightforward sliding window technique was used in this thesis.

First of all, strategies exploiting correlations within $k$-space include partial Fourier (PF) $[18,76,77]$ and parallel imaging (PAT) methods [22-25, 228], and also some other techniques such as reduced-FOV methods [229, 230]. Noticeably, the rapid development of PAT within the last decade has enabled considerable imaging time reduction. PAT works in either image domain after Fourier transformation $[23,24,228]$ or $k$-space domain before Fourier 
transformation $[22,25]$. Both approaches rely on multiple coil sensitivity profiles to recover the unmeasured data points. Since the number of encoding lines is reduced, it also helps to eliminate artifacts caused by long readout such as in EPI or to reduce SAR deposition such as in FSE and TrueFISP. But reduced signal acquisition also inevitably leads to a decrease in the SNR, which together with the effectivity of the applied algorithms, limit the acceleration factor that can be achieved. This category may even include non-Cartesian sampling with variable density, such as the radial scheme, since the interpolation of the $k$-space grids is based on the principle that each $k$-space point contains correlated information about other points (depending upon the convolution window function in this case) from one time frame independent to the other time frames.

Second, strategies based on temporal correlations with no dependence on other spatial points mainly include the sliding window and keyhole techniques. The recovery of the unmeasured data points at one time frame through data sharing - considered interpolation or extrapolation - is from other time frames, no matter from the nearest neighbor (i.e. in sliding window) or from the much earlier measurement (i.e. in keyhole). Some similar methods use more sophisticated interpolators or modeling but basically rely on the same principle [231233]. Noticeably, to trade for undersampling with acquisition reduction, the UNFOLD (unaliasing by Fourier-encoding the overlaps using the temporal dimension) method purposely eliminates half of the encoding lines and creates typical aliasing artifacts in an alternating manner by shifting the sampling pattern every other time frame. Thus, the aliased component can be removed from the desired (non-aliased) component by applying a low-pass filter along the temporal axis [234, 235]. However, the proper selection of the FOV to avoid the true dynamic object or dynamic areas of the object overlapping in the replications (i.e. FOV at least as large as the dynamic region) and to ensure two components encoded at different locations in the temporal frequency domain restrict the undersampling factor. In addition, the difficulty to choose proper parameters for the filter (e.g. filter width) also limits the use in practice. Furthermore, the approach to apply filter along temporal axis implies the need to acquire a series of data before image reconstruction, which is not feasible for realtime imaging. Although certain improvement was suggested for RT-MRI, the reconstruction scheme was only implemented off-line with a limited frame rate [236].

Third, strategies trying to utilize correlations in both $k$-space and time $(k-t)$ [231], are a combination of the two above approaches. The typical example is the so-called $k-t$ BLAST (broad-use linear acquisition speed-up technique) or $k$ - $t$ SENSE [237]. The techniques improve the estimation of the unmeasured data by incorporating a set of low spatial frequency 
training data combined with a temporally averaged image over the whole acquisition stage, which are sparsely sampled. The proposal of this strategy was followed by a couple of similar techniques [238-242] that all exploit the temporal correlations based on the UNFOLD method and the spatial correlations based on the PAT, each with certain individual improvements in the reconstruction algorithms. Although these Cartesian-based techniques are compatible with non-Cartesian schemes including radial encoding [243], the extended reconstruction time due to much more intensive computation hinders it from immediate update and display. A highly undersampled radial scheme with $k$ - $t$ reconstruction termed HYPR (highly constrained backprojection) was proposed particularly for MR angiography [244]. This FBP-based technique is able to reconstruct images with good quality from only 10 radial views per frame (corresponding to about 100-fold undersampling in azimuthal direction). However, similar to $k$ - $t$ BLAST, the requirement to combine all of the acquired data to obtain a time-averaged "composite image" for reduction of undersampling streaking artifacts and increase of SNR excludes HYPR from being applied for prospective data transformation. Although it is possible to employ progressive updated composite image with reduced average window and thus shortened reconstruction delay, it is limited, similar to 2D keyhole or 3D TRICKS [211] techniques, to the situations in which the object edges and structure information remain stationary during acquisition (e.g. vessel position), while only the rapidly changing contrast is involved (e.g. blood flow, first-pass contrast bolus, diffusion, ECG-gated myocardial motion) [191, 245, 246].

Although the methods differ significantly in their ability to recovery or compensate the unmeasured data, for image reconstruction strategies with highly undersampled data in general, 1) averaging approaches (e.g. HYPR, $k$ - $t$ BLAST) are not suitable for dynamics involving many structural changes such as during swallowing or speaking; 2) reference or base-image approaches can lead to artifacts and false signal representation due to misregistration (e.g. keyhole, RIGR) or spatial coil sensitivity miscalculation (e.g. SENSE-based methods) such as during musculoskeletal kinetic studies; 3) prior knowledge or training data approaches (e.g. UNFOLD, $k$ - $t$ BLAST, feature-recognizing MRI [217]) are limited and may fail when the assumption is not met. As these strategies are proposed to address specific questions for particular applications, they are not able to provide a generic solution for realtime imaging of dynamic physiological processes. In addition, the much longer time required for image reconstruction, despite the accelerated acquisition due to large undersampling factor, is not able to achieve true real-time data processing and online image update, which includes the recently proposed compressed sensing (CS) concept [247-250]. Therefore, the original 
sliding window technique with view sharing strategy was implemented in this thesis in combination with interleaved radial FLASH for the development of RT-MRI.

\subsubsection{Experimental Comparison}

\section{Interleaved Radial Sliding Window vs. Advanced Cartesian Keyhole}

In order to compensate for the drawback of the conventional Cartesian keyhole imaging, a different approach was implemented combining the sliding window and view sharing technique. It frequently acquires the high frequency component in a homogeneously incremented way. As shown in Fig 5.2c, the central $k$-space area is always acquired and the exact number of lines in this area is controlled by the keyhole factor $f a_{K}$. Meanwhile, the peripheral $k$-space area is acquired in a homogeneously interleaved manner and is updated in an incremented way. The number of lines in this area is controlled by the sparse factor $f a_{S}$. In such a way, both high and low frequency components are frequently acquired and updated, and acceleration can be flexibly achieved by selecting different combination of $f a_{K}$ and $f a_{S}$. Extensive experiments have been done to test the feasibility of this advanced Cartesian keyhole reconstruction. Fig 5.4 present experimental results of monitoring volunteer's head rotation. Images in Fig 5.4 a, b and c were acquired by using the advanced Cartesian keyhole reconstruction, while images in $\mathrm{d}$ were acquired by using the interleaved radial sampling in combination of sliding window reconstruction. The three chosen frames in each row were three subsequent images during head rotation. For comparison, the speed of the movement was controlled by the visual stimuli and was thus almot identical for each experiment. The imaging slice was selected across the cerebrospinal fluids (CSF) and sinus, to mimic the extreme case of motion, which involves changes from both contrast and structures, and with large amplitude following an irregular track. Each image was composed of 128 phaseencoding lines in advanced Cartesian keyhole cases and of 135 views in interleaved radial sliding window case with 5 interleaves (i.e. 5 accelerations). FOVs were all $192 \times 192 \mathrm{~mm}^{2}$ yielding $1.5 \times 1.5 \mathrm{~mm}^{2}$ in-plane resolution with all other parameters identical for both methods (TR/TE 5.3/2.6 ms, FA 15 $B W 810 \mathrm{~Hz} / \mathrm{Pixel}$, SLT $6 \mathrm{~mm})$. A 12-channel head coil (Siemens AG, Erlangen, Germany) was used in all studies.

In the first examination, only $1 / 10$ of the $k$-space in the middle $\left(f a_{K}=1 / 10\right)$ and every $20^{\text {th }}$ line in the periphery $\left(f a_{S}=20\right)$ were always measured and continuously updated during dynamic imaging. That is to say, instead of 128 lines, only 18 lines (12 lines in the middle and 
6 lines in the periphery on both sides) were acquired for every new image frame. Thus, a 7fold acceleration was achieved. However, the old contrast and edge information from the large data set, which was previously measured, overlapped with the current information, which was represented by only a small portion of data. This resulted in severe artifacts in the reconstructed images as shown in Fig 5.4a. In the second examination, the middle part was increased to half of the $k$-space (i.e. $128 / 2=64$ lines) with $f a_{K}=1 / 2$, so that the gross contrast was better maintained - noticed from the CSF and fat contained scalp with high intensity. But the edge residual due to still less updated high frequency component remained dominant, as shown in Fig 5.4b. In order to further improve the image quality, based on the second examination, every other line in the $k$-space periphery was continuously (alternatively in this case) updated in the third examination. Noting the position of the sinus, this indeed showed better representation of the motion, but nevertheless, obvious ghosting artifacts were still present in most of the images as shown in Fig 5.4c and the acceleration that could be achieved was limited in this case to 1.3 -fold $(T U \approx 500 \mathrm{~ms}, 2 \mathrm{fps})$. In the last examination shown in Fig $5.4 \mathrm{~d}$, with unique radial encoding characteristics as discussed before, and with further combination of sliding window reconstruction, much smoother update of the moving object with 5-fold acceleration ( $T U \approx 143 \mathrm{~ms}, 7 \mathrm{fps}$ ) was achieved. Only mild undersampling and motion induced streaks were present in some of the images.

The results clearly show that, for Cartesian keyhole methods, it is very difficult to choose adequate parameters of how much should be measured in the middle and in the periphery of the $k$-space, respectively, in order to achieve smooth update of the dynamic changes with satisfactory image quality. Second, although such advanced scheme has been applied for monitoring contrast agent uptake in dynamic contrast enhanced MRI (DCE-MRI) [251] and for investigation of brain activities in fMRI [252] combined with EPI acquisition, it is, however, similar to conventional Cartesian keyhole, very difficult to observe structural changes, which are inevitably involved in most of the physiological dynamic processes (joint movements, swallowing, speaking, etc.). As a matter of fact, it is very sensitive to such gross motions, during which the phase and amplitude discontinuities can induce severe ghosting artifacts, as demonstrated here. 

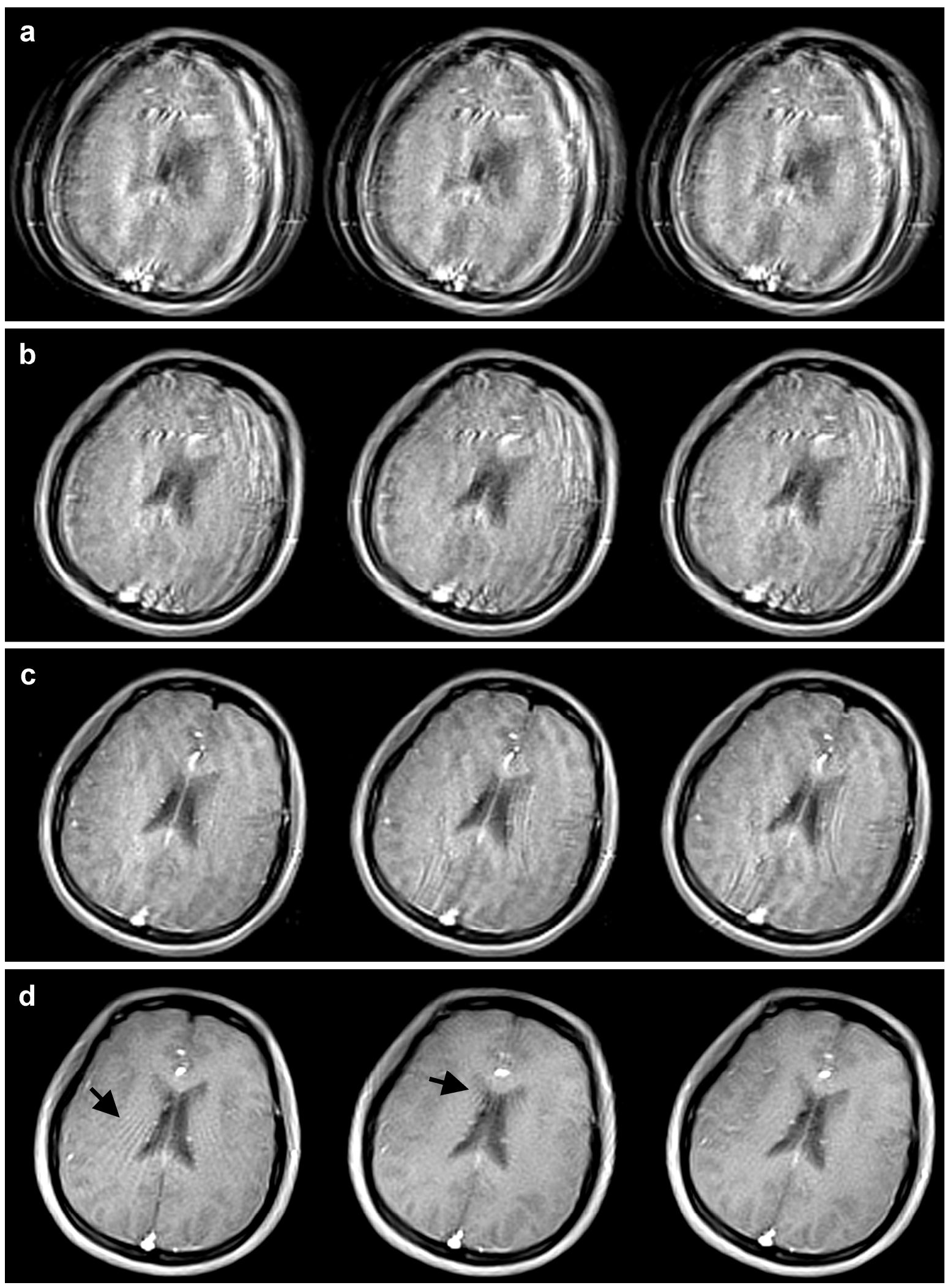

Figure 5.4 Comparison of $(\mathbf{a}, \mathbf{b}, \mathbf{c})$ Cartesian keyhole and (d) the interleaved radial sliding window technique for observing head rotation in real time. The frames represent three sequntial images from each examination, during which keyhole factor $\left(f a_{K}\right)$ and sparse factor $\left(f a_{S}\right)$ in $(\mathbf{a}, \mathbf{b}$, and $\mathbf{c})$ were $1 / 10$ and 20,1/2 and 20,1/2 and 2, respectively. Images in (d) were acquired with 135 views for a complete image data set and 5-fold acceleration with 5 interleaves. Arrows indicate slight streaking artifacts caused by undersampling. 


\section{Interleaved Radial Sliding Window vs. Linear Radial Sliding Window}

Although it has been demonstrated in the last chapter that the interleaved radial encoding scheme offers better image quality concerning motion robustness than the linear radial encoding scheme, for better understanding of the properties of sliding window technique in combination with radial encoding, two sampling and reconstruction schemes were compared here - besides the proposed interleaved radial sliding window technique, the linear radial sliding window reconstruction was implemented as illustrated in Fig 5.3b. The experimental results from a cardiac experiment in a transversal plane are shown in Fig 5.5. For linear radial sliding window, dynamic changes of the object, which are normally simultaneous in all directions during the acquisition of an entire $k$-space data are only covered over a certain angular segment. Such update pattern results in loss of information over a wide angular range and causes strong degradation of the robustness of radial imaging [183, 185, 253, 254], and the motion appears discontinuous and asynchronous, as demonstrated in Fig 5.5a.
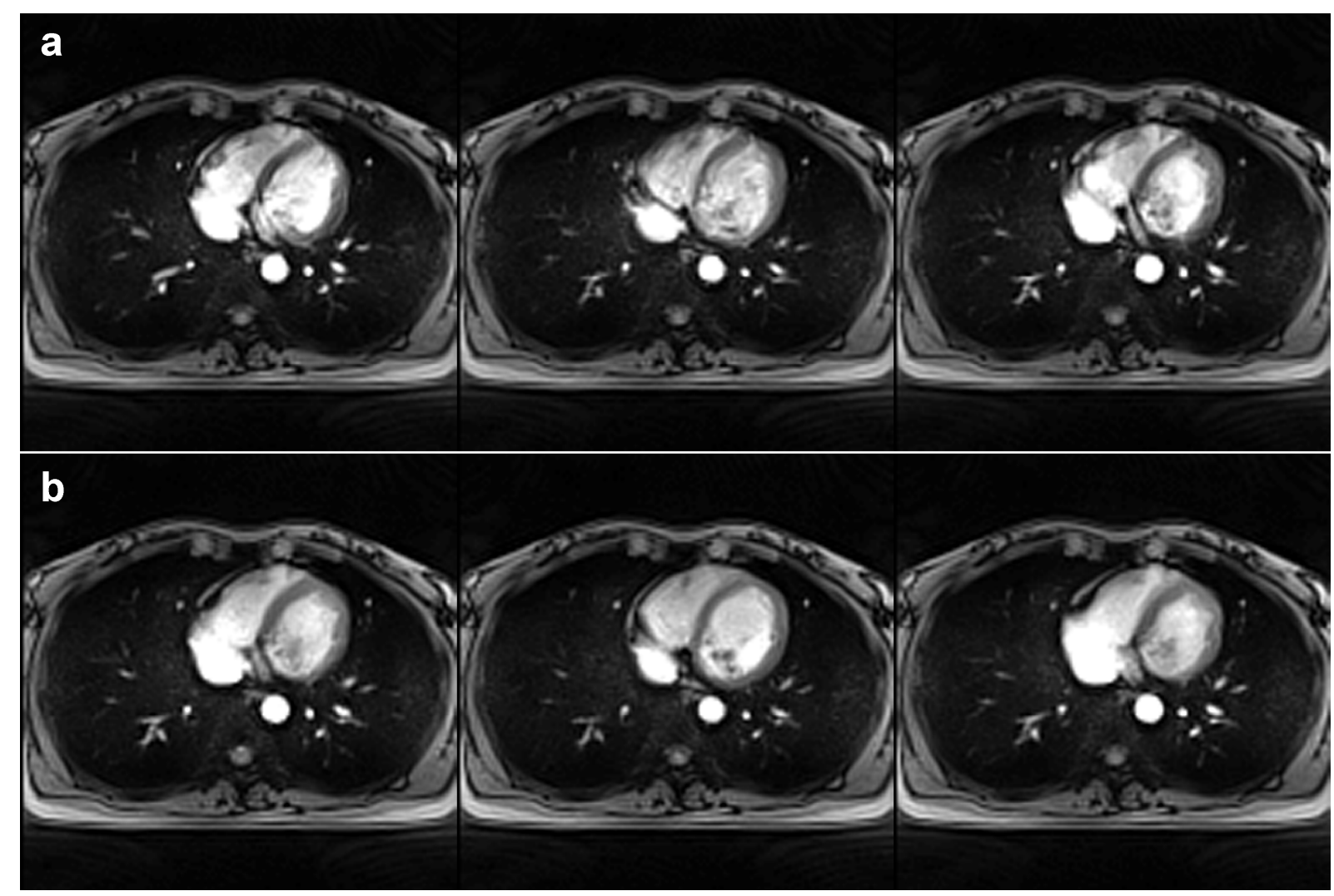

Figure 5.5 Comparison of (a) the linear and (b) the interleaved radial sliding window technique. The frames in each row represent three consecutive images from one cardiac series at diastole, with free breathing and without ECG synchronization. Note the discontinuous intensities in (a). Imaging parameters: spoiled radial FLASH, 16-channel body matrix coil (Siemens AG, Erlangen, Germany), FOV 256×256 $\mathrm{mm}^{2}$, matrix size $128 \times 128$, in-plane resolution $2.0 \times 2.0 \mathrm{~mm}^{2}, S L T 8 \mathrm{~mm}, T R / T E 2.02 / 1.3 \mathrm{~ms}, F A 8^{\circ}, B W$ $1950 \mathrm{~Hz} /$ Pixel, 205 views with 5 interleaves, i.e. 5 linear segments in (a) and 5 interleaved segments in (b), effective temporal resolution $12 \mathrm{fps}$. 
As for interleaved radial sliding window, because the $k$-space is continuously updated in all directions, the motion appears to be smooth during the imaging process [254], as seen in Fig 5.5b. However, because each interleaved segment is an undersampled data set (or more precisely, sub-data set), the desired segment number should not be too large. Otherwise, increased streaking artifacts may be easily induced by discrepancies between segments due to severe undersampling [28], as demonstrated from head rotation experiments in Fig 5.6. It should be noted that, first, such streaks were far less obvious in cardiac imaging as shown in Fig $5.5 \mathrm{~b}$, because the gross motion in this case concentrated in the middle of the FOV with a relatively limited area, not like the head rotation case with a large movement extent. Second, although such a desired segment number puts a limit on the acceleration rate that sliding window can achieve, in fact, it is proved to be sufficient to have a moderate segment number (sliding window acceleration factor). This will be discussed in detail in the next chapter.
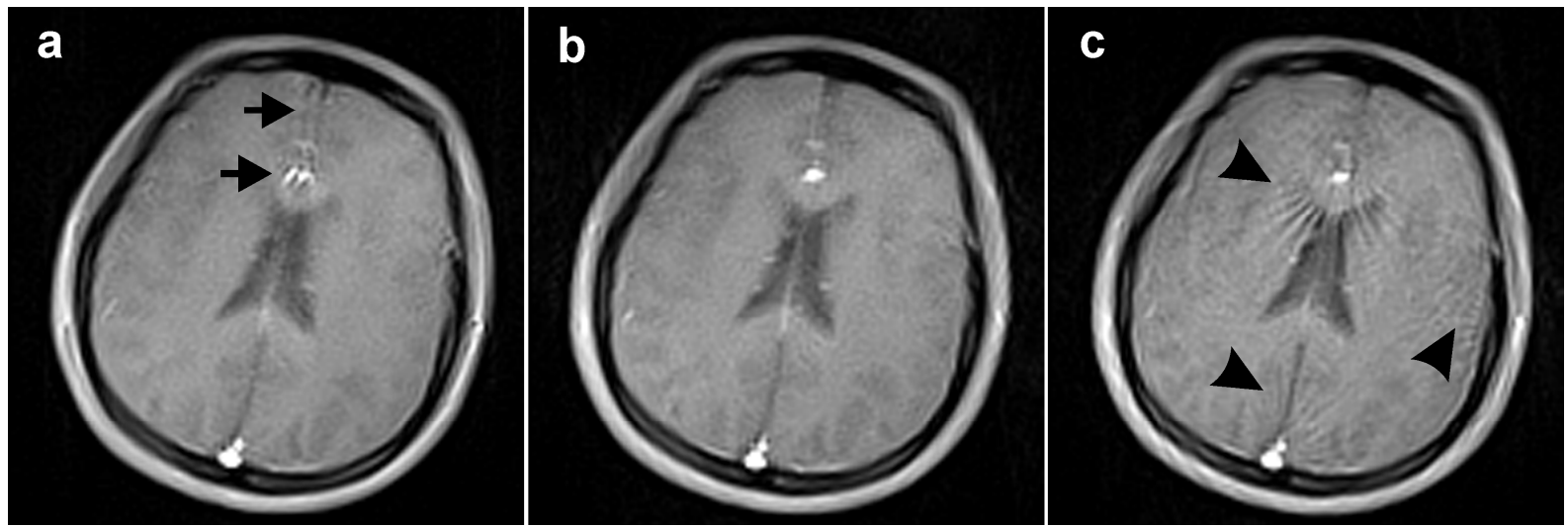

Figure 5.6 Influence of the number of interleaves on the radial sliding window reconstruction for a head rotation study. Experimental parameters were the same as that in Fig 5.4d except for 195 views. The images in (a, b, c) were acquired with 1 (i.e. no acceleration), 5, and 13 interleaves, respectively. Note the discontinuity (arrows) in (a) and increased streaks (arrow heads) in (c).

\section{Interleaved Radial Sliding Window vs. Advanced Radial Keyhole}

The interleaved radial sliding window technique is further compared with an advanced radial keyhole reconstruction. The latter was originally suggested for contrast weighted imaging with FSE acquisition, so-called $k$-space weighted image contrast (KWIC) [255] method. The oversampling in the central $k$-space is exploited to enhance the contribution of views that are acquired at specific $T E$ in the FSE echo train. And by weighting views with different $T E$, respectively, multiple T2-weighted images can be reconstructed from a single image data set. It was later combined with an interleaved scheme [188] for dynamic contrast-enhancement studies of breast lesions using gradient echo acquisition and contrast agent injection [256]. 


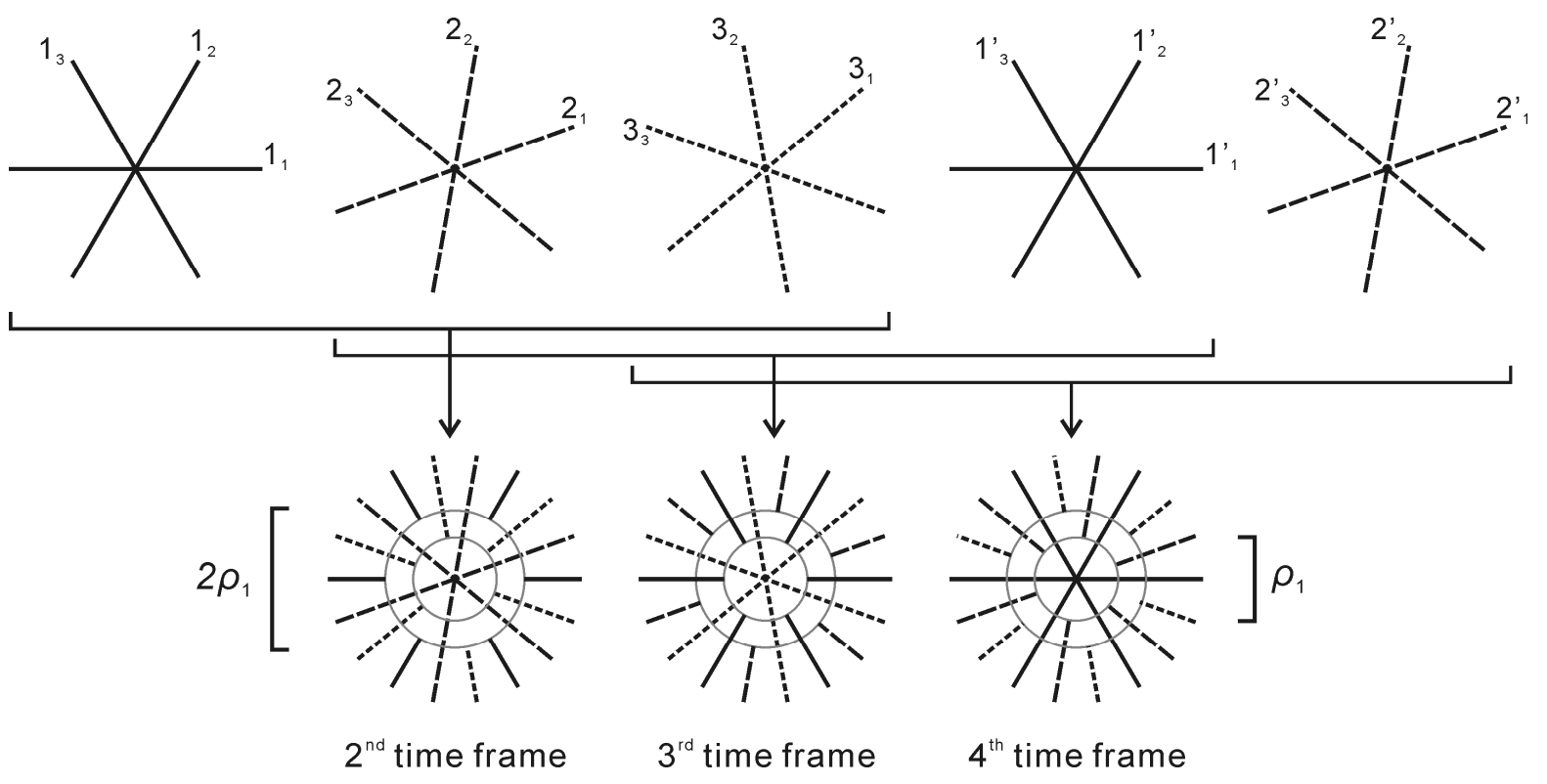

Figure 5.7 Schematic diagram of the advanced radial keyhole technique. The algorithm was implemented in the MRISim software [128] to compare the image quality with that of the interleaved radial sliding window technique from the same data set.

Similarly, for the dynamic physiological processes, it was expected that, by adapting the same strategy - weighting the chosen segment in the central $k$-space region - to constrain the contrast change during motion to the correspondingly weighted segment instead of the complete data set and thus to improve the temporal fidelity. Furthermore, rather than combining the current segment with all previously acquired ones to yield the current image frame, it was also expected to represent the motion more faithfully if the segments that were acquired afterwards were combined as well. The implemented reconstruction algorithm for offline post-processing is illustrated in Fig 5.7. It is based on the sliding window technique, but the two differences are: first, for each time frame during the dynamic study, only the data from the segment of interest was used in the central $k$-space region (e.g. views of $3_{1}, 3_{2}$ and $3_{3}$ in the $3^{\text {rd }}$ segment). Depending on number of views within the segment, a distance parameter was calculated to determine the radius from the $k$-space center to meet the Nyquist sampling criterion according to Eq. 4.4:

$$
\rho_{1}=\frac{\Delta k \cdot N_{V}}{\pi \cdot N_{s e g}}
$$

Thus, data from other segments were set to zero within this circle (Nyquist circle) so that only the segment of interest contributed to the contrast in the corresponding image (e.g. $3^{\text {rd }}$ time frame). To avoid sudden change in signal intensity, which would lead to artifacts in the image, 

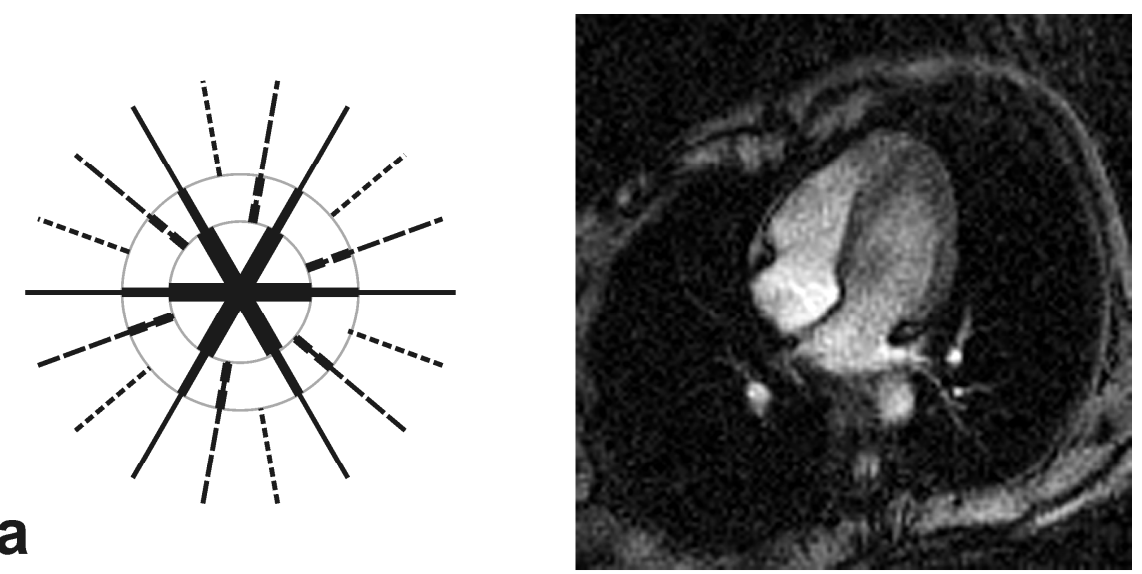

a

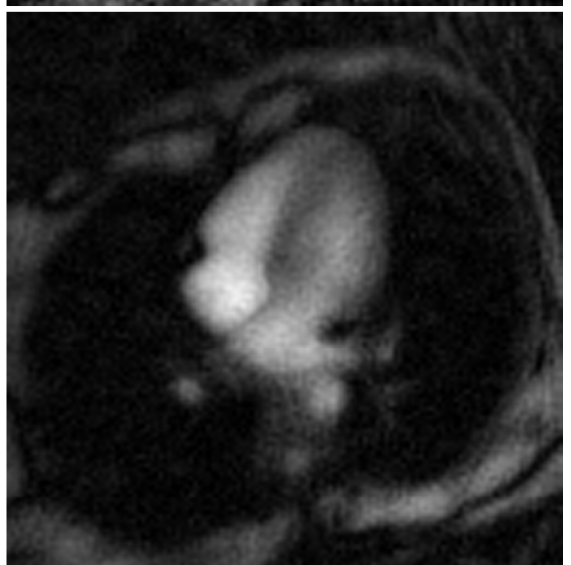

b
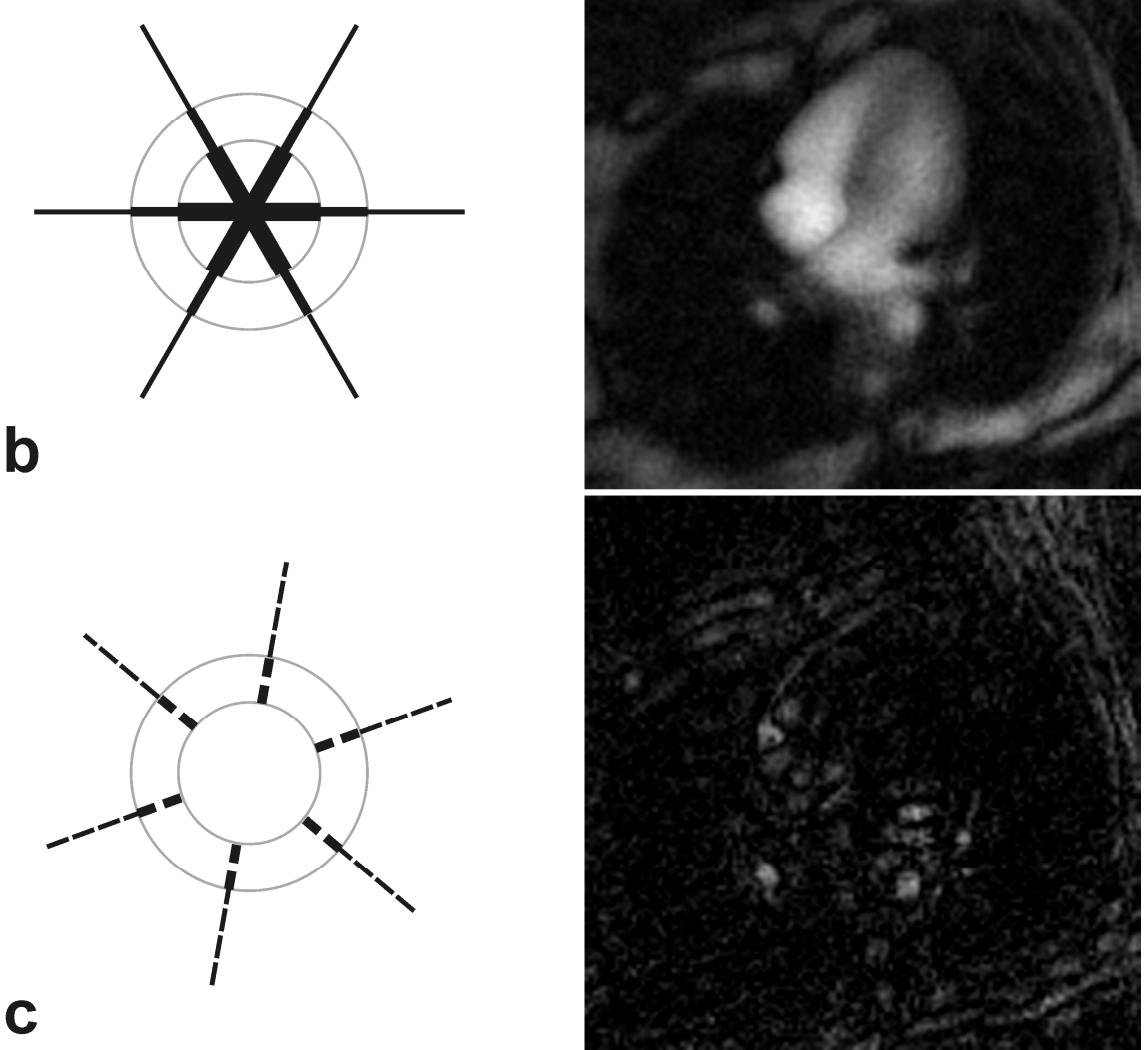

C

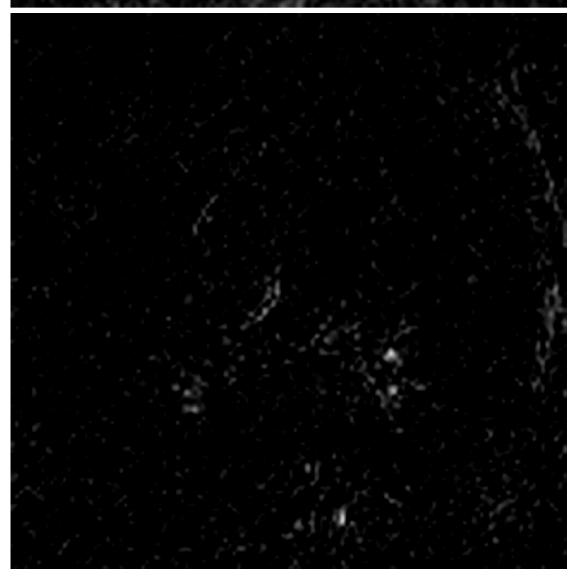

Figure 5.8 Advanced radial keyhole technique applied to a cardiac data set in an anatomically defined 4chamber view. Images from (a to d) on the right side show reconstructed results using different parts of the acquired data as illustrated. The line thickness indicates the weighting of each segment, while the gray circles indicate the calculated Nyquist circles. 
a second circle with a radius of $2 \rho_{1}$ was determined, and the sampling density compensation was also changed correspondingly as suggested in [255]. Second, once the current segment (i.e. segment of interest) was acquired, the corresponding image was not immediately reconstructed by combining with previous segments (e.g. segment 1,2 and 3 for $3^{\text {rd }}$ time frame). Instead, it did not take place until the next segment was acquired and then included for the reconstruction of the current time frame by replacing the oldest one (e.g. segment 2, 3 and 1 ' for $3^{\text {rd }}$ time frame). In such a manner, the reconstruction buffer contains a mixture of data from both the previous and following measurements, more information regarding temporal process was involved to represent individual time frame. Of course a short reconstruction delay due to waiting for the next segment was introduced which equaled $T R \cdot\left(N_{V} / N_{\text {seg }}\right)$. This implemented strategy was termed here advanced radial keyhole.

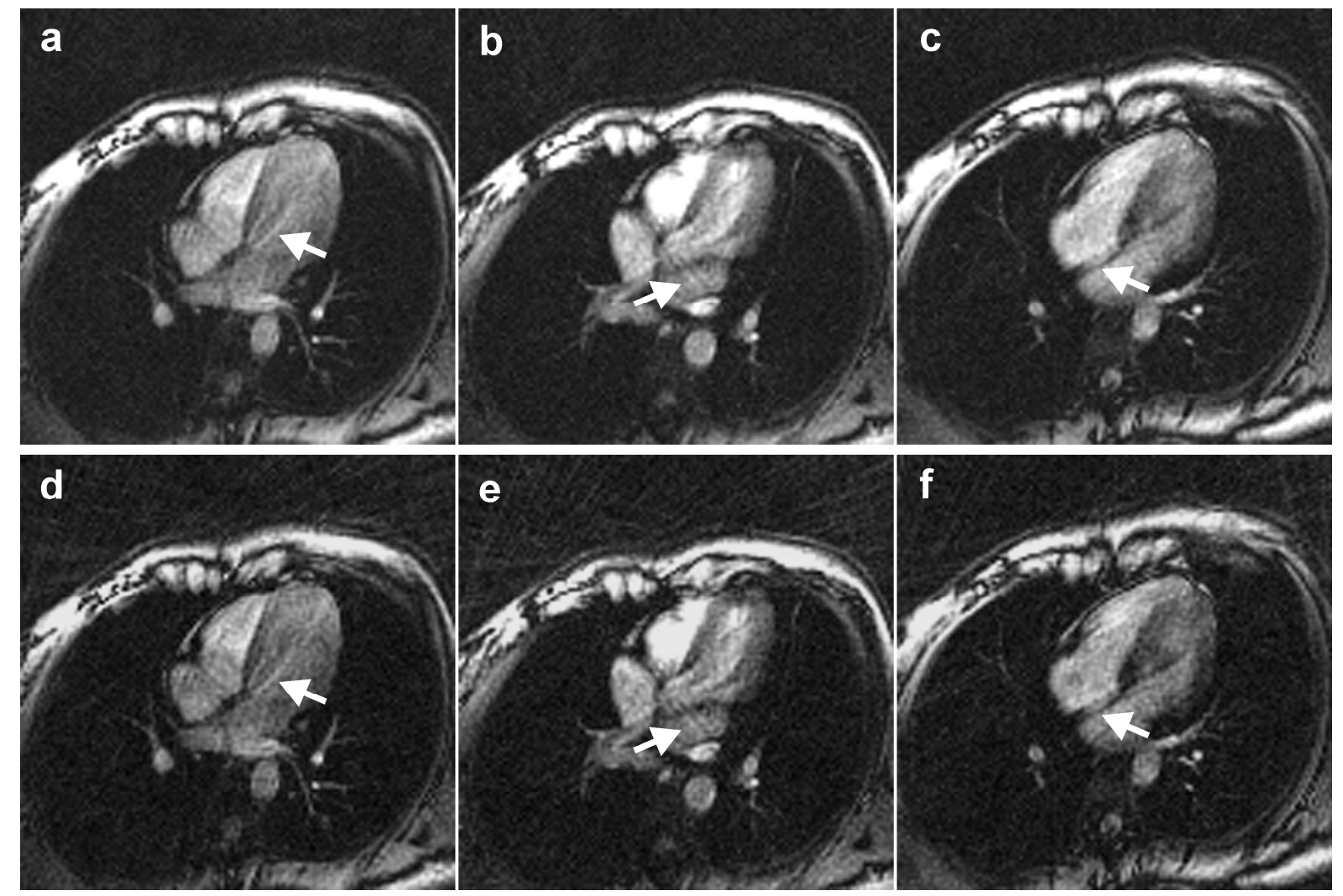

Figure 5.9 Comparison of (a-c) the interleaved radial sliding window and (d-f) the advanced radial keyhole reconstruction. The imaging parameters: spoiled radial FLASH, 16-channel body matrix coil (Siemens AG, Erlangen, Germany), FOV $256 \times 256 \mathrm{~mm}^{2}$, matrix size $128 \times 128$, in-plane resolution $2.0 \times 2.0 \mathrm{~mm}^{2}, S L T 8$ mm, 85 views with 5 segments, TR/TE 2.02/1.3 ms, FA $8^{\circ}, B W 1950 \mathrm{~Hz} /$ Pixel. Arrows indicate smearing and streaking artifacts, which were induced by motion inconsistencies.

One example from the validation studies of this reconstruction strategy is shown in Fig 5.8 using images from a cardiac RT-MRI measurement. Five segments were used during the radial sampling and 85 views were employed for a complete image data set. For offline 
reconstruction with in vivo data, corresponding $\rho_{3}=3 \rho_{1}$ and $\rho_{4}=4 \rho_{1}$ were applied. For simplicity, only three segments were illustrated in the figure. Fig 5.8a was reconstructed from a complete image data set in advanced radial keyhole manner. Fig 5.8b was reconstructed only from the main segment (i.e. segment of interest, with $85 / 5=17$ views shown as straight lines). The bold lines within the first circle in the central $k$-space region represent proper density weighting to avoid abrupt intensity chances. Fig 5.8c and Fig 5.8d were reconstructed from adjacently acquired segments - before (dashed lines) and after (dotted lines) the segment of interest. Note that the gross image contrast was mainly contributed by the segment of interest which was expected to more faithfully represent the motion changes at corresponding time point, while the image details were contributed from the neighboring segments acquired which was expected to include more temporal information.

A comparison between the straightforward radial sliding window reconstruction and the advanced radial keyhole reconstruction is shown in Fig 5.9, by applying both of these two strategies to the same cardiac experiment data. The acquired data were from a healthy volunteer (female, 35 years old) during free breathing and without ECG synchronization. The imaging parameters were the same as in Fig 5.8, with a 16-channel body coil (Siemens AG, Erlangen, Germany). Images with the straightforward radial sliding window method (a to c) were obtained directly from real-time online reconstruction, while those with the advanced radial keyhole method ( $\mathrm{d}$ to $\mathrm{f}$ ) were obtained from offline reconstruction. For the latter, although the segment of interest was weighted in the central $k$-space region to be more dominant in the image contrast, and although more information regarding the temporal process was included in each reconstructed time frame, the smearing caused by sharing of the data at different time points during rapid object motion, for example, at cardiac wall and septum (arrows), was not improved as expected. In addition, weighting of particular segment and zero-setting of the others led to loss of the advantage of oversampling in the central $k$ space, although the Nyquist criterion was fulfilled. This caused a decrease in the SNR and more streaking artifacts in the resultant images. Therefore, the straightforward radial sliding window method, as proposed, is concluded to be superior to the advanced radial keyhole method. 


\subsection{Summary}

Fourier transformation is the most commonly used reconstruction method that can be efficiently applied when the $k$-space data is sampled in a rectilinear fashion with phase- and frequency-encoded acquisitions, as described in Section 2.2.2. But for radial imaging with non-gridded positions and varying sampling densities, specific procedures are required. These include: 1) density correction by density compensation function; 2) convolution gridding by interpolation (i.e. Kaiser-Bessel window function); 3) 2D inverse fast Fourier transformation; 4) roll-off correction and image cropping.

In order to further increase temporal resolution beyond the physical (e.g. gradient strength and slew rate) and physiological (e.g. nerve stimulation) constraints, image reconstruction approach uses the strategy of updating, rather than waiting to refill, the $k$-space image reconstruction buffer, so that fast computing hardware can reconstruct and display images (from the mixture of image data in the buffer) in real time without delay of the standard acquire-delay-reconstruct-display paradigm. The sliding window with view sharing technique was implemented for interleaved radial FLASH sequences, which allows for reconstruction update periods shorter than the acquisition time of a full dataset and achieves better temporal resolution for the development of RT-MRI.

The effectiveness was demonstrated by comparing it to three other advanced approaches: 1) the advance Cartesian keyhole reconstruction shares the motion sensitivity with Cartesian encoding scheme and is similar to conventional keyhole technique, in which a smooth update of structural changes is difficult to achieve; 2) the linear radial sliding window reconstruction has continuous update of only a part of $k$-space with limited angle and direction, and thus cannot faithfully represent the motion to be imaged; 3) the advanced radial keyhole suffers from decreased SNR and increased streaking artifacts due to the loss of oversampling in the central $k$-space region.

The proposed RT-MRI technology was implemented at a high field (3T) human MR system, which will be described in the next chapter. The practical realization regarding the achievable spatial and temporal resolution will also be discussed. Furthermore, specific results from individual applications of potential clinical or biomedical interest will be given in Chapter 6. 


\section{Chapter 6}

\section{Realization of Real-time MRI on 3T Human MRI System}

Based on the methodological development of RT-MRI described in the previous chapters, this chapter focuses on the practical realization of the proposed method in a real environment. Implementation work has been done on a conventional clinical MRI system with a magnetic field strength of 3T, as described in Section 2.4. High field imaging is attractive, because it has the potential to improve the SNR due to increased polarization of spins [257]. With proper pulse sequence design, the effects of increased susceptibility and off-resonance resonance effects can be managed, and the SNR increase can be used to improve spatial resolution and/or temporal resolution. The image quality and contrasts for RT-MRI as well as the achievable spatial and temporal resolution are experimentally evaluated in this chapter.

\subsection{Radial FLASH - Pulse Sequence Design}

\subsubsection{Pulse Sequence Design}

The implemented radial FLASH sequences are illustrated in Fig 6.1, with gradient waveforms as described in Eq 4.1 to achieve radial $k$-space trajectories. Directly after the RF excitation in the presence of the slice selection gradient, spins are in different precessing phases. This decreases the amplitude of the total signal. To maintain phase coherence, a reverse (refocusing) gradient, i.e. with negative polarity for compensation, is applied after the slice-selection gradient. Generally, the RF pulse occurs at the center of the slice-selection gradient and dephasing occurs only during the second half of the gradient. Ideally then, the refocusing gradient has only half of the duration (at the same strength) of the slice-selection gradient. Starting from this point, the phase of all spins within the selected 2D slice is equal, which corresponds to the center position in the 2D $k$-space, where $k_{x}=k_{y}=0\left(t_{1}\right.$ in Fig 4.1). The 
dephasing (sometimes also called prephasing) gradient in the frequency-encoding direction $G_{x}$ causes the magnetization to be dephased and this corresponds to a movement away from the $k$-space center in the $k_{x}$ direction. At the same time, the switching of the phase-encoding gradient in the orthogonal direction $G_{y}$ corresponds to a shift in the $k_{y}$ direction. Thus in conjunction, they cause a shift from the $k$-space center $\left(t_{1}\right)$ to the outmost $k$-space margin $\left(t_{2}\right.$ in Fig 4.1) and the strength and length of them determine the $k$-space location, as described in Section 2.2. Up to this point in the measurement, the magnetization has been properly prepared. A single line (radial view) of the $k$-space is then sampled by switching the readout gradients under the presence of both $G_{x}$ and $G_{y}$, which cause the magnetization to rephase. This corresponds to a constant movement in the opposite direction towards the $k$-space center.

Spoiled

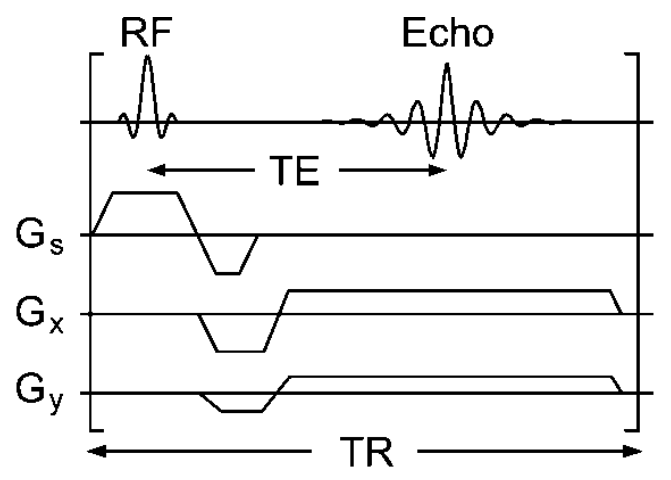

Refocused

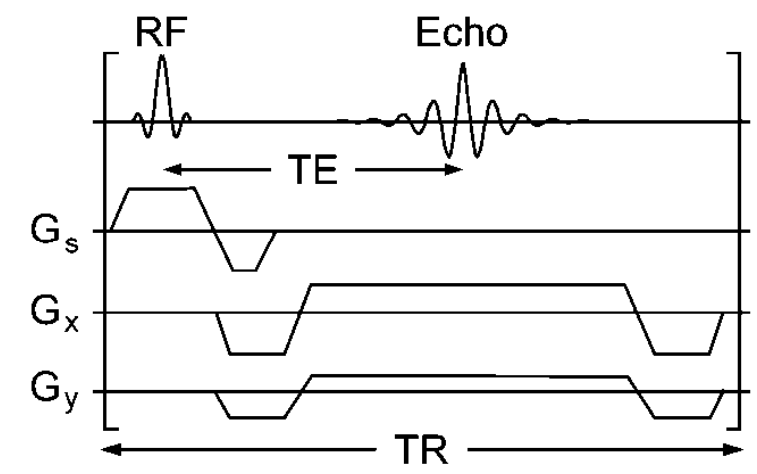

Balanced

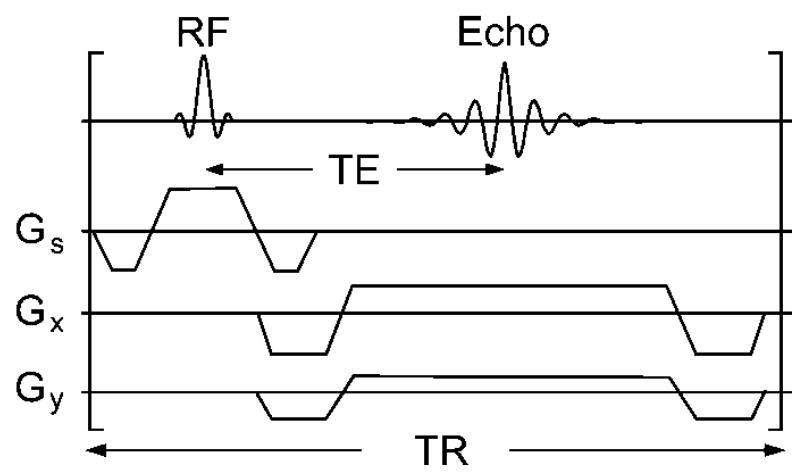

Figure 6.1 Sequence diagram of spoiled, refocused and balanced radial FLASH RT-MRI techniques. 
A complete rephasing of the spins results in an echo signal when the momentum of the first gradient is exactly cancelled by that of the second one ( $t_{3}$ in Fig 4.1). The continuing application of $G_{x}$ results in a further dephasing of the spins ( $t_{4}$ in Fig 4.1) until finally they are spoiled or rewinded (rephased). For spoiled FLASH, RF spoiling with incremented pulse phase of $50^{\circ}$ is used to destroy the residual transverse magnetization. On the contrary, to achieve refocusing effect, a gradient of the same strength but of opposite polarity is applied after data acquisition and before the next RF excitation pulse, to incorporate residual transverse magnetization directly into the refocused SSFP (Fig 6.1). In refocused FLASH, the next phase per $T R$ is constant. Further, to realize balanced condition, which has zero phase per $T R$, a dephasing/ prephasing gradient along the slice selection direction with the same strength and direction as the slice selection refocusing gradient is applied before each RF excitation (Fig 6.1), so that the gradient effects undergone by spins are fully balanced. In such a manner, through repetition of gradient switching with varied amplitude, all trajectories across the $k$ space center are sampled.

\subsubsection{Image Contrasts}

Examples demonstrating the obtainable image contrast from all three FLASH variants are presented in Fig 6.2. Images were acquired from inanimate phantoms with aqueous solutions of different paramagnetic ion concentrations, as well as from the brain in a transversal plane, the head and the knee in a middle sagittal plane, and the heart in an anatomically defined short-axis plane. For comparison, all images with the same object were acquired with an identical slice and with comparable imaging parameters (short $T R$ and $T E$, high $B W$, low $F A$ for spoiled FLASH and relatively higher $F A$ for refocused and balanced FLASH, $360^{\circ} k$-space coverage with odd number of views, with trajectory corrections applied). The phantom, brain, head and knee images were taken during static experiments, while the heart images were taken from a real-time dynamic study during free breathing of the subject and without ECG synchronization. The mean signal intensity for SNR was calculated from white matter in the brain image and blood of the left ventricle (white arrow head) in the heart image. The CNR was calculated from the mean signal intensity difference between white matter and CSF in the brain image, or from blood and septum in the heart image. The ROIs for signal amplitude and noise measurements were all $50 \mathrm{~mm}^{2}$. 

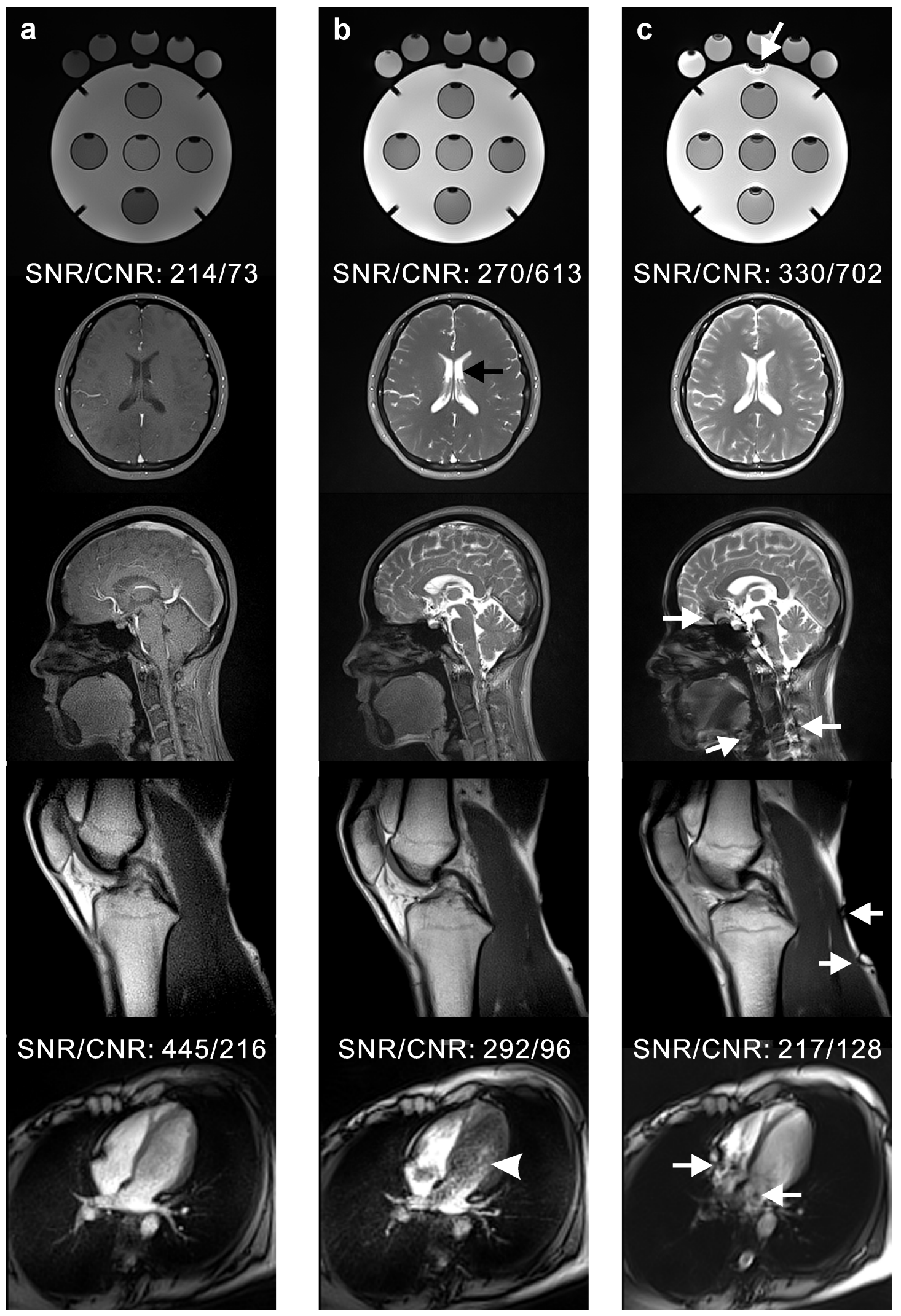

Figure 6.2 Experimental results of (a) spoiled, (b) refocused, and (c) balanced radial FLASH RT-MRI for a phantom and different parts of the human body: the brain in a transversal and a middle sagittal plane, the knee in a middle sagittal plane, and the heart in an anatomically defined 4-chamber plane. Black arrow indicates the contrast offered by refocused FLASH due to T1/T2-weighting; white arrow head indicates distortion of the SSFP signal due to motion in refocused FLASH; white arrows indicate the susceptibility artifacts in balanced FLASH. 
Spoiled FLASH showed typical T1 weighted contrast (Fig 6.2a) - low signal intensities from long T1 tissues (e.g. CSF, muscles) with dark area in the images, and high signal intensities from moving fluids (e.g. small vessels in the brain and blood in the heart), described as inflow effect in the subchapter 3.1.

For refocused FLASH at relatively larger $F A$ s and short $T R$ s (i.e. $T R \ll \mathrm{T} 2$ ), the signal contrast is related to T1/T2-weighting, causing structures with long T2, such as CSF, to become bright (black arrow). Generally, as a matter of fact, any kind of phase instability from one repetition interval to the next, e.g. the rapid movement of the heart, precludes the generation of transverse coherences and does not establish an SSFP signal [57], which yields motion-related distortion in the image (white arrow head in Fig 6.2b).

The balanced case shows the highest SNR and CNR, e.g. in the static brain image, as expected. However, because of the artifacts and distortions (white arrows), this technique is fundamentally restricted in practice by several factors. First, the high FAs (normally between $50^{\circ}-90^{\circ}$ ) may easily exceed the SAR limits, particularly at the high field of $3 \mathrm{~T}$, although certain reduction approaches have been proposed [258, 259]. Second, more critically, the ideal steady state is disturbed by small local differences in precession frequency, which cause small deviations from the ideal case of zero net phase at the end of each TR. When the phase angle $\theta$ approaches $180^{\circ}$, there is a rapid loss of the steady state and a decrease in signal. Therefore, areas of imperfect magnet uniformity or susceptibility changes in the tissue can cause the offresonance precession angle $\theta$ to approach $180^{\circ}$ and finally introduce dark stripes - "banding artifacts" in the image. This becomes a fundamental problem at high fields as the offresonance effects increase linearly with field strength, although principally it is possible to reduce such problems by keeping $T R$ s so short that less time is given for dephasing $\theta$ to develop [66, 67]. Noticeably, at low fields up to $1.5 \mathrm{~T}$, similar artifacts due to concomitant gradient field effects [260] are independent of $T R \mathrm{~s}$, because the phase accrual occurs only when the gradients are active, and therefore will not be weakened by shortening the TRs alone [261]. Other remedies to overcome the banding artifacts are either to simulate different imaging frequencies during a few preparation scans before the real measurement in order to determine the central frequency, so-called frequency scout imaging [262], or to apply subjectrelated fine shimming by using local shim coils and control software before the real measurement. However, results from both previous studies [263, 264] and the presented work (Fig 6.2) have shown that these measures are still insufficient to produce artifact-free images. Especially during real-time imaging which aims to monitor dynamic processes, the movement of the object through areas of different local magnetic field strengths dramatically abates the 
effect from such approach, which results in difficulties in interpretation of valuable functional or diagnostic information, as shown in Fig 6.3. Let alone in, for example, advanced cardiovascular real-time study, such adjustment process (normally on the order of tens of seconds) due to need of interactive imaging orientation change during fast heart movement and irregular flow will predictably interrupt the whole imaging process. Therefore, the balanced version is discarded for the purposes of this thesis, and the developed RT-MRI sequences use relatively low $F A s$ in the range of $5^{\circ}$ to $30^{\circ}$.
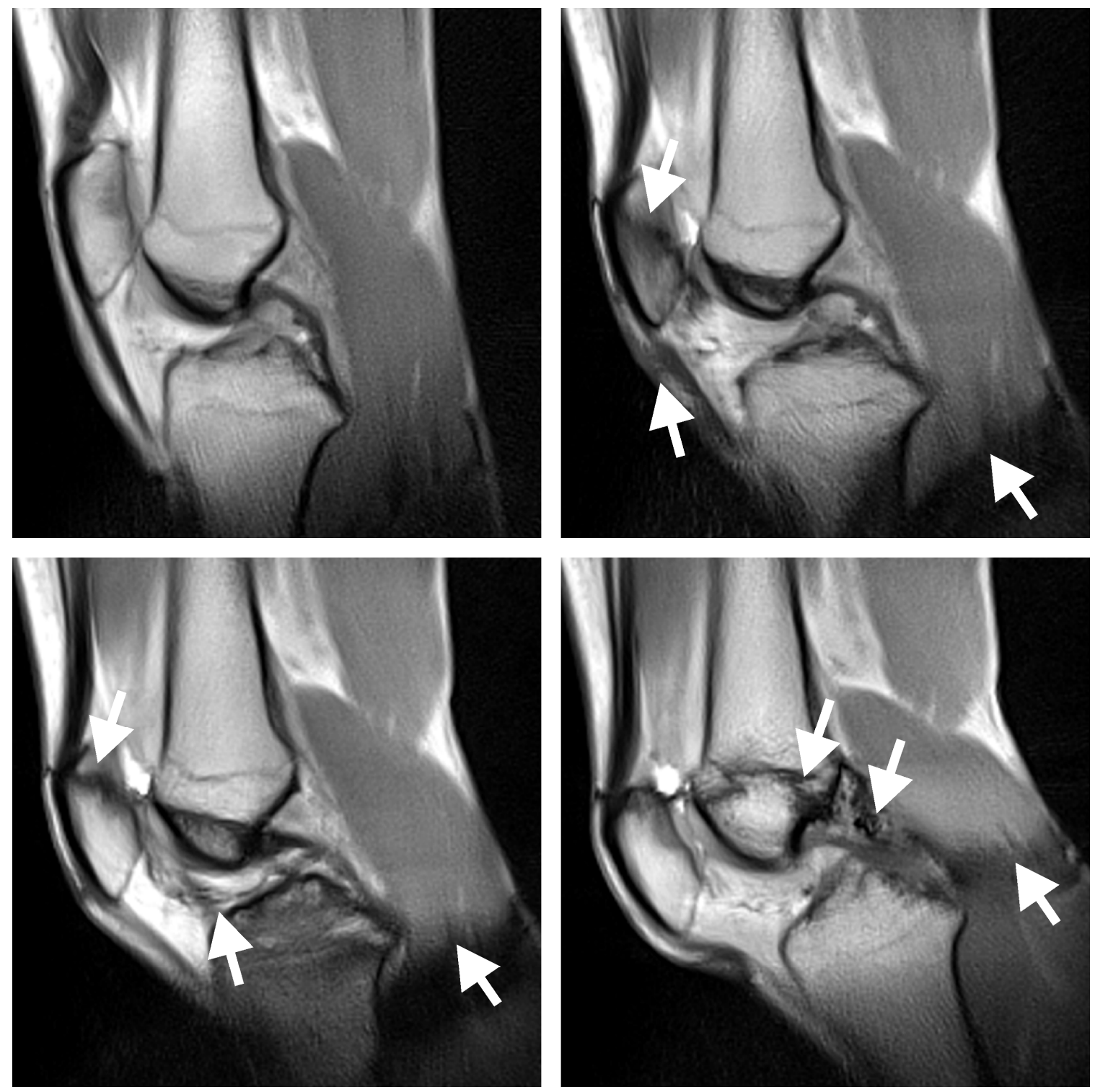

Figure 6.3 Banding artifacts from balanced SSFP (TrueFISP) during angulation of the tibiofemoral joint. The four frames (from upper left to lower right) were chosen from a sequence of radial real-time series during voluntary knee flexion. The first frame (upper left) was taken before the start of the movement with proper magnetic field homogeneity, and is free of artifacts. During the movement, the motion-induced susceptibility changes caused a failure of the previous shimming. Strong banding artifacts occurred, which were also moving (white arrow) with the motion. 

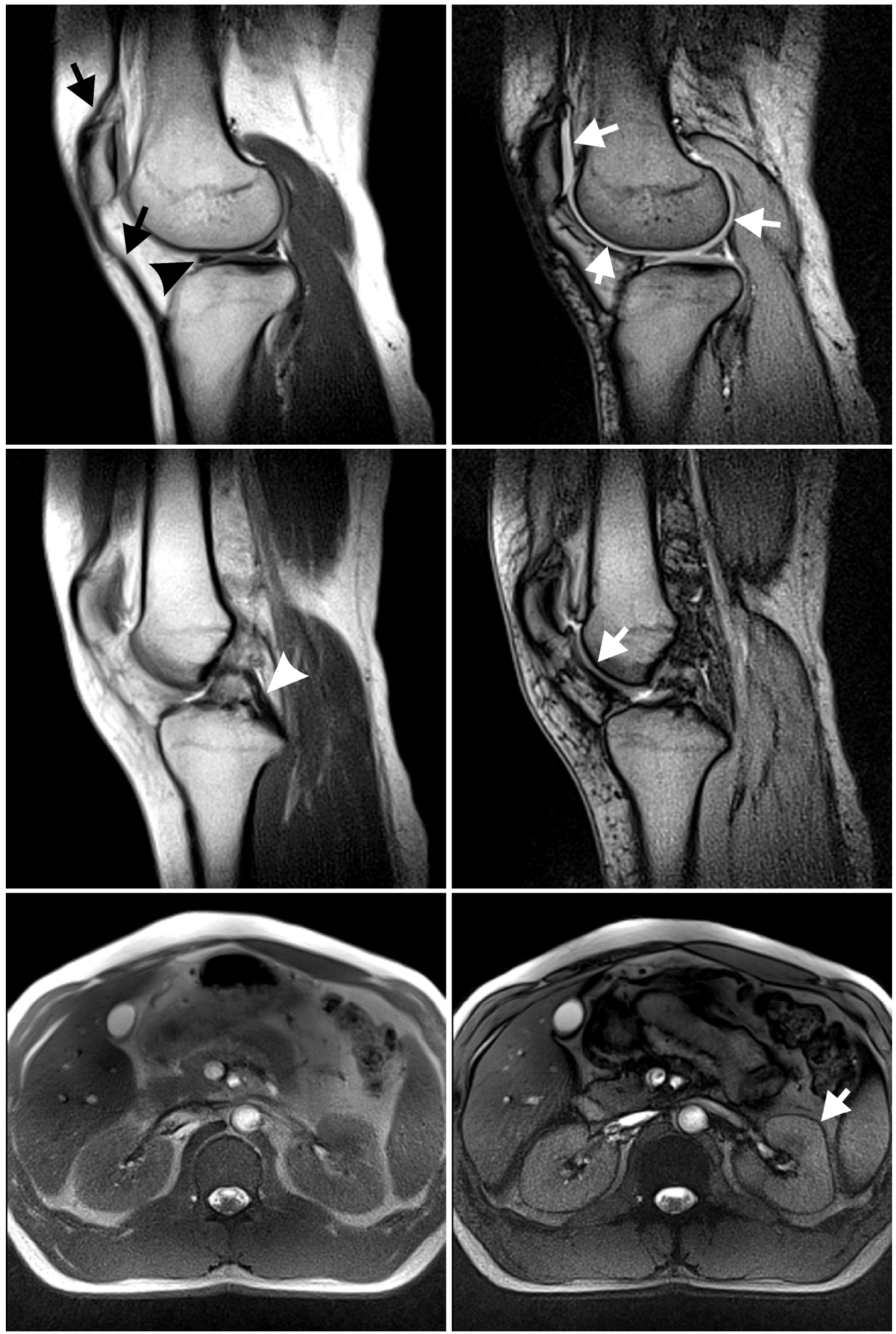

Figure 6.4 In-phase (left) and opposed-phase (right) contrast provided by radial FLASH RT-MRI. Images of the knee in lateral-sagittal plane (top), mid-sagittal plane (middle) and abdomen in a transversal plane (bottom) were acquired by refocused radial FLASH with $T E$ at $2.5 \mathrm{~ms}$ for in-phase contrast and at $3.5 \mathrm{~ms}$ for opposed-phase contrast. Patellar tendons (black arrow), cruciate ligaments (white arrow head), meniscus (black arrow head) are better displayed in in-phase images, whereas the articular cartilage and the border of viscera such as kidneys (white arrows) are better delineated in opposed-phase images. 
In addition, because of the chemical shift of fat and water (as described in Section 3.3.2), i.e. slightly different precession frequencies and thus different phases, their transverse vectors fall in and out of phase periodically. Therefore, for voxels in which fat and water co-exist, specific $T E$ s can be selected to offer in-phase contrast with stronger signal or opposed-phase contrast with partially cancelled signal (Fig 6.4). As the clear visualization of the water contents may facilitate the diagnosis of pathology, such quasi-suppression of fat signals is often very helpful. For example, in musculoskeletal and abdominal imaging as shown in Fig 6.4, patellar tendons, cruciate ligaments, meniscus were better displayed in in-phase images. On the contrary, due to partially cancelled signal of fat and water, articular cartilage and the border of viscera such as kidneys were better delineated in opposed-phase images.

The implemented radial FLASH real-time MRI pulse sequences and algorithms were integrated to the manufacture defined software framework and structure (Siemens AG, Erlangen, Germany) under C++ (v6.0: Microsoft Inc., Redmond, WA) environment. The workflow chart is shown in Appendix A.

\subsection{Radial FLASH - Image Quality}

Experimental comparisons that are given in Chapter 5 demonstrate the effectiveness of the proposed RT-MRI technology. Here the image quality and the achievable spatial and temporal resolution are further discussed.

Extensive in vivo tests have shown that normally three to six-fold acceleration (i.e. three to six radial interleaves) with 30 to $300 \mathrm{~ms}$ update time and correspondingly 3 to $30 \mathrm{fps}$ effective temporal resolution are efficiently enough to resolve most of the physiological changes. This makes the proposed method highly flexible in selecting different spatial and temporal resolutions. For example, for joint movements or interventional processes (e.g. biopsy needles and catheters insertion), which are relatively slow and controllable but involve motions of small structures, an effective temporal resolution of 3-10 fps with higher spatial resolution is suitable for online monitoring and functional evaluation. On the other hand, for cardiovascular motions or swallowing processes, which are rapid and involuntary, higher temporal resolution of at least $20 \mathrm{fps}$ is desirable. Fig 6.5 is an example of using moderate spatial resolution $\left(2.0 \times 2.0 \times 8.0 \mathrm{~mm}^{3}\right)$ and high temporal resolution $(20 \mathrm{fps})$ in thoracic and upper abdominal imaging. 125 views were used with 5 interleaves, and $T R / T E=2.02 / 1.3 \mathrm{~ms}$. Multiple motions such as from rapid heartbeat, blood flow in large (e.g. aorta) and small 
vessels (e.g. in lung), periodic movement of liver and chest wall, are involved. However, the artifact-free images with good contrast demonstrate the high ablity of this proposed method in dynamic imaging of time-varying objects in real time. More examples of achievable spatial and temporal resolution and its high adaptability to motions are given below.
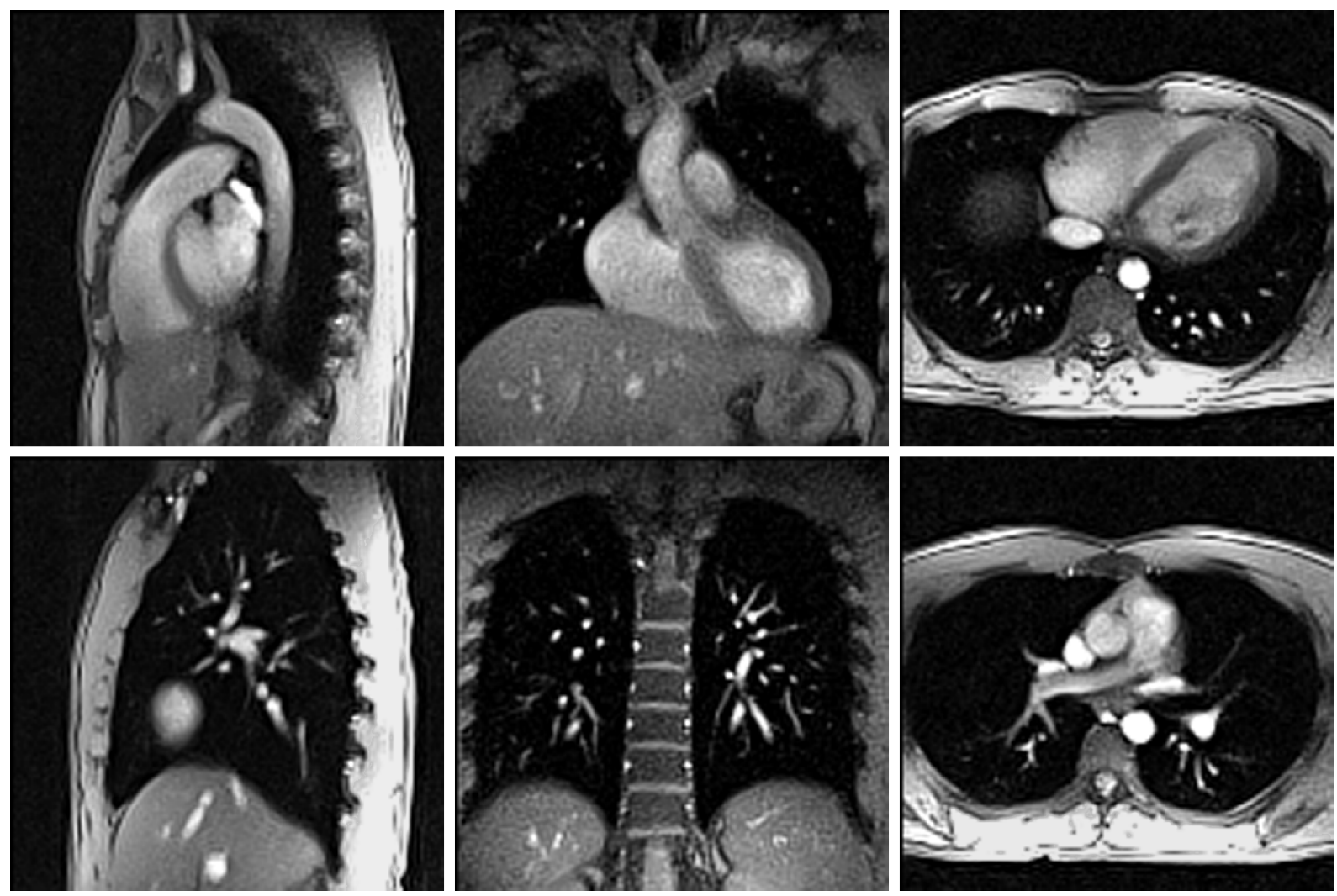

Figure 6.5 Radial FLASH RT-MRI of thorax and upper abdomen in sagittal (left), coronal (middle) and transversal (right) planes. Spatial resolution $2.0 \times 2.0 \times 8.0 \mathrm{~mm}^{3}$. Such images may serve as localizer scans.

\subsubsection{Spatial Resolution}

Fig 6.6 shows an example that increases the spatial resolution by a larger image matrix size and smaller slice thickness, while decreasing the view number to maintain the same temporal resolution. All images were acquired with the same FOV $\left(256 \times 256 \mathrm{~mm}^{2}\right)$ during a real-time study of the cardiac motion with free breathing and without ECG synchronization by using a 32-channel body coil (Siemens AG, Erlangen, Germany). Images at both systolic and diastolic phases from an anatomic short-axis plane were chosen for demonstration.

Fig $6.6 \mathrm{a}$ and $\mathrm{b}$ were acquired with an image matrix size of $128 \times 128$ and a slice thickness of $8 \mathrm{~mm}$, which resulted in a spatial resolution (voxel size) of $2.0 \times 2.0 \times 8.0 \mathrm{~mm}^{3}$. Together with 125 views, 5 interleaves for sliding window reconstruction, and $T R / T E=2.02 / 1.30 \mathrm{~ms}$, an 
effective temporal resolution of $20 \mathrm{fps}(T U=50 \mathrm{~ms})$ was achieved. At such an imaging speed and image resolution, the myocardial contraction and thickening during the cardiac cycle can be very well resolved and represented, and the purpose of online monitoring and functional evaluation can be achieved. In Fig $6.6 \mathrm{c}$ and d, by acquiring with a larger matrix size of $160 \times 160$ and smaller slice thickness of $6 \mathrm{~mm}$, a smaller voxel size of $1.6 \times 1.6 \times 6.0 \mathrm{~mm}^{3}-\mathrm{a}$ typical spatial resolution for clinical diagnostic purpose - was reached. Together with 115 views, 5 accelerations, and $T R / T E=2.20 / 1.40 \mathrm{~ms}$, the same temporal resolution of $20 \mathrm{fps}$ was maintained. Decrease of SNR can be observed in $\mathrm{c}$ and d due to smaller voxel size, but all main tissue structures remain clear. In future, more dedicated receive coils should be able to further enhance the SNR.

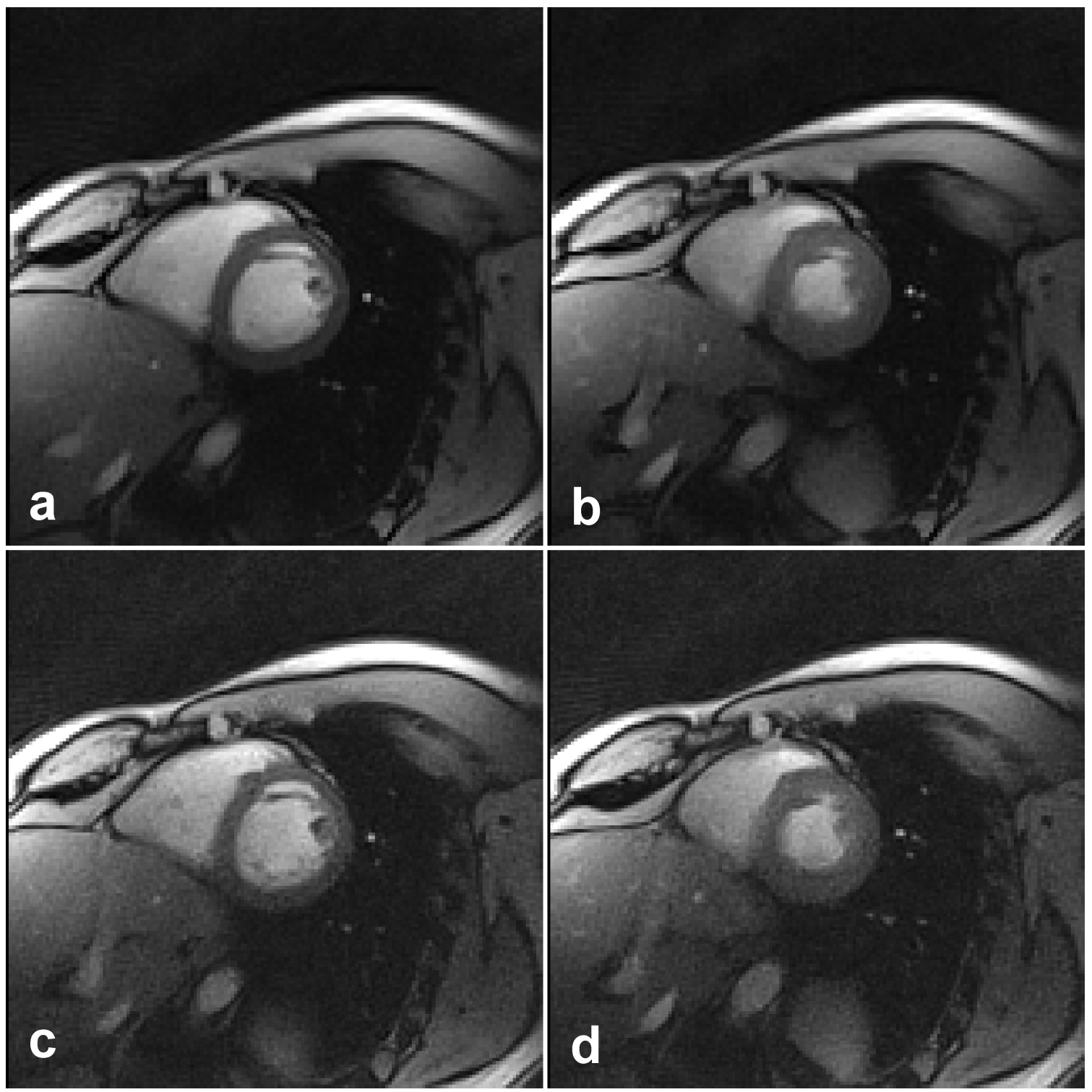

Figure 6.6 Radial FLASH RT-MRI of the human heart with different spatial resolution and 20 fps temporal resolution (short-axis view). (a) and (b) $2.0 \times 2.0 \times 8.0 \mathrm{~mm}^{3}$; (c) and (d) $1.6 \times 1.6 \times 6.0 \mathrm{~mm}^{3}$. (a) and (c) were taken from the cardiac diastolic phase; (b) and (d) were taken from the systolic phase. The other imaging parameters: spoiled radial FLASH, FOV 256×256 mm², FA $8^{\circ}, B W 1560 \mathrm{~Hz} / \mathrm{Pixel}$. 


\subsubsection{Temporal Resolution}

Fig 6.7 shows an example that increases the temporal resolution by fewer view numbers, while keeping the image matrix size constant to maintain the spatial resolution. The frames presented here were chosen from six independent real-time cardiac image series with free breathing and without ECG synchronization, all from systolic phase. A FOV of $256 \times 256 \mathrm{~mm}^{2}$, a matrix size of $128 \times 128$, and a slice thickness of $8 \mathrm{~mm}$, were remained constant throughout the whole measurements. This resulted in the same spatial resolution of $2.0 \times 2.0 \times 8.0 \mathrm{~mm}^{3}$. Whereas the number of views was reduced from 205 (full sampling) to 125, 85, 65, 45, and even to an extreme case of only 35 with 6-fold undersampling, which resulted in an increasing effective temporal resolution from $12 \mathrm{fps}$ to $70 \mathrm{fps}$ correspondingly.

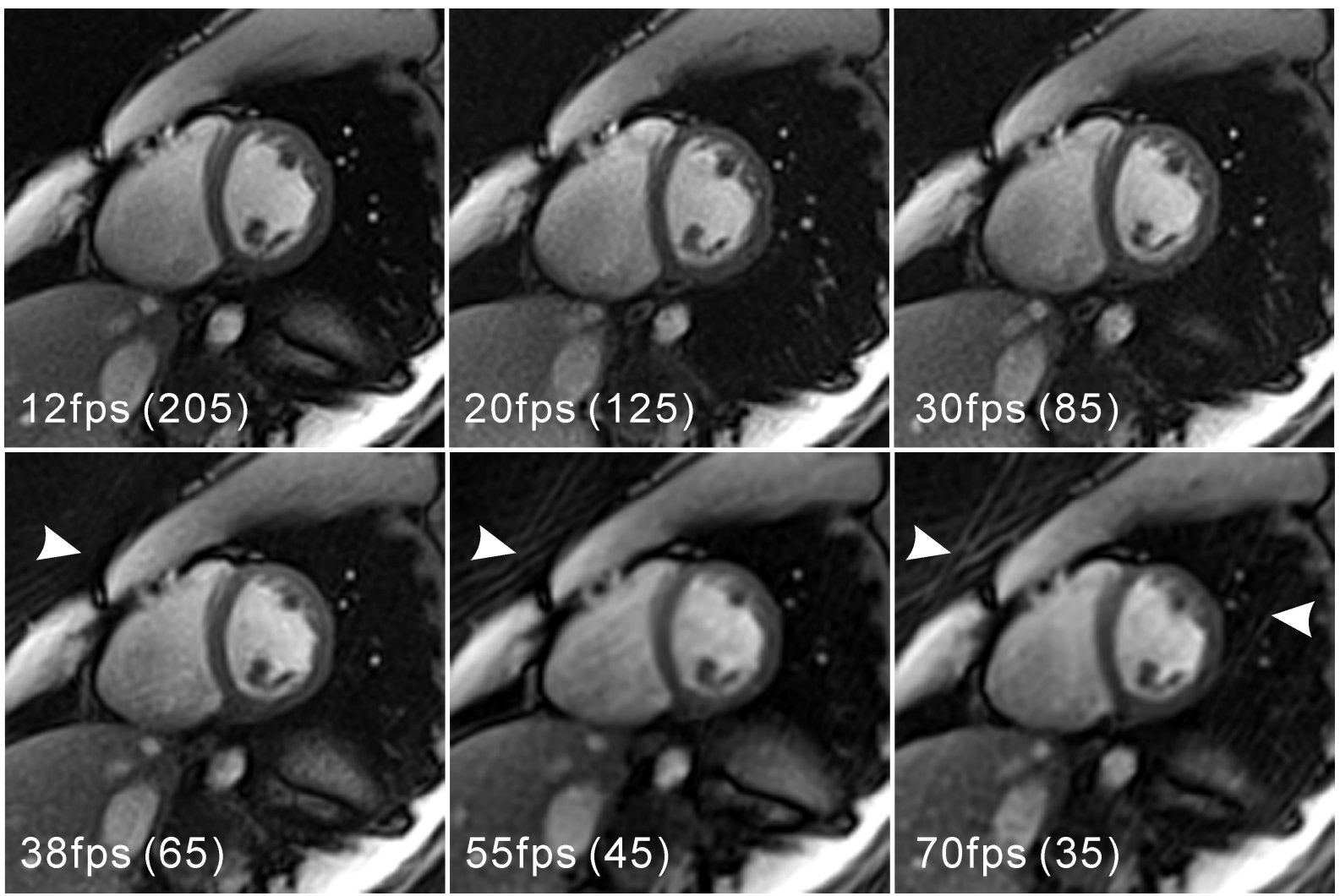

Figure 6.7 Radial FLASH RT-MRI of the human heart with different temporal resolution and 2.0×2.0×8.0 $\mathrm{mm}^{3}$ spatial resolution (short-axis view). Reducing the number of views resulted in blurring, decreased SNR and gradually stronger streaking artifacts (arrow heads), while maintaining most of the object information and the contrast. Imaging parameters: spoiled radial FLASH, FOV $256 \times 256 \mathrm{~mm}^{2}$, matrix size $128 \times 128, T R / T E 2.02 / 1.3 \mathrm{~ms}, F A 8^{\circ}, B W 1560 \mathrm{~Hz} / \mathrm{Pixel}$.

In the full sampling case, the update time (TU) was $83 \mathrm{~ms}$, which is comparable to the normal cardiac phase (e.g. the QRS-complex and the end-systolic phase is normally about 60 to 100 ms, while the end-diastolic phase is slightly longer, from which the presented image in Fig 6.7 
was chosen), and is not efficient to update the whole rapid motion process. At the same time, the image acquisition time (TA) of $414 \mathrm{~ms}$ in this case is also too long compared to the object movement, which often causes smearing of structures (i.e. myocardium). With increased temporal resolution, especially in the extreme case with highly undersampling, it resulted in blurring, decreased SNR and gradually stronger streaking artifacts (arrow heads). However, most of the object information and the contrast between blood and myocardium still remain visible. Furthermore, the rapid dynamic process can be better delineated with shortened $T A$ and $T U$. This is better demonstrated in Fig 6.8, where two temporal resolutions - 20 fps and 38 fps - are compared in an anatomically defined 4-chamber view.

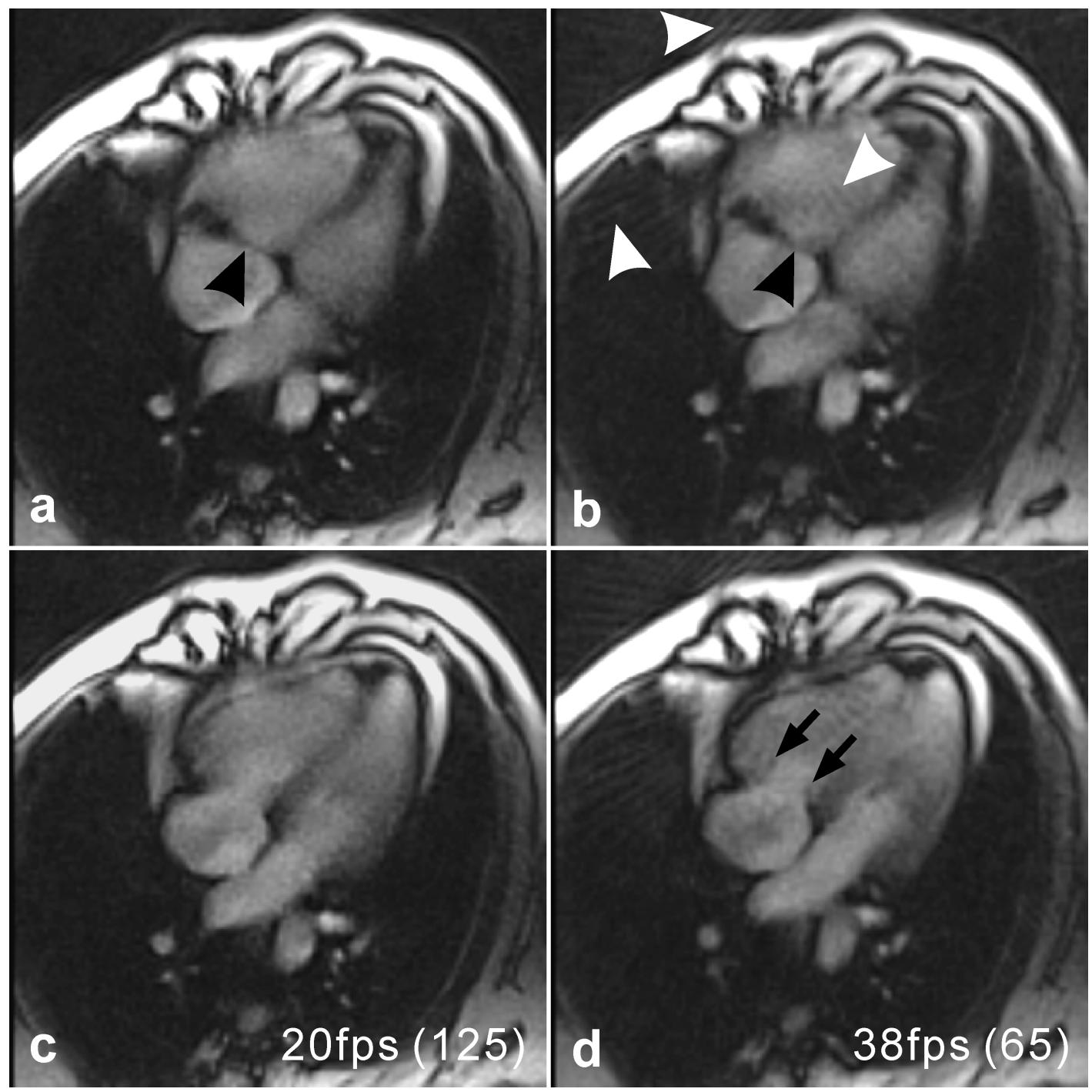

Figure 6.8 Radial FLASH RT-MRI of the human heart with different temporal resolution and $2.0 \times 2.0 \times 8.0$ $\mathrm{mm}^{3}$ spatial resolution (4-chamber view). (a) and (c) at $20 \mathrm{fps}$; (b) and (d) at $38 \mathrm{fps}$. (a) and (b) were taken from the end-systolic phase; (c) and (d) were taken from the diastolic phase. The other imaging parameters were the same as that in Fig 6.7. Black arrow heads in (a) and (b) indicate the closure of the tricuspid valve at the end-systolic phase; small black arrows in (d) indicate the visibility of the tricuspid valve at the enddiastolic phase at a temporal resolution of $38 \mathrm{~Hz}$; white arrow heads indicate streaking artifacts due to undersampling that do not interfere the monitoring of the cardiac motion. 
In Fig 6.8, a and c were acquired at 20 fps, with 125 views and a TA about $250 \mathrm{~ms}$; b and d were acquired at $38 \mathrm{fps}$, with 65 views and a TA about $130 \mathrm{~ms}$. At end-systolic phase (a and b) when the ventricles contract and the atrioventricular valves close, the closure of the tricuspid valve (right atrioventricular valve) can be seen with both temporal resolutions, as indicated by the black arrow heads. However, during the diastole phase when the atria and ventricles are relaxed, the atrioventricular valves are open, allowing blood to flow to the ventricles. This is hardly observable at $20 \mathrm{fps}$ with $T U 50 \mathrm{~ms}$, but at $38 \mathrm{fps}$ (even higher than the normal video data stream at about $24 \mathrm{fps}$ ) with $T U 26 \mathrm{~ms}$, the tiny valves with very fast motions can be captured, as indicated by the black arrows. Streaking artifacts due to both undersampling and motions are also visible in this case (white arrow heads), but do not interfere with the monitoring of the cardiac motion.

This proposed RT-MRI method applies also to the situation at higher spatial resolution with relatively slower motion speed. In Fig 6.9, both a and b were acquired during the mouth opening of a volunteer to study the displacement of temporomandibular joint (TMJ). The movement speed was controlled by presenting a visual cue and was almost identical for both examinations (about $20 \mathrm{~s}$ for the opening period). Images in Fig 6.9a were acquired at $3 \mathrm{fps}$ (385 views and 5 interleaves) using a $192 \times 192 \mathrm{~mm}^{2}$ FOV and a $256 \times 256$ matrix size, resulting in an in-plane spatial resolution of $0.75 \times 0.75 \mathrm{~mm}^{2}$. To increased the temporal resolution, Fig 6.9b were acquired with 235 views and 5 interleavesat at $5 \mathrm{fps}$. At the same time, a slightly smaller FOV of $120 \times 120 \mathrm{~mm}^{2}$ and a matrix size of $160 \times 160$ were used to avoid strong undersampling, and to yield the same in-plane resolution. The presented frames in Fig 6.9a were eight neighboring images without time interval, while the eight frames in Fig $6.9 \mathrm{~b}$ were chosen from 14 images with roughly one time frame interval for correspondingly the same motion period as that in Fig 6.9a. All images shown here were zoomed into the same area of interest for demonstration. In this subject, at higher temporal resolution of $5 \mathrm{fps}$, the smearing and jumping of the mandibular condyle - possibly due to a potential pathological reason - is better resolved.

All these results demonstrate the high capability of the developed radial FLASH RT-MRI method in achieving rapid signal acquisition, efficient encoding, fast reconstruction and smooth image update. More specific results from individual applications are presented and discussed in the next chapter. 

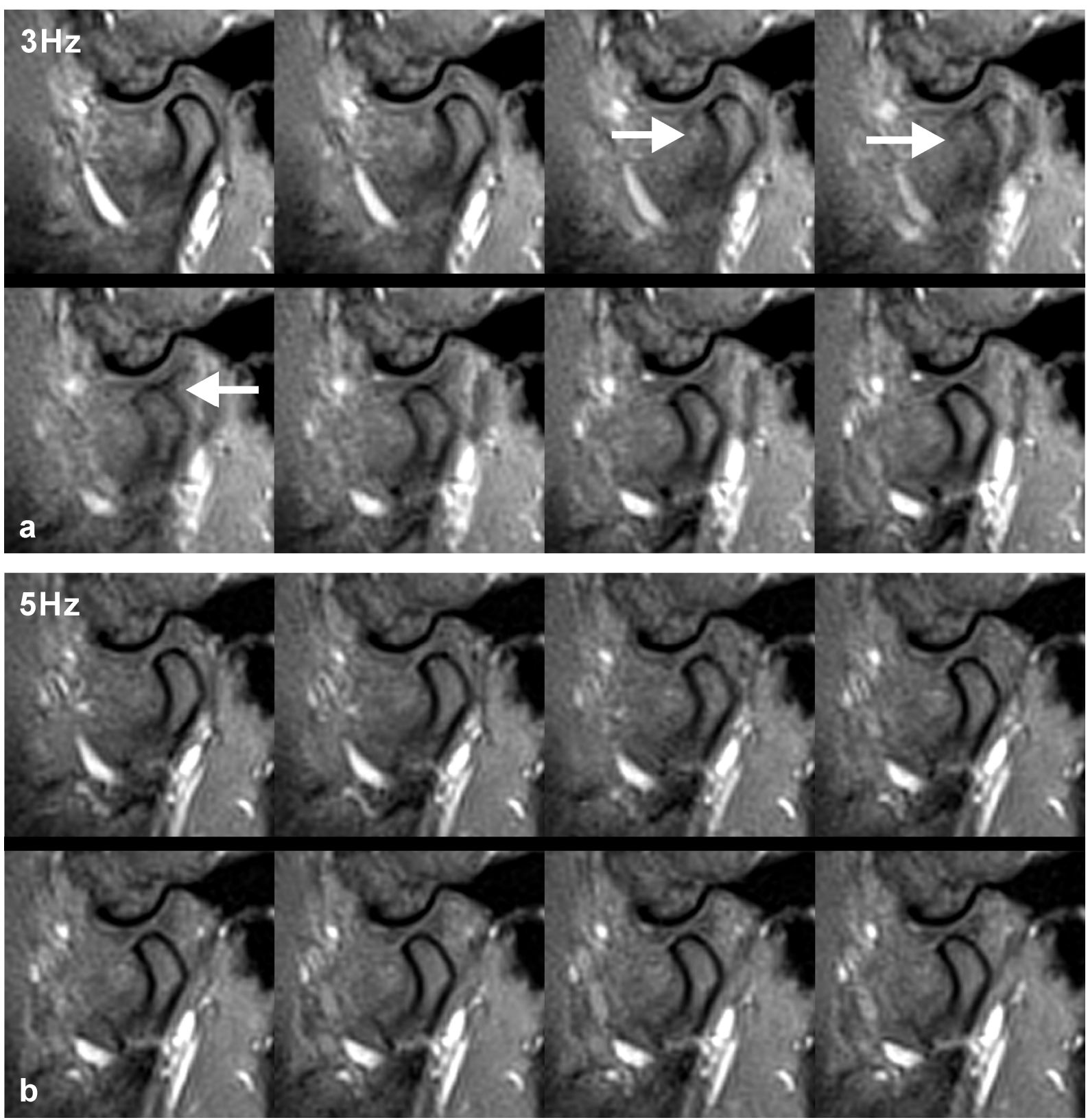

Figure 6.9 Radial FLASH RT-MRI of the TMJ movement with different temporal resolution and $0.75 \times 0.75 \times 5 \mathrm{~mm}^{3}$ spatial resolution. (a) at $3 \mathrm{fps}$; (b) at $5 \mathrm{fps}$. The smearing of the condyle (arrows) due to data inconsistencies and sharing during abrupt motion in (a) was markedly improved in (b). The other imaging parameters: refocused radial FLASH, FOV $192 \times 192 \mathrm{~mm}^{2}$, matrix size $256 \times 256$, TR/TE 4.33/2.20 $\mathrm{ms}, F A 20^{\circ}, B W 810 \mathrm{~Hz} / \mathrm{Pixel}$.

\subsection{Receiver Channel Compression}

The increasing use of receiver coils with very high numbers of independent elements considerably increases the computational load for image reconstruction. In fact, when taking advantage of 32-element receive coils for the MRI system used here, it will cause a noticeable delay between the end of signal acquisition and display of the last image. Therefore, this 
section describes a software-based receive channel compression method [265, 266] that has been implemented to solve this problem. Accordingly, incoming signals from the individual coil elements are combined into a small set of compressed channels. Because this combination is much faster than a full processing of the signals from all receive channels, the images are reconstructed and displayed in real time - with negligible latency as evident from the synchrony with the acoustic gradient sounds.
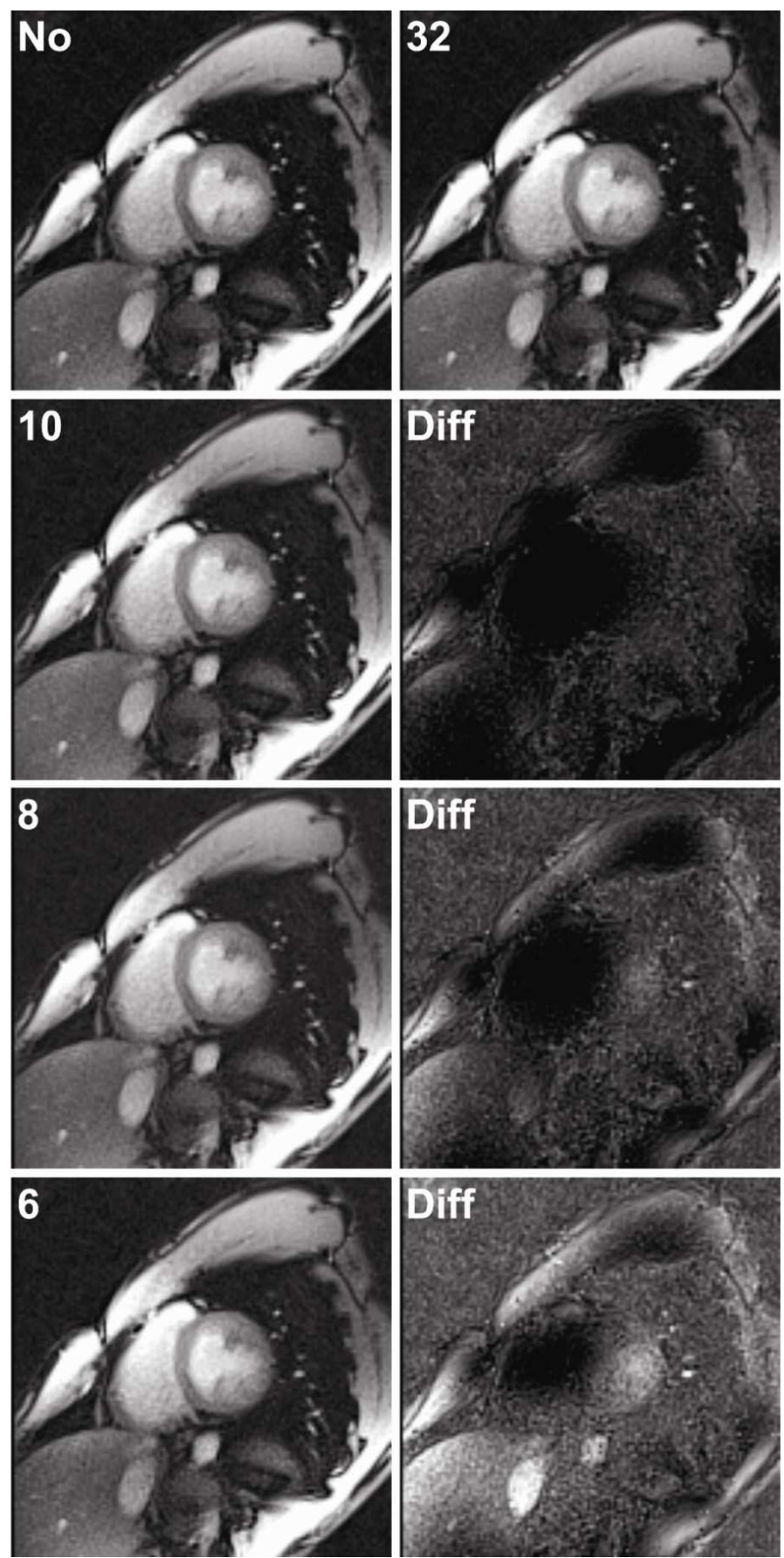

Figure 6.10 Receiver channel compression for spoiled radial FLASH RT-MRI of the heart (short-axis view). The images refer to reconstructions with no channel compression or the use of $32,10,8$, and 6 principal components and the corresponding difference images (Diff) to the 32-channel reconstruction (individual windowing to emphasize the small signals). Imaging parameters were the same as in Fig 6.7. 
Briefly, channel compression is achieved by generating linear combinations of the incoming signals, where the complex-valued combination weights vary for every input and output channel. These weights are estimated using a principal component analysis (PCA) using data acquired during a few preparation scans (typically employed for steady-state equilibration) and kept fixed afterwards. For the PCA, a covariance matrix is constructed by calculating the covariances of the received signals among all combinations of channels. In the case of the 32element receiver coil, this yields a $32 \times 32$ matrix that describes the inter-channel correlations. By conducting a singular value decomposition (SVD) of the covariance matrix, a transformation matrix is obtained that contains the desired weights for combining the receive channels into uncorrelated eigenmodes. The weights are directly sorted in descending order according to the energy content of the corresponding eigenmodes. Because most coil arrays carry a certain degree of redundancy due to spatial overlap of the sensitivities from individual coil elements, the higher eigenmodes contain only marginal image information. Therefore, a compression without a recognizable loss in image quality may be achieved by restricting the combination to only a limited number of channels. The performance of the implemented channel compression technique is demonstrated in Fig 6.10.

In general, the degree of usable compression depends on the object under investigation and, more importantly, on the geometry of the coil array. The channel compression method was therefore integrated into the system's software framework and the feature provided as a user-selectable tool for radial FLASH real-time MRI sequences. 


\section{Chapter 7}

\section{Biomedical Applications of Real-time MRI}

Ever since the conception of RT-MRI [19, 203, 204] the technical development has been driven by specific applications [202, 205-207, 215]. One basic use is to improve the efficiency of MRI examinations, in which the reconstruction and display can proceed without delay to the signal acquisition and continuous sampling of the imaging object. Moreover, as demonstrated during the experimental implementation on a 3T MRI system in the previous chapter, the proposed method based on the FLASH physics, radial $k$-space encoding, and sliding window with view sharing reconstruction, supports and opens up a wider range of applications to various dynamic processes.

In this chapter, a few selected applications of potential clinical interest are presented, namely the functional assessment of the ocular movement, the temporomandibular joint, the musculoskeletal system, speech production, swallowing process and the cardiovascular system. Other more preliminary trials ranging from tongue movement during chewing to abdominal imaging during free breathing have also been carried out, but are not shown here. Each subchapter begins with background information about the currently applied MRI techniques. Afterwards, RT-MRI results are presented to demonstrate the strength of the proposed method and its significance to address specific issues of these applications. Furthermore, comparisons with the available literature are offered, and the potential uses in each application are also discussed.

\subsection{Ocular Movement}

Over a lifetime, movements of the eyes performed by an average human can be literally billions of times - even during sleep. Muscles surrounding the eye are among the most active in the body, making possible some one hundred thousand movements a day. If any one of the muscles is not sufficiently precise, e.g. under pathological conditions, and cannot function coordinately, numerous symptoms may occur affecting the eye movement as well as the 
vision. The facial expression may also be distorted since the harmony between the muscles is gone as in the case with squinting (strabismus).

Surrounding each eyeball are six muscles (so-called extraocular muscles): one pair each for horizontal, vertical, and side-to-side oblique movements (Fig 7.1). For example, the primary function of the medial rectus muscle (MRM) is to move the eye towards nose, while the lateral rectus muscle (LRM) is to move the eye away from nose. Superior rectus muscle (SRM) and inferior rectus muscle (IRM) mainly serves to raise and lower the eye, respectively. Superior oblique muscle (SOM) and inferior oblique muscle (IOM) are to rotate the eye. However, each member of the pair, as well as all three groups, must work together in coordination to control the eye movement, so that both eyes turn to the object of interest and its image falls on both retinas.
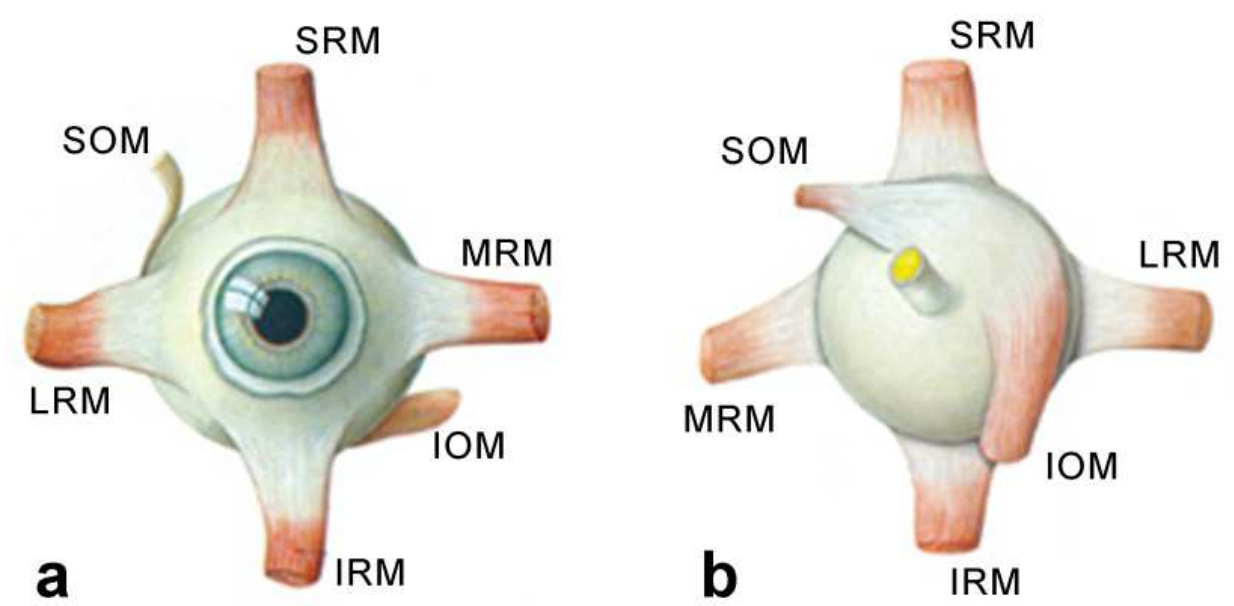

Figure 7.1 Anatomy of the eye (right) muscles in a (a) front view and a (b) back view, respectively.

MRM: medial rectus muscle SRM: superior rectus muscle SOM: superior oblique muscle
LRM: lateral rectus muscle IRM: inferior rectus muscle IOM: inferior oblique muscle

For ocular imaging, many modalities have been used. Due to its better contrast in the soft tissues than computerized tomography (CT), and its higher precision in qualitative studies over ultrasound [267], MRI is always preferable for imaging extraocular muscles in clinic. It has been used for observing changes in muscle shapes such as atrophy and hypertrophy and for monitoring eye movement and muscle contraction in the orbit [268, 269].

However, imaging of the ocular movement has so far been applied almost only with pseudo-dynamic MRI, so-called cine mode MRI. In this method, a strip of paper or card with separate, normally equidistant, marks (either in transversal or in sagittal plane) was suspended in front of patients. Each mark provided a point of fixation for the eyes, while a still image 
frame was acquired. The patient was requested not to blink during imaging. Finally, all images were subsequently played as a cine loop. Although different acquisition techniques have been employed, such as spoiled FLASH [270, 271], refocused FLASH [272-274], T1WSE [275, 276] or TSE [277], the in-plane spatial resolution was limited around $1.2 \times 1.2 \mathrm{~mm}^{2}$ to $1.5 \times 1.5 \mathrm{~mm}^{2}$, and the acquisition time of one image (TA) was about $15-30 \mathrm{~s}$, which means that the patients must fix their eyes for such a period of time. Thus, the total imaging time for one pseudo-dynamic cycle was about 3-4 min. Although the latest work with such kind of approach employed turbo-FLASH sequence for signal acquisition which shortened TA to 1-5 s due to fewer RF excitations [278, 279] as described in subchapter 3.2, it was still unfeasible for true dynamic imaging and real-time monitoring. The only one under the true dynamic condition that is available in the publications employed Cartesian FLASH to investigate nystagmus [280]. It reached a temporal resolution of $4.4 \mathrm{fps}$, but at the expense of lower spatial resolution of $2.5 \times 2.0 \mathrm{~mm}^{2}$, and also larger slice thickness of $5.0 \mathrm{~mm}$ to regain $\mathrm{SNR}$, which was not suitable for imaging the tiny structures in obit.

As a short summary, the studies of the ocular movement with the currently available MRI techniques suffer from the following disadvantages: First, overall image quality depends on how well the patient can control head movement, concentrate on the fixation point, and avoid blinking. In a few cases [275], local anesthetic eye drops were even required to inhibit the blink reflex. Second, long imaging times and preferred more fixation points for smoother video loop or the eye movement may lead to patient fatigue. Third, each cine video is simply a series of static images viewed sequentially and rapidly; therefore, appreciation of true physiological eye movements and voluntary pursuit eye movements is not possible.

All these difficulties are bypassed when applying the proposed radial FLASH real-time MRI. Fig 7.2 shows an example of studying ocular movement (healthy volunteer, female, 30 years old) in three different imaging planes. Because of the small anatomical structures that need to be imaged, a high spatial resolution of $0.75 \times 0.75 \mathrm{~mm}^{2}$ with a relative thin slice of 4 mm was used for a FOV of $192 \times 192 \mathrm{~mm}^{2}$ and an image matrix size of $256 \times 256$. This led to a relatively low temporal resolution of 3 fps with 375 views and 5 interleaves. During the dynamic measurement, the volunteer was asked to rotate the eye at a slow but continuous speed, resulting in about 7-10 s for a movement from ultimate left to ultimate right. Fig 7.2a and $\mathrm{b}$ were acquired in the transversal and oblique sagittal plane respectively, passing along the central axis of the optic nerve $(\mathrm{ON})$, when the volunteer rotated the eyes, from left endpoint to right end-point position (i.e. transversal eye movement) and from up end-position to down end-position (i.e. vertical eye movement), respectively. Fig 7.2c was acquired in the 
coronal plane behind the globe when the volunteer was asked to do a $360^{\circ}$ rotation from extreme medial gaze to extreme lateral gaze (i.e. clockwise movement). A 32-channel head coil was used for all the examinations, so that both eyes could be studied at the same time. All images were zoomed to the left eye.

In all studies presented here, first of all, the motions of the globe, optic nerve and muscles in the corresponding movement, i.e. MRM, LRM, SOM (not presented here) in transversal movement, SRM and IRM in vertical movement, MRM, LRM, SRM and IRM in $360^{\circ}$ rotation, were clearly delineated throughout the whole process. Second, the dark area obscuring the lens in Fig 7.2a (white arrow head) was caused by blinking of the eye. It is well demonstrated here that with the present technique, the tissues of interest, i.e. ON, MRM and LRM in this case, was not affected by such unavoidable motion, which is not achievable by the conventional static or pseudo-dynamic MRI. Third, lid movement (white arrows) with different vertical positions of the globe was seen in the oblique sagittal plane in Fig 7.2b during vertical movement of the eye. With proper slice positioning, the tarsus and the lid retractors could also be assessed (not presented here). Fourth, the contractility of individual muscles was also seen, for example, the thickening of the SRM (black arrows) as the result of muscle relaxation and the thinning of the IRM (black arrow head) as the result of contraction when the eye moved to the extreme downward position in Fig 7.2c.

Although more experiments are required, for example to assess the evalutation of saccadic eye movements, the results already demonstrate the feasibility of the radial FLASH RT-MRI to observe and pursuit voluntary eye movements. By monitoring the anatomical changes, different dynamic disorders can be investigated, for example, the impaired function of muscles as seen in many diseases such as strabismus [275, 276] or posttraumatic detachment of eye muscles. When taking the cornea and the lens as landmarks, the movement of the globe, and the length as well as the thickness changes of the antagonistic and agonistic muscles during true physiological movement can be quantified. In addition, it may also be useful for eye surgery planning (e.g. the decompression of the orbit by enlarging the orbit into the paranasal sinus) [271], as well as selection and implementation of therapy for eye movement disorder. 

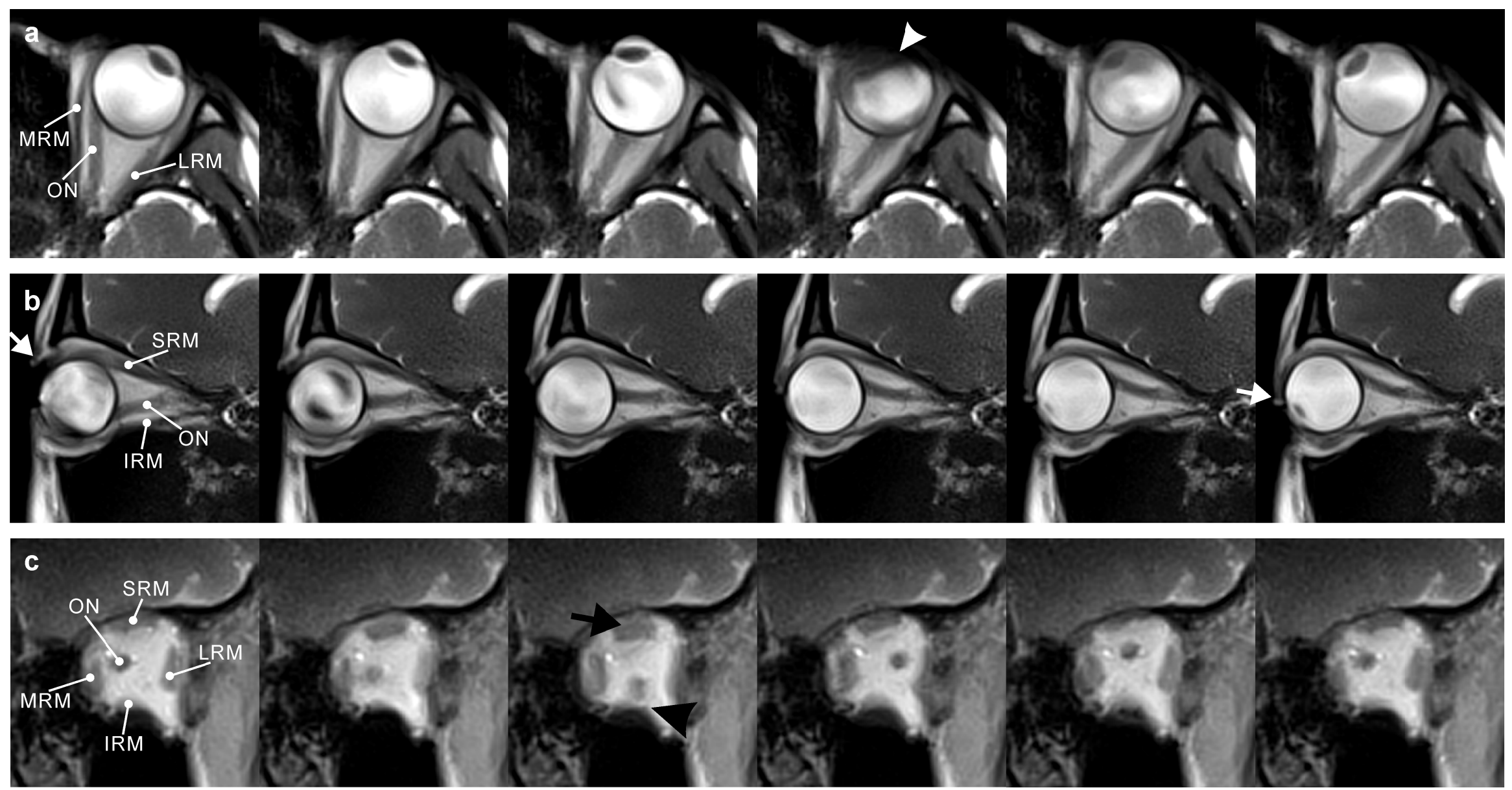

Figure 7.2 Radial FLASH RT-MRI of ocular movement (magnified to the left eye). (a) Left-and-right transversal movement with transversal image plane. (b) Up-and-down vertical movement with oblique sagittal image plane. (c) Media-and-lateral clockwise movement with coronal image plane. White arrow in (a) indicates the moment when the volunteer was blinking; white arrow heads in (b) indicate the lid movement; black arrow and black arrow head in (c) indicate the thickening and thinning of the SRM and IRM respectively.

MRM: medial rectus muscle SRM: superior rectus muscle
LRM: lateral rectus muscle IRM: inferior rectus muscle
ON: optical nerve 


\subsection{Temporomandibular Joint}

The temporomandibular joint (TMJ) is the joint of the jaw located in front of the ear. It is a hinge and gliding joint and is the most constantly used joint in the body. At the same time, it is also considered the most complex joint in the human body because the related bones, joints, and muscles provide for an infinite range of combined movements and functions (e.g. rotational movements, sliding movements), unlike any other joint in the body. Fig 7.3a shows the normal TMJ anatomy with enlarged region of interest. The round upper end of the lower jaw, or the movable portion of the joint, is called mandibular condyle (5). It is elliptically shaped with its long axis oriented mediolaterally. The socket is called articular fossa (3). Between the condyle and the fossa is the articular disc (2), also called meniscus, made of cartilage. It is a fibrous, saddle shaped structure that acts as a cushion to absorb stress and allows the condyle to move easily when the mouth opens and closes during chewing, speaking and swallowing. The disc varies in thickness: the thinner, central intermediate zone separates thicker portions called the anterior band and the posterior band. Anteriorly, the disc is connected with the lateral pterygoid muscle (4) which acts to lower the mandible, open the jaw, and help in moving the jaw from side to side (mastication). Posteriorly, the disc is contiguous with attached posterior ligament (1) and tissues called the bilaminar zone which plays an important role in allowing the condyle to move forward.
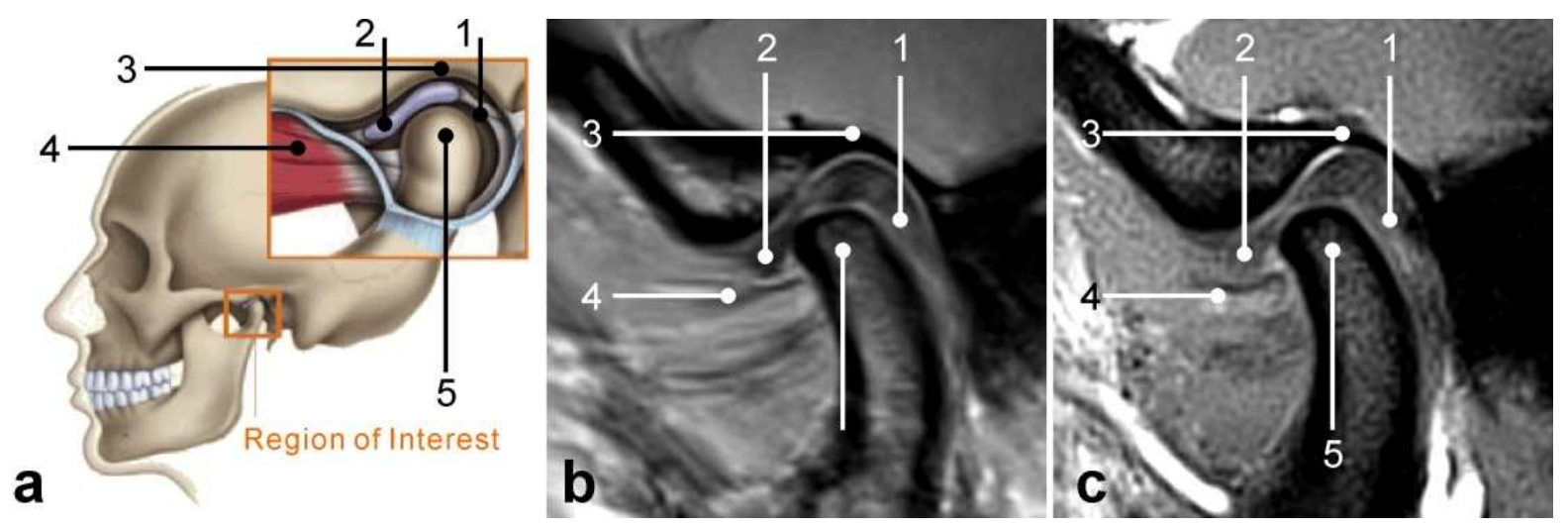

Figure 7.3 Anatomy of the temporomandibular joint. (a) Schematic illustration with region of interest. (b) Static image acquired with PDW-TSE. (c) real-time image acquired with refocused radial FLASH. In both (b) and (c), a very high in-plane resolution of $0.4 \times 0.4 \mathrm{~mm}^{2}$ with a slice thickness of $2.5 \mathrm{~mm}$ was used.
1. posterior ligament
2. articular disc
4. muscle
5. mandibular condyle
3. articular fossa 
Normally, the disc and the condyle fit in the socket when the jaw is closed. As the jaw opens, the condyle moves forward and the disc slides forward correspondingly. Pathology of TMJ is often called temporomandibular joint disorder (TMD) or TMJ syndrome, which refers to a group of acute or chronic problems involving the joint themselves and the tissues associated with them. The disorder and resultant dysfunction can result in significant pain and impairment, such as a jaw that is locked in position or difficult to open, biting problems, and popping sounds when biting.

Because of the advantages that were mentioned before, including the non-invasiveness comparing to arthrography, arthroscopy and CT in medical examination of joints, MRI is frequently used to study the disc position and morphology. Fig 7.3b shows a typical PDweighted (PDW) TSE image of the TMJ, at a slightly opened mouth position. As the standard imaging protocol that is often used in clinic, however, it took approximately $20 \mathrm{~s}$ to acquire a whole image. From the late 80s, with the invention of the FLASH technique, various imaging studies have been carried out in cine mode MRI under the pseudo-dynamic condition [281295]. In such studies, a bite block or device was used to open the mouth step by step or the subject was asked to simply open the mouth incrementally, and to hold for a typical time of 20 to $30 \mathrm{~s}$ for each position for imaging. Thus a whole open- and close cycle took about 4 to 6 minutes and the acquired images were retrospectively combined. As discussed for imaging of the ocular movement, such passive movement is not physiologically equivalent to the active movement, besides its long measurement time. It is, therefore, preferable to apply true dynamic imaging.

Numerous tests of the proposed RT-MRI variants demonstrated that refocused FLASH with T1/T2 contrast is superior to the spoiled version in terms of SNR and tissue contrast, for better definition of disc shape and location. This is well demonstrated in Fig 7.3c, with very high spatial resolution of $0.4 \times 0.4 \mathrm{~mm}^{2}\left(128 \times 128 \mathrm{~mm}^{2}\right.$ FOV and $320 \times 320$ image matrix $)$ and $2.5 \mathrm{~mm}$ slice thickness, which served as a localizer before the dynamic study. In all studies, a bilateral 4 array coil (CPC coil with two independently and freely movable 4-element coils, NORAS MRI Products, Höchberg, Germany) was used as shown in Fig 1.4. Noticeably, compared to the image quality from static or pseudo-dynamic image as shown in Fig 7.3b at the same mouth opening position, all tissues of interest are clearly presented, despite an overall SNR descrease due to the fast acquisition.

Fig 7.4 shows an example of normal TMJ displacement during mouth opening and closing by employing the proposed radial FLASH RT-MRI from a healthy volunteer (female, 30 years old). Fig 7.4a demonstrates the positioning of the imaging slice. It was oriented in an 

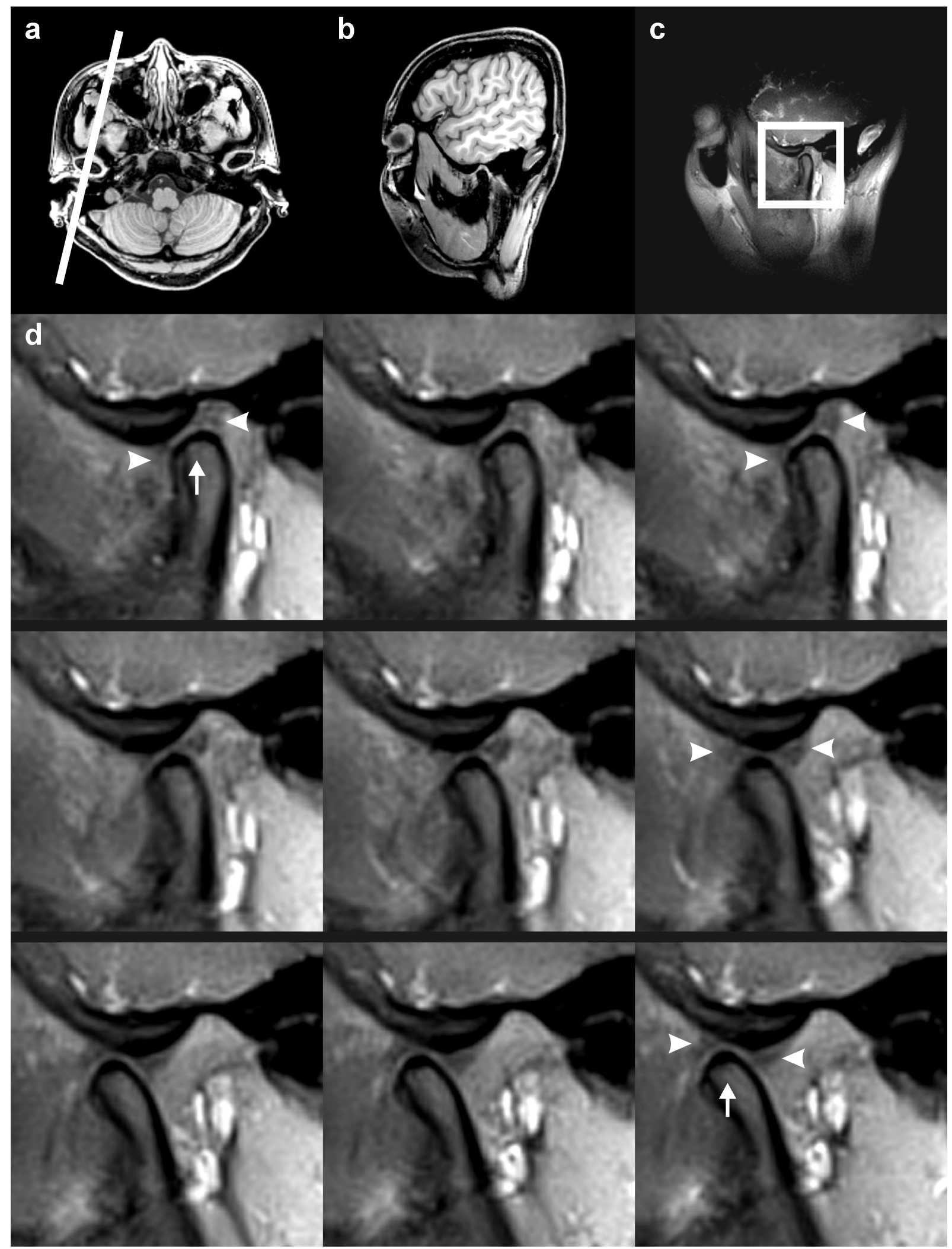

Figure 7.4 Radial FLASH RT-MRI of normal TMJ movement at $0.75 \times 0.75 \times 5 \mathrm{~mm}^{3}$ spatial resolution and 3 fps temporal resolution. (a) and (b) Slice positioning. (c) Obtainable FOV. (d) Normal TMJ movement during the opening of the mouth. Arrows indicate the anterior displacement of the mandibular condyle, and arrow heads indicate the corresponding movement of the articular disc. The imaging parameters: refocused radial FLASH, FOV 192×192 $\mathrm{mm}^{2}$, matrix size 256×256, TR/TE 4.33/2.20 ms, FA 20,$B W 810 \mathrm{~Hz} / \mathrm{Pixel}$. 
oblique sagittal plane and perpendicular to the horizontal long axis of the mandibular condyle. The resultant slice was shown in Fig $7.4 \mathrm{~b}$, and the obtainable FOV as well as the general image quality was shown in Fig 7.4c. Imaging parameters were $T R 4.33 \mathrm{~ms}, T E 2.10 \mathrm{~ms}, B W$ of $810 \mathrm{~Hz} / \mathrm{Pixel}$, and $F A$ of $20^{\circ}$ for RF excitation. The images covered a $192 \times 192 \mathrm{~mm}^{2} \mathrm{FOV}$ with a matrix size of $256 \times 256$, which resulted in an in-plane spatial resolution of $0.75 \times 0.75$ $\mathrm{mm}^{2}$. The slice thickness was $5 \mathrm{~mm}$ and 385 views were used corresponding to a fully sampled dataset. The views were arranged in an interleaved 5 segments radial scheme, so that image updates were reconstructed every $385 / 5=75$ spokes corresponding to a frame rate of 3 fps. Images in $d$ are magnified to the region of interest, indicated by the white rectangle in Fig 7.4c. They were selected about every $3 \mathrm{~s}$ from the image series from the mouth close position to the full open position. The original study was about $55 \mathrm{~s}$ duration and covered a full cycle of slowly and voluntary mouth opening and closing.

Apart from showing the anterior displacement of the condyle as well as the corresponding forward movement of the disc relative to the condyle and the fossa during the entire movement, two phases or two distinct motions of the dynamic process were well visualized. The three images in the first row of the Fig 7.4d represent the first phase when the condyle (white arrow) rotates around a horizontal axis through the condylar head, while its position and that of the disc (white arrow heads) hardly change. The rest of the images represent the second phase when the mouth is wider opened, the condyle and the disc move together anteriorly. When the mouth is fully open, the condyle lies beneath the disc.

From the late 90s, a few dynamic studies based on Cartesian encoding were applied with different pulse sequences [296-302]. When compared to the experimental findings from these previous studies, the present results show superior image quality, with respect to the technical imaging parameters like spatial and temporal resolution, and to the visualization of the TMJ structures during movement.

Fig 7.5 presents the study results from a volunteer (male, 23 years old) with TMJ disorder. The imaging parameters were the same as in Fig 7.4d. Fig 7.5a shows the TMJ movement on the asymptomatic left side, while Fig 7.5b shows the TMJ movement on the right side with mouth opening restriction and grinding noises. Noticeably in $b$, as the disc translates anteriorly, the whole disc remains in front of the condyle (black arrows) and the ligament becomes abnormally stretched and attenuated. At full mouth opening, the disc remains anteriorly displaced, which is termed anterior displacement without reduction. In the first image of Fig 7.5a at the mouth close stage, the disc already shows an anterior displacement (white arrow head) as frequently described in textbooks [303], when compared to the disc 

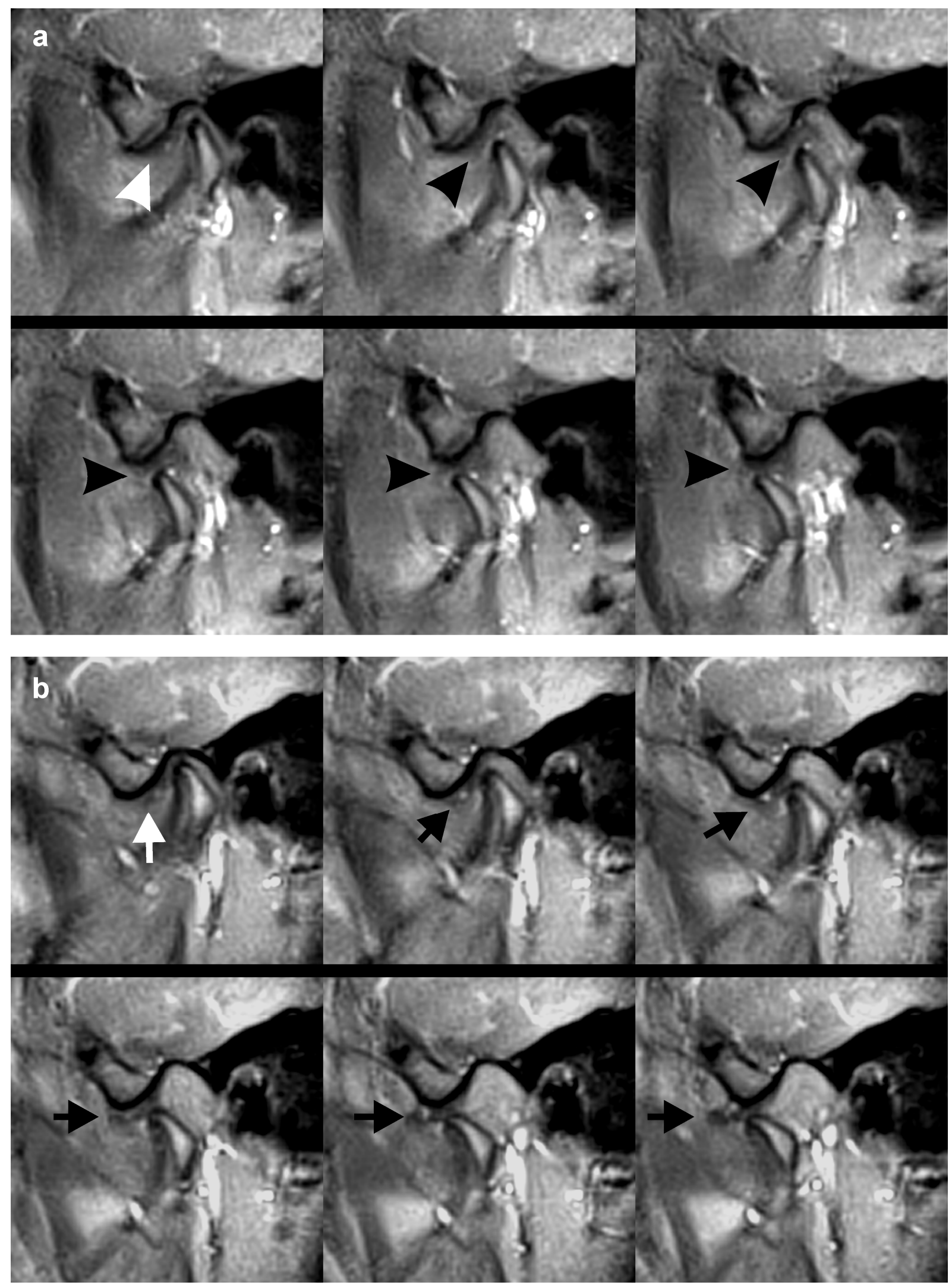

Figure 7.5 Radial FLASH RT-MRI of TMJ with internal derangement. (a) and (b) are two studies from the asymptomatic left side and the symptomatic right side, respectively. White arrow head in (a) and white arrow in (b) indicate the close mouth position of the disc from left and right side, respectively; black arrow heads in (a) and black arrows in (b) indicate the displacement of the disc from left and right side, respectively, during TMJ movement. The imaging parameters were the same as that in Fig 7.4. 
location relative to the condyle in the first image of Fig $7.4 \mathrm{~d}$ and the symptomatic right side in Fig 7.5b (white arrows). However, starting from the mouth opening, the disc was captured and remained in the right position relative to the condyle and the fossa (black arrow heads), therefore, no clinical symptoms had shown on this side. In short, for the other cases of anterior displacement with reduction in the internal derangement of the TMJ, the displaced disc will return to its normal position when the condyle reaches a certain point, and when the reduction of the disc happens, the patient often feels a pop or click in the joint.

As presented above, radial FLASH (refocused) RT-MRI demonstrates a high capability to provide the clinician with additional information in the evaluation of functional movement disorders, potential pre- or post-surgery planning, and diagnosis of joint pathology.

\subsection{Musculoskeletal System}

Muscles, bones, joints and tendons work in concert to create precise movements. As having been demonstrated in the TMJ case, a functional evaluation of the musculoskeletal system may arise as another application of radial FLASH RT-MRI. Typically, for a given joint, interactions of the soft tissues and bony anatomical features that comprise this joint are studied through a specific range of motion, e.g. bending of the knee. So far a variety of pulse sequences and joint positioning strategies have been used in joint MRI, either during passive movement [304-311] as in pseudo-dynamic studies or active movement [183, 312-318] as in dynamic studies, both of which are also termed joint "kinematic" MRI [319, 320]. Since the value of the motion imaging lies in the need to precisely identify the position and morphological changes of particular anatomical structures with physiological relevance, only the active movement is addressed in the present real-time functional joint MRI.

Because a joint normally involves tissues with relatively small size and complex anatomy, and on the other hand, its movement is voluntary with controlled speed, the imaging parameters and protocols employed in this application aim at a high spatial resolution with relatively low temporal resolution, similar to the TMJ application. Furthermore, in-phase and opposed-phase imaging based on the chemical shift mechanism offer different contrasts for specific tissues, as already shown in Fig 7.3. So far, the developed RT-MRI technique has been applied to assess various joints in the human body including the ankle, cervical spine, knee, shoulder, wrist, and TMJ as already discussed in the previous section.

Fig 7.6 presents examples from three real-time dynamic measurements. Flexion of the 

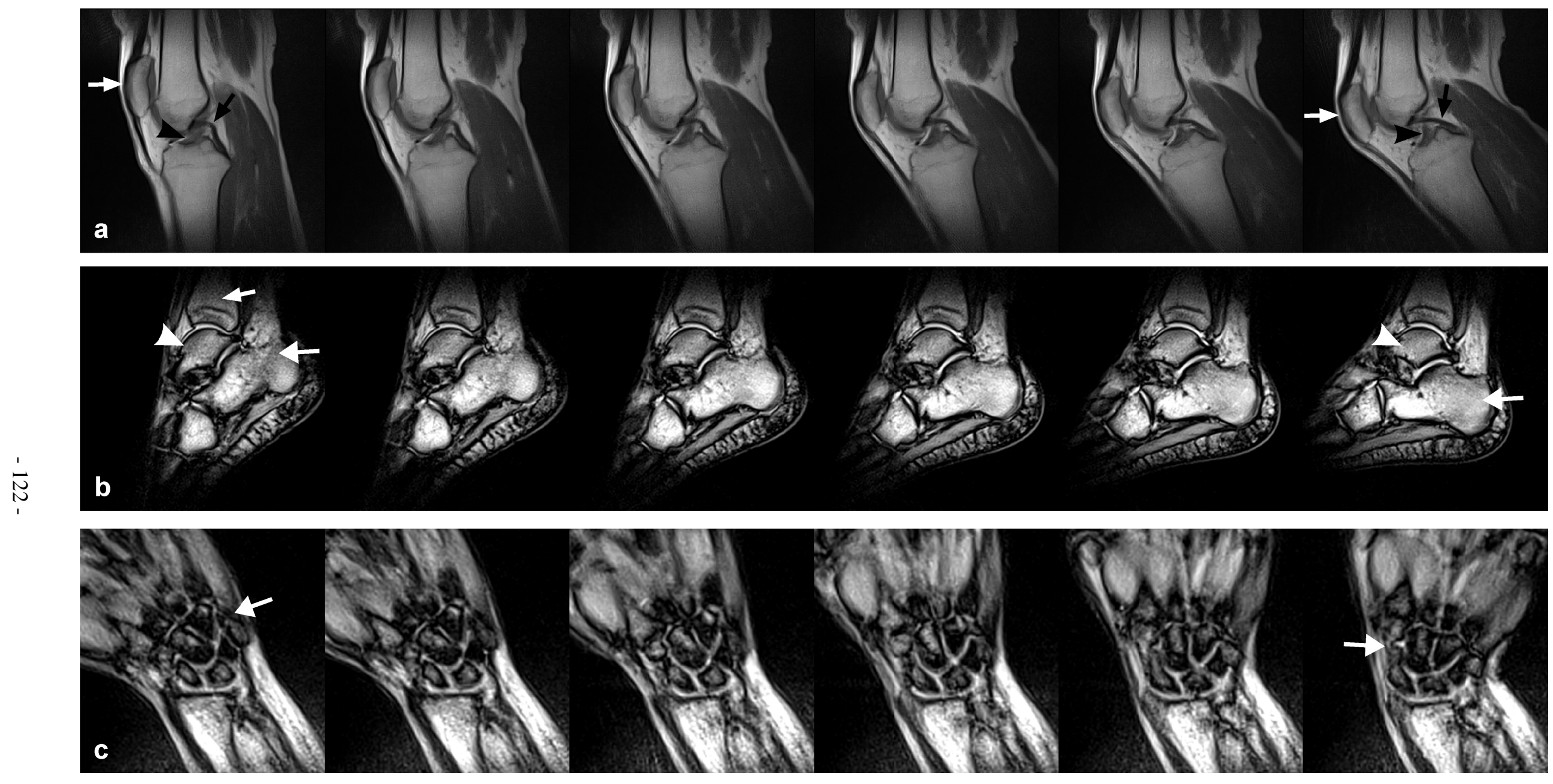

Figure 7.6 Radial FLASH RT-MRI during (a) flexion of the knee with in-phase contrast, (b) tibiotalar rotation of the ankle with opposed-contrast, and (c) radialulnar deviation of the wrist with opposed-phase contrast. White arrows in (a) indicate patellar displacement relative to the femur, the anterior cruciate ligament (black arrow heads), and the posterior cruciate ligament (black arrows); white arrow head and white arrow in (b) indicate the talus and calcaneus, respectively, relative to the tibia (small white arrow); arrow in (c) indicates the carpal bones and intercarpal spaces. The imaging parameters: radial refocused FLASH, FOV $192 \times 192 \mathrm{~mm}^{2}$, matrix size 256×256, in-plane resolution $0.75 \times 0.75 \mathrm{~mm}^{2}, S L T 5 \mathrm{~mm}, T R / T E 4.33 / 2.3$ (in-phase) or 5.63/3.5 (opposed-phase), FA $30^{\circ}$ (in-phase) or $25^{\circ}$ (opposed-phase), $B W 810 \mathrm{~Hz} / \mathrm{Pixel}$, number of views 385 (in-phase) or 295 (opposed-phase), effective temporal resolution $3 \mathrm{~Hz}$. 
knee (healthy volunteer, male, 30 years old) without resistance to stress in a mid-sagittal plane with in-phase contrast was accomplished by using a small flexible coil (Siemens AG, Erlangen, Germany). The results are shown in Fig 7.6a. Tibiotalar rotation of the ankle (dorsiflexing and plantarflexing, associated with an low degree external and internal talar rotation) in a mid-sagittal plane (healthy volunteer, female 27 years old) with opposed-phase contrast was acquired with the use of a CPC coil (Noras MRI Products, Höchberg, Germany) and the results are shown in Fig 7.6b. Radial-ulnar deviation of the wrist in the coronal plane (same volunteer as in Fig 7.6b) with opposed-phase contrast using a small flexible coil is shown in Fig 7.6c.

During the experiment, the subject was placed in a prone (knee and ankle measurement) or supine (wrist measurement) position with the initial limbs extended. Foam padding was placed at various sites for support and comfort. Noticeably, the position and morphological changes of the interested anatomical structure, e.g. carpal bones as well as intercarpal spaces in Fig 7.6c, were all clearly presented through the dynamic imaging.

The results illustrate the usefulness of the proposed radial FLASH RT-MRI for functional evaluation of the musculoskeletal kinematics, for diagnosis of pathological conditions, for example, soft tissue impingement syndromes and abnormalities such as tendon subluxation, and also for therapeutic assessments during rehabilitation.

\subsection{Speech Production}

Human speech arises from orchestrated activities of phonatory and articulatory organs and reflects human-specific characteristics in anatomy and physiology. From a technical point of view, the production of speech is described as a two-stage process [321, 322]. In the first stage the sound or human voice is initiated in the lung by producing adequate airflow and air pressure, while in the second stage it is further filtered by the vocal tract, which consists of the laryngeal cavity, the pharynx, the oral cavity, and the nasal cavity. Specifically, the vocal folds (vocal cords), which are a vibrating valve and chop up the airflow from the lungs into audible pulses, in combination with the articulators (tongue, palate, cheek, lips, etc.), which interact with the laryngeal airflow, are capable of producing distinct and highly intricate sounds. These biological specificities provide aerodynamic and acoustic bases of speech production and contribute to generating a parallel time-pattern of gradually changing vocal signals with ripples in amplitude and spectrum due to rapid articulatory movements [323]. 
Understanding human speech production is of great interest from phonetic, linguistic, engineering, and several other research aspects. So far, many approaches have been used to obtain relevant information, for example, electropalatography (EPG) or electromagnetic articulography (EMA) examining linguapalatal contact and ultrasound for examining tonguesurface contours. But none of them yields real-time movies of articulators along the entire length and diameter of the vocal tract. Although video fluoroscopy technique does permit this, it involves $\mathrm{X}$-ray radiation and is thus limited in use.

Here the proposed real-time MRI method is applied to investigate the rapid and complex movements of the vocal tract.
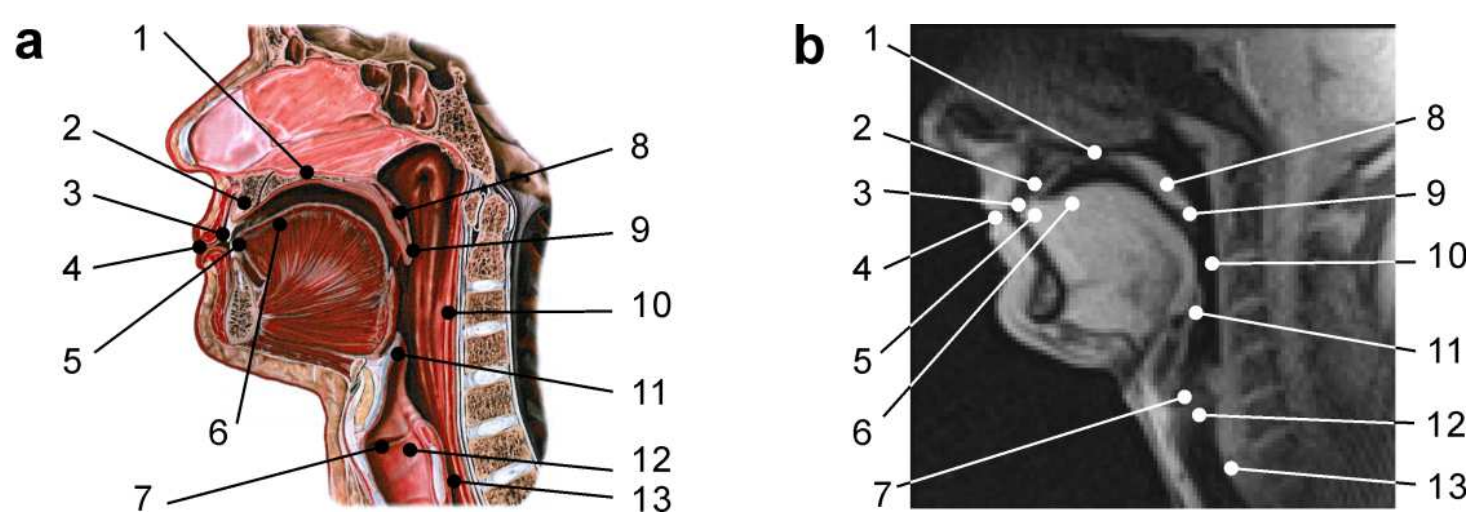

Figure 7.7 Anatomy of the throat. (a) Schematic illustration (modified from [324]). (b) Image acquired by using the spoiled radial FLASH RT-MRI technique.
1. Hard palate
6 . Blade of the tongue
10. Pharynx
2. Teethridge
7. Vocal ford
11. Epiglottis
3. Teeth
8. Soft palate
12. Trachea
4. Lips
9. Uvula
13. Esophagus
5. Tip of the tongue

During the examination, a mid-sagittal image of the head is acquired. The vocal tract from the vocal folds (glottis) to the lips is covered as illustrated in Fig 7.7b. In this image, the air-tissue boundaries of the anatomical components that are of interest as shown in Fig 7.7a are represented and can be traced during the production of speech. The imaging parameters were: spoiled radial FLASH, FOV $192 \times 192 \mathrm{~mm}^{2}$, matrix size $112 \times 112$, in-plane resolution $1.7 \times 1.7$ $\mathrm{mm}^{2}$, slice thickness $10 \mathrm{~mm}, T R / T E 2.2 / 1.5 \mathrm{~ms}, F A 8^{\circ}, B W 1540 \mathrm{~Hz} / \mathrm{Pixel}$, number of views 93 with 3 interleaves, effective temporal resolution $15 \mathrm{fps}$. A small flexible coil in front of the subject's fact and a 4-cm diameter surface coil attached to subject's neck were used (Siemens AG, Germany). It can also be seen from this image that the improved acquisition technique with such a short $T E$ offers excellent robustness against magnetic susceptibility differences between air and human tissue, which is a typical problem of the upper airway imaging. 

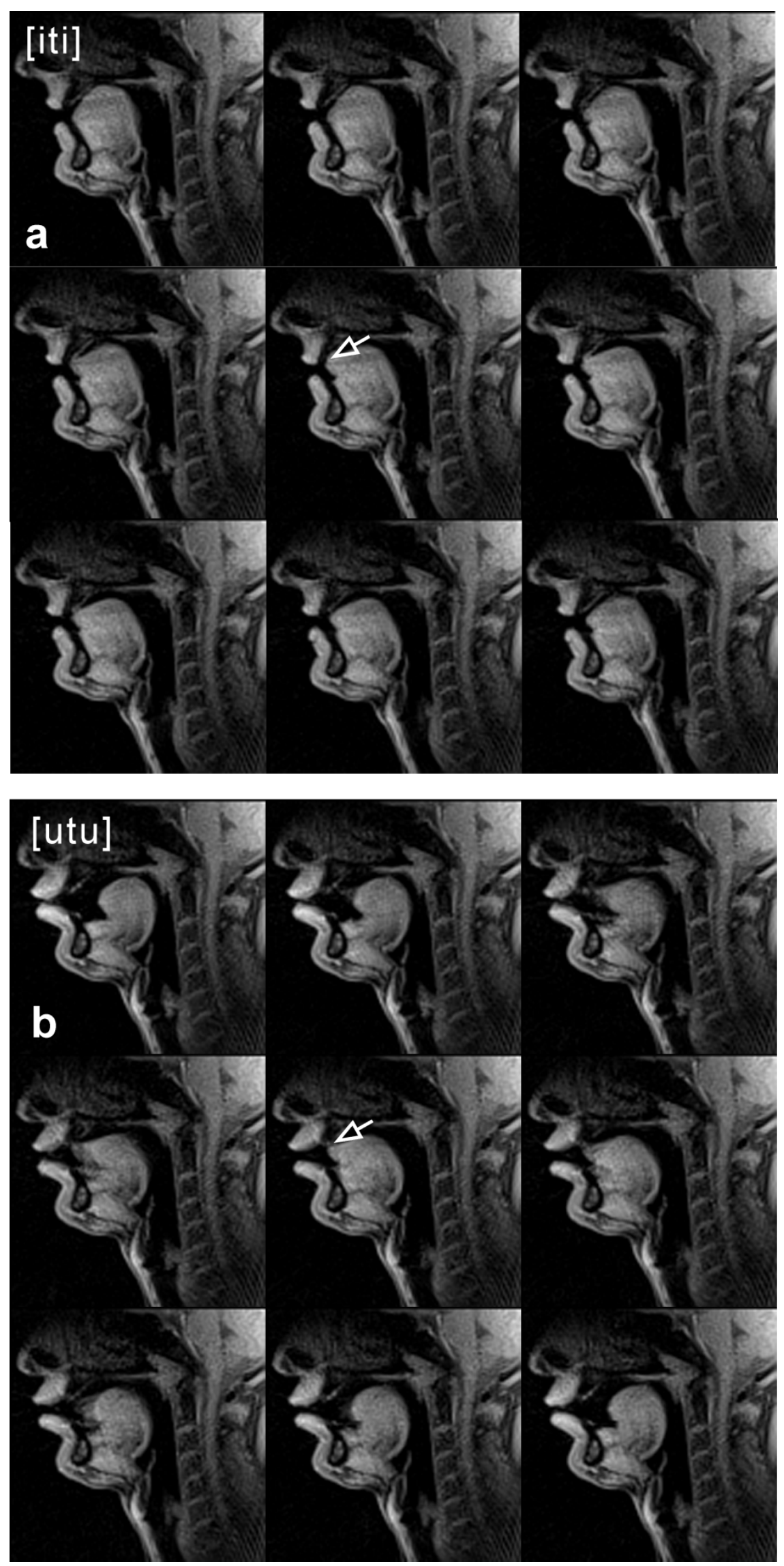

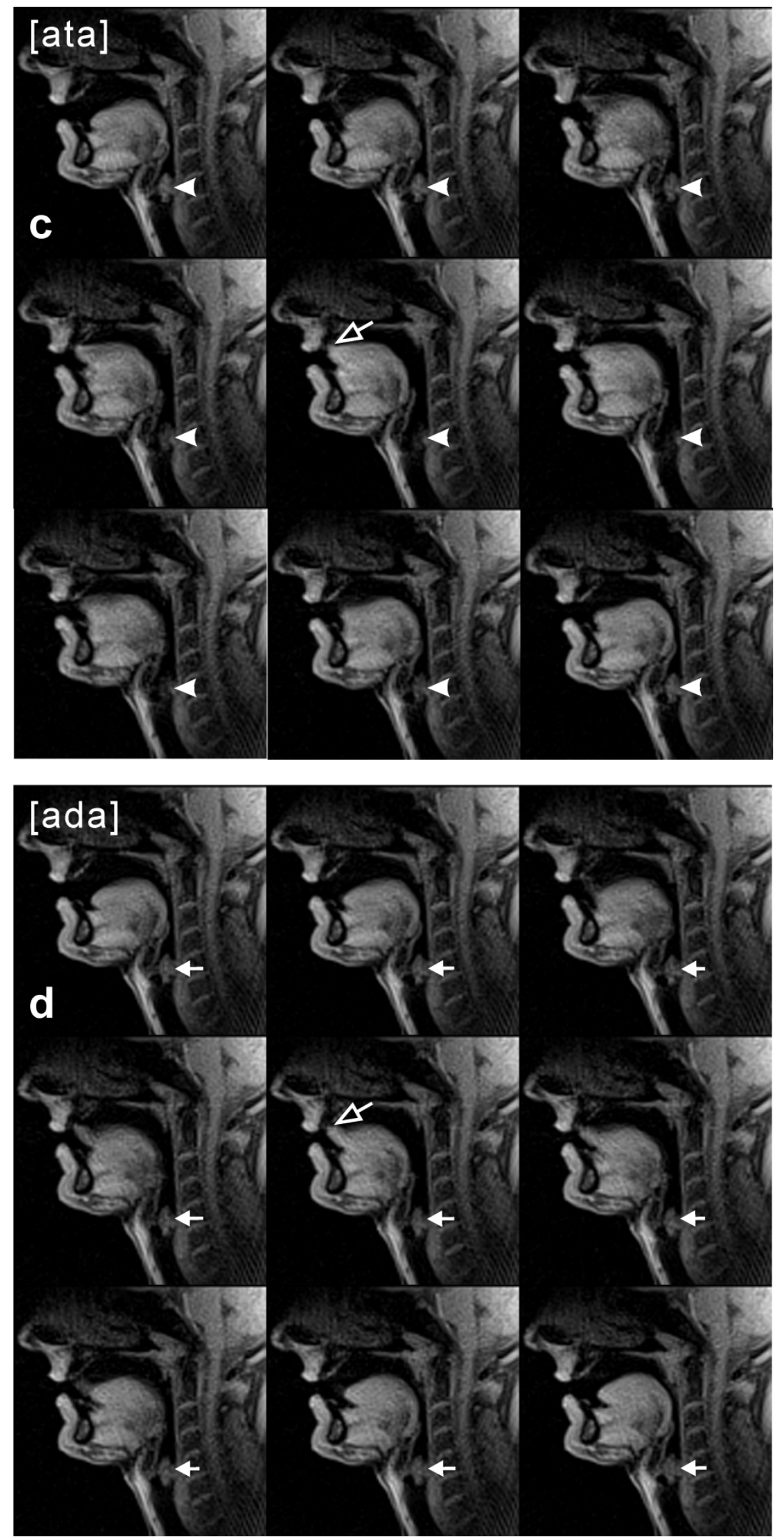

Figure 7.8 Radial FLASH RT-MRI of speech production with logatom words. Experiment was done with a healthy volunteer (male, native German, 24 years old) in a middle sagittal plane. Details of the figure and the arrow indications are in the text. 
Two experiments were performed. In the first experiment, a sequence of meaningless logatom words adapted from [325] was used to study the coarticulation effect of a vowel with an adjacent consonant. Each logatom word was in a consonant-vowel-consonant-vowel (CVCV) structure, all beginning with the consonant $b$. Three vowels ([a], [i], [u]) and 12 consonants were tested. Fig 7.8 shows an example of four logatom words "biti", "butu", "bata", and "bada" (healthy volunteer, male, 24 years old). The presented frames in each row were nine consecutive images from measurement of each individual word. They covered the whole transition period from the vowel to the consonant and to the vowel again, e.g. [iti] as directly indicated in the figure, with the middle one (i.e. $5^{\text {th }}$ frame) representing the exact time point of the production of the consonant $[\mathrm{t}]$ or $[\mathrm{d}]$.

When comparing [iti], [utu], and [ata] (i.e. Fig 7.8 a, b, and c correspondingly), the position of the tongue differed remarkably in the first or the last two frames, due to the pronunciation of three different cardinal vowels. For example, [i] is produced with the tongue as far forward and as high in the mouth as is possible; $[\mathrm{u}]$ is produced with the tongue as far back and as high in the mouth as is possible, with pursed lips; [a] is produced with the tongue as far back and as low in the mouth as possible. This can also be captured in the static MRI studies. During the production of the consonant $[t]$ in each word (comparing within the same row), the shape of the lips hardly changed. This is because, as an alveolar plosive, the shape of the lips has no influence on the production of the $[t]$. However, the movement of the tongue was obvious, particularly, the raised tip and the blade of the tongue against the alveolar ridge (hollow white arrows), which helped to produce the sound of [t]. Comparing Fig 7.8c and d, although both $[\mathrm{t}]$ and $[\mathrm{d}]$ are alveolar plosives and thus no big difference were seen in the formation of the lips and the tongue, as a voiceless alveolar plosive, [t] requires opening of the vocal folds to allow the production of the sound - represented by the decreased intensity of the larynx from [a] to [t] which increased again from [t] to [a] (white arrow heads), also observable in Fig 7.8a and b. On the contrary, as a voiced alveolar plosive, [d] is produced with vibrations of the vocal cords - represented by the high intensity of the larynx (white arrows). These are the specific features provided by the RT-MRI technique which cannot be obtained by the static or pseudo-dynamic studies.

In the second experiment, the test material included real German words also with CVCV structures, in a context of consonant-[a]-consonant-[ə], to study the consonant articulation. In these chosen words, most of the lingual consonants of the Standard Modern German (Neuhochdeutsch) are represented. The details may be found in [326]. Fig 7.9 shows an example of 10 consonant articulations from a native German (male, 24 years old). For the 


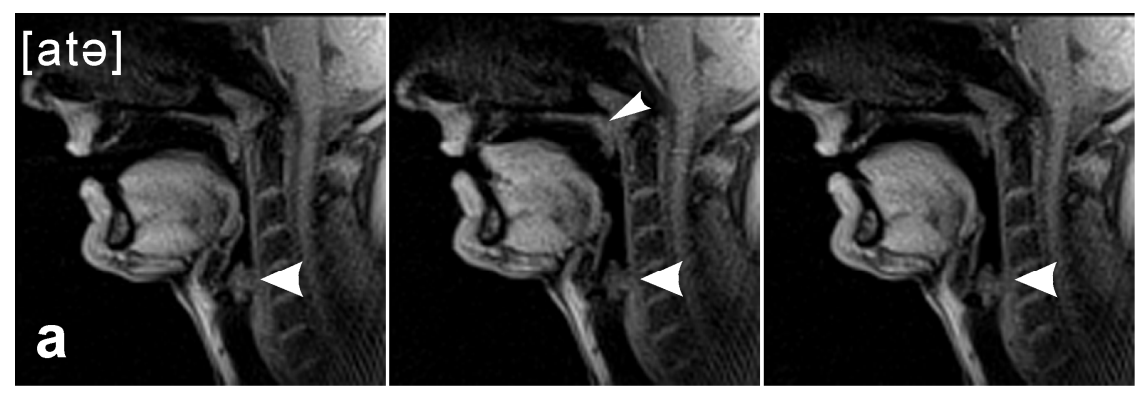

- alveolar

- plosive

- voiceless

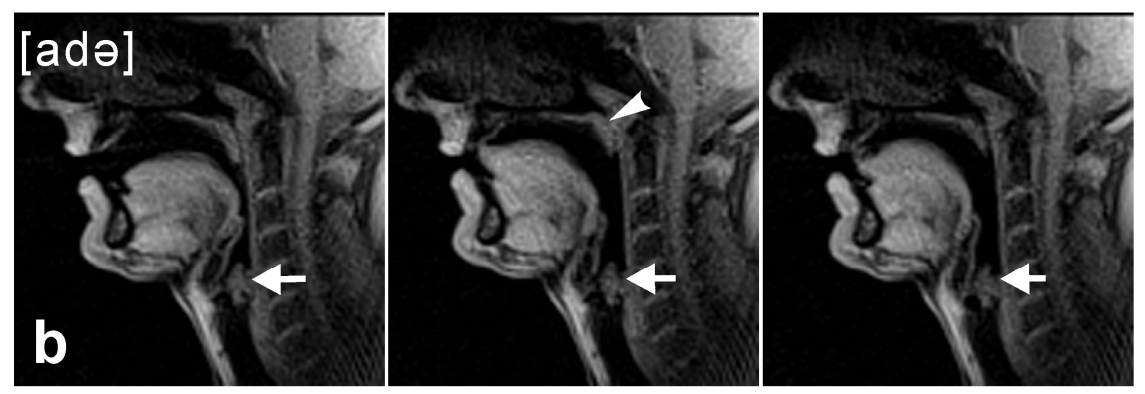

- alveolar

- plosive

- voiced

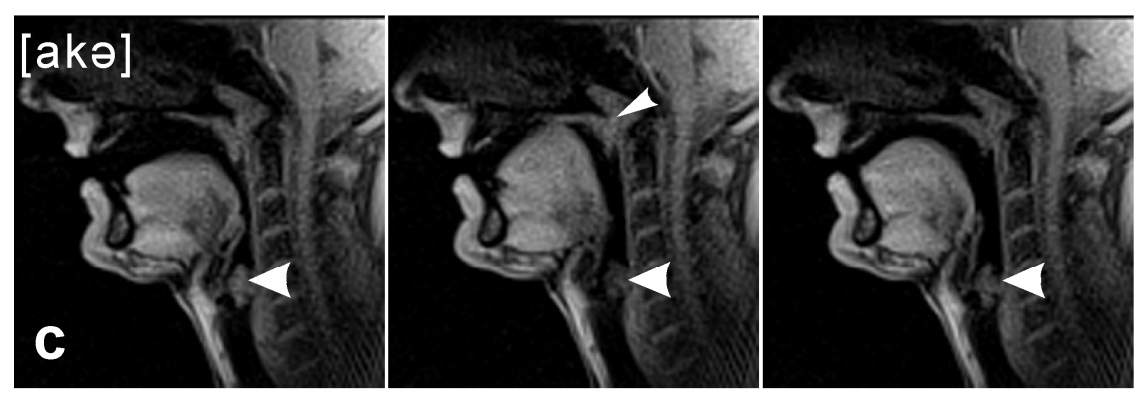

- velar

- plosive

-voiceless

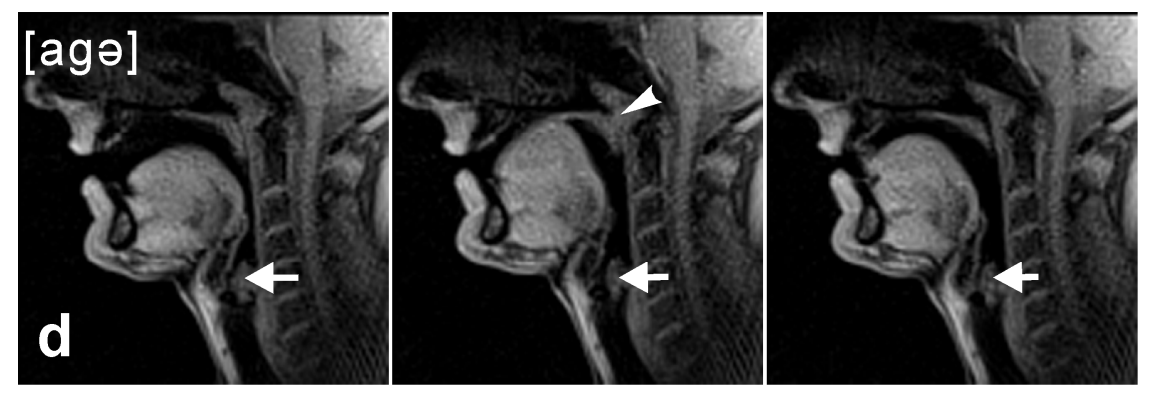

- velar

- plosive

- voiced

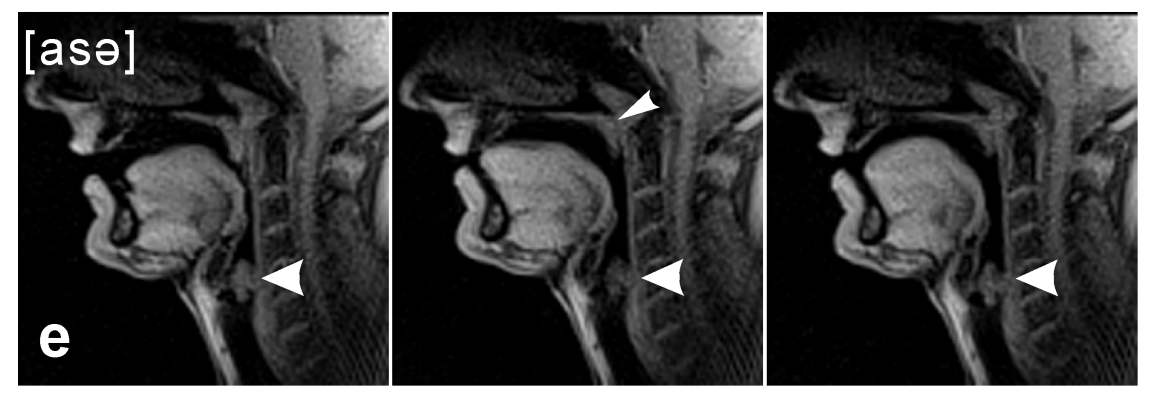

- alveolar

- fricative

- voiceless

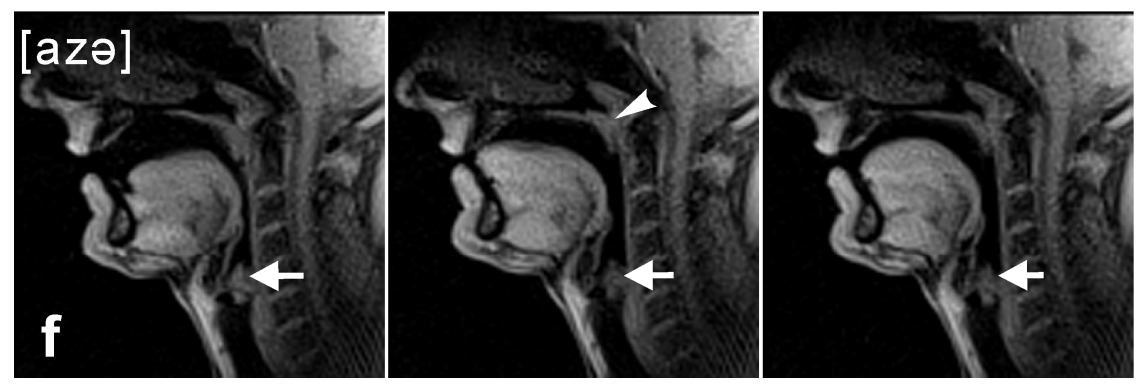

- alveolar

- fricative

- voiced 


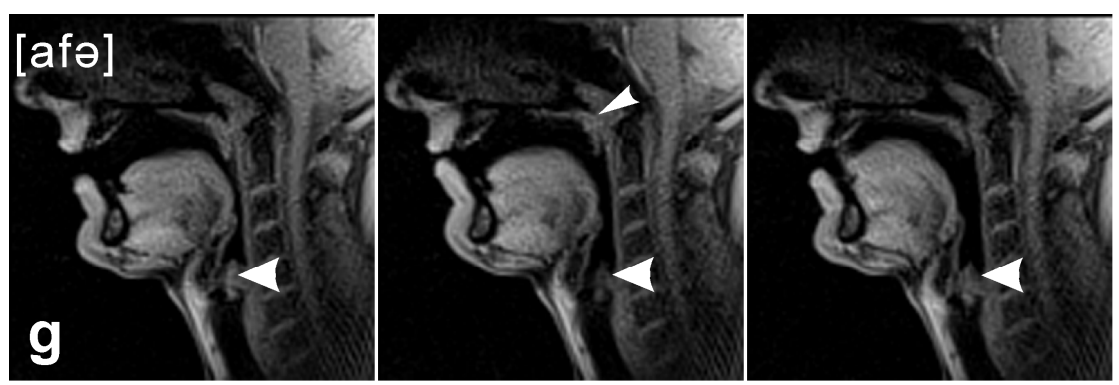

- labiodental

- fricative

- voiceless

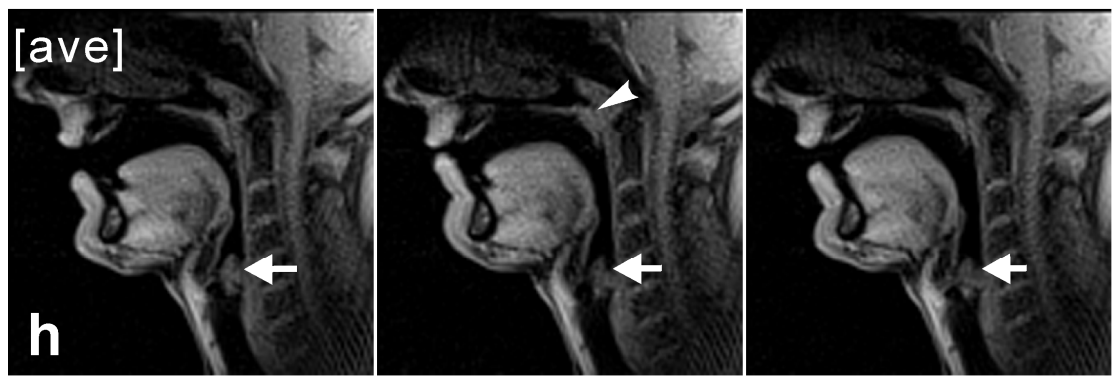

- labiodental

- fricative

- voiced

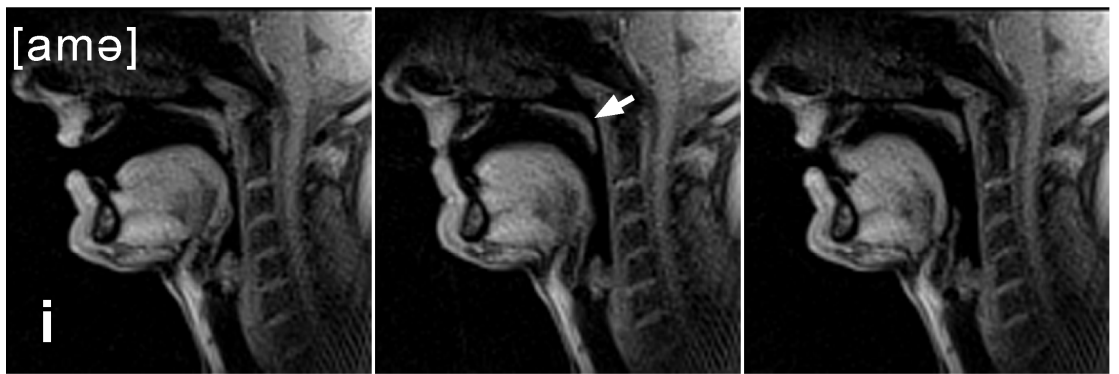

- bilabial

- nasal

- voiced

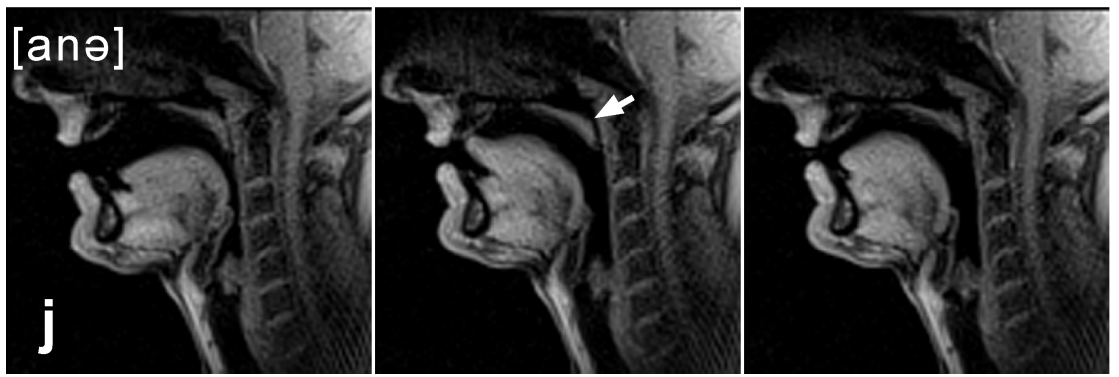

- alveolar

- nasal

- voiced

Figure 7.9 Radial FLASH RT-MRI of speech production with German words. Same volunteer and imaging parameters as that in Fig 7.8. Text to the right side of the images indicate the area of articulation, manner of articulation, and whether the consonant is voiceless or voiced, respectively. Details of the fig and the arrow indications are in the text.

purposes of demonstration, the presented images in each row were all chosen with about 300 ms time interval (4-frame interval), so that the middle image represents the production of the corresponding consonant, while the first and last images represent the production of [a] and [ə], respectively.

First, when comparing voiceless and voiced consonants independent of the articulation manner (i.e. whether plosive or fricative), i.e. $[\mathrm{t}],[\mathrm{k}],[\mathrm{s}],[\mathrm{f}]$ and $[\mathrm{d}],[\mathrm{g}],[\mathrm{z}],[\mathrm{v}]$, the slightly decreased intensity of the larynx from the first image to the second, which increased again from the second image to the third in the voiceless case, is observable (white arrow heads).

This indicates that during the production of the voiceless consonant, the vocal folds in the 
larynx are apart and air is able to flow quite freely from the lungs into the mouth cavity. On the contrary, the almost unchanged intensity in the voiced case (white arrows) indicates that the vocal folds are in a position close together to each other. Provided the air pressure in the lungs is sufficient, the vocal folds will be caused to vibrate against each other, in order to produce voiced consonants, as also demonstrated and described in the first experiment above. Second, depending upon different articulation places, the formation of the tongue and lips differed remarkably. When the articulation place is alveolar, as in the case of $[\mathrm{t}],[\mathrm{d}]$ and $[\mathrm{n}]$, the consonants are articulated with the tongue tip or the blade against the alveolar ridge (teethridge); when the articulation place is velar, as in the case of $[\mathrm{k}]$ and $[\mathrm{g}]$, the consonants are articulated with the back part of the tongue (the dorsum) against the soft palate (the velum); when the articulation place is labiodental, as in the case of [f] and [v], they are articulated with the lower lips and the upper teeth; when the articulation place is bilabial, as in the case of [m], it is articulated with both lips. It should be noticed that, due to the extremely short T2 of the teeth, the touching of the lower lips and the upper teeth in the labiodental case, as well as the narrowing of the upper and lower teeth to constrict air flow in the fricative case, is not well recognized.

Third, the open of the nasal cavity through the soft palatal (small white arrows) in the case of nasal consonant, as in [m] and [n], indicates that the air is allowed to escape through the nose to produce the sound. This is a distinct feature in comparison to the oral consonant, as in the other cases shown in the figure, the soft palatal is seen in contact with the pharynx (small white arrow heads) so that the air is allowed to escape through the mouth.

The above examples demonstrate the capability of the radial FLASH real-time MRI in offering excellent spatiotemporal information about the coordination of articulator configurations and the shape changes of the vocal tract. Although MRI has already been applied in various studies of speech production, either in a cine mode $[327,328]$ or in a true dynamic manner [329-331], either in studying the vowels with static imaging [332, 333] or also the stop consonants with signal triggering [334], the present preliminary results generally show superior image quality. Moreover, they also permit quantitative analyses and modeling of the time-varying vocal tract shape for the purpose of gaining deeper understanding of the underlying principles that govern the speech production process. Further technical developments may include visualization of the teeth and simultaneous noise-robust audio recording during real-time MRI. 


\subsection{Swallowing Process}

Swallowing, known scientifically as deglutition, normally occurs as an orderly physiologic process that transports ingested material and saliva from the mouth to the pharynx, the esophagus, and into the stomach $[335,336]$. Although the majority of swallowing happens subconsciously in response to salivation, which occurs at about $0.5 \mathrm{ml} / \mathrm{min}$ initiating spontaneous swallowing at about 1/min [337] or about 1000 swallows per day in awake subjects [338], it is generally considered to be voluntary and is evoked with conscious input (e.g. during eating). The whole swallowing sequences are complex neuromuscular activity involving both skeletal muscle (tongue) and smooth muscles of the pharynx and esophagus.

Earliest MRI study of the act of swallowing and tongue movements was based on the realization of the Cartesian FLASH technique [339] with gadolinium-diethylenetriamine pentaacetic acid (Gd-DTPA) as a contract agent. By reducing the phase-encoding steps, an effective temporal resolution of 5 fps was achieved. However, the obtained images had the very low spatial resolution of $3.0 \times 3.0 \mathrm{~mm}^{2}$ and were significantly degraded by motion artifacts. Later on, with the development of other rapid pulse sequences in clinic, the EPI and turbo-FLASH techniques became the most used methods in swallowing MRI studies [340345], with spatial resolution at about $3.0 \times 3.0 \mathrm{~mm}^{2}$ and temporal resolution ranging from $3 \mathrm{fps}$ to 15 fps. However, the obtained images still suffered from distortion induced by the physiological motion, either from the anatomical structures which are involved in the act of swallowing, or from pulsatile blood flow, or even from concurrent head movement during swallowing. Some recent studies employed the single-shot FSE technique [346, 347]. But due to long acquisition time (700 ms) which was just slightly less than the whole duration of one swallow process (1-1.5 s), only one or two images could be obtained per swallow. Therefore, the swallowing process had to be repeated many times (e.g. at least 10 times) in order to acquire multiple images. During post-processing, each image was sorted retrospectively according to its moment in the swallowing cycle and the whole image series was played in the cine mode to give a "dynamic" view. Although such a pseudo-dynamic method in this case does reflect the true physiological process, compared to the similar approach in TMJ studies with bite device, the imaging time is prolonged. Moreover, because the images are acquired during different swallows, an analysis of the timing of swallowing events was not possible.

In this thesis, the proposed RT-MRI is applied to study the process and effect of swallowing, particularly, the movement changes of the larynx and the pharynx during 
swallowing. Two experiments were performed: dry swallowing with saliva and swallowing of pineapple juice as an oral contrast agent, which has been proved to offer the best positive contrast in $\mathrm{T} 1 \mathrm{~W}$ images due to the high concentration of the paramagnetic substance (i.e. manganese) by previous studies [348]. This observation was confirmed by the experiments during this work in comparison to water, milk and other fluids. For both studies, a small flexible coil (Siemens AG, Erlangen, Germany) was used in front of the subject's lower face. For the swallowing study with pineapple juice, a plastic infusion tube was used with one end connected to the subject's mouth and the other connected to a plastic bag placed outside the magnet bore at about the same level as the examination bed, so that the subject can freely adjust the flow volume and speed. During real-time imaging, the subjects were instructed to swallow in a natural manner and with a comfortable rate and bolus volume continuously. One single slice is chosen in the mid-sagittal plane, as shown in Fig 7.7b in the previous section, covering the area of interest including the mouth, pharynx, larynx and the upper part of the esophagus.

Fig 7.10 shows the results of a swallowing study with pineapple juice from a healthy volunteer (female, 34 years old). The imaging parameters were: spoiled radial FLASH, FOV $220 \times 220 \mathrm{~mm}^{2}$, matrix size $128 \times 128$, in-plane resolution $1.7 \times 1.7 \mathrm{~mm}^{2}$, slice thickness $10 \mathrm{~mm}$, TR/TE 2.1/1.3 ms, FA $8^{\circ}, B W 1560 \mathrm{~Hz} / \mathrm{Pixel}$, number of views 93 with 3 interleaves, TA/TU 195/65 ms, effective temporal resolution 15 fps. Fig 7.10 a, b, c and d represent four physiological phases that the whole swallowing process is normally divided into: preparatory, oral, pharyngeal and esophageal phase. Due to ultra-fast movement in the pharyngeal phase, the three images in Fig 7.10c were chosen with $130 \mathrm{~ms}$ time interval, while the images in the other phases were chosen with about $250 \mathrm{~ms}$ time interval. First, in the preparatory phase, the bolus was taken into the oral cavity and masticated to prepare for swallowing. Second, in the oral phase, the bolus was propelled from the oral cavity into the pharynx by voluntary tongue movement. Third, in the pharyngeal phase, several involuntary and synchronous actions were involved, including palatal closure to prevent nasal reflux (white arrows), glottal closure with larynx elevation to prevent aspiration (white arrow heads), esophageal opening (small white arrow) and bolus transport into the esophagus. The pineapple juice gave very bright signal in the above three phases and offered excellent contrast to the anatomical tissues. Fourth, in the esophageal phase, the function-related anatomical structure (i.e. soft palate, tongue, pharynx and hyoid bone) were back to the original position and the bolus was hardly visible due to compressed contrast agent and low coil sensitivity. 

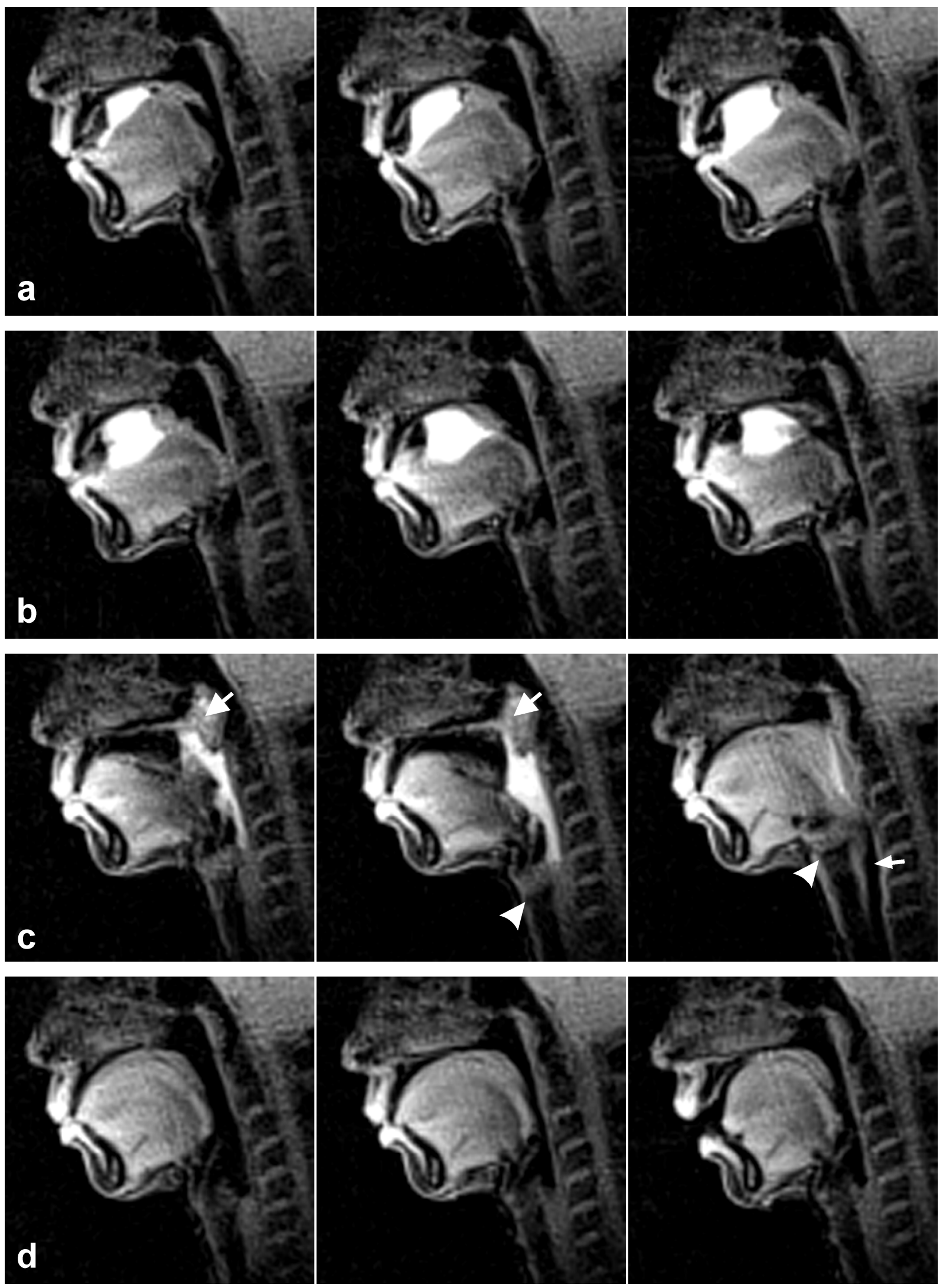

Figure 7.10 Radial FLASH RT-MRI of the swallowing process. Images were acquired from a healthy volunteer (female, 34 years old) in a middle sagittal plane. (a, b, c, and d) represent the four physiological phases of the whole swallowing process: preparatory, oral, pharyngeal, and esophageal phase. High intensity areas in (a, $\mathbf{b}$, and $\mathbf{c})$ indicate the pineapple juice as the contrast agent. Details of the imaging parameters and the arrow indications are in the text. 
The results demonstrate the feasibility and effectivity of the developed radial FLASH RT-MRI in investigating swallowing functions and morphology. Extension to the other imaging planes, e.g. in oblique coronal plane to study deglutitive vocal fold motion, is possible. It is also expected to be of value in better understanding the swallowing mechanism of the collaborative work among the anatomical apparatus and in clinical diagnosis and evaluation of patients with oropharyngeal pathology or complex disorders such as aspiration and dysphagia.

\subsection{Cardiovascular Motion}

MRI of cardiovascular structures provides a noninvasive method of viewing the heart and major vessels. It is always of great interest, because it serves as a very important tool in clinical diagnosis for evaluating conditions such as heart failure and congenital heart disease, as well as for determining the damage caused by a heart attack or progressive heart disease. Recent progress in MRI techniques [19, 205], for example in fast imaging techniques such as parallel imaging [234] and non-Cartesian imaging [254, 349] or in black blood imaging techniques [85, 350], has led to a rapid increase in the number of clinical applications. At the same time, MRI also always faces the challenge from the rapid and complex movement of the anatomical structures that are to be imaged. Commonly in cardiovascular MRI, the problem of cardiac motion is addressed by synchronizing the MR measurements with the electrocardiogram (ECG) [62], while the respiratory motion is controlled by performing breathhold [351] or placing a respiratory navigator on the diaphragm for movement tracking $[352,353]$. However, this causes several new problems and becomes a current limitation of cardiovascular MRI. First, both cardiac and respiratory motion compensation techniques can suffer from inaccuracies. ECG triggering is susceptible to radiofrequency and gradient interferences, which may lead to trigger detection problems, while extracting the displacement data from respiratory navigators may not reflect motion of the heart accurately. In addition, image acquisition with ECG synchronization often encounters problem for patients with arrhythmia. Second, patients with cardiac diseases frequently have difficulty sustaining repeated, long breathholds, or even one breathhold of 10 to $20 \mathrm{~s}$. In addition, repeated breathhold can yield poor image quality due to inconsistency, while extended breathholds may lead to changes in flow and pressure in the region of the heart. Third, placing ECG electrodes and handling the connections as well as the need for breathhold increase the complexity of 
cardiac imaging and reduce patient comfort. Other approaches to overcome these problems employ self-gating techniques that extract the motion data for measurement triggering or gating directly from the acquired MR signals, mostly suited in a retrospective manner [354356] and in a few cases also in a prospective way [357]. However, while the sophisticated algorithms have been used, resolving cardiac motion with high effectivity remains difficult to achieve. In addition, in most of the techniques, segmented $k$-space acquisition requires retrospective reconstruction and display offline after the whole examination. For all these approaches, the entire imaging time is prolonged either because the signal acquisition time is constrained to specific window (as in breathhold, respiratory or ECG gating cases) or because many acquired data points are discarded in reconstruction depending on the gating and filtering algorithm (as in the self-gating case). Therefore, continuous MR imaging of the cardiovascular system for multiple cardiac phases without breathhold or any synchronization and with simultaneous online image display is highly demanded.

Examples of the proposed RT-MRI method in cardiac imaging have already been shown in Fig 4.10, Fig 6.4, Fig 6.5, and Fig 6.7 of the previous chapters, with a series of consecutive images acquired without ECG synchronization and breathhold, making such examinations feasible in a greater number of patients. Fig 6.6 even demonstrates that real-time cardiovascular imaging could be accomplished with update times (TU) of $30 \mathrm{~ms}$ per image corresponding to a frame rate beyond those commonly in the clinical ultrasound examinations, and even below - with $T U$ of $14 \mathrm{~ms}$ (acquisition times $70 \mathrm{~ms}$ per image). More examples from systematic studies are shown in Fig 7.11, all with standard clinical image plane orientations. These images represent spoiled radial FLASH acquisitions with a 32-channel body array coil (Siemens AG, Erlangen, Germany) at the shortest possible $T R$ and with moderate undersampling and update time. The detailed imaging parameters were: FOV $256 \times 256 \mathrm{~mm}^{2}$, image matrix size $128 \times 128$, in-plane resolution $2 \times 2 \mathrm{~mm}^{2}$ with $8 \mathrm{~mm}$ slice thickness, TR/TE 2.02/1.30 ms, FA $8^{\circ}, B W 1950 \mathrm{~Hz} / \mathrm{Pixel}$, number of views 125 with 5 interleaves. While the acquisition time (TA) for a single image was $250 \mathrm{~ms}$, image updates were reconstructed every 25 interleaved spokes corresponding to an effective temporal resolution of $50 \mathrm{~ms}$ (20 fps). The selected images in each row cover half of the cardiac cycle from end-diastole to end-systole. The visualized anatomical structures, including cardiac chambers, valves, and vessels, are also indicated in the figure. 

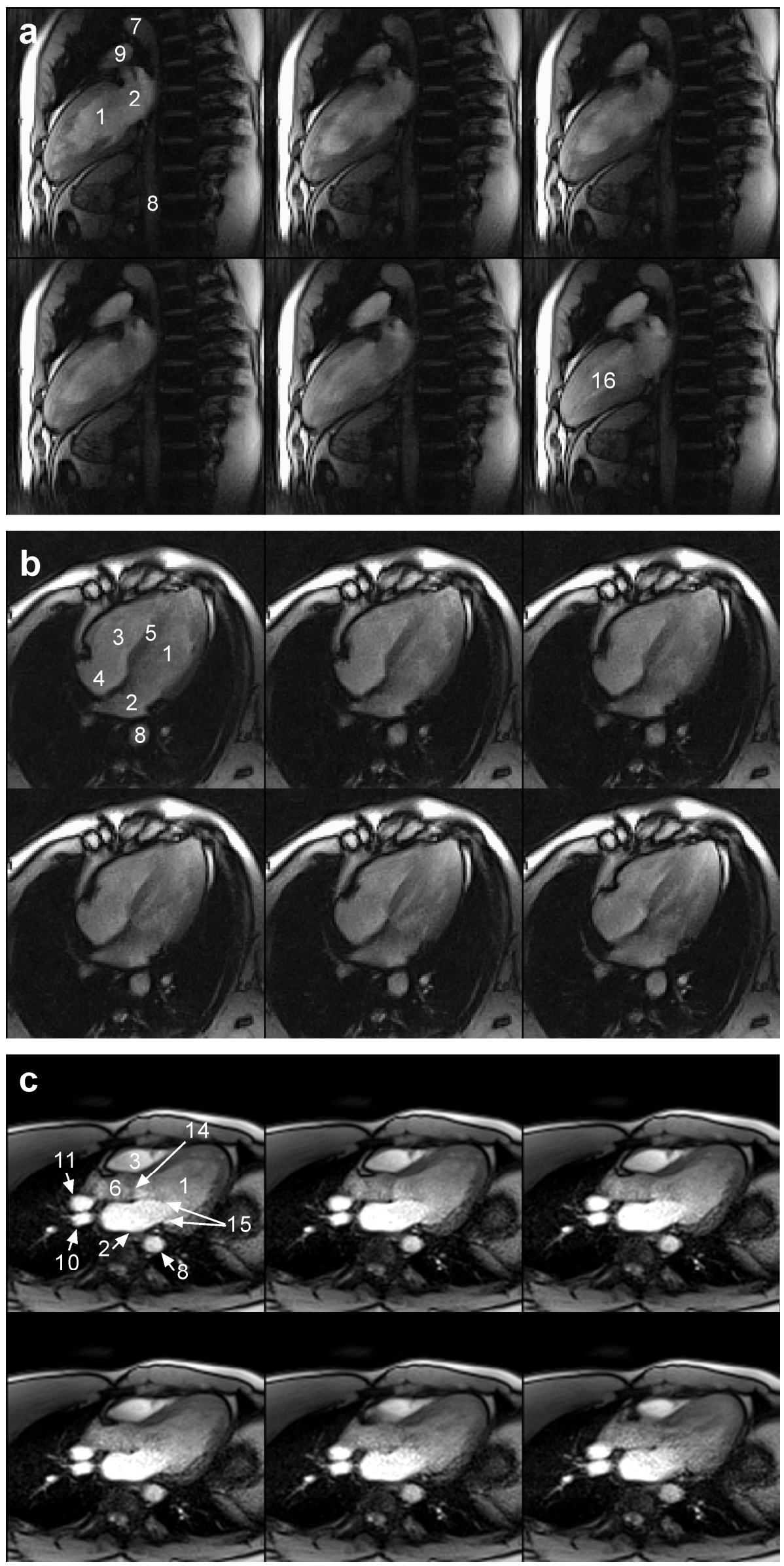

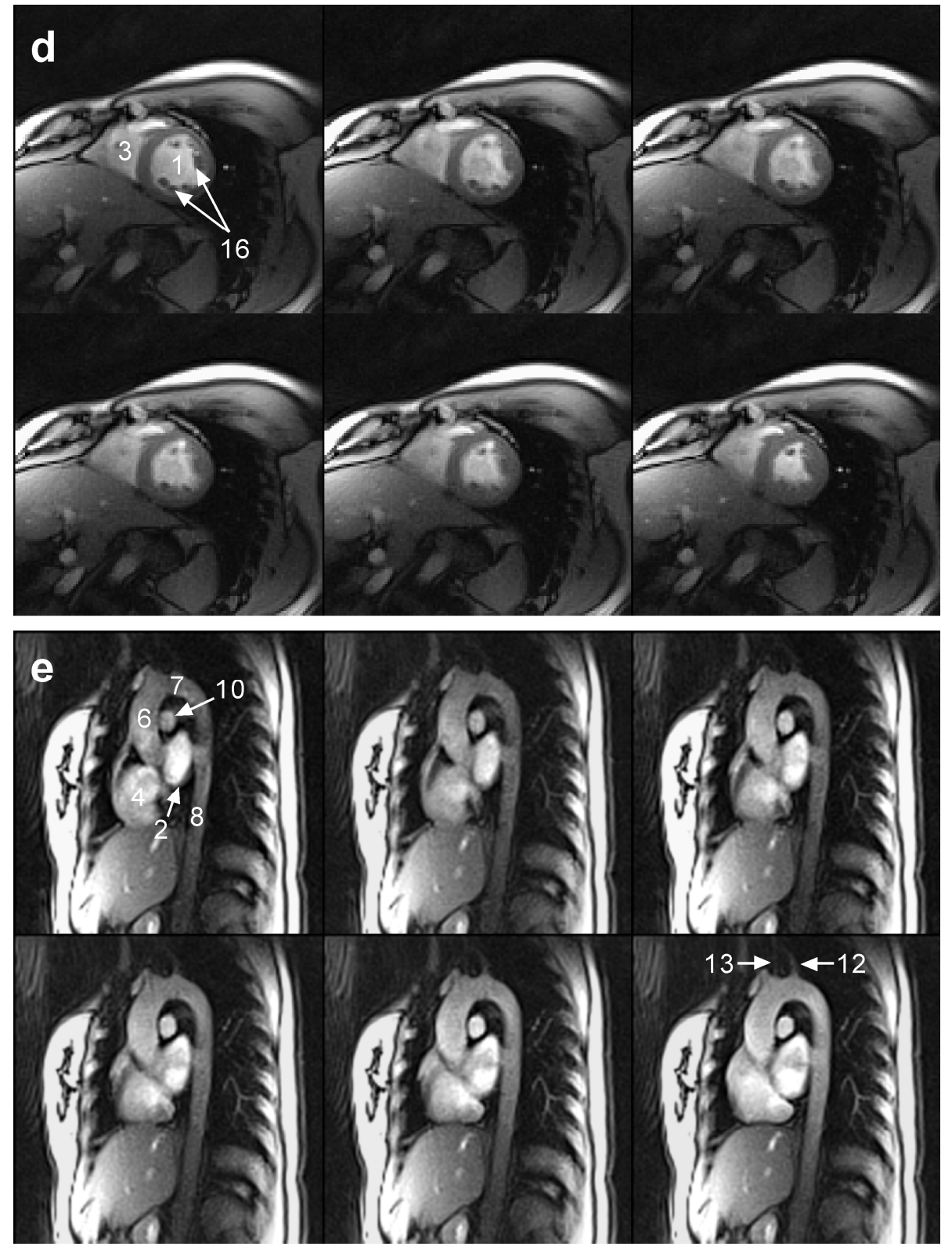

Figure 7.11 Radial FLASH RT-MRI of the cardiovascular system at $2.0 \times 2.0 \times 8 \mathrm{~mm}^{3}$ spatial resolution and 20 fps temporal resolution, in an anatomically defined (a) 2-chamber view, (b) 4-chamber view, (c) left ventricular inflow/outflow long-axis view, (d) short-axis view and (e) oblique plane through aortic arch or so-called "candy cane view". (a, b and d) were from one healthy volunteer (male, 28 years old); (c and d) were from another volunteer (female, 35 years old). The imaging parameters: spoiled radial FLASH, FOV $256 \times 256 \mathrm{~mm}^{2}$, matrix size $128 \times 128$, SLT $8 \mathrm{~mm}$, TR/TE 2.02/1.30 ms, FA $8^{\circ}, B W 1950 \mathrm{~Hz} / \mathrm{Pixel}$.
1. Left ventricle (LV)
2. Left atrium (LA)
3. Right ventricle (RV)
4. Right atrium (RA)
5. Interventricular septum
6. Ascending aorta
7. Aortic arch
8. Descending aorta

9. Main pulmonary artery (MPA)

10. Right pulmonary artery (RPA)

11. Superior vena cava

12. Left subclavian artery

13. Left common carotid artery

14. Aortic valve

15. Mitral valve

16. Papillary muscles 
Normally, in cardiac MRI, best results are obtained at late diastole, whereas very rapid myocardial wall movements during late systole yield unacceptable image distortions, particularly in anatomical planes other than short-axis view due to the complex structures and movements. In contrast, the results presented here demonstrate the effectivity of the radial FLASH RT-MRI in resolving the cardiovascular motion during the whole cardiac cycle.

All these preliminary results presented above demonstrate clearly that the proposed radial FLASH RT-MRI technique offers an unique ability for the investigation of anatomical structures as well as the functional performance of the heart and thoracic great vessels. In addition, the absence of smearing and blurring facilitates the assessement of quantitative functional indexes, e.g. ventricular volumes, biventricular ejection fractions, biventricular stroke volumes, cardiac output, radial shortening, and systolic wall thickening in the analysis. 


\section{Chapter 8}

\section{Conclusion and Outlook}

\subsection{Conclusion}

Real-time magnetic resonance imaging (RT-MRI) is not a particular pulse sequence or image reconstruction algorithm but rather a technical approach and measurement strategy that markedly differs from conventional MRI. It prescribes, acquires, encodes, reconstructs, and displays images without noticeable delay. According to the physical nature of MRI, which involves a time-consuming acquisition process, a particular challenge of this thesis was to achieve both high spatial and temporal resolution, while maintaining a sufficiently high signal-to-noise ratio (SNR). This particularly refers to the monitoring of physiological processes, where small anatomical structures undergo significant movements. Despite the fact that rudimentary RT-MRI sequences have been available for some time, they do not offer a general solution for continuous online imaging and smooth monitoring with sufficient image quality and robustness.

My solution for RT-MRI as developed in this thesis is based on the following aspects:

(i) First, the FLASH technique is selected as the most suitable rapid imaging pulse sequence after experimental comparisons with alternative sequences. This choice is motivated by several advantages: the technique allows for both rapid and continuous MRI signal acquisition without compromising image quality, without limitations due to physical properties such as the duration of a relaxation time, and without safety problems due to excessive RF power deposition to the patient. For practical optimizations, the shortest acquisition times with adequate SNR and avoidance of artifacts or distortions is achieved by utilizing shortened RF excitation pulses, the shortest possible repetition time $(T R)$ and echo time $(T E)$, very low flip angles $(F A)$, and high receiver bandwidths $(B W)$. In addition, the variants of the FLASH technique, i.e. spoiled, refocused and balanced SSFP, which handle the residual magnetization in different ways, offer complementary image contrasts for applications to different tissues. Furthermore, variations of the gradient-echo time allow for in-phase and opposed-phase conditions emphasizing water and/or fat 
signals, which provide another contrast option particularly suitable for the abdomen and musculoskeletal system.

(ii) Second, the proposed method employs a radial $k$-space trajectory as an efficient spatial encoding scheme that, together with the physical properties of the FLASH technique, solves the conflict between spatial and temporal resolution. This selection is based on the advantageous property that the spatial resolution of the radial image is largely independent from the number of spokes, which makes radial acquisitions more tolerant to undersampling. The absence of a phase-encoding gradient avoids signal aliasing in the image (in-folding artifacts in Cartesian schemes, more complex distortions in spiral schemes) and the possibility of oversampling in all (radial) directions allows for unrestricted zooming. Even more importantly, the inherent oversampling in the central $k$ space area offers unique robustness against motion, which supports dynamic imaging. The only remaining problem stems from the sensitivity to phase differences between individual spokes that result from the overlap of all echo centers in the middle of $k$-space. Here, a suitable solution is presented that relies on a full $360^{\circ}$ coverage of $k$-space with an odd number of radial views and without oscillating gradient polarity, because in such a way, a symmetric distribution of susceptibility-induced phase errors is obtained over the entire $k$-space. At the same time, simple trajectory corrections are applied to compensate for hardware-related imperfections such as delays of gradient switching. Furthermore, a reordered interleaving strategy is developed to ensure a uniform distribution of spokes in $k$-space, which further reduces remaining motion inconsistencies by smearing out residual artifacts.

(iii) Third, image reconstruction is accomplished by gridding to a rectilinear data space followed by $2 \mathrm{D}$ inverse fast Fourier transform. With a proper choice of the interpolation window, radially encoded data are decoded and reconstructed into images, and are displayed with minimal temporal delay. In addition, the adaptation of sliding window and view sharing techniques decouples data acquisition and image reconstruction and allows for a further acceleration of image updates. Because each acquired view in the radial encoding scheme contains equivalent information covering both low and high spatial frequency components, its combination with interleaved radial sampling is ideally suited to achieve a smooth updating of the representation of moving objects.

The radial FLASH RT-MRI technique is implemented on a whole-body MRI system at 3T and experimentally validated on healthy human subjects. Though principally attractive for 
SNR reasons, the balanced SSFP variant is excluded for real-time imaging, because of its inherent sensitivity to magnetic field inhomogeneities and magnetic susceptibility differences. Most robust results were achieved using spoiled or refocused FLASH variants for RT-MRI. These latter methods resulted in high spatial and temporal resolution at the same time, a good SNR, and variable image contrast as demonstrated by a variety of biomedical applications. Proof-of-principle studies include a functional assessment of the temporomandibular joint as well as other joints of the musculoskeletal system with high (submillimeter) spatial resolution and relatively low temporal resolution ( 3 to $5 \mathrm{fps}$ ) as well as the monitoring of speech production and swallowing, and motions of the cardiovascular system with moderate spatial resolution (1.5 to $2 \mathrm{~mm}$ ) and high temporal resolution (even above $30 \mathrm{fps}$ ). These preliminary results indicate a tremendous potential of RT-MRI based on radial FLASH with a wide range of applications in both diagnostic imaging and biomedical research.

\subsection{Future Work}

The main technical issues that need to be addressed in future work are residual streaking artifacts that are related to severely undersampled radial encodings as well as the occurrence of temporal image blurring due to data sharing over measuring times that are relatively long compared to motional displacements.

First of all, streaking artifacts in radial RT-MRI arise from both undersampling and motion. Experiments in this thesis have shown that either increasing the number of views or slowing down the motion speed (if controllable) makes the streaking artifacts weaker in the image. On the other hand, moderate or even strong undersampling, as required for much shorter acquisition times for processes such as cardiac motions (Fig 6.8) or abrupt joint movements (Fig 6.9), induces intolerable streaking artifacts that interfere with the image quality. In the first case, the gridding algorithm fails to handle highly undersampled data and the interpolation window is not able to recover the Cartesian grids from the sparsely sampled $k$-space. In the second case, a fast and abrupt motion induces phase modulations that cause errors when the data along radial views are interpolated into a rectilinear grid. In both situations, the reconstructed image (the inverse Fourier transform) is distorted by artifacts. More investigations are required to study the influence of individual views on the resulting image and to look for possible corrections or alternative reconstructions [128, 358].

The temporal blurring effect is caused by sharing data which may be inconsistent due to 
the presence of ultra-fast motions as in the heart. Experiments in this thesis have shown that the blurring can be eliminated when fewer views are used in the acquisition, because less data are shared. However, this may lead to more streaking artifacts as it enhances the degree of undersampling. On the other hand, experiments in Section 5.3.2 revealed that sharing only the high frequency components in the $k$-space periphery is inefficient for improving the temporal accuracy. Therefore, more studies are required to solve the inconsistent data sharing during image reconstruction and update, possibly with complementary information offered from the use of multiple coil profiles [358].

A further line of future developments and potential applications emerges from the combination of RT-MRI with means for interactive control of imaging parameters including the position and orientation of the imaging plane, FOV, slice offsets, flip angle, and others that affect the image contrast. This may dramatically improve the efficiency in cardiovascular studies [206, 207] and facilitate RT-MRI in planning, guiding, monitoring and controlling therapeutic interventions during surgery $[359,360]$

The access to T1 contrast in spoiled FLASH versions, as shown for the bright signals from inflowing (unsaturated) blood in cardiac MRI, adds potential for the use of a T1shortening contrast agent in "late enhancement" studies of patients where myocardial tissue with compromised perfusion causes a delayed signal enhancement due to a slower or restricted uptake of the contrast agent after bolus injection. This may help in a functional assessment of the myocardium and distinguish dead tissue from damaged tissue [361].

Furthermore, a radial trajectory with a signal readout that starts directly from the center of the $k$-space will allow for ultra-short echo time (UTE) imaging [362], and may be used to better delineate structures like bone or cartilage tissues with short T2 relaxation times [363]. In functional MRI of human human brain activation the application of RT-MRI is motivated by the desire to quickly capture and characterize perfusion changes in the brain. The sensitivity of the gradient echo employed in the FLASH technique to magnetic field inhomogeneities or tissue susceptibilities is used in fMRI of brain activations, since regional increases of cerebral blood flow associated with task performance result in a decrease of the concentration of paramagnetic deoxyhemoglobin in red blood cells. Therefore, further experiments are needed to investigate whether the sensitivity of radial trajectories to such offresonance effects would have additional influence in measuring the small signal changes in functional brain imaging, even though Section 4.3.2 has shown that with respect to morphological or anatomical imaging, they are effectively corrected.

In summary, the results of this thesis demonstrate that the developed radial FLASH 
technique with interleaved sampling and sliding window reconstruction adequately addresses the fundamental challenges of real-time MRI. Based on the implementation on a conventional 3T MRI system and the experimental evaluation in a variety of settings, the proposed RT-MRI method not only outperforms most of the currently used approaches, but also demonstrates its high potential in a wide range of applications. With continuing improvements in engineering and computer technology (to reduce the computational demand imposed by more sophisticated mathematical algorithms), the image quality of the proposed method will be further strengthened with regard to both spatial accuracy and temporal fidelity. It is expected that radial FLASH RT-MRI will become a routine technique for clinical applications and biomedical research. 
- 144 - 

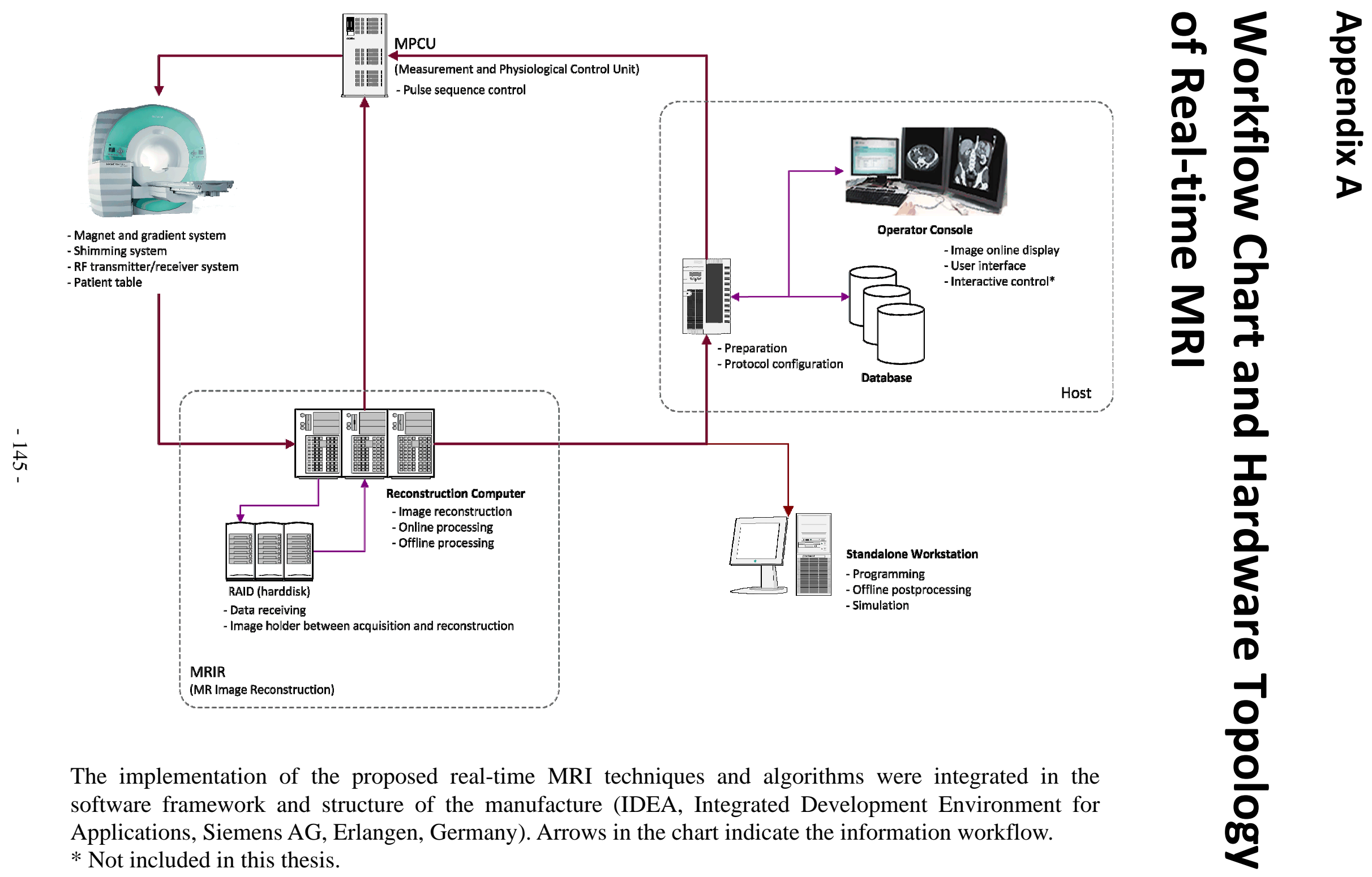

The implementation of the proposed real-time MRI techniques and algorithms were integrated in the software framework and structure of the manufacture (IDEA, Integrated Development Environment for Applications, Siemens AG, Erlangen, Germany). Arrows in the chart indicate the information workflow.

* Not included in this thesis. 


\section{Appendix B}

\section{Quantum Mechanical Description of the NMR Phenomenon}

As described in Chapter 2.1, the spin angular momentum $L$ of any system is characterized by the spin quantum number $I$, also called the nuclear spin or simply spin, which is assigned to each subatomic particle including the entire nucleus:

$$
L=\hbar \cdot \sqrt{I(I+1)}
$$

where $\hbar$ is Planck's constant divided by $2 \pi$ and $I$ is either a non-negative integer or halfinteger $(0,1 / 2,1,3 / 2,2$, etc.), which only depends on the type of particle. The possibility of half-integer quantum numbers is a major difference between spin and orbital angular momentum because the latter can only take integer quantum numbers. The measurable zcomponent of the angular momentum is given by:

$$
L_{z}=\hbar \cdot m_{z}
$$

where $m_{z}$ is a quantum number and may take $(2 I+1)$ values from $-I$ to $+I$. As can be seen here, this spin quantum number is directly proportional to the measured particle's angular momentum and thus its ability to undergo NMR. Its relation with the magnetic moment $\mu_{z}$, which arises from it as a result of the nuclear charge, can be described as:

$$
\mu_{z}=\gamma L_{z}=\gamma \hbar m_{z}
$$

where $\gamma$ is the gyromagnetic ratio - a constant specific to a particular nucleus (approximately 2.674.10 $\mathrm{rad} / \mathrm{Ts}$ for protons) - which depends on the sign, size, and distribution of charge within the material. A random alignment of the individual magnetic moments results in zero total magnetic moment or bulk magnetization.

Nuclei with an even number of both protons and neutrons such as helium $\left({ }^{4} \mathrm{He}\right)$ and carbon $\left({ }^{12} \mathrm{C}\right)$ have $I=0$ and therefore possess no net angular momentum and cannot experience NMR under any conditions; nuclei with odd numbers of both protons and neutrons have spin quantum numbers $I$ that are positive integers, such as nitrogen $\left({ }^{14} \mathrm{~N}, I=1\right)$ and deuterium $\left({ }^{2} \mathrm{H}\right.$, $I=1)$; other nuclei have spins that are half integral including hydrogen $\left({ }^{1} \mathrm{H}, I=1 / 2\right)$, 
fluorine $\left({ }^{19} \mathrm{~F}, I=1 / 2\right)$, sodium $\left({ }^{23} \mathrm{Na}, I=3 / 2\right)$, phosphorus $\left({ }^{31} \mathrm{P}, I=1 / 2\right)$, among which hydrogen nuclei (commonly referred to as protons) are most suitable for MRI due to their sensitivity and biological ubiquity.

When placed in a constant magnetic field $B_{0}$, the magnetic moments will polarize and the energy of interaction of a nucleus depends on the measurable component of the magnetic moments $\mu_{z}$ along the magnetic field:

$$
E=-\mu_{z} B_{0}=\gamma \hbar m_{z} B_{0}
$$

where $B_{0}$ is taken to be along $z$ direction. As can be seen here, the direction of the precession axis $\pm z$ and thus the sign of the spin angular momentum $S$, which describes the energy state occupied by a spin, is determined by the spin $I$, given as $\pm 1 / 2$, for the hydrogen proton, i.e. $m=\left\{-\frac{1}{2},+\frac{1}{2}\right\}$ (spins align with / against the field). Therefore, the potential energy states are:

$$
E_{z}=\left\{-\gamma \hbar \frac{B_{0}}{2},+\gamma \hbar \frac{B_{0}}{2}\right\}
$$

and the energy between states is then:

$$
\Delta E=\gamma \hbar B_{0}
$$

Transitions between the different energy states can be induced by the application of an oscillating magnetic field. Quantum mechanical selection rules dictate that transitions can only take place between adjacent energy levels, hence there is a single energy of transition. Because the energy of radiation of frequency $\omega$ is:

$$
E=\hbar \omega
$$

the frequency of oscillating magnetic field, i.e. Larmor frequency $\omega_{0}$, required to excite the spins is given by:

$$
\omega_{0}=\gamma B_{0}
$$

This explains the reason why the NMR resonance phenomenon occurs only at this discrete frequency. Analysis of the expectation value of the magnetic moment $\mu$ reveals that the zcomponent $\mu_{z}$ remains fixed in the static field, while the transversal component rotates in the xy-plane at angular frequency $\omega_{0}$. Therefore, the vector acts like a precessing gyroscope (or "spinning-top" in most of the classical descriptions). Then if an additional time-varying field with frequency $\omega_{0}$ is created in the xy-plane, the expectation vector $\mu_{z}$ is tilted with respect to the z-axis. In the NMR experiment, this additional field is generated by the RF pulse. Derivation of the expectation vector with respect to time yields:

$$
\frac{d \mu}{d t}=\mu \times \gamma B, \quad B=B_{0}+B_{1}(t)
$$


which obeys the classical equation of motion of a spinning top and holds true for the static and time-dependent magnetic field. It shows that the expectation vector for a single proton can take an arbitrary orientation - regardless of the spin quantization, and it is also valid for the bulk magnetization $M=\sum \mu$ if assuming that the protons do not interact.

For a system (ensemble) of nuclear spins in thermal equilibrium, the population density of each spin state $E_{z}$ is given by the Boltzmann distribution:

$$
P\left(E_{z}\right) \propto e^{-\frac{E_{Z}}{k T}}
$$

where $k$ is the Boltzmann constant and $T$ is temperature. Considering the energy state of hydrogen protons, the relative populations of these states can be calculated as:

$$
\frac{N_{+}}{N_{-}}=\frac{e^{\frac{\gamma \hbar B_{0}}{2 k T}}}{e^{-\frac{\gamma \hbar B_{0}}{2 k T}}}=e^{\frac{\gamma \hbar B_{0}}{k T}}
$$

At room temperature the energy of thermal vibrations is much greater than the separation of the spin energy levels, i.e. $\gamma \hbar B_{0} \ll k T$, which results in near-equal populations in two states. By using power series expansion for an exponential:

$$
\frac{N_{+}}{N_{-}} \sim 1+\frac{\gamma \hbar B_{0}}{k T}
$$

and the fractional difference in population is therefore:

$$
\frac{N_{+}-N_{-}}{N_{-}} \sim \frac{\gamma \hbar B_{0}}{k T}
$$

In a $3 \mathrm{~T}$ magnet at room temperature this population difference is about $\sim 10^{-5}$, a very small amount. It can be increased, based on the equation above, either by reducing the temperature, which is not realistic in clinical use, or by increasing the main static magnetic field $B_{0}$. Because the observed signal is proportional to the net magnetization, i.e. this population difference between these states, it explains here the cause of fundamentally low signal in NMR and also the motivation for higher magnetic fields.

Finally, the aggregate magnetization can be given by averaging over all possible spin energy states:

$$
M_{0}=\rho \gamma \hbar \frac{\sum m_{z} e^{-\frac{E_{Z}}{k T}}}{\sum e^{-\frac{E_{Z}}{k T}}}
$$

where $\rho$ is the number of nuclei per unit volume, i.e. proton density. $M_{0}$ points to the $\mathrm{z}$ direction of $B_{0}$. Considering room temperature and the approximation of the exponential terms 
by $\left(1-E_{m} / k T\right)$, the magnetization for nuclei with spin $I$ can thus be approximated as:

$$
M_{0} \sim \rho \gamma^{2} \hbar^{2}\left[\frac{I(I+1)}{3 k T}\right] B_{0}
$$

For nuclei with $I=1 / 2$ and $m=\left\{-\frac{1}{2},+\frac{1}{2}\right\}$, the magnetization vector is:

$$
M_{0} \sim \rho \frac{\gamma^{2} \hbar^{2}}{4 k T} B_{0}
$$

This relation shows that the magnetization depends primarily on the quantum spin states $I$, the applied magnetic field $B_{0}$, the temperature $T$ of the system, and the distribution $\rho$ of the spins in the volume [364].

In summary, compared to the mechanical model from classical physics, i.e. the "spinning-top" concept, the quantum mechanical model offers a microscopic view of the dynamics of individual nuclear magnetic moments: (1) spin is an intrinsic property of matter, observable only at the atomic level; (2) each particle has a magnetic moment that is directly proportional to both spin and particle composition (gyromagnetic ratio $\gamma$ ); (3) an ensemble of spins can be approximated by a bulk magnetization, which is exclusively exploited in MRI to noninvasively construct tomographic image (refer back to Chapter 2.2). 


\section{Appendix C}

\section{Mathematical Description of the Fourier Transform \& Nyquist Criterion in MRI}

In MRI, there is usually more than one resonance frequency in a sample due to the presence of spatially dependent magnetic field gradients. In this case, the signal resulting from the precessing magnetization is a combination of these frequencies weighted by their respective amplitudes. In order to obtain a spectrum to collect information about the sample, the signal must be de-convolved into its component frequencies. Fourier transform (FT) is the ideal tool to accomplish this task.

First of all, the continuous Fourier Transform $\mathcal{F}(\mathrm{v})$ of a function $\mathfrak{f}(\mathrm{t})$ and its reciprocal, the inverse Fourier transform, can be written as follows:

$$
\begin{aligned}
& \mathcal{F}(v)=\int_{-\infty}^{+\infty} f(t) e^{-i 2 \pi v t} d t \\
& f(t)=\int_{-\infty}^{+\infty} \mathcal{F}(v) e^{i 2 \pi v t} d v
\end{aligned}
$$

Assuming $f(t)$ to represent a signal in time, however, unlike the above equation, the data acquired or sampled in practice is discrete with a limited (finite) duration. Given, for example, a number of $N_{s}$ data points collected at intervals $\Delta t$, by simply considering the Shannon sampling theorem [365], which states that a continuous signal can be properly reconstructed from a discretely sampled function when the sampling rate is at least equal to two times the highest frequency of the signal being sampled, this yields:

$$
\left|v_{\max }\right|=\frac{1}{2 \Delta t}
$$

Then to examine the discrete Fourier transform (DFT) by applying this theorem and replacing $t$ with $N_{s} \Delta t$ :

$$
\mathcal{F}_{D}(v)=\sum_{N_{S}=0}^{M-1} \mathfrak{f}_{D}\left(N_{S} \Delta t\right) e^{\frac{2 \pi i v}{M} N_{S} \Delta t}
$$


it shows that only frequencies, which fall between the range of $\left[-v_{\max }, v_{\max }\right]$, can be uniquely distinguished due to the periodicity of the exponential function, and any frequencies that appear outside of this range will be replicated, or aliased, in this interval. This maximum sampling frequency is often called the Nyquist sampling rate [366], and the sampling law is also known as the Nyquist criterion.

Now considering more specifically MRI, it is already known from Section 2.2.2 that the spin density of the tissue, the 2D Fourier domain data, and the MRI signal are mutually related. The acquired raw data signal directly yields $\mathcal{F}_{D}(v)$, which becomes the starting point from this spatial frequency domain. The discrete sampling of $\mathcal{F}_{D}(v)$, with an interval $\Delta k$, is described mathematically as a multiplication with a Dirac comb or shah function (a periodic train of $\delta$ functions). The Fourier transform, i.e. discrete-frequency FT, of the sampled function, according to its fundamental properties, corresponds to the convolution in the image domain of the transform of the function and the transform of the comb function, with a reciprocal interval width $1 / \Delta k$. This results in the occurrence of periodic object copies, i.e. replication, of $f(t)$ in the image domain, where the distance between the copies is reciprocal to the sample distance $\Delta k$ in $k$-space:

$$
F O V=1 / \Delta k
$$

which yields the same result as Equation 2.16 in Section 2.2.3.

Therefore, in conventional cases where the data are sampled at Cartesian grid points over a rectangular region of $k$-space domain, if the sampling distance in $k$-space and the chosen FOV to be imaged disobey the above rule, so that the Nyquist criterion is violated, the replications of the original object will not be properly distinguished, which then leads to aliasing.

Furthermore, since the $k$-space is only sampled with a finite extent, such "truncation" effect in the spatial frequency domain is mathematically described as the convolution of $\mathcal{F}_{D}(v)$ with a sinc-like function. The Fourier transform into the image domain corresponds to the multiplication of $f(t)$ with a top hat function, which places a limit on the resolution and induces certain blurring. This also explains that the periphery of $k$-space, i.e. high frequency component, contributes to the image details. 


\section{Bibliography}

[1] S. Zhang, K.T. Block, and J. Frahm. Magnetic resonance imaging in real time Advances using radial FLASH. J Magn Reson Imaging, accepted, 2009.

[2] F. Bloch. Nuclear induction. Phys Rev, 70:460-474, 1946.

[3] E.M. Purcell, H.C. Torrey, and R.V. Pound. Resonance absorption by nuclear magnetic moments in a solid. Phys Rev, 69:37-38, 1946.

[4] E.L. Hahn. Nuclear induction due to free larmor precession. Phys Rev, 77:297-298, 1950.

[5] R. Damadian. Tumor detection by nuclear magnetic resonance. Science, 171:1151$1153,1971$.

[6] P.C. Lauterbur. Image formation by induced local interactions: Examples employing nuclear magnetic resonance. Nature, 242:190-191, 1973.

[7] A. Kumar, D. Welti, and R.R. Ernst. NMR fourier zeugmatography. J Magn Reson, 18:69-83, 1975.

[8] S. Ljunggren. A simple graphical representation of fourier-based imaging methods. $J$ Magn Reson, 54:338-343, 1983.

[9] D.B. Twieg. The k-trajectory formulation of the NMR imaging process with applications in analysis and synthesis of imaging methods. Med Phys, 10:610-621, 1983.

[10] P. Mansfield and A.A. Maudsley. Planar and line-scan spin imaging by NMR. In Proc Soc Mag Reson Med, volume XIXth Congress Ampere, pages 247-252, 1976. Heidelberg, Germany.

[11] E.R. Andrew, P.A. Bottomley, W.S. Hinshaw, G.N. Holland, W.S. Moore, and C. Simaroj. NMR images by the multiple sensitive point method: application to larger biological systems. Phys Med Biol, 22:971-974, 1977.

[12] H. Clow and I.R. Young. Britain's brains produce first NMR scans. New Scientist, 80:588, 1978.

[13] R. Damadian. NMR in cancer: Xvi. Fonar image of the live human body. Physiol Chem Phys, 9:97-100, 108, 1977.

[14] P. Mansfield, I.L. Pykett, and P.G. Morris. Coupland re human whole body line-scan imaging by NMR. Brit J Radiol, 51:921-922, 1978.

[15] P. Mansfield. Multi-planar image formation using NMR spin echoes. $J$ Physics $C$ : Solid State Physics, 10:55-58, 1977.

[16] A. Haase, J. Frahm, D. Matthaei, W. Hanicke, and K. Merboldt. FLASH imaging: Rapid NMR imaging using low flip-angle pulses. J Magn Reson, 67:258-266, 1986.

[17] J. Hennig, A. Nauerth, and H. Friedburg. RARE imaging: A fast imaging method for 
clinical MR. Magn Reson Med, 3:823-833, 1986.

[18] D.A. Feinberg, J.D. Hale, J.C. Watts, L. Kaufman, and A. Mark. Halving MR imaging time by conjugation: Demonstration at $3.5 \mathrm{~kg}$. Radiol, 161:527-531, 1986.

[19] S.J. Riederer, T. Tasciyan, F. Farzaneh, J.N. Lee, R.C. Wright, and R.J. Herfkens. MR fluoroscopy: Technical feasibility. Magn Reson Med, 8:1-15, 1988.

[20] R.A. Jones, O. Haraldseth, T.B. Mueller, P.A. Rinck, and A.N. Oksendal. K-space substitution: A novel dynamic imaging technique. Magn Reson Med, 29:830-834, 1993.

[21] J.J. Van Vaals, M.E. Brummer, W.T. Dixon, H.H. Tuithof, H. Engels, R.C. Nelson, B.M. Gerety, J.L. Chezmar, and J.A.D. Boer. Keyhole method for accelerating imaging of contrast agent uptake. J Magn Reson Imaging, 3:671-675, 1993.

[22] M.A. Griswold, P.M. Jakob, R.M. Heidemann, M. Nittka, V. Jellus, J. Wang, B. Kiefer, and A. Haase. Generalized autocalibrating partially parallel acquisitions (GRAPPA). Magn Reson Med, 47:1202-1210, 2002.

[23] M.A. Griswold, P.M. Jakob, M. Nittka, J.W. Goldfarb, and A. Haase. Partially parallel imaging with localized sensitivities (PILS). Magn Reson Med, 44:602-609, 2000.

[24] K.P. Pruessmann, M. Weiger, M.B. Scheidegger, and P. Boesiger. SENSE: Sensitivity encoding for fast MRI. Magn Reson Med, 42:952-962, 1999.

[25] D.K. Sodickson and W.J. Manning. Simultaneous acquisition of spatial harmonics (SMASH): Fast imaging with radiofrequency coil arrays. Magn Reson Med, 38:591-603, 1997.

[26] J. Frahm, A. Haase, and D. Matthaei. Rapid NMR imaging of dynamic processes using the FLASH technique. Magn Reson Med, 3:321-327, 1986.

[27] M.L. Lauzon and B.K. Rutt. Effects of polar sampling in k-space. Magn Reson Med, 36:940-949, 1996.

[28] K. Scheffler and J. Hennig. Reduced circular field-of-view imaging. Magn Reson Med, 40:474-480, 1998.

[29] G.H. Glover and J.M. Pauly. Projection reconstruction techniques for reduction of motion effects in MRI. Magn Reson Med, 28:275-289, 1992.

[30] J.D. O'Sullivan. A fast sinc function gridding algorithm for Fourier inversion in computer tomography. IEEE T Med Imaging, 4:200-207, 1985.

[31] M.A. Bernstein, K.F. King, and X.J. Zhou. Handbook of MRI Pulse Sequences. Elsevier Academic Press, 2004.

[32] E.M. Haacke, R.W. Brown, M.R. Thompson, and Ventatesan R. Magnetic Resonance Imaging: Physical Principles and Sequence Design. Willey-Liss, 1999.

[33] Z.P. Liang and P.C. Lauterbur. Principles of Magnetic Resonance Imaging. IEEE Press Series on Biomedical Engineering, 2000.

[34] F.W. Wehrli, J.R. MacFall, G.H. Glover, N. Grigsby, V. Haughton, and J. Johanson. The dependence of nuclear magnetic resonance (NMR) image contrast on intrinsic and pulse sequence timing parameters. Magn Reson Imaging, 2:3-16, 1984.

[35] T. Villafana. Fundamental physics of magnetic resonance imaging. Radiol Clinics 
North Am, 26:701-715, 1988.

[36] E.L. Hahn. Spin echoes. Phys Rev, 80:580-594, 1950.

[37] J. Frahm, K.-D. Merboldt, W. Hanicke, and A. Haase. Stimulated echo imaging. J Magn Reson, 64:81-93, 1985.

[38] W. Sattin, T.H. Mareci, and K.N. Scott. Exploiting the stimulated echo in nuclear magnetic resonance imaging. I. method. J Magn Reson, 64:177-182, 1985.

[39] D.E. Woessner. Effects of diffusion in nuclear magnetic resonance spin-echo experiments. J Chem Phys, 34:2057, 1961.

[40] S. Hofer and J. Frahm. Topography of the human corpus callosum revisited comprehensive fiber tractography using magnetic resonance diffusion tensor imaging. NeuroImage, 32:989-994, 2006.

[41] K.-D. Merboldt, W. Hanicke, and J. Frahm. Self-diffusion NMR imaging using stimulated echoes. J Magn Reson, 64:479-486, 1985.

[42] U.G. Nolte, J. Finsterbusch, and J. Frahm. Rapid isotropic diffusion mapping without susceptibility artifacts. Whole brain studies using diffusion-weighted single-shot STEAM MR imaging. Magn Reson Med, 44:731-736, 2000.

[43] S. Rieseberg, M. Kuentzel, K.D. Merboldt, and J. Frahm. Diffusion tensor imaging using partial Fourier single-shot stimulated echo acquisition mode magnetic resonance imaging with projection onto convex subsets reconstruction. Magn Reson Med, 54:486-490, 2005.

[44] J.E. Tanner. Use of the stimulated echo in NMR diffusion studies. J Chem Phys, 52:2523, 1970.

[45] J. Frahm, W. Haenicke, and K.-D. Meroldt. Transverse coherence in rapid FLASH NMR imaging. J Magn Reson, 72:307-314, 1987.

[46] J. Frahm and F. Hanefeld. Single voxel proton MR spectroscopy. methods and applications in neuropediatrics. Rivista di Neuroradiologia, 13:7-15, 2000.

[47] B. Wilken, P. Dechent, J. Herms, C. Maxton, E. Markakis, F.A. Hanefeld, and J. Frahm. Quantitative proton magnetic resonance spectroscopy of focal brain lesions. Pediatr Neurol, 23:22-31, 2000.

[48] A. Abragarm. The principles of nuclear magnetism. Oxford, England: Clarendon Press, 1961.

[49] E.L. Hahn. Detection of sea-water motion by nuclear precession. J Geophys Res, 65:776-777, 1960.

[50] W.A. Edelstein, J.M.S. Hutchison, G. Johnson, and T. Redpath. Spin warp NMR imaging and applications to human whole-body imaging. Phys Med Biol, 25:751-756, 1980.

[51] J.K. Mai, G. Paxinos, and T. Voss. Atlas of the human brain. Elsevier Academic Press, 3rd edition, 2008.

[52] J. Frahm. Toward rapid NMR imaging. Encyclopedia of Nuclear Magnetic Resonance. Historical Perspectives., volume 1. Willey, 1996.

[53] R.E. Hendrick, J.B. Kneeland, and D.D. Stark. Maximizing signal-to-noise and 
contrast-to-noise ratios in FLASH imaging. Magn Reson Imaging, 5:117-127, 1987.

[54] W.T. Dixon. Simple proton spectroscopic imaging. Radiol, 153:189, 1984.

[55] H.Y. Carr. Steady-state free precession in nuclear magnetic resonace. Phys Rev, 112:1693, 1958.

[56] C. Westbrook, C.K. Roth, and J. Talbot. MRI in Practice. Blackwell Publishing, 3rd edition, 2005.

[57] J. Frahm and W. Haenicke. Rapid Scan Techniques. Magnetic resonance imaging, volume 1. Mosby, 3 edition, 1999.

[58] A.P. Crawley, M.L. Wood, and R.M. Henkelm. Elimination of transverse coherences in FLASH MRI. Magn Reson Imaging, 8:248-260, 1988.

[59] A. Haase. Snapshot FLASH MRI. Applications to T1, T2 and chemical-shift imaging. Magn Reson Med, 13:77-89, 1990.

[60] M.L. Gyngell, G.L. Nayler, N. Palmer, and M Paley. A comparison of fast acquisition modes in MRI. Magn Reson Imaging, 4:101, 1986.

[61] M.L. Gyngell. The application of steady-state free precession in rapid 2DFT NMR imaging: Fast and CE-FAST sequences. Magn Reson Imaging, 6:415, 1988.

[62] G.H. Glover and N.J. Pelc. A rapid-gated cine MRI technique. Magn Reson Annual, pages 299-333, 1988.

[63] A. Oppelt, R. Graumann, H. Barfuss, H. Fischer, W. Hartl, and W. Schajor. FISP: a new fast MRI sequence. Electromedica, 54:15-18, 1986.

[64] J.L. Duerk, J.S. Lewin, M. Wendt, and C. Petersilge. Remember true FISP? A high SNR, near 1-second imaging method for T2-like contrast in interventional MRI at $.2 \mathrm{~T}$. $J$ Magn Reson Imaging, 8:203-208, 1998.

[65] K. Scheffler. A pictorial description of steady-states in rapid magnetic resonance imaging. Concept Magnetic Res, 11:291-304, 1999.

[66] K. Scheffler and J. Hennig. Is TrueFISP a gradient-echo or a spin-echo sequence? Magn Reson Med, 49:395-397, 2003.

[67] K. Scheffler and S. Lehnhardt. Principles and applications of balanced ssfp techniques. Eur Radiol, 13:2409-2418, 2003.

[68] J. Barkhausen, S.G. Ruehm, M. Goyen, T. Buck, G. Laub, and J.F. Debatin. MR evaluation of ventricular function: true fast imaging with steady-state precession versus fast low-angle shot cine MR imaging: feasibility study. Radiol, 219:264-269, 2001.

[69] V.S. Deshpande, S.M. Shea, G. Laub, O.P. Simonetti, J.P. Finn, and D. Li. 3D magnetization-prepared True-FISP: A new technique for imaging coronary arteries. Magn Reson Med, 46:494-502, 2001.

[70] A. Huber, K. Bauner, B.J. Wintersperger, S.B. Reeder, F. Stadie, E. Mueller, M. Schmidt, E. Winnik, M.F. Reiser, and S.O. Schoenberg. Phase-sensitive inversion recovery (PSIR) single-shot TrueFISP for assessment of myocardial infarction at 3 tesla. Invest Radiol, 41:148-153, 2006.

[71] H.Y. Carr and E.M. Purcell. Effects of diffusion on free precession in nuclear magnetic 
resonance experiments. Phys Rev, 94:630-638, 1954.

[72] S. Meiboom and D. Gill. Modified spin-echo method for measuring nuclear relaxation times. Rev Sci Instruments, 29:688-691, 1958.

[73] Young I.R. Bydder G.lM. MR imaging: clinical use of the inversion recovery sequence. J Comput Assist Tomogr, 9:659-675, 1985.

[74] R. Golfieri, H. Baddeley, J.S. Pringle, and R. Souhami. The role of the STIR sequence in magnetic resonance imaging examination of bone tumours. Brit J Radiol, 63:251-256, 1990.

[75] B. De Coene, J.V. Hajnal, P. Gatehouse, D.B. Longmore, S.J. White, A. Oatridge, J.M. Pennock, I.R. Young, and Bydder G.M. MR of the brain using fluid-attenuated inversion recovery (FLAIR) pulse sequences. Am J Neuroradiol, 13:1555-1564, 1992.

[76] G. McGibney, M.R. Smith, S.T. Nichols, and A. Crawley. Quantitative evaluation of several partial Fourier reconstruction algorithms used in MRI. Magn Reson Med, 30:51-59, 1993.

[77] P. Margosian, F. Schmitt, and D. Purdy. Faster MR imaging: imaging with half the data. Healthcare instrumentation, pages 195-197, 1986.

[78] D.G. Norris. Ultrafast low-angle RARE: U-FLARE. Magn Reson Med, 17:539-542, 1991.

[79] D.G. Norris, P. Bornert, T. Reese, and D. Leibfritz. On the application of ultrafast RARE experiments. Magn Reson Med, 27:142-164, 1992.

[80] J. Frahm, A. Haase, D. Matthaei, K.-D. Merboldt, and W. Haenicke. Rapid NMR imaging using stimulated echoes. J Magn Reson, 65:130-135, 1985.

[81] J. Frahm, W. Haenicke, H. Bruhn, M.L. Gyngell, and K.-D. Merboldt. High-speed STEAM MRI of the human heart. Magn Reson Med, 22:133-142, 1991.

[82] A. Haase, J. Frahm, D. Matthaei, W. Haenicke, H. Bomsdorf, D. Kunz, and R. Tischler. MR imaging using stimulated echoes (STEAM). Radiol, 160:787-790, 1986.

[83] A. Haase and J. Frahm. NMR imaging of spin-lattice relaxation using stimulated echoes. J Magn Reson, 65:481-490, 1985.

[84] J. Finsterbusch and J. Frahm. Half-Fourier single-shot STEAM MRI. Magn Reson Med, 47:611-615, 2002.

[85] A. Karaus, K.-D. Merboldt, J. Graessner, and J. Frahm. Black-blood imaging of the human heart using rapid stimulated echo acquisition mode (STEAM) MRI. J Magn Reson Imaging, 26:1666-1671, 2007.

[86] P. Mansfield and I. Pykett. Biological and medical imaging by NMR. J Magn Reson, 29:355-373, 1978.

[87] I.L. Pykett and R.R. Rzedzian. Instant images of the body by magnetic resonance. Magn Reson Med, 5:563, 1987.

[88] P. Ordidge, R. Coxon, A. Howesman, B. Chapman, R. Turner, M. Stehling, and P. Mansfield. Snapshot head imaging at 0.5T using the echo planar technique. Magn Reson Med, 8:110, 1988. 
[89] P. Ordidge, A. Howesman, R. Coxon, R. Turner, B. Chapman, P. Glover, M. Stehling, and P. Mansfield. Snapshot imaging at $0.5 \mathrm{~T}$ using echo planar techniques. Magn Reson Med, 10:227-240, 1989.

[90] J.G. Ojemann, E. Akbudak, A.Z. Snyder, R.C. McKinstry, M.E. Raichle, and T.E. Conturo. Anatomic localization and quantitative analysis of gradient refocused echo-planar fMRI susceptibility artifact. NeuroImage, 6:156-167, 1997.

[91] C.P. Davis, G.C. McKinnon, L.E. Debatin, and G.K. von Schulthess. Ultra-high-speed MR imaging. Eur Radiol, 6:297-311, 1996.

[92] K.Oshio and D.A. Feinberg. GRASE (gradient- and spin-echo) imaging: A novel fast MRI technique. Magn Reson Med, 10:344-349, 1991.

[93] K.Oshio and D.A. Feinberg. GRASE (gradient- and spin-echo) MR imaging: A new fast clinical imaging technique. Radiol, 181:597-602, 1991.

[94] K.Oshio and D.A. Feinberg. Single-shot GRASE imaging without fast gradients. Magn Reson Med, 26:355-360, 1992.

[95] D.A. Feinberg, B. Kiefer, and G. Johnson. GRASE improves spatial resolution in single shot imaging. Magn Reson Med, 33:529-533, 1995.

[96] W. Umek, A. Ba-Ssalamah, R.Prokesch, R. Mallek, K. Heimberger, and K. Hittmair. Imaging of the brain using the fast-spin-echo and gradient-spin-echo techniques. Eur Radiol, 8:409-415, 1998.

[97] A. Ba-Ssalamah, S. Schick, K. Heimberger, K.F. Linnau, N. Schibany, R. Prokesch, and S. Trattnig. Utrafast magnetic resonance imaging of the brain. Magn Reson Med, 18:237243, 2000.

[98] A. Haase, D. Matthaei, R. Bartkowski, E. Duhmke, and D. Liebfritz. Inversion recovery snapshot FLASH MR imaging. J Comput Assist Tomogr, 13:1036-1040, 1989.

[99] D. Chien, D.J. Atkinson, and R.R. Edelman. Strategies to improve contrast in turboFLASH imaging: reordered phase encoding and k-space segmentation. J Magn Reson Imaging, 1:63-70, 1991.

[100] J. Frahm, K.-D. Merboldt, M.L. Gyngell, W. Hanicke, and D. Chien. 0.3-second FLASH MRI of the human heart. Magn Reson Med, 13:150-157, 1990.

[101] D.J. Atkinson and R.R. Edelman. Cineangiography of the heart in a single breath hold with a segmented turboFLASH sequence. Radiol, 178:357-360, 1991.

[102] M. Brant-Zawadzki, G.D. Gillan, and W.R. Nitz. MP-RAGE: a three-dimensional, T1weighted, gradient-echo sequence initial experience in the brain. Radiol, 182:769-775, 1992.

[103] E.E. de Lange, S.R. Urbanski, J.P. Mugler III, and J.R. Brookeman. Magnetizationprepared rapid gradient echo (MP-RAGE) magnetic resonance imaging of morgagni's hernia. Eur J Radiol, 11:196-199, 1990.

[104] J.P. Mugler III and J.R. Brookema. Three-dimensional magnetization-prepared rapid gradient-echo imaging (3D MP-RAGE). Magn Reson Med, 15:152-157, 1990.

[105] J. Hennig and M. Meuri. Fast imaging using BURST excitation pulses. In Proc Soc Magn Reson Med, 7:238, 1988. 
[106] J. Hennig and M. Hodapp. BURST imaging. Magma Magn Reson Mater Phys Biol Med, 1:39-48, 1993.

[107] P. Le Roux, J. Pauly, and A. Macovski. BURST excitation pulses. In Proc Soc Magn Reson Med, 10:269, 1991.

[108] G.A. Morris and R. Freeman. Selective excitation in Fourier transform nuclear magnetic resonance. J Magn Reson, 29:433, 1978.

[109] I.J. Lowe and R.E. Wysong. Dante ultrafast imaging sequence (DUFIS). J Magn Reson, B101:106-109, 1993.

[110] L. Zha, R..E Wysong, and I.J. Lowe. Optimized ultrafast imaging sequence (OUFIS). In Proc Soc Mag Reson Med, 10:471, 1993.

[111] A. Macovski. Noise in MRI. Magn Reson Med, 36:494-497, 1996.

[112] C.M. Tsai and D.G. Nishimura. Reduced aliasing artifacts using variable-density kspace sampling trajectories. Magn Reson Med, 43:452-458, 2000.

[113] J.F. Schenck, O.M. Mueller, and et al. Souza, S.P. Iron-dependent contrast in NMR imaging of the human brain at 4.0 tesla. In Proc Soc Magn Reson Med, 8:9, 1989.

[114] F.W. Wehrli. Fast-scan magnetic resonance: Principles and applications. Magn Reson $Q, 6: 165-236,1990$.

[115] F.W. Wehrli, T.G. Perkins, A. Shimakawa, and F. Roberts. Chemical shift induced amplitude modulations in images obtained with gradient refocusing. Magn Reson Imaging, 5:157-158, 1987.

[116] M.J. Sanz and E.M. Haacke. Extraction of noise spike in MR imaging. J Magn Reson Imaging, 2:91, 1992.

[117] T.K.F. Foo, N.S. Grigsby, J.D. Mitchell, and B.E. Slayman. SNORE: Spike noise removal and detection. IEEE T Med Imaging, 13:133, 1994.

[118] M. Brown, C. Gustafson, R. Ray, and R.E. Hendrick. MRI: Principles and Artifacts. New York: Raven, 1993.

[119] L. Watson, J. P. Mugler, L. Morris, and J. R. Brookeman. Investigation into the effects of static electrical discharge on MR images. Radiol, 161:511, 1986.

[120] R.M. Henkelman and M.J. Bronskill. Artifacts in magnetic resonance imaging. Rev Magn Reson Med, 2:26-27, 32-34, 77-84, 1987.

[121] R.M. Henkelman. Image artifacts. Magnetic Resonance Imaging. Mosby-Year Book, 1992.

[122] T.A. Spraggins. Spike removal from MR raw data sets. In Proc Intl Soc Mag Reson Med, 8:789, 1987.

[123] C.R. Crawford, D.S. Small, D.G. DallaPiazza, M.G. Eash, and R. S. Stormont. Transient noise suppression using common mode detection and receiver blanking. In Proc Intl Soc Mag Reson Med, 3:939, 1995. Nice, France.

[124] J.W. Goldfarb and F. Schmitt. A robust method to remove spike artifacts through HPFpost-processing. In Proc Intl Soc Magn Reson Med, 5:1646, 1996. New York, USA. 
[125] Y.-H. Kao and J.R. MacFall. Restoration of corrupted k-space raw data for removing striation artifacts on images. In Proc Soc Magn Reson Med, 3:760, 1995. Nice, France.

[126] M. Staemmler and K. Gersonde. Echo shape analysis and image size adjustment on the level of echoes: Improvement of parameter-selective proton images. Magn Reson Med, 3:418-424, 1986.

[127] Y.-H. Kao and J.R. MacFall. Correction of mr k-space data corrupted by spike noise. IEEE T Med Imaging, 19:671, 2000.

[128] K. T. Block. Advanced Methods for Radial Data Sampling in Magnetic Resonance Imaging. PhD thesis, University Goettingen, 2008.

[129] E.M. Haacke and J.A. Tkach. Fast MR imaging: Techniques and clinical applications. Am J Roentgenol, 155:951-964, 1990.

[130] M. Cohen and R. Weisskoff. Ultra-fast imaging. Magn Reson Imaging, 9:1-37, 1991.

[131] M. Cohen. Rapid MR Imaging: techniques and performance characteristics. Radiology. Lippincott. New York, 1992.

[132] J. Petersein and S. Saini. Fast MR imaging: Technical strategies. Am J Roentgenol, 165:1105-1109, 1995.

[133] M. Reiser and S.C. Faber. Recent and future advances in high-speed imaging. Eur Radiol, 7(Suppl. 5):166-173, 1997.

[134] J.G. Pipe. Reconstructing MR images from undersampled data: data-weighting considerations. Magn Reson Med, 43:867-875, 2000.

[135] S. Winkelmann. The exploration of radial trajectories in real-time MRI. PhD thesis, University Karlsruhe, Germany, 2006.

[136] T.P. Trouard, Y. Sabharwal, M.I. Altbach, and A.F. Gmitro. Analysis and comparison of motion-correction techniques in diffusion-weighted imaging. J Magn Reson Imaging, 6:925-935, 1996.

[137] P.M. Bansmann, A.N. Priest, K. Muellerleile, A. Stork, G.K. Lund, M.G. Kaul, and G. Adam. MRI of the coronary vessel wall at $3 \mathrm{~T}$ : Comparison of radial and Cartesian k-space sampling. Am J Roentgenol, 188:70-74, 2007.

[138] D.G. Nishimura, P. Irarrazabal, and C.H. Meyer. A velocity k-space analysis of flow effects in echo-planar and spiral imaging. Magn Reson Med, 33:549-556, 1995.

[139] D.G. Kruger, G.S. Slavin, R. Muthupillai, R.C. Grimm, and S.J. Riederer. An orthogonal correlation algorithm for ghost reduction in MRI. Magn Reson Med, 38:678-686, 1997.

[140] D.C. Noll, J.D. Cohen, C.H. Meyer, and W. Schneider. Spiral k-space MR imaging of cortical activation. J Magn Reson Imaging, 5:49-56, 1995.

[141] C.B. Ahn, J.H. Kim, and Z.H. Cho. High-speed spiral-scan echo planar NMR imaging. IEEE T Med Imaging, 5:2-7, 1986.

[142] E. Yudilevich and H. Stark. Spiral sampling: theory and application to magnetic resonance imaging. J Optical So America, 5:542-553, 1988.

[143] C.H. Meyer, B.S. Hu, D.G. Nishimura, and A. Macovski. Fast spiral coronary artery 
imaging. Magn Reson Med, 28:202-213, 1992.

[144] K.T. Block. Spiralfoermige Abtastung des k-Raumes bei der MagnetresonanzTomographie. Diplomarbeit, University Goettingen, Germany, 2004.

[145] K.T. Block and J. Frahm. Spiral imaging: A critical appraisal. J Magn Reson Imaging, 21:657-668, 2005.

[146] R. Van Tyen and D. Saloner. In Proc Intl Soc Mag Reson Med, 6, 1998.

[147] M. Katoh, E. Spuentrup, A. Buecker, W.J. Manning, R.W. Guenther, and R.M. Botnar. $\mathrm{Mr}$ coronary vessel wall imaging: Comparison between radial and spiral k-space sampling. $J$ Magn Reson Imaging, 23:757-762, 2006.

[148] K.P.N. Forbes, J.G. Pipe, C.R. Bird, and J.E. Heiserman. Propeller MRI: Clinical testing of a novel technique for quantification and compensation of head motion. $J$ Magn Reson Imaging, 14:215-222, 2001.

[149] J.G. Pipe. Motion correction with PROPELLER MRI: Application to head motion and free-breathing cardiac imaging. Magn Reson Med, 42:963-969, 1999.

[150] J.G. Pipe and N. Zwart. Turboprop: Improved PROPELLER imaging. Magn Reson Med, 55:380-385, 2006.

[151] B.J. Wintersperger, V.M. Runge, J. Biswas, C.B. Nelson, A. Stemmer, A.B. Simonetta, M.F. Reiser, L.G. Naul, and S.O. Schoenberg. Brain magnetic resonance imaging at 3 tesla using blade compared with standard rectilinear data sampling. Invest Radiol, 41:586-592, 2006.

[152] S. Skare, R.D. Newbould, D.B. Clayton, and R. Bammer. PROPELLER EPI in the other direction. Magn Reson Med, 55:1298-1307, 2006.

[153] J.G. Pipe, V.G. Farthing, and K.P. Forbes. Multishot diffusion-weighted FSE using PROPELLER MRI. Magn Reson Med, 47:42-52, 2002.

[154] T. Chuang, T. Huang, F. Lin, F. Wang, C. Juan, H. Chung, C. Chen, and K.K. Kwong. PROPELLER-EPI with parallel imaging using a circularly symmetric phased-array RF coil at 3.0 T: Application to high-resolution diffusion tensor imaging. Magn Reson Med, 56:1352$1358,2006$.

[155] S. Naganawa, H. Satake, S. Iwano, H. Kawai, S. Kubota, T. Komada, M. Kawamura, Y. Sakurai, and H. Fukatsu. Contrast-enhanced MR imaging of the brain using T1-weighted FLAIR with blade compared with a conventional spin-echo sequence. Eur Radiol, 18:337342, 2008.

[156] Y. Hirokawa, H. Isoda, Y.S. Maetani, S. Arizono, K. Shimada, and K. Togashi. MRI artifact reduction and quality improvement in the upper abdomen with PROPELLER and prospective acquisition correction (PACE) technique. Am J Roentgenol, 191:1154-1158, 2008.

[157] R.R. Edelman, P. Wielopolski, and F. Schmitt. Echo-planar MR imaging. Radiol, 192:600-612, 1994.

[158] H. Zeng and R.T. Constable. Image distortion correction in EPI: Comparison of field mapping with point spread function mapping. Magn Reson Med, 48:137-146, 2002.

[159] H. Moriguchi, M. Wendt, and J.L. Duerk. Applying the uniform resampling (URS) algorithm to a lissajous trajectory: Fast image reconstruction with optimal gridding. Magn 
Reson Med, 44:766-781, 2000.

[160] R.S. Likes. Moving gradient zeugmatography, 1981.

[161] C.J. Hardy, H.E. Cline, and P.A. Bottomley. Correcting for nonuniform k-space sampling in two dimensional NMR selective excitation. J Magn Reson, 87:639-645, 1990.

[162] Y. Ogura and K. Sekihara. A new method for static imaging of a rotating object. $J$ Magn Reson, 83:177-182, 1989.

[163] X. H. Zhou, Z. P. Liang, S. L. Gewalt, G. P. Cofer, P. C. Lauterbur, and G. A. Johnson. Fast spin-echo technique with circular sampling. Magn Reson Med, 39:23-27, 1998.

[164] L. Axel, G.T. Herman, D.A. Roberts, and L. Dougherty. Linogram reconstruction for magnetic resonance imaging (MRI). IEEE T Med Imaging, 9:1990, 1990.

[165] N. Gai and L. Axel. Elimination of nyquist ghosts in MRI by using fast linogram imaging. J Magn Reson Imaging, 7:1166-1169, 1997.

[166] J.I. Jackson, D.G. Nishimura, and A. Macovski. Twisting radial lines with application to robust magnetic resonance imaging of irregular flow. Magn Reson Med, 25:128-139, 1992.

[167] H.T. Nielsen, E.W. Olcott, and D.G. Nishimura. Improved 2D time-of-fight angiography using a radial-line k-space acquisition. Magn Reson Med, 37:285-291, 1997.

[168] K. Scheffler and J.Hennig. Frequency resolved single-shot MR imaging using stochastic k-space trajectories. Magn Reson Med, 35:569-576, 1996.

[169] D.C. Noll. Multishot rosette trajectories for spectrally selective mr imaging. IEEE T Med Imaging, 16:372-377, 1997.

[170] J.G. Pipe. An optimized center-out k-space trajectory for multishot MRI: Comparison with spiral and projection reconstruction. Magn Reson Med, 42:714-720, 1999.

[171] G.E. Sarty. Single trajectory radial (STAR) imaging. Magn Reson Med, 51:445-451, 2004.

[172] L. Li. Magnetic susceptibility quantification for arbitrarily shaped objects in inhomogeneous fields. Magn Reson Med, 46:907-916, 2001.

[173] J.R. Reichenbach, R. Venkatesan, D.A. Yablonskiy, M.R. Thompson, S. Lai, and E.M. Haacke. Theory and application of static field inhomogeneity effects in gradient-echo imaging. J Magn Reson Imaging, 7:266-279, 1997.

[174] M.T. Alley, G.H. Glover, and N.J. Pelc. Gradient characterization using a Fouriertransform technique. Magn Reson Med, 39:581-587, 1998.

[175] Y. Jung, Y. Jashnani, R. Kijowski, and W.F. Block. Consistent non-Cartesian off-axis MRI quality: Calibrating and removing multiple sources of demodulation phase errors. Magn Reson Med, 57:206-212, 2007.

[176] P. Speier and F. Trautwein. A calibration for radial imaging with large inplane shifts. In Proc Intl Soc Mag Reson Med, 13:2295, 2005.

[177] J.H. Duyn, Y. Yang, J.A. Frank, and J.W. van der Veen. Simple correction method for k-space trajectory deviations in MRI. J Magn Reson, 132:150-153, 1998.

[178] B.M. Dale and J.L. Duerk. The use of measured k-space trajectory for reconstruction 
of radial MRI data. In Proc Intl Soc Mag Reson Med, 10:2334, 2002.

[179] O. Wieben, E. Brodsky, C.A. Mistretta, and W.F. Block. Correction of trajectory errors in radial acquisitions. In Proc Intl Soc Mag Reson Med, 11:298, 2003.

[180] D.C. Peters, J.A. Derbyshire, and E.R. McVeigh. Centering the projection reconstruction trajectory: Reducing gradient delay errors. Magn Reson Med, 50:1-6, 2003.

[181] P. Speier and F. Trautwein. Robust radial imaging with predetermined isotropic gradient delay correction. In Proc Intl Soc Mag Reson Med, 14:2379, 2006.

[182] V. Rasche, D. Holz, and W. Schepper. Radial turbo spin echo imaging. Magn Reson Med, 32:629-638, 1994.

[183] V. Rasche, R.W. Boer, D. Holz, and R. Proksa. Continuous radial data acquisition for dynamic MRI. Magn Reson Med, 34:754-761, 1995.

[184] V. Rasche, D. Holz, and R. Proska. MR fluoroscopy using projection reconstruction multi.gradient-echo (prmGE) MRI. Magn Reson Med, 42:324-334, 1999.

[185] A. Shankaranarayanan, M. Wendt, A.J. Aschoff, J.S. Lewin, and J.L. Duerk. Radial keyhole sequences for low field projection reconstruction interventional MRI. J Magn Reson Imaging, 13:142-151, 2001.

[186] M.I. Altbach, E.K. Outwater, T.P. Trouard, E.A. Krupinski, R.J. Theilmann, A.T. Stopeck, and M. Kono. Radial fast spin-echo method for T1-weighted imaging and T2 mapping of the liver. J Magn Reson Imaging, 16:179-189, 2002.

[187] R.J. Theilmann, A.F. Gmitro, M.I. Altbach, and T.P. Trouard. View-ordering in radial fast spin-echo imaging. Magn Reson Med, 51:768-774, 2004.

[188] H.K. Song, L Dougherty, and M.D. Schnall. Simultaneous acquisition of multiple resolution images for dynamic contrast enhanced imaging of the breast. Magn Reson Med, 46:503-509, 2001.

[189] Y. Cremillieux, A. Briguet, and A. Deguin. Projection-reconstruction methods: Fast imaging sequences and data processing. Magn Reson Med, 32:23-32, 1994.

[190] D.C. Peters, F.R. Korosec, T.M. Grist, W.F. Block, J.E. Holden, K.K. Vigen, and C.A. Mistretta. Undersampled projection reconstruction applied to MR angiography. Magn Reson Med, 43:91-101, 2000.

[191] K.K. Vigen, D.C. Peter, T.M. Grist, W.F. Block, and C.A. Mistretta. Undersampled projection-reconstruction imaging for time-resolved contrast enhanced imaging. Magn Reson Med, 43:170-176, 2000.

[192] A.C. Larson and O.P. Simonetti. Real-time cardiac cine imaging with spider: Steadystate projection imaging with dynamic echo-train readout. Magn Reson Med, 46:1059-1066, 2001.

[193] V Rasche, R Proksa, R Sinkus, P Bornert, and H Eggers. Resampling of data between arbitrary grids using convolution interpolation. IEEE T Med Imaging, 18:385-392, 1999.

[194] H. Sedarat and D.G. Nishimura. On the optimality of the gridding reconstruction algorithm. IEEE T Med Imaging, 19:306-317, 2000.

[195] GN Ramachandran and AV Lakshminarayanan. Three-dimensional reconstruction 
from radiographs and electron micrographs: application of convolutions instead of Fourier transforms. In Proc Natl Acad Sci USA, 68:2236-2240, 1971.

[196] P.M. Joseph. Sampling errors in projection reconstruction MRI. Magn Reson Med, 40:460-466, 1998.

[197] J.G. Pipe and P. Menon. Sampling density compensation in MRI: Rationale and an iterative numerical solution. Magn Reson Med, 41:179-186, 1999.

[198] F. Aurenhammer. Voronoi diagrams - a survey of a fundamental geometric data structure. ACM Computing Surveys, 23:345-405, 1991.

[199] J.F. Kaiser. Nonrecursive digital filter design using the $I_{0}$-sinh window function. In Proc IEEE Int Symp on Circuits and Syst, pages 20-23, 1974.

[200] J. Jackson, C.H. Meyer, D.G. Nishimura, and A. Macovski. Selection of a convolution function for Fourier inversion using gridding. IEEE T Med Imaging, 10:473-478, 1991.

[201] P.J. Beatty, D.G. Nishimura, and J.M. Pauly. Rapid gridding reconstruction with a minimal oversampling ratio. IEEE T Med Imaging, 24:799-808, 2005.

[202] F. Farzaneh, S.J. Riederer, J.N. Lee, T. Tasciyan, R.C. Wright, and C.E. Spritzer. MR fluoroscopy: Initial clinical studies. Radiol, 171:545-549, 1989.

[203] R.C. Wright, S.J. Riederer, F. Farzaneh, P.J. Rossman, and Y. Liu. Real-time MR fluoroscopic data acquisition and image reconstruction. Magn Reson Med, 12:407-441, 1989.

[204] A.F. Gmitro, A.R. Ehsani, T.A. Berchem, and R.J. Snell. A real-time reconstruction system for magnetic resonance imaging. Magn Reson Med, 35:734-740, 1996.

[205] J.P. Debbins, S.J. Riederer, P.J. Rossman, R.C. Grimm, J.P. Felmlee, J.F. Breen, and R.L. Ehman. Cardiac magnetic resonance fluoroscopy. Magn Reson Med, 36:588-595, 1996.

[206] A.E. Holsinger, R.C. Wright, S.J. Riederer, R.C. Grimm, and J.K. Maier. Real-time interactive magnetic resonance imaging. Magn Reson Med, 14:541-553, 1990.

[207] C.J. Hardy, R.D. Darrow, E.J. Nieters, P.B. Roemer, R.D. Watkins, W.J. Adams, N.R. Hattes, and J.K. Maier. Real-time acquisition, display, and interactive graphic control of NMR cardiac profiles and images. Magn Reson Med, 29:667-673, 1993.

[208] K. Kose, T. Haishi, A. Caprihan, and E. Fukushima. Real-time NMR imaging systems using personal computers. J Magn Reson, 124:35-41, 1997.

[209] T. Haishi and K. Kose. Real-time image reconstruction and display system for MRI using a high-speed personal computer. J Magn Reson, 134:138-141, 1998.

[210] X. Hu. On the "keyhole" technique (letter to the editor). J Magn Reson Imaging, 4:231, 1994.

[211] F.R. Korosec, R. Frayne, T.M. Grist, and C.A. Mistretta. Time-resolved contrastenhanced 3D MR angiography. Magn Reson Med, 36:345-351, 1996.

[212] J.H. Gao, J. Xiong, S. Lai, E.M. Haacke, M.G. Woldorff, J. Li, and P.T. Fox. Improving the temporal resolution of functional mr imaging using keyhole. Magn Reson Med, 35:854-860, 1996.

[213] J. Xiong, P.T. Fox, and J.H. Gao. The effects of k-space data undersampling and discontinuities in keyhole functional MRI. Magn Reson Imaging, 17:109-119, 1999. 
[214] C. Oesterle, R. Strohschein, M. Koehler, M. Schnell, and J. Hennig. Benefits and pitfalls of keyhole imaging, especially in first-pass perfusion studies. J Magn Reson Imaging, 11:312-323, 2000.

[215] M. Busch, A. Bornstedt, M. Wendt, J.L. Duerk, J.S. Lewin, and D. Groememeyer. Fast "real time" imaging with different k-space update strategies for interventional procedures. $J$ Magn Reson Imaging, 8:944-954, 1998.

[216] J.L. Duerk, J.S. Lewin, and D.H. Wu. Application of keyhole imaging to interventional MRI: A simulation study to predict sequence requirements. J Magn Reson Imaging, 6:918-924, 1996.

[217] Y. Cao and D.N. Levin. Feature-recognizing MRI. Magn Reson Med, 30:305-317, 1993.

[218] H. Moriguchi and J.L. Duerk. Iterative next-neighbor regridding (INNG): improved reconstruction from nonuniformly sampled k-space data using rescaled matrices. Magn Reson Med, 51:343-352, 2004.

[219] R.E. Gabr, P. Aksit, P.A. Bottomley, A.-B.M. Youssef, and Y.M. Kadah. Deconvolution-interpolation gridding (DING): accurate reconstruction for arbitrary k-space trajectories. Magn Reson Med, 56:1182-1191, 2006.

[220] G.E. Sarty, R. Bennett, and R.W. Cox. Direct reconstruction of non-Cartesian k-space data using a nonuniform fast Fourier transform. Magn Reson Med, 45:908-915, 2001.

[221] L. Sha, H. Guo, and A.W. Song. An improved gridding method for spiral MRI using nonuniform fast Fourier transform. J Magn Reson, 162:250-258, 2003.

[222] J.A. Fessler. On nufft-based gridding for non-Cartesian MRI. J Magn Reson, 188:191$195,2007$.

[223] K.T. Block, M. Uecker, and J. Frahm. Undersampled radial MRI with multiple coils. Iterative image reconstruction using a total variation constraint. Magn Reson Med, 57:10861098, 2007.

[224] J.B. Weaver, Y. Xu, D.M. Healy, and J.R. Driscoll. Wavelet-encoded MR imaging. Magn Reson Med, 24:275-287, 1992.

[225] D. Rosenfeld. An optimal and efficient new gridding algorithm using singular value decomposition. Magn Reson Med, 40:14-23, 1998.

[226] D. Rosenfeld. New approach to gridding using regularization and estimation theory. Magn Reson Med, 48:193-202, 2002.

[227] L.P. Panych, P.D. Jakab, and F.A. Jolesz. Implementation of wavelet-encoded MR imaging. J Magn Reson Imaging, 3:649-655, 1993.

[228] W.E. Kyriakos, L.P. Panych, D.F. Kacher, C.F. Westin, S.M. Bao, R.V. Mulkern, and F.A. Jolesz. Sensitivity profiles from an array of coils for encoding and reconstruction in parallel (SPACE RIP). Magn Reson Med, 44:301-308, 2000.

[229] X. Hu and T. Parrish. Reduction of field of view for dynamic imaging. Magn Reson Med, 31:691-694, 1994.

[230] T. Parrish and X. Hu. A hybrid technique for dynamic imaging. Magn Reson Med, 44:51-55, 2000. 
[231] Q.S. Xiang and R.M. Henkelman. Dynamic image reconstruction: MR movies from motion ghosts. J Magn Reson Imaging, 2:679-685, 1992.

[232] Z.P. Liang, H. Jiang, C.P. Hess, and P.C. Lauterbur. Dynamic imaging by model estimation. Int J Imag Syst Technol, 8:551-557, 1997.

[233] Z.P. Liang, T. Constable, E.M. Haacke, F. Boada, M. Smith, and P.C. Lauterbur. Constrained reconstruction methods in MR imaging. Rev Magn Reson Med, 4:67-185, 1992.

[234] B. Madore, G.H. Glover, and N.J. Pelc. Unaliasing by Fourier-encoding the overlaps using the temporal dimension (UNFOLD), applied to cardiac imaging and fMRI. Magn Reson Med, 42:813-828, 1999.

[235] J. Tsao. On the UNFOLD method. Magn Reson Med, 47:202-207, 2002.

[236] P. Kellman, J.M. Sorger, F.H. Epstein, and E.R. McVeigh. Low latency temporal filter design for real-time MRI using UNFOLD. Magn Reson Med, 44:933-939, 2000.

[237] J. Tsao, P. Boesiger, and K.P. Pruessmann. k-t BLAST and k-t SENSE: dynamic MRI with high frame rate exploiting spatiotemporal correlations. Magn Reson Med, 50:1031-1042, 2003.

[238] P. Kellman, F.H. Epstein, and E.R. McVeigh. Adaptive sensitivity encoding incorporating temporal filtering (TSENSE). Magn Reson Med, 45:846-852, 2001.

[239] B. Madore. UNFOLD-SENSE: A parallel MRI method with self-calibration and artifact suppression. Magn Reson Med, 52:310-320, 2004.

[240] F.A. Breuer, P. Kellman, M.A. Griswold, and P.M. Jakob. Dynamic autocalibrated parallel imaging using temporal grappa (TGRAPPA). Magn Reson Med, 53:981-985, 2005.

[241] F. Huang, J. Akao, S. Vijayakumar, G.R. Duensing, and M. Limkeman. k-t grappa: A k-space implementation for dynamic MRI with high reduction factor. Magn Reson Med, 54:1172-1184, 2005.

[242] D. Xu, K.F. King, and Z.P. Liang. Improving k-t SENSE by adaptive regularization. Mag Reson Med, 57:918-930, 2007.

[243] M.S. Hansen, C. Baltes, J. Tsao, S. Kozerke, K.P. Pruessmann, and H. Eggers. k-t BLAST reconstruction from non-Cartesian k-t space sampling. Magn Reson Med, 55:85-91, 2006.

[244] C.A. Mistretta, O. Wieben, J. Velikina, W. Block, J. Perry, Y. Wu, K. Johnson, and Y. Wu. Highly constrained backprojection for time-resolved MRI. Magn Reson Med, 55:3040, 2006.

[245] P.A. Turski, F.R. Korosec, T.J. Carroll, D.S. Willig, T.M. Grist, and C.A. Mistretta. Contrast-enhanced magnetic resonance angiography of the carotid bifurcation using the timeresolved imaging of contrast kinetics (TRICKS) technique. Top Magn Reson Imaging, $12: 175-181,2001$.

[246] C.A. Mistretta. Undersampled radial MR acquisition and highly constrained back projection (HYPR) reconstruction: Potential medical imaging. Applications in the postNyquist era. J Magn Reson Imaging, 29:501-516, 2009.

[247] D. Donoho. Compressed sensing. IEEE T Inform Theory, 52:1289-1306, 2006. 
[248] M. Lustig, D. Donoho, and J.M. Pauly. Sparse MRI: The application of compressed sensing for rapid MR imaging. Magn Reson Med, 58:1182-1195, 2007.

[249] U. Gamper, P. Boesiger, and S. Kozerke. Compressed sensing in dynamic MRI. Magn Reson Med, 59:365-373, 2008.

[250] M. Lustig, D.L. Donoho, J.M. Santos, and J.M. Pauly. Compressed sensing MRI. IEEE Signal Proc Mag, 25:72-82, 2008.

[251] J.A. d'Arcy, D.J. Collins, I.J. Rowland, A.R. Padhani, and M.O. Leach. Applications of sliding window reconstruction with Cartesian sampling for dynamic contrast enhanced MRI. NMR Biomed, 15:174-183, 2002.

[252] M. Zaitsev, K. Zilles, and N.J. Shah. Shared k-space echo planar imaging with keyhole. Mag Reson Med, 45:109-117, 2001.

[253] V. Rasche, D. Holz, J. Koehler, R. Proksa, and P. Roeschmann. Catheter tracking using continuous radial MRI. Magn Reson Med, 37:963-968, 1997.

[254] A. Shankaranarayanan, O.P. Simonetti, G. Laub, J.S. Lewin, and J.L. Duerk. Segmented k-space and real-time cardiac cine MR imaging with radial trajectories. Radiol, 221:827-836, 2001.

[255] H.K. Song and L. Dougherty. k-space weighted image contrast (KWIC) for contrast manipulation in projection reconstruction MRI. Magn Reson Med, 44:825-832, 2000.

[256] H.K. Song and L. Dougherty. Dynamic MRI with projection reconstruction and KWIC processing for simultaneous high spatial and temporal resolution. Magn Reson Med, 52:815824, 2004.

[257] K.S. Nayak, C.H. Cunningham, J.M. Santos, and J.M. Pauly. Real-time cardiac MRI at 3 tesla. Magn Reson Med, 51:655-660, 2004.

[258] J. Hennig, O. Speck, and K. Scheffler. Optimizatin of the signa behavior in the transition to driven equilibrium in steady-state free precession sequences. Magn Reson Med, 48:801-809, 2002.

[259] T. Schaeffter, S. Weiss, and P. Boernert. A SAR-reduced steady-state free precessing (SSFP) acquisition. In Proc Intl Soc Magn Reson Med, page 2351, 2002.

[260] K.F. King, A. Ganin, X.J. Zhou, and M.A. Bernstein. Concomitant gradient field effects in spiral scans. Magn Reson Med, 41:103-112, 1999.

[261] C.T. Sica and C.H. Meyer. Concomitant gradient field effects in balanced steady-state free precession. Magn Reson Med, 57:721-730, 2007.

[262] V.S. Deshpande, S.M. Shea, and D. Li. Artifact reduction in true-FISP imaging of the coronary arteries by adjusting imaging frequency. Magn Reson Med, 49:803-809, 2003.

[263] H-L. Lee, G.M. Pohost, and K.S. Nayak. Gated and real-time wideband SSFP cardiac imaging at 3T. In Proc Intl Soc Mag Reson Med, 14:143, 2006.

[264] R.S. Saleh, D.G. Lohan, K. Nael, M. Grover-McKay, and J.P. Finn. Cardiovascular MRI at 3T. Applied Radiol, pages 10-26, 2007.

[265] F. Huang, S. Vijayakumar, Y. Li, S. Hertel, and G.R. Duensing. A software channel compression technique for faster reconstruction with many channels. Magn Reson Imaging, 
26:133-141, 2008.

[266] M. Buehrer, K.P. Pruessmann, P. Boesiger, and S. Kozerke. Array compression for MRI with large coil arrays. Magn Reson Med, 57:1131-1139, 2007.

[267] J.L. Derner and B.M. Kerman. Comparison of standardized echography with magnetic resonance imaging to measure extraocular muscle size. Am J Ophthalmol, 118:351-361, 1994.

[268] L.T. Bilaniuk, S.W. Atlas, and R.A. Zimmermann. Magnetic resonance imaging of the orbit. Radiol Clin North Am, 25:509-528, 1987.

[269] N. Yasuhiro. MRI for eye movement. Jpn Orthopt J, 28:1-9, 2000.

[270] J.L. Demer, J.M. Miller, E.Y. Koo, and A.L. Rosenbaum. Quantitative magnetic resonance morphometry of extraocular muscles: a new diagnostic tool in paralytic strabismus. J Pediatr Ophthalmol Strabismus, 31:177-188, 1994.

[271] J.M. Miller, J.L. Derner, and A.L. Rosenbaum. Effect of transposition surgery on rectus muscle paths by magnetic resonance imaging. Ophthalmol, 100:475-487, 1993.

[272] C.C. Bailey, J. Kabala, R. Laitt, M. Weston, P. Goddard, H.B. Hoh, M.J. Potts, and R.A. Harrad. Cine magnetic resonance imaging of eye movements. Eye, 7:691-693, 1993.

[273] F.M. Jewell, R.D. Laitt, C.C. Bailey, C.J. Wakeley, M.J. Potts, R.J. Harrad, M.Weston, J.Kabala, and P. Goddard. Video loop MRI of ocular motility disorders. J Computer Assis Tomogr, 19:39-43, 1995.

[274] R. Ghabrial, M.J. Potts, R.A. Harrad, J. Hunter, J. Kabala, and D. Macey. Assessment of the anophthalmic socket with dynamic cine-MRI. Obit, 16:207-216, 1997.

[275] W. Cadera, E. Viirre, and S. Karlik. Cine magnetic resonance imaging of ocular motility. J Pediatr Ophthalmol Strabismus, 29:120-122, 1992.

[276] J.N. Bloom, W. Cadera, E. Heiberg, and S. Karlik. A magnetic resonance imaging study of horizontal rectus muscle palsies. J Pediatr Ophthalmol Strabismus, 30:296-300, 1993.

[277] C. Scheiber, C. Speeg-Schatz, and J. Chambron. Technical note. technique for MRI of ocular motility. J Computer Assis Tomogr, 21:442-446, 1997.

[278] T.Littan, M. Bendszus, E. Hofmann, and W. Lieb. Dynamische MRT-Darstellung von Orbitaerkrankungen und Augenmotilitaetsstoerungen. Ophthalmologe, 98:86-87, 2001.

[279] M.D. Abramoff, Ad P.G. van Gils, G.H. Jansen, and M.P. Mourits. MRI dynamic color mapping: A new quantitative technique for imaging soft tissue motion in the orbit. Invest Ophthalmol Vis Sci, 41:3256-3260, 2000.

[280] L. Jaeger, U. Welge-Luessen, I. Lanzl, and M. Reiser. Imaging of eye movement with fast MRI. J Computer Assis Tomogr, 21:447-451, 1997.

[281] J.-H. Lee, K.-I. Yun, I.-W. Park, H.-M. Choi, and M.-S. Park. Comparison of static MRI and pseudo-dynamic MRI in tempromandibular joint disorder patients. Korean J Oral Maxillofacial RPdd, 36:199-206, 2006.

[282] W.F. Conway, C.W. Hayes, R.L. Campbell, and D.M. Laskin. Temporomandibular joint motion: efficacy of fast low-angle shot MR imaging. Radiol, 172:821-826, 1989.

[283] M. Maeda, S. Itou, Y. Ishii, K.Yamamoto, Y. Kawamura, T. Matsuda, N. Hayashi, and 
Y. Ishii. Temporomandibular joint movement. evaluation of protrusive splint therapy with GRASS MR imaging. Acta Radiologica, 33:410-413, 1992.

[284] Y.-F. Ren, P.L. Westesson, and A. Isberg. Magnetic resonance imaging of the temporomandibular joint: Value of pseudodynamic images. Oral Surg Oral Med Oral Pathol Oral Radiol Endod, 81:110-123, 1996.

[285] K.A. Burnett, C.L. Davis, and J. Read. Dynamic display of the temporomandibular joint meniscus by using "fast-scan" MR imaging. Am J Roentgenol, 149:959-962, 1987.

[286] K.P. Schellhas, H.M. Fritts, K.B. Heithoff, J.A. Jahn, C.H. Wilkes, and M.R. Omlie. Temporomandibular joint: MR fast scanning. Cranio, 6:209-216, 1988.

[287] K.P. Schellhas, C.H. Wilkes, H.M. Fritts, M.A. Omlie, K.B. Heithoff, and J.A. Jahn. Temporomandibular joint: MR imaging of internal derangements and postoperative changes. Am J Roentgenol, 150:381-389, 1988.

[288] W.F. Conway, C.W. Hayes, and R.L. Campbell. Dynamic magnetic resonance imaging of the temporomandibular joint using FLASH sequences. J Oral Maxillofac Surg, 46:930-938, 1988.

[289] J.M. Fulmer and S.E. Harms. The temporomandibular joint. Top Magn Reson Imag, 1:75-84, 1989.

[290] T.J. Vogl, D. Eberhard, C. Bergman, and J. Lissner. Incremental hydraulic jaw opener for MR imaging of the temporomandibular joint. J Magn Reson Imaging, 2:479-482, 1992.

[291] K.A. Bell, K.D. Miller, and J.P. Jones. Cine magnetic resonance imaging of the temporomandibular joint. J Craniomandibular Practice, 10:313-317, 1992.

[292] J.C. Quemar, S. Akoka, H. Romdane, and J.D. de Certaines. Evaluation of a fast pseudo-cinematic method for magnetic resonance imaging of the temporomandibular joint. Dento Maxillo Facial Radiol, 22:61-68, 1993.

[293] M. Behr, P. Held, A. Leibrock, C. Fellner, and G. Handel. Diagnostic potential of pseudo-dynamic MRI (cine mode) for evaluation of internal derangement of the TMJ. Eur $J$ Radiol, 23:212-215, 1996.

[294] D. Eberhard, H.P. Bantleon, and W. Steger. Functional magnetic resonance imaging of temporomandibular joint disorders. Eur J Orthodontics, 22:489-497, 2000.

[295] H. Yoshida, H. Hirohata, K. Onizawa, M. Niitsu, and Y. Itai. Flexure deformation of the temporomandibular joint disk in pseudodynamic magnetic resonance images. Oral Surg Oral Med Oral Pathol Oral Radiol Endod, 89:104-111, 2000.

[296] A. Beer, A. Kolk, A. Neff, T. Treumann, and E.J. Rummeny. Cine-MRT des Kiefergelenks im Vergleich zur konventionellen MRT und Achsiographie. Fortschr Roentgenstr, 176:506-512, 2004.

[297] T.J. Vogl and N. Abolmaali. Magnetresonanztomographie des Temporomandibulargelenkes: Untersuchungstechnik, Ergebnisse, Indikationsstellung. Am J Roentgenol, 173:969979, 2001.

[298] A. Maniere-Ezvan, T. Havet, J.-M. Franconi, J.-C. Quemar, and J.-D. de Certaines. Cinematic study of temporomandibular joint motion using ultrafast magnetic resonance imaging. J Craniomandibular Practice, 17:262-267, 1999. 
[299] Y.-J. Chen, L.M. Gallo, D. Meier, and S Palla. Dynamic magnetic resonance imaging technique for the study of the temporomandibular joint. J Orofacial Pain, 14:65-73, 2000.

[300] N.D. Abolmaali, J. Schmitt, W. Schwarz, D.E. Toll, S. Hinterwimmer, and T.J. Vogl. Visualization of the articular disk of the temporomandibular joint in near-real-time MRI: feasibility study. Eur Radiol, 14:1889-1894, 2004.

[301] E.Y. Wang, T.P. Mulholland, B.K. Pramanik, A.O. Nusbaum, J. Babb, A.G. Pavone, and K.E. Fleisher. Dynamic sagittal half-Fourier acquired single-shot turbo spin-echo MR imaging of the temporomandibular joint: Initial experience and comparison with sagittal oblique proton-attenuation images. Am J Neuroradiol, 28:1126-1132, 2007.

[302] Y. Shimazaki, K. Saito, S. Matsukawa, R. Onizawa, F. Kotake, R. Nishio, and K. Abe. Image quality using dynamic $\mathrm{mr}$ imaging of the temporomandibular joint with True-FISP sequence. Magn Reson Med Sci, 6:15-20, 2007.

[303] A. Bumann and U. Lotzmann. Funktionsdiagnostik und Therapieprinzipien. Farbatlanten der Zahnmedizin. Georg Thieme Verlag Stuttgart, 2000.

[304] P.M. Bonutti, J.F. Norfray, R.J. Friedman, and B.M. Genez. Kinematic MRI of the shoulder. J Comput Assist Tomogr, 17:666-669, 1993.

[305] N. Sans, G. Richardi, J.-J. Railhac, J. Assoun, D. Fourcade, M. Mansat, J. Giron, H. Chianassa, T. Jartaud, and J.-J. Paul. Kinematic mr imaging of the shoulder: Normal patterns. Am J Roentgenol, 167:1517-1522, 1996.

[306] T. Imaeda, R. Nakamura, K. Shionoya, and N. Makino. Ulnar impaction syndrome: MR imaging findings. Radiol, 201:495-412, 1996.

[307] V. Vedi, A. Williams, S.J. Tennant, E. Spouse, D.M. Hunt, and W.M.W. Gedroyc. Meniscal movement. An in-vivo study using dynamic MRI. J Bone Joint Surg [Br], 81:37-41, 1999.

[308] S. Nakagawa, Y. Kadoya, A. Kobayashi, I. Tatsumi, N. Nishida, and Y. Yamano. Kinematics of the patella in deep flexion. Analysis with magnetic resonance imaging. $J$ Bone Joint Surg Am, 85:1238-1242, 2003.

[309] M.A.R. Freeman and V. Pinskerova. The movement of the knee studied by magnetic resonance imaging. Clin Orthop Relat Res, 410:35-43, 2003.

[310] A. Williams. Understanding knee motion - the application of "dynamic MRI". J Bone Joint Surg [Br], 86(Supp 4):471, 2004.

[311] M.C. Logan, A. Williams, J. Lavelle, W. Gedroyc, and M. Freeman. What really happens during the lachman test? A dynamic MRI analysis of tibiofemoral motion. Am J Sports Med, 32:2, 2004.

[312] F.G. Shellock, T.K. Foo, A.L. Deutsch, and J.H. Mink. Patellofemoral joint: evaluation during active flexion with ultrafast spoiled GRASS MR imaging. Radiol, 180:581-585, 1991.

[313] C. Muhle, J. Brossmann, U.H. Melchert, C. Schriider, R. de Boer, R.P. Spielmann, and M. Heller. Functional MRI of the patellofemoral joint: comparison of ultrafast MRI, motiontriggered cine MRI and static MRI. Eur Radiol, 5:371-378, 1995.

[314] C. Muhle, G. Brinkmann, F. Wesner, and M. Heller. Kinematic MR imaging of the ankle: initial results with ultra-fast sequence imaging. Acta Radiol, 38:885-889, 1997. 
[315] C.M. Powers, F.G. Shellock, and M.Pfaff. Quantification of patellar tracking using kinematic MRI. J Magn Reson Imaging, 8:724-732, 1998.

[316] D. Witonski and B. Goraj. Patellar motion analyzed by kinematic and dynamic axial magnetic resonance imaging in patients with anterior knee pain syndrome. Arch Orthop Trauma Surg, 119:46-49, 1999.

[317] H.H. Quick, M.E. Ladd, M. Hoevel, S. Bosk, J.F. Debatin, G. Laub, and T. Schroeder. Real-time MRI of joint movement with TrueFISP. J Magn Reson Imaging, 15:710-715, 2002.

[318] C.E. Draper, J.M. Santos, L.C. Kourtis, T.F. Besier, M.Fredericson, G.S. Beaupre, G.E. Gold, and S.L. Delp. Feasibility of using real-time MRI to measure joint kinematics in 1.5T and open-bore 0.5T systems. J Magn Reson Imaging, 28:158-166, 2008.

[319] F.G. Shellock, J.H. Mink, A. Deutsch, and B.D. Pressman. Kinematic magnetic resonance imaging of the joints: Techniques and clinical application. Magn Reson Quarterly, 7:104-135, 1991.

[320] Functional evaluation of joints using kinematic MRI. Website, October 242000.

[321] G. Fant. Acoustic Theory of Speech Production. Mouton, The Hague, 1960.

[322] J. Koreman. Decoding Linguistic Information in the Glottal Airflow. PhD thesis, Nijmegen University, 1996.

[323] K. Honda, S. Maeda, and M. Sugito. Articulation changes in different voicing patterns. In International Congress of Phonetic Sciences (ICPhS), Saarbruecken, 16:237-240, 2007.

[324] B. Pompino-Marschall. Einfuehrung in die Phonetik. de Gruyter, Berlin, 2nd edition, 2003.

[325] B. J. Kroeger, P. Hoole, R. Sader, C. Geng, B. Pompino-Marschall, and C. Neuschaefer-Rube. MRT-Sequenzen als Datenbasis eines visuellen Artikulationsmodells. Hals Nasen Ohren, 52:837-851, 2004.

[326] J. Roemer. RT-MRI der Sprechbewegungen. Bachelor's thesis, University of Wuerzburg, 2009.

[327] T. Baer, O.C. Gore, L.C. Gracco, and P.W. Nye. Analysis of vocal tract shape and dimensions using magnetic resonance imaging: Vowels. J Acoust Soc Am, 90:799-828, 1991.

[328] K. Mathiak, U. Klose, H. Ackermann, I. Hertrich, W.-E. Kincses, and W. Grodd. Stroboscopic articulography using fast magnetic resonance imaging. Int J Lang Comm Dis, 35:419-425, 2000.

[329] K. Mady, R. Sader, A. Zimmermann, Ph. Hoole, A. Beer, H.F. Zeilhofer, and Ch. Hannig. Use of real-time MRI in assessment of consonant articulation before and after tongue surgery and tongue reconstruction, 2001.

[330] S. Narayanan, K. Nayak, D. Byrd, and S. Lee. An approach to real-time magnetic resonance imaging for speech production. J Acous Soc Am, 115:1771-1776, 2004.

[331] E. Bresch, Y.-C. Kim, K Nayak, D. Byrd, and S. Narayanan. Seeing speech: Capturing vocal tract shaping using real-time magnetic resonance imaging. IEEE Sign Proc Magazine, pages 123-132, 2008.

[332] A.V. Lakshminarayanan, Sungbok Lee, and Martin J. McCutcheon. MR imaging of 
the vocal tract during vowel production. J Magn Reson Imaging, 1:71-76, 1991.

[333] D. Demolin, V. Delvaux, T. Metens, and A. Soquet. Determination of velum opening for French nasal vowels by magnetic resonance imaging. $J$ Voice, 17:454-467, 2002.

[334] S. Masaki, M.K. Tiede, K. Honda, Y. Shimada, I. Fujimoto, Y. Nakamura, and N. Ninomiya. MRI based speech production study using a synchronized sampling method. $J$ Acoust Soc Jpn, 20:377-381, 1999.

[335] A.J. Miller. Deglutition. Physiol Rev, 62:129-184, 1982.

[336] W.J. Dodds. The physiology of swallowing. Dysphagia, 3:171-178, 1989.

[337] J. Dent, W.J. Dodds, R.H. Friedman, T. Sekiguichi, W.J. Hogan, and R.C. Arndorfer. Mechanisms of lower gastroesophageal reflux in recumbent asymptomatic human subjects. $J$ Clin Invest, 65:256-267, 1980.

[338] W.J. Dodds, E.T. Stewart, and J.A. Logemann. Physiology and radiology of the normal oral and pharyngeal phases of swallowing. Am J Roentgenol, 154:953-963, 1990.

[339] R. Hagen, A. Haase, D. Matthaei, and D. Henrich. Oropharyngeale Funktionsdiagnostik mit der FLASH-MR-Tomographie. Hals Nasen Ohren, 38:421-425, 1990.

[340] R.J. Gilbert, S.B.S. Daftary, P. Woo, S. Seltzer, S.M. Shapshay, and R.M. Weisskoff. Echo-planar magnetic resonance imaging of deglutitive vocal fold closure: Normal and pathologic patterns of displacement. Laryngoscope, 106:568-572, 1996.

[341] Y. Suto, M. Kamba, and T. Kato. Technical note: dynamic analysis of the pharynx during swallowing using turbo-FLASH magnetic resonance imaging combined with an oral positive contrast agent - A preliminary study. Brit J Radiol, 68:1099-1102, 1995.

[342] R.F. Flaherty, S. Seltzer, T. Campbell, R.M. Weisskoff, and R.J. Gilbert. Dynamic magnetic resonance imaging of vocal cord closure during deglutition. Gastroenterol, 109:843-849, 1995.

[343] R.J. Gilbert, S. Daftary, T.A.C., and R.M. Weisskoff. Patterns of lingual tissue deformation associated with bolus containment and propulsion during deglutition as determined by echo-planar MRI. J Magn Reson Imaging, 8:554-560, 1998.

[344] J.M. Foucar, P. Caq0entierl, D. Pajoni, P. Rabischong, and C. Pharaboz. Kinetic magnetic resonance imaging analysis of swallowing: a new approach to pharyngeal function. Surg Radiol Anat, 20:53-55, 1998.

[345] A. Anagnostara, S. Stoeckli, O.M. Weber, and S.S. Kollias. Evaluation of the anatomical and functional properties of deglutition with various kinetic high-speed MRI sequences. J Magn Reson Imaging, 14:194-199, 2001.

[346] D.M. Hartl, M. Albiter, F. Kolb, B. Luboinski, and R. Sigal. Morphologic parameters of normal swallowing events using single-shot fast spin echo dynamic MRI. Dysphagia, 18:255-262, 2003.

[347] D.M. Hartl, F. Kolb, E. Bretagne, P. Marandas, and R. Sigal. Cine magnetic resonance imaging with single-shot fast spin echo for evaluation of dysphagia and aspiration. Dysphagia, pages $156-162,2006$.

[348] R.D. Riordan, M. Khonsari, J. Jeffries, G.F. Maskell, and P.G. Cook. Pineapple juice as a negative oral contrast agent in magnetic resonance cholangiopancreatography: a 
preliminary evaluation. Brit J Radiol, 77:991-999, 2004.

[349] K.S. Nayak, B.A. Hargreaves, B.S. Hu, D.G. Nishimura, J.M. Pauly, and C.H. Meyer. Spiral balanced steady-state free precession cardiac imaging. Magn Reson Med, 53:14681473, 2005.

[350] O.P. Simonetti, J.P. Finn, R.D. White, G. Laub, and D.A. Henry. "black blood" T2weighted inversion-recovery MR imaging of the heart. Radiol, 199:49-57, 1996.

[351] R.R. Edelman, W.J. Manning, D. Burstein, and S. Paulin. Coronary arteries: breathhold MR angiography. Radiol, 181:641-643, 1991.

[352] V.M. Runge, J.A. Clanton, C.L. Partain, and A.E. James. Respiratory gating in magnetic resonance imaging at 0.5 tesla. Radiol, 151:521-523, 1984.

[353] R.L. Ehman, M.T. McNamara, M. Pallack, H. Hricak, and C.B. Higgins. Magnetic resonance imaging with respiratory gating: techniques and advantages. Am J Roentgenol, 143:1175-1182, 1984.

[354] T.A. Spraggins. Wireless retrospective gating: application to cine cardiac imaging. Magn Reson Imaging, 8:675-681, 1990.

[355] A.C. Larson, R.D. White, G. Laub, E.R. McVeigh, D. Li, and O.P. Simonetti. Selfgated cardiac cine MRI. Magn Reson Med, 51:93-102, 2004.

[356] E. Heijman, W. de Graaf, P. Niessen, A. Nauerth, G. van Eys, L. de Graaf, K. Nicolay, and G.J. Strijkers. Comparison between prospective and retrospective triggering for mouse cardiac MRI. NMR Biomed, 20:439-447, 2007.

[357] M. Buehrer, J. Curcic, P. Boesiger, and S. Kozerke. Prospective self-gating for simultaneous compensation of cardiac and respiratory motion. Magn Reson Med, 60:683-690, 2008.

[358] M. Uecker. Nonlinear Reconstruction Methods for Parallel Magnetic Resonance Imaging. PhD thesis, University of Goettingen, 2009.

[359] C.L. Dumoulin, S.P. Souza, and R.D. Darrow. Real-time position monitoring of invasive devices using magnetic resonance. Magn Reson Med, 29:411-415, 1993.

[360] S.R. Yutzy and J.L. Duerk. Pulse sequences and system interfaces for interventional and real-time MRI. J Magn Reson Imaging, 27:267-275, 2008.

[361] R.J. Kim, D.S. Fieno, T.b. Parrish, K. Harris, E.-L. Chen, O. Simonetti, J. Bundy, J.Paul. Frahm J. Klocke, and R.M. Judd. Relationship of MRI delayed contrast enhancement to irreversible injury, infarct age, and contractile function. Circulation, 100:1992-2002, 1999.

[362] J.M. Wild, M.N.J. Paley, L. Kasuboski, A. Swift, S. Fichele, N. Woodhouse, P.D. Griffiths, and E.J.R. van Beek. Dynamic radial projection MRI of inhaled hyperpolarized 3He gas. Magn Reson Med, 49:991-997, 2003.

[363] J. Brossmann, L.R. Frank, J.M. Pauby, R.D. Boutin, R.A. Pedowitz, P. Haghighi, and D. Resnick. Short echo time projection reconstruction MR imaging of cartilage: Comparison with fat-suppressed spoiled grass and magnetization transfer contrast mr imaging. Radiol, 203:501-507, 1997.

[364] C. P. Slichter. Principles of Magnetic Resonance. Springer, 1996. 
[365] C.E. Shannon. Communication in the presence of noise, volume 37. Proc Institute of Radio Engineers, 1949.

[366] H. Nyquist. Certain topics in telegraph transmission theory, volume 47. Trans AIEE, 1928. 


\section{Abbreviations}

\begin{tabular}{ll} 
ADC & analog-to-digital converter \\
$B_{0}$ & main magnetic field \\
$B W$ & bandwidth \\
CNR & contrast-to-noise ratio \\
CSF & cerebrospinal fluid \\
CS & compressed sensing \\
CT & computed tomography \\
DCF & density compensation function \\
DCE-MRI & dynamic contrast enhanced MRI \\
DFT & discrete Fourier transformation \\
ECG & electrocardiography \\
EPI & echo planar imaging \\
ETL & echo train length \\
$F A$ & flip angle ( $\alpha$ ) \\
$f a_{K}$ & keyhole factor \\
$f a_{S}$ & sparse factor \\
FBP & filtered back-projection \\
FFT & fast Fourier transformation \\
FID & free induction decay \\
FISP & fast imaging with steady-state precession \\
FLASH & fast low angle shot \\
fMRI & functional MRI \\
FOV & field of view \\
fps & frames per second \\
FSE & fast spin echo \\
Gd-DTPA & gadolinium-diethylenetriaminepentaacetic acid \\
GRASE & gradient- and spin-echo \\
GRASS & gradient-recalled echo in the steady state \\
GRE & gradient echo \\
\hline
\end{tabular}


HASTE half-Fourier acquisition single-shot turbo spin-echo

HYPR highly constrained back-projection

I spin quantum number

IR inversion recovery

KWIC $\quad k$-space weighted image contrast

$L \quad$ angular momentum

MRI magnetic resonance imaging

M magnetization

$M_{z} \quad$ longitudinal magnetization

$M_{x y} \quad$ transversal magnetization

NMR nuclear magnetic resonance

$N_{s} \quad$ number of samples

$N_{A V E} \quad$ number of signal/acquisition averages

$N_{P E} \quad$ number of phase encoding steps

$N_{\text {seg }} \quad$ number of (radial) segments/interleaves

$N_{V} \quad$ number of views

PAT parallel acquisition technique / parallel imaging technique / parallel imaging

PCA principal component analysis

PD proton density $(\rho)$

$\mathrm{PF} \quad$ partial Fourier

PNS peripheral nerve stimulation

ppm parts per million

PR projection reconstruction

PSF point spread function

RARE rapid acquisition with relaxation enhancement

RF radio frequency

ROI region of interest

SAR specific absorption rate

SD standard deviation

SE $\quad$ spin echo

SENSE sensitivity encoding

SLT slice thickness

SNR signal-to-noise ratio

SSFP steady state free precession 
STE stimulated echo

STEAM stimulated echo acquisition mode

T1 spin-lattice relaxation time

T2 spin-spin relaxation time

T2* effective spin-spin relaxation time

TA acquisition time

TE echo time

$T E_{\text {eff }} \quad$ effective echo time

TI inversion time

TM middle time

TMJ temporomandibular joint

$T R \quad$ repetition time

TU update time

TureFISP true fast imaging with steady state precession

UNFOLD unaliasing by Fourier-encoding the overlaps using the temporal dimension

$\Delta k \quad$ distance between $k$-space data samples

$\Delta t \quad$ dwell time

$\Delta \varnothing \quad$ angular distance between $k$-space (radial) views

$\Delta \emptyset_{I N C} \quad$ incremental angle between $k$-space (radial) views

$\omega_{0} \quad$ Larmor frequency

$\hbar \quad$ Planck's constant

$\gamma \quad$ gyromagnetic ratio

$\mu \quad$ magnetic moment

$\delta \quad$ chemical shift 


\section{Acknowledgement}

I consider myself very fortunate to have the opportunity to accomplish my doctoral thesis at Biomedizinische NMR Forschungs GmbH at Max-Planck-Institute for Biophysical Chemistry. It is such an honor to have worked with so many talented scientists in this excellent research environment. Therefore, I am very grateful to, first and forever, Prof. Dr. Jens Frahm for providing me such a chance and for his continuous support of my work.

I would also like to give my gratitude to Prof. Dr. Ulrich Parlitz and Prof. Dr. Annette Zippelius for their supervision and interest in this work, and I thank Dr. Klaus-Dietmar Merboldt for offering his invaluable instructions and suggestions from the very beginning of my work.

I am especially grateful to Dr. Dirk Voit and Dr. Kai Tobias Block for their technical support, and I thank all members of Biomedizinische NMR Forschungs $\mathrm{GmbH}$ for creating a pleasant working atmosphere and for providing help during my thesis.

I would like to express my appreciation to all my friends for encouraging and supporting me during my studies. Many of them made contributions to this work as volunteers in the experiments. Being with them for these three years in this cozy city is a great pleasure for me.

Special thanks to Dr. Sabine Hofer and Yuan-Ming Chiao for assistance in improving the quality of this thesis.

On a personal note, I am much indebted to my parents and my wife. I thank you for the constant support, encouragement, and your love, which inspire my efforts in every possible way. I am who I am because of you. 


\title{
Curriculum Vitae
}

\author{
Personal Information \\ Name ZHANG, Shuo \\ Date of Birth May 17, 1981 \\ Place of Birth Shanghai, China \\ Gender Male \\ Nationality Chinese
}

\section{Academic Background}

$1999-2003$

Main Area of Study

Graduation Thesis

Publication

$2003-2005$

Main Area of Study

Graduation Thesis

Publication

2006 - Present

PhD Program

Main Area of Study

Graduation Thesis

Publication
Bachelor of Engineering (B. Eng.) in Biomedical Engineering

Shanghai Jiao Tong University /

Shanghai Second Medical University, China

Medical instrumentation and technology, bioinformatics

"Application of XML in Electronic Medical Record"

S. Zhang and Z.H. Yao. Application of XML in Electronic Medical Records.

China Contemporary Medicine 2003(68); 9(5): 45-47

Master of Engineering (M. Eng.) in Biomedical Engineering

Martin-Luther-Universität Halle-Wittenberg /

Hochschule Anhalt (FH), Germany

Medical physics in diagnostic radiology, biophysical technology

"Proton MR Spectroscopy and Diffusion Tensor MR Imaging: Establishment of Two New Radiodiagnostic Methods for Clinical Applications"

M. Knörgen, S. Zhang, and R.P. Spielmann. Ortsaufgelöste MR-Spektroskopie: Auswerte-methoden im Vergleich. 87th Deutscher Röntgenkongress 2006. 24-27 May 2006, Berlin, Germany (Conference Poster)

S. Zhang. The Primary Establishment and Implementation of Diffusion Tensor Imaging for Clinical Routine Applications. Chinese Biomedical Engineering 2007; 26(5): 796-800

Doctor of Philosophy (Ph.D.)

Biomedizinische NMR Forschungs $\mathrm{GmbH}$ am Max-Planck-Institut für biophysikalishce Chemie / Georg-August-University Göttingen, Germany

Theoretical and Computational Neuroscience (PTCN)

MR Physics, MRI development and application

"Real-time Magnetic Resonance Imaging"

S. Zhang, K.T. Block, and J. Frahm. Magnetic Resonance Imaging in Real Time - Advances Using Radial FLASH. Journal of Magnetic Resonance Imaging, accepted, 2009 
